\title{
Collection And Characterization Of Particulate From The Tore Supra Tokamak (December 1999 Vent)
}

J. Phillip Sharpe

December 2000

Idaho National Engineering and Environmental Laboratory Bechtel BWXT Idaho, LLC 
INEEL/EXT-2000-01619

December 2000

\title{
Collection and Characterization of Particulate from the Tore Supra Tokamak (December 1999 Vent)
}

\author{
J. Phillip Sharpe
}

December 2000

\author{
Fusion Safety Program \\ Nuclear Engineering Development and Research Department \\ Idaho National Engineering and Environmental Laboratory \\ BWXT Idaho \\ Idaho Falls, ID 83415
}

Prepared for the Office of Fusion Energy Sciences

Office of Science U.S. Department of Energy Under DOE Idaho Operations Office Contract DE-AC07-99ID13727 


\section{Table of Contents}

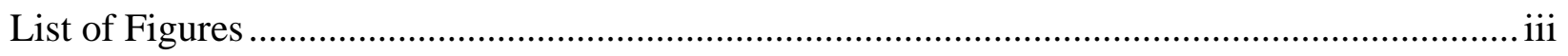

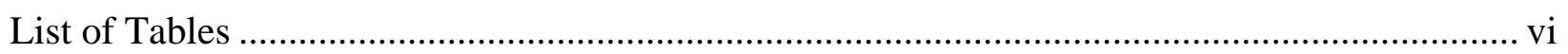

List of Acronyms ………………….............................................................................. vii

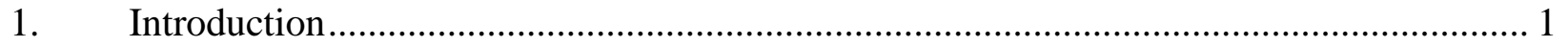

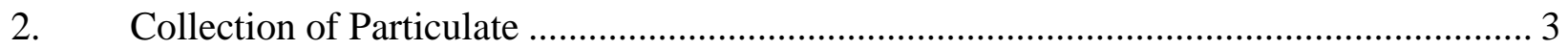

2.1. Methods of Collection....................................................................................... 3

2.2. Collection Locations in Tore Supra ………………............................................... 4

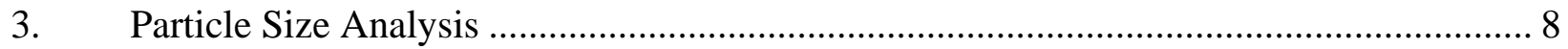

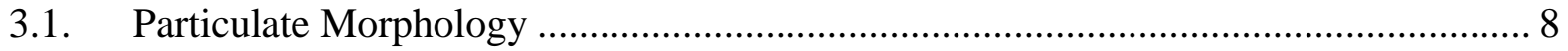

3.2. Size Analysis by Optical Microscopy ……….................................................... 9

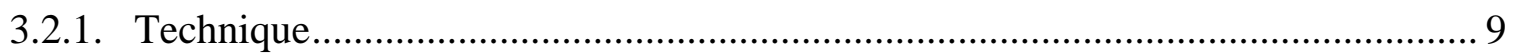

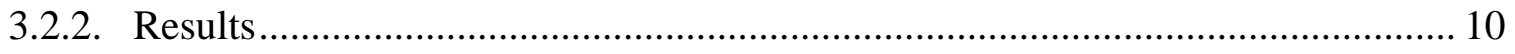

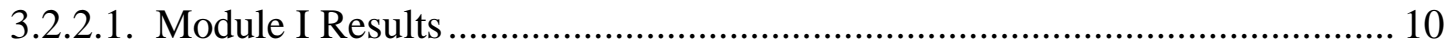

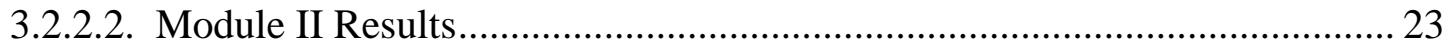

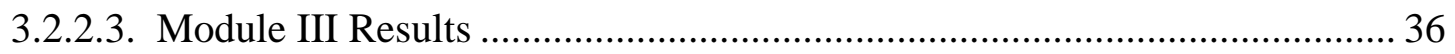

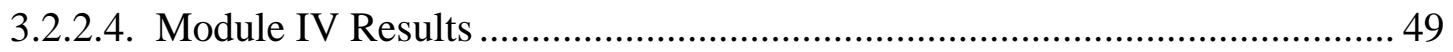

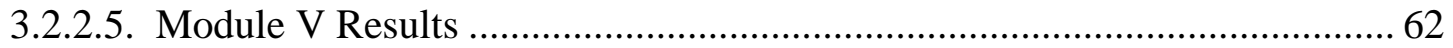

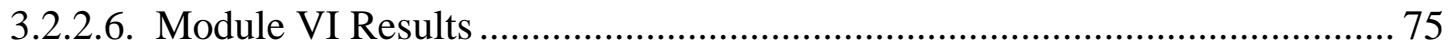

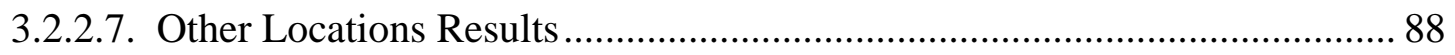

3.3. Size Analysis with the Coulter LS130 Particle Analysis System ............................... 96

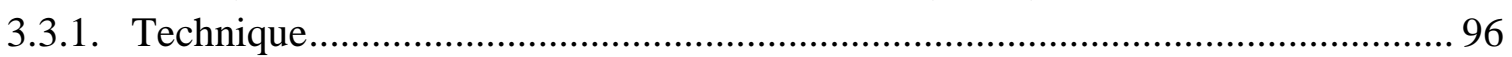

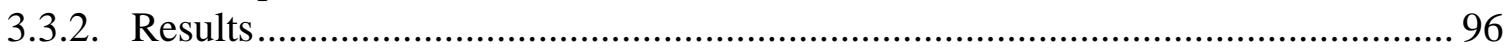

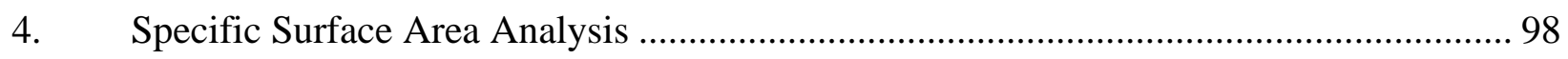

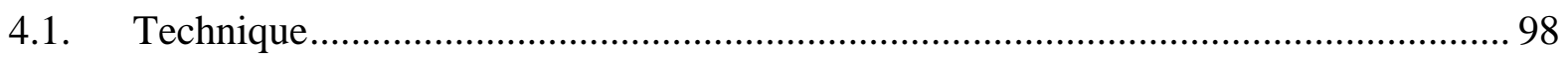

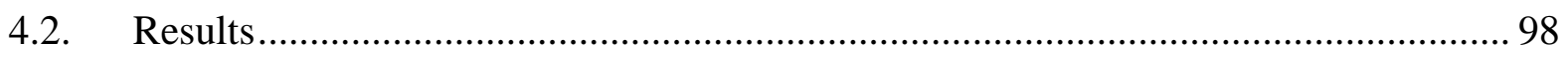

5. Particulate Composition Analysis by SEM/EDX Analysis ............................................ 104

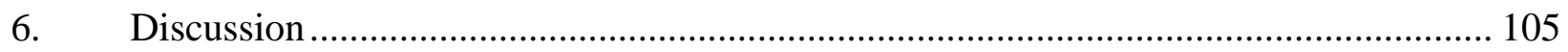

6.1. Distribution of Dust Surface Mass Density ………................................................. 105

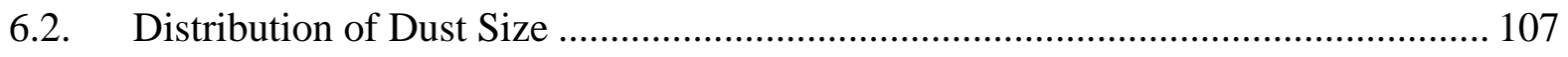

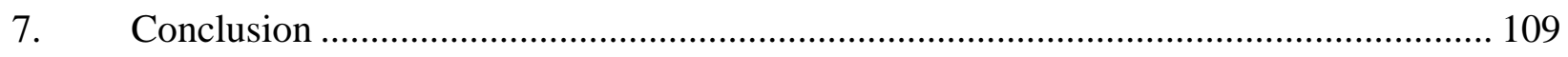

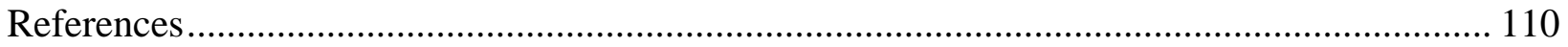




\section{List of Figures}

Figure 1.1 View of the Tore Supra plasma chamber with various components identified........... 2

Figure 2.1 Schematic diagram of the filtered vacuum particle collection technique.................... 3

Figure 2.2 Poloidal sampling locations 1 and 2. Location 1 is on the outboard plasma chamber

wall, and location 2 is the outboard vessel wall along the centerline groove...................... 5

Figure 2.3 Poloidal sampling location 3- around outboard observation port. ............................ 5

Figure 2.4 Poloidal sampling location 4- surface of divertor neutralizer. ................................... 5

Figure 2.5 Poloidal sampling locations 5 and 6 . Location 5 is on and between the port ripple

protection, and location 6 is around and inside the port. .......................................... 5

Figure 2.6 Poloidal sampling locations 7 and 8. Location 7 is a sweep along the entire column height, and location 8 is a short, midplane sweep................................................... 6

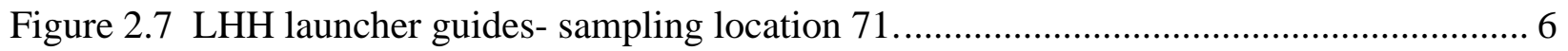

Figure 2.8 Poloidal sampling locations 9, 10, 11, and 12 ................................................... 6

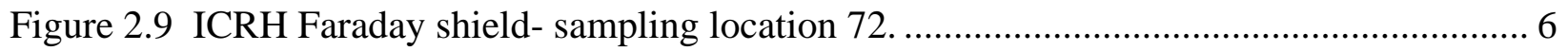

Figure 2.10 ECRH antenna mirrors- sampling location 73 ............................................... 7

Figure 2.11 Detail of horizontal pump limiter ionic side- sampling locations 74 (throat) and 76 (face). Sampling locations 75 and 77 are the corresponding electronic positions on the

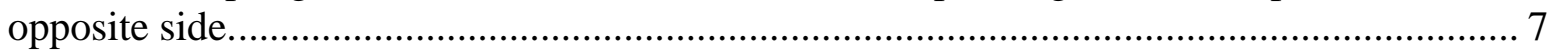

Figure 2.12 Horizontal pump limiter. In this view, the right side of the limiter is the ionic face, and the left side is the electronic face. ................................................................ 7

Figure 3.1 Representative SEM photomicrographs demonstrating various particle morphologies observed in dust samples from Tore Supra.................................................................... 8

Figure 3.2 TEM photomicrographs of dust collected with replicating tape. ............................. 9

Figure 3.3 Module I, Sample 1 (TSFH22-01) count-based particle size distribution................. 11

Figure 3.4 Module I, Sample 2 (TSFH01-01) count-based particle size distribution.................. 12

Figure 3.5 Module I, Sample 3 (TSFH23-01) count-based particle size distribution................. 13

Figure 3.6 Module I, Sample 4 (TSFH24-01) count-based particle size distribution.................. 14

Figure 3.7 Module I, Sample 5 (TSFH25-01) count-based particle size distribution................. 15

Figure 3.8 Module I, Sample 6 (TSFH05-01) count-based particle size distribution................. 16

Figure 3.9 Module I, Sample 7 (TSFH07-01) count-based particle size distribution................. 17

Figure 3.10 Module I, Sample 8 (TSFH06-01) count-based particle size distribution............... 18

Figure 3.11 Module I, Sample 9 (TSFH04-01) count-based particle size distribution............... 19

Figure 3.12 Module I, Sample 10 (TSFH02-01) count-based particle size distribution............. 20

Figure 3.13 Module I, Sample 10A (TSFH21-01) count-based particle size distribution.......... 21

Figure 3.14 Module I, Sample 10B (TSFH03-01) count-based particle size distribution.......... 22

Figure 3.15 Module II, Sample 11 (TSFH09-01) count-based particle size distribution........... 24

Figure 3.16 Module II, Sample 12 (TSFH18-01) count-based particle size distribution........... 25

Figure 3.17 Module II, Sample 13 (TSFH08-01) count-based particle size distribution........... 26

Figure 3.18 Module II, Sample 14 (TSFH19-01) count-based particle size distribution............ 27

Figure 3.19 Module II, Sample 15 (TSFH17-01) count-based particle size distribution........... 28

Figure 3.20 Module II, Sample 16 (TSFH14-01) count-based particle size distribution. ........... 29 
Figure 3.21 Module II, Sample 17 (TSFH27-03) count-based particle size distribution........... 30

Figure 3.22 Module II, Sample 18 (TSFH11-01) count-based particle size distribution........... 31

Figure 3.23 Module II, Sample 19 (TSFH10-01) count-based particle size distribution............ 32

Figure 3.24 Module II, Sample 20 (TSFH13-01) count-based particle size distribution........... 33

Figure 3.25 Module II, Sample 20A (TSFH15-01) count-based particle size distribution. ........ 34

Figure 3.26 Module II, Sample 20B (TSFH16-01) count-based particle size distribution......... 35

Figure 3.27 Module III, Sample 21 (TSFH05-02) count-based particle size distribution........... 37

Figure 3.28 Module III, Sample 22 (TSFH23-02) count-based particle size distribution.......... 38

Figure 3.29 Module III, Sample 23 (TSFH06-02) count-based particle size distribution.......... 39

Figure 3.30 Module III, Sample 24 (TSFH11-02) count-based particle size distribution.......... 40

Figure 3.31 Module III, Sample 25 (TSFH08-02) count-based particle size distribution.......... 41

Figure 3.32 Module III, Sample 26 (TSFH10-02) count-based particle size distribution........... 42

Figure 3.33 Module III, Sample 27 (TSFH09-02) count-based particle size distribution.......... 43

Figure 3.34 Module III, Sample 28 (TSFH07-02) count-based particle size distribution.......... 44

Figure 3.35 Module III, Sample 29 (TSFH22-02) count-based particle size distribution.......... 45

Figure 3.36 Module III, Sample 30 (TSFH25-02) count-based particle size distribution.......... 46

Figure 3.37 Module III, Sample 30A (TSFH24-02) count-based particle size distribution........ 47

Figure 3.38 Module III, Sample 30B (TSFH21-02) count-based particle size distribution. ....... 48

Figure 3.39 Module IV, Sample 31 (TSFH18-02) count-based particle size distribution........... 50

Figure 3.40 Module IV, Sample 32 (TSFH16-02) count-based particle size distribution.......... 51

Figure 3.41 Module IV, Sample 33 (TSFH01-03) count-based particle size distribution.......... 52

Figure 3.42 Module IV, Sample 34 (TSFH14-02) count-based particle size distribution.......... 53

Figure 3.43 Module IV, Sample 35 (TSFH13-02) count-based particle size distribution.......... 54

Figure 3.44 Module IV, Sample 36 (TSFH02-03) count-based particle size distribution.......... 55

Figure 3.45 Module IV, Sample 37 (TSFH04-03) count-based particle size distribution........... 56

Figure 3.46 Module IV, Sample 38 (TSFH12-02) count-based particle size distribution.......... 57

Figure 3.47 Module IV, Sample 39 (TSFH03-03) count-based particle size distribution........... 58

Figure 3.48 Module IV, Sample 40 (TSFH17-02) count-based particle size distribution.......... 59

Figure 3.49 Module IV, Sample 40A (TSFH19-02) count-based particle size distribution........ 60

Figure 3.50 Module IV, Sample 40B (TSFH15-02) count-based particle size distribution........ 61

Figure 3.51 Module V, Sample 41 (TSFH08-03) count-based particle size distribution........... 63

Figure 3.52 Module V, Sample 42 (TSFH24-03) count-based particle size distribution........... 64

Figure 3.53 Module V, Sample 43 (TSFH11-03) count-based particle size distribution............ 65

Figure 3.54 Module V, Sample 44 (TSFH21-03) count-based particle size distribution........... 66

Figure 3.55 Module V, Sample 45 (TSFH09-03) count-based particle size distribution........... 67

Figure 3.56 Module V, Sample 46 (TSFH07-03) count-based particle size distribution........... 68

Figure 3.57 Module V, Sample 47 (TSFH23-03) count-based particle size distribution........... 69

Figure 3.58 Module V, Sample 48 (TSFH06-03) count-based particle size distribution........... 70

Figure 3.59 Module V, Sample 49 (TSFH05-03) count-based particle size distribution........... 71

Figure 3.60 Module V, Sample 50 (TSFH10-03) count-based particle size distribution........... 72 
Figure 3.61 Module V, Sample 50A (TSFH22-03) count-based particle size distribution........ 73

Figure 3.62 Module V, Sample 50B (TSFH25-03) count-based particle size distribution. ........ 74

Figure 3.63 Module IV, Sample 51 (TSFH12-03) count-based particle size distribution.......... 76

Figure 3.64 Module IV, Sample 52 (TSFH17-03) count-based particle size distribution.......... 77

Figure 3.65 Module IV, Sample 53 (TSFH13-03) count-based particle size distribution.......... 78

Figure 3.66 Module IV, Sample 54 (TSFH27-02) count-based particle size distribution.......... 79

Figure 3.67 Module IV, Sample 55 (TSFH26-03) count-based particle size distribution........... 80

Figure 3.68 Module IV, Sample 56 (TSFH27-01) count-based particle size distribution........... 81

Figure 3.69 Module IV, Sample 57 (TSFH14-03) count-based particle size distribution.......... 82

Figure 3.70 Module IV, Sample 58 (TSFH26-02) count-based particle size distribution.......... 83

Figure 3.71 Module IV, Sample 59 (TSFH19-03) count-based particle size distribution.......... 84

Figure 3.72 Module IV, Sample 60 (TSFH15-03) count-based particle size distribution.......... 85

Figure 3.73 Module IV, Sample 60A (TSFH16-03) count-based particle size distribution........ 86

Figure 3.74 Module IV, Sample 60B (TSFH18-03) count-based particle size distribution........ 87

Figure 3.75 Sample 71 (TSFH20-02) count-based particle size distribution. ........................... 89

Figure 3.76 Sample 72 (TSFH20-01) count-based particle size distribution. .......................... 90

Figure 3.77 Sample 73 (TSFH26-01) count-based particle size distribution. .......................... 91

Figure 3.78 Sample 74 (TSFH04-02) count-based particle size distribution. .......................... 92

Figure 3.79 Sample 75 (TSFH01-02) count-based particle size distribution. .......................... 93

Figure 3.80 Sample 76 (TSFH02-02) count-based particle size distribution. ........................... 94

Figure 3.81 Sample 77 (TSFH03-02) count-based particle size distribution. .......................... 95

Figure 3.82 Volume-based size distribution of Module V, Sample 50A (TSFH22-03)............ 97

Figure 4.1 Adsorbed Volume (a) and adsorption isotherm (b) for (iv) TSFH21-02, Sample 30B.

Figure 4.2 Adsorbed Volume (a) and adsorption isotherm (b) for (iii) TSFH16-02, Sample 32. 101

Figure 4.3 Adsorbed Volume (a) and adsorption isotherm (b) for (i) TSFH15-02,

Sample 40B. 102

Figure 4.4 Adsorbed Volume (a) and adsorption isotherm (b) for (ii) TSFH19-02,

Sample 40A 103

Figure 5.1 SEM images of (a) smaller dust collected from Location 6 and (b) larger debris from

Location 12 of Module I. 104

Figure 5.2 Particles from Location 6, of Module I (Sample 5, TSFH25-01). ........................ 104

Figure 6.1 Distribution of dust surface mass density in Tore Supra.................................... 106

Figure 6.2 Distribution of dust size in Tore Supra.......................................................... 108 


\section{List of Tables}

Table 2.1 Sampling locations for dust collection in Tore Supra............................................ 4

Table 3.1 Summary of dust collected from locations in Tore Supra Module I. ........................ 10

Table 3.2 Summary of dust collected from locations in Tore Supra Module II....................... 23

Table 3.3 Summary of dust collected from locations in Tore Supra Module III....................... 36

Table 3.4 Summary of dust collected from locations in Tore Supra Module IV ...................... 49

Table 3.5 Summary of dust collected from locations in Tore Supra Module V........................ 62

Table 3.6 Summary of dust collected from locations in Tore Supra Module VI...................... 75

Table 3.7 Summary of dust collected from specific singular locations.................................. 88

Table 4.1 Summary of results from BET SSA measurements............................................... 99 


\section{List of Acronyms}

\begin{tabular}{|c|c|}
\hline BET .... & Brunauer, Emmett, Teller \\
\hline CFC.. & Carbon Fiber Composite \\
\hline CMD .. & .Count Median Diameter \\
\hline EDX ... & Energy-Dispersive X-ray \\
\hline GMD .. & Geometric Mean Diameter \\
\hline GSD.... & ..Geometric Standard Deviation \\
\hline INEEL & $\begin{array}{l}\text {..Idaho National Engineering and Environmental } \\
\text { Laboratory }\end{array}$ \\
\hline SEM ... & .Scanning Electron Microscopy \\
\hline$S S A \ldots .$. & Specific Surface Area \\
\hline TEM ... & ..Tunneling Electron Microscopy \\
\hline
\end{tabular}




\section{Collection and Characterization of Particulate from the Tore Supra Tokamak (December 1999 Vent)}

\section{Introduction}

Particulate generated during the operation of a fusion device contributes to the radiological source term associated with accident scenarios in the device. ${ }^{1,2}$ Understanding the mechanisms generating the particulate and correctly describing its physical and chemical behavior is essential for safety analyses of future fusion devices. Knowledge of these mechanisms is gained by collecting and characterizing particulate matter from operating fusion facilities.

Tokamak dust, the particulate matter generated during the operation of a tokamak fusion device, was collected from Tore Supra in December 1999, during the initial phase of the scheduled shutdown for installation of advanced plasma facing components. Tore Supra, located at CEA Cadarache, France, is presently the third largest operating tokamak with the capability of long-pulse operation. Eighteen super-conducting coils produce the $4.5 \mathrm{~T}$ magnetic field inside a vessel $2.4 \mathrm{~m}$ in major radius and $1.2 \mathrm{~m}$ in minor radius. Limiter and divertor regimes of operation are possible. In the divertor regime, the circular magnetic configuration is ergodized by six outboard resonant divertor modules that are covered with $12 \mathrm{~m}^{2}$ of carbon fiber composite (CFC) tiles. In addition, some field lines are diverted to actively cooled neutralizing plates made of $\mathrm{CuCrZr}$ bars covered with $\mathrm{B}_{4} \mathrm{C}$. In the limiter regime, the plasma leans on the actively cooled inboard first wall or on a set of inertially cooled pumped limiters. The first wall area of $12 \mathrm{~m}^{2}$ is covered with both polycrystalline graphite tiles (83\%) and CFC tiles (17\%). The single outboard limiter is constructed of pyrolitic graphite, and the four toroidally symmetric bottom limiters are constructed of CFC. Figure 1.1 displays the relative location of plasma facing components within the plasma chamber of Tore Supra.

In this report, we present in Section 2 the methods used to collect and analyze this dust and describe the selection of sampling locations. Section 3 includes a discussion of observed particle morphology, results from optical microscopy particle size analysis, and results of particle size measurements performed with a laser diffraction analysis system. The results from specific surface area analyses are given in Section 4, and particle composition analyses by SEM/EDX are discussed in Section 5. A discussion of the results and the implications of the Tore Supra dust are given in Section 6. Conclusions of this current work are summarized in Section 7. 


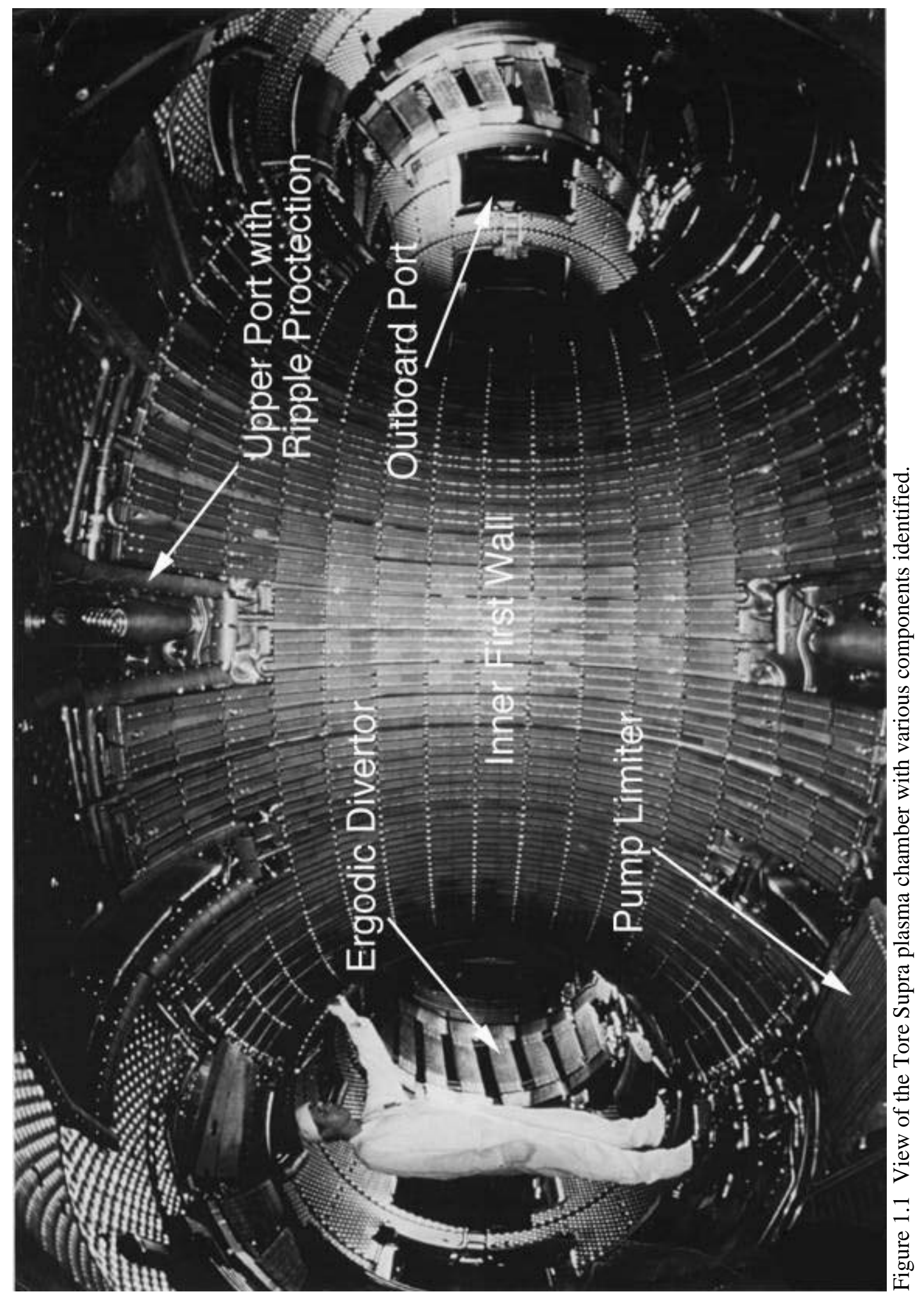




\section{Collection of Particulate}

Part 1 of this section describes the techniques used to sample dust from inner surfaces of the Tore Supra vacuum vessel and the initial preparation of these samples for analysis. In part 2, details of the sampling locations are given.

\subsection{Methods of Collection}

The primary method for collecting particulate from various locations in the Tore Supra vacuum vessel was the filtered vacuum technique used extensively in previous dust collection campaigns of other fusion devices. ${ }^{3}$ Figure 2.1 demonstrates this simple and reliable technique. Particulate collection is achieved with the use of a small vacuum pump connected to a $50 \mathrm{~mm}$ filter housing. A filter of $0.02 \mu \mathrm{m}$ pore size is placed within the housing, and a 0.25 in diameter probe tip is connected for sampling. Various probe lengths are available for surface or deep recess sampling. The volumetric flow rate of the vacuum filter system is 15 standard liters per minute (slpm), sufficient to entrain loosely bound particles into the flow stream as the probe tip is moved over the sample surface. Samples are acquired by placing the probe tip approximately 5 $\mathrm{mm}$ above the surface and sweeping with a velocity of $5 \mathrm{~cm} / \mathrm{s}$. The particles are deposited on the filter substrate, which is later removed for analysis. Upon disassembly in the laboratory, the filter substrate and any particle material not adherent to the substrate are removed from the filter housing, weighed for collected mass, and placed in metal storage tins. The filtered vacuum method has an estimated collection limit of $0.1 \mu \mathrm{m}$ diameter for individual particles, as indicated by the absence of smaller particles when a filter substrate is observed via electron microscopy. Particles of size less than $0.1 \mu \mathrm{m}$ have been observed only as a component to larger agglomerates.

Two additional collection techniques were used in this campaign. Swipe tests were taken at prescribed locations within the vessel using quartz fiber filter paper. Metallurgical replicating tape (acetate tape) was also used. These techniques allow collection of particulate with very small sizes, possibly demonstrating the existence of particulate at sizes beyond the collection

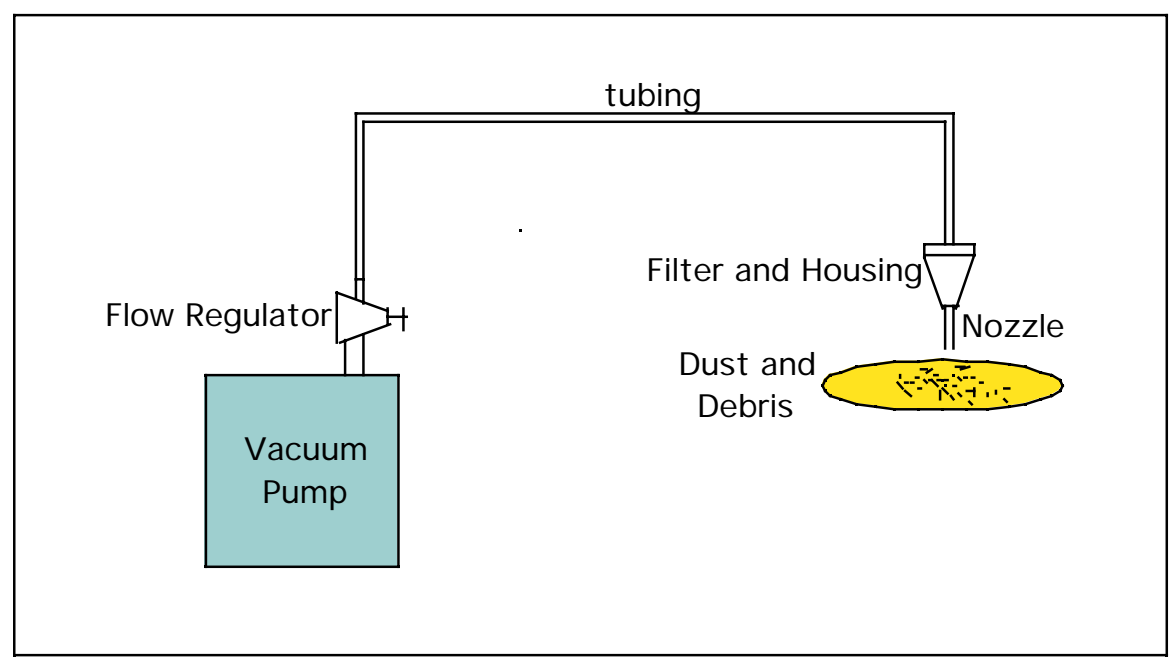

Figure 2.1 Schematic diagram of the filtered vacuum particle collection technique. 
capability of the vacuum filter technique. Detailed analyses of samples obtained from swipe tests and replicating tape are, however, difficult due to the non-uniform structure of the collection substrate. Hence these methods of collection were used to investigate particle morphology (with SEM and TEM) and to examine particle size in a qualitative fashion.

\subsection{Collection Locations in Tore Supra}

Locations for dust sampling in the Tore Supra vessel were selected based on the objective of identifying position-dependent trends in particulate deposition. Collection was performed at 12 poloidal locations in each of the six toroidal modules that compose the vessel. Table 2.1 provides the details for each sampling location. Samples were also obtained at locations of certain structures exposed to plasma, specifically the horizontal pumped limiter, the electron cyclotron resonance heating antenna mirrors, the ion cyclotron resonance heating Faraday shield, and the lower hybrid heating launcher guides. Values for the sampled area of each location were determined from area measurements during collection in the first module. Corresponding poloidal locations in subsequent modules were sampled in a similar fashion such that the sampled areas are equivalent in each module.

Table 2.1 Sampling locations for dust collection in Tore Supra.

\begin{tabular}{|c|c|c|c|c|c|}
\hline $\begin{array}{l}\text { Location } \\
\text { Number }\end{array}$ & \multicolumn{2}{|c|}{$\begin{array}{l}\text { Poloidal } \\
\text { angle, } \phi\end{array}$} & $\begin{array}{l}\text { Toroidal } \\
\text { angle*, } \theta\end{array}$ & $\begin{array}{l}\text { Sample Area } \\
\left(\mathrm{cm}^{2}\right)\end{array}$ & Description \\
\hline \multicolumn{6}{|c|}{ Poloidal Locations- Sampled in each of the six modules. } \\
\hline 1 & 0 & \multirow{4}{*}{$\begin{array}{l}\text { outboard } \\
\text { positions }\end{array}$} & 30 & 621.2 & outboard chamber wall, short sweep $\left( \pm 15^{\circ}\right)$ \\
\hline 2 & 0 & & 30 & 1,533 & outboard vessel wall, long sweep $\left( \pm 90^{\circ}\right)$ \\
\hline 3 & 0 & & 40 & 1,800 & on ledge and around outboard observation port \\
\hline 4 & 0 & & 0 & 689.0 & on $\mathrm{B}_{4} \mathrm{C}$ surface of divertor neutralizer \\
\hline 5 & 90 & \multirow{2}{*}{$\begin{array}{l}\text { upper } \\
\text { positions }\end{array}$} & 20 & 1,200 & on and between upper port ripple protection graphite tiles \\
\hline 6 & 90 & & 20 & 658.0 & around upper observation port \\
\hline 7 & 180 & \multirow{2}{*}{$\begin{array}{l}\text { inboard } \\
\text { positions }\end{array}$} & 27 & 428.0 & on inboard first wall protective tiles, long sweep $\left( \pm 90^{\circ}\right)$ \\
\hline 8 & 180 & & 33 & 84.0 & on inboard first wall protective tiles, short sweep $\left( \pm 15^{\circ}\right)$ \\
\hline 9 & 270 & \multirow{4}{*}{$\begin{array}{l}\text { lower } \\
\text { positions }\end{array}$} & 20 & 294.0 & on stainless steel wing on inner first wall \\
\hline 10 & 270 & & 0 & 1,429 & on lower electron collector protective tile surface \\
\hline 11 & 270 & & 20 & 160.0 & on lower vessel surface, left of module center \\
\hline 12 & 270 & & 40 & 480.0 & on lower vessel surface, right of module center \\
\hline \multicolumn{6}{|c|}{ Other Locations. } \\
\hline 71 & \multirow{7}{*}{\multicolumn{2}{|c|}{$\begin{array}{l}\text { all outboard } \\
\text { positions }\end{array}$}} & Module 1 & 2,688 & on lower hybrid heating launcher guides \\
\hline 72 & & & Module 1 & 2,640 & on ion cyclotron resonance heating Faraday shield \\
\hline 73 & & & Module 3 & 5,520 & electron cyclotron resonance heating antenna mirrors \\
\hline 74 & & & Module 2 & 1,500 & on horizontal pump limiter- ionic front face \\
\hline 75 & & & Module 2 & 1,500 & on horizontal pump limiter- electronic front face \\
\hline 76 & & & Module 2 & 200.0 & on horizontal pump limiter- ionic throat \\
\hline 77 & & & Module 2 & 150.0 & on horizontal pump limiter- electronic throat \\
\hline
\end{tabular}

*- measured from the beginning position of each module, so that overall toroidal location of a sample position is determined by: (module number $*\left(60^{\circ}\right)+$ toroidal position angle indicated in the table.

Figure 2.2 through Figure 2.12 display detailed structures of the vessel chamber from which particulate samples were collected via the filtered vacuum technique. Numbered locations in the images correspond to the various poloidal sampling locations given in Table 2.1. 


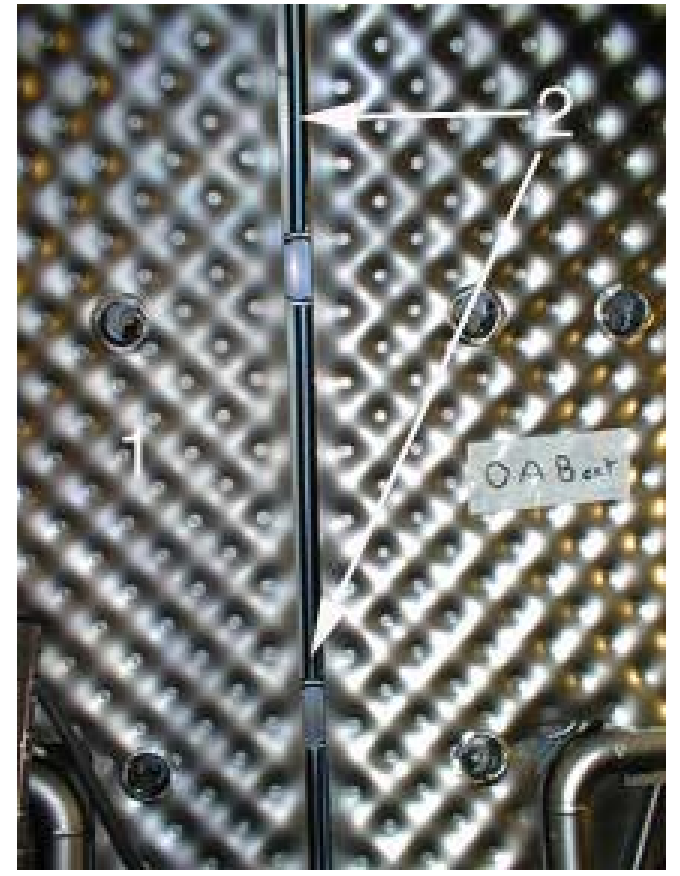

Figure 2.2 Poloidal sampling locations 1 and 2. Location 1 is on the outboard plasma chamber wall, and location 2 is the outboard vessel wall along the centerline groove.

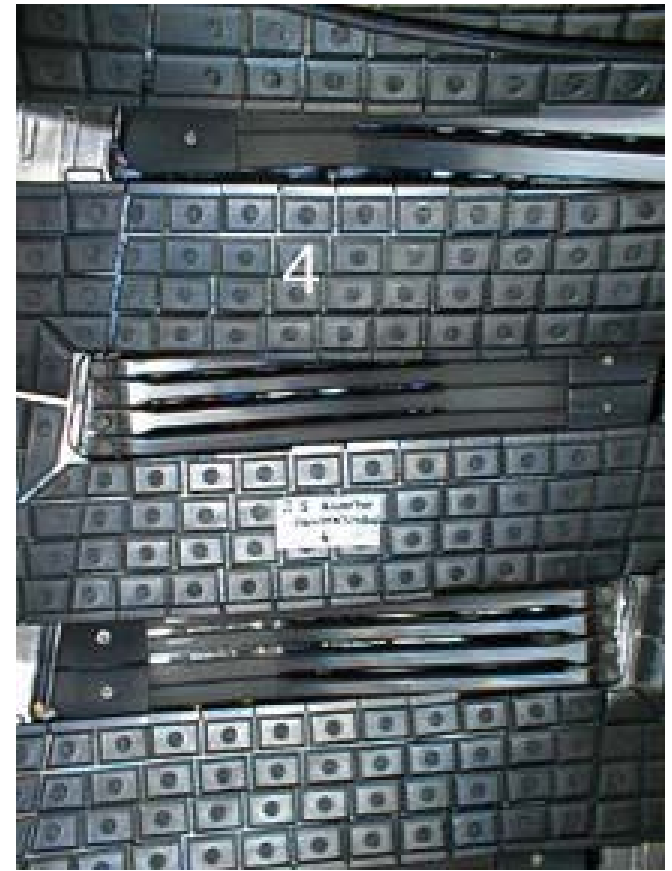

Figure 2.4 Poloidal sampling location 4surface of divertor neutralizer.

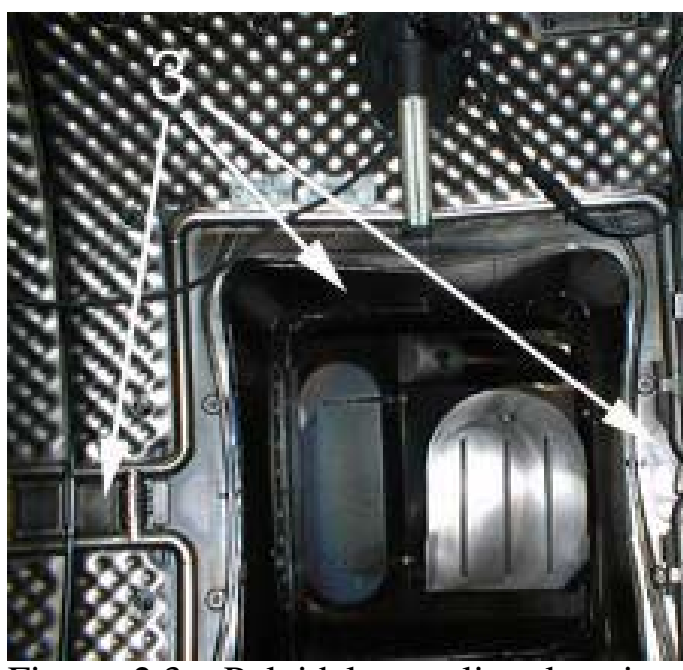

Figure 2.3 Poloidal sampling location 3around outboard observation port.

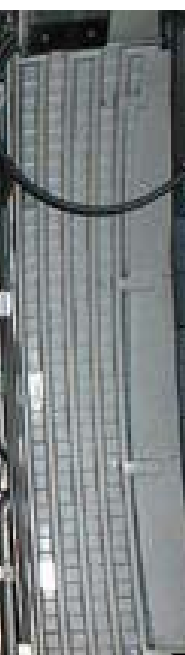

Figure 2.5 Poloidal sampling locations 5 and 6 . Location 5 is on and between the port ripple protection, and location 6 is around and inside the port. 


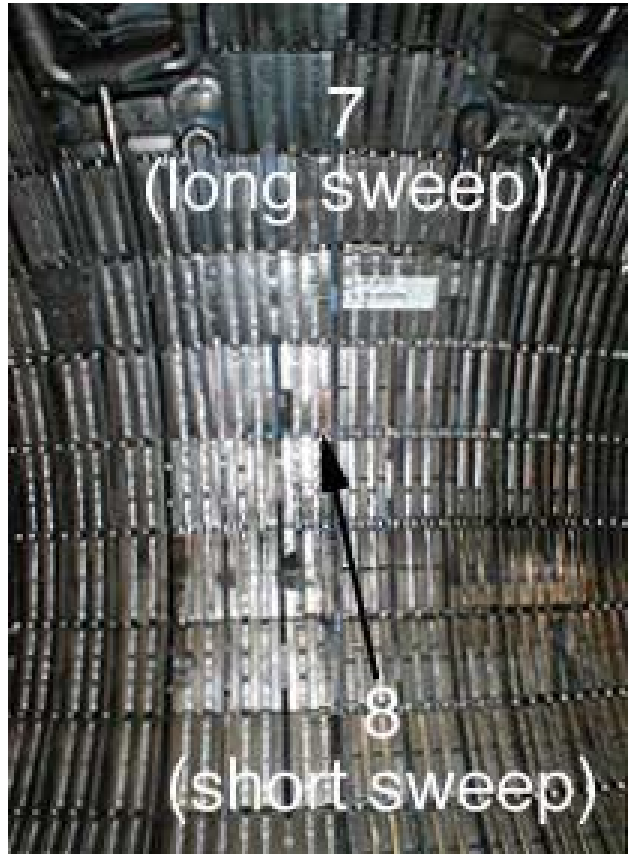

Figure 2.6 Poloidal sampling locations 7 and 8. Location 7 is a sweep along the entire column height, and location 8 is a short, midplane sweep.

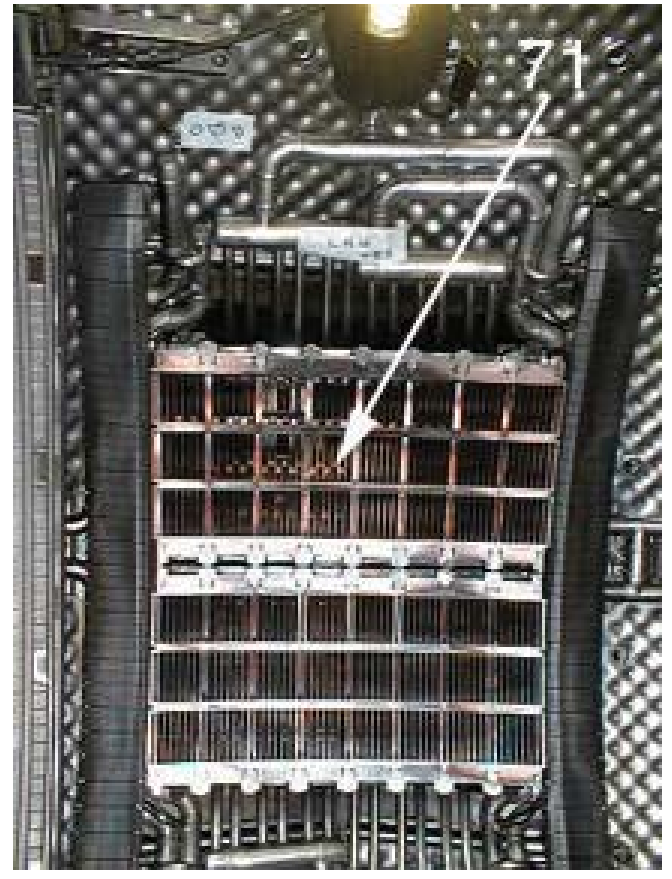

Figure 2.7 LHH launcher guides- sampling location 71 .

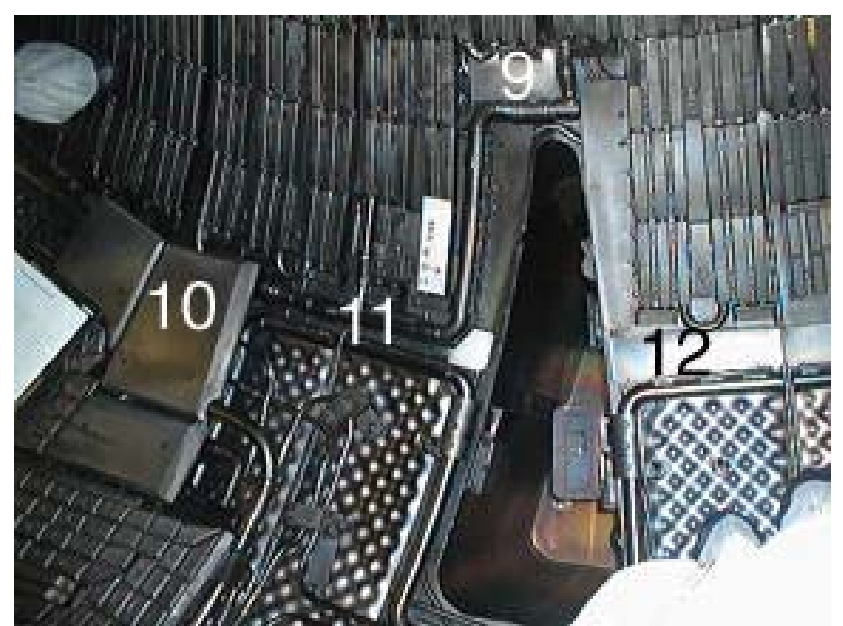

Figure 2.8 Poloidal sampling locations 9, 10,11 , and 12 .

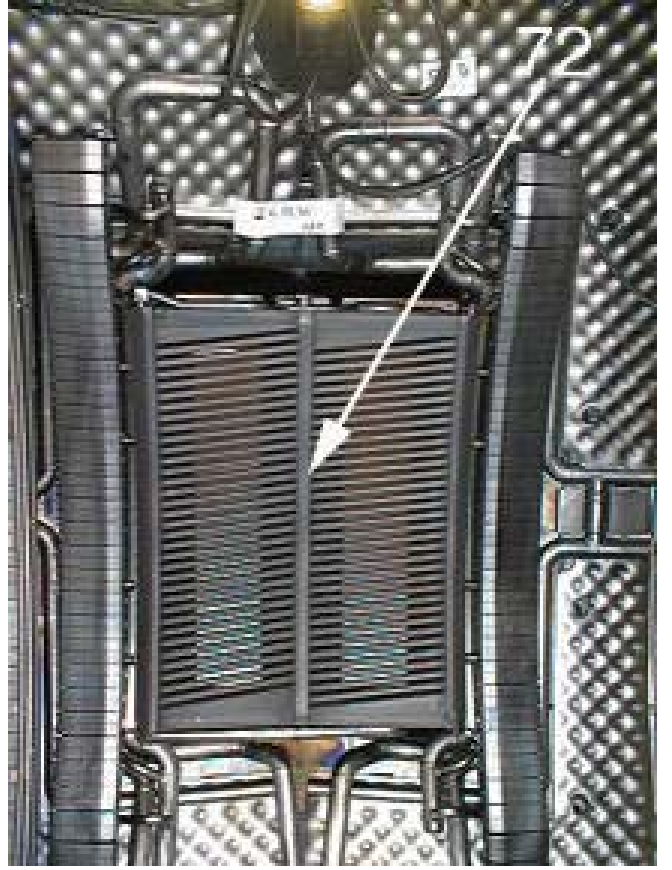

Figure 2.9 ICRH Faraday shield- sampling location 72 . 


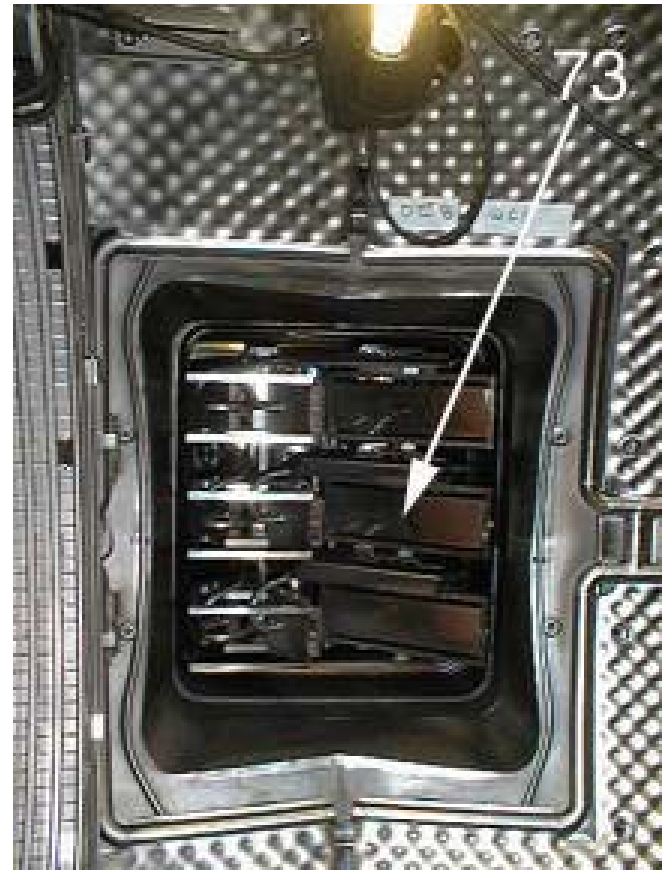

Figure 2.10 ECRH antenna mirrorssampling location 73 .

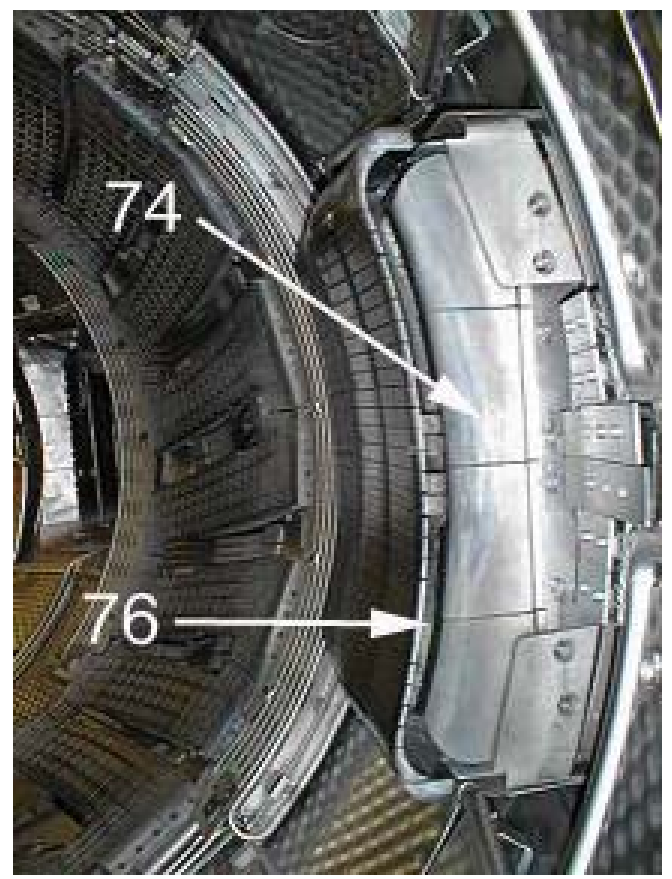

Figure 2.11 Detail of horizontal pump limiter ionic side- sampling locations 74 (throat) and 76 (face). Sampling locations 75 and 77 are the corresponding electronic positions on the opposite side.

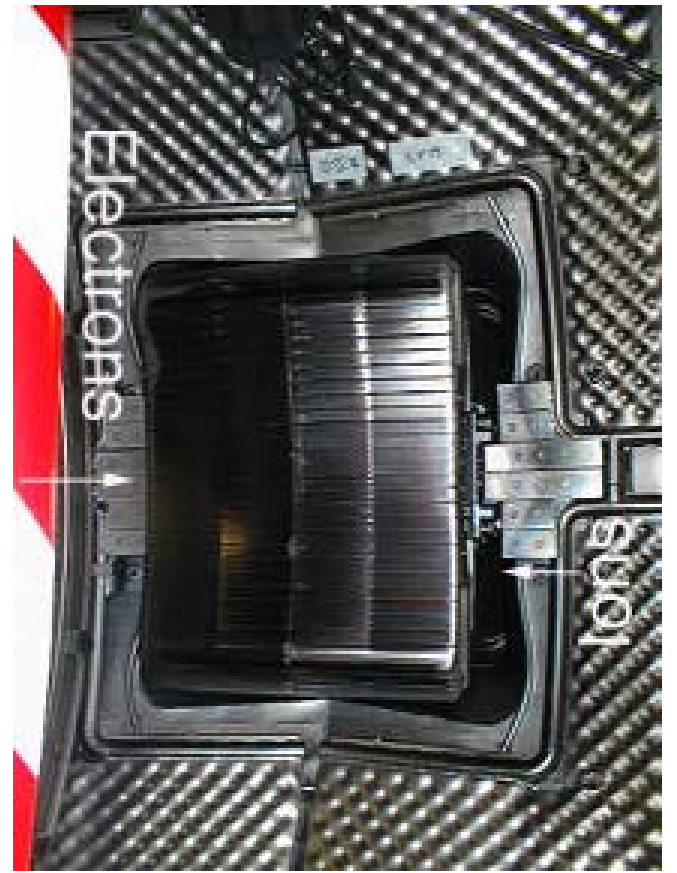

Figure 2.12 Horizontal pump limiter. In this view, the right side of the limiter is the ionic face, and the left side is the electronic face. 


\section{Particle Size Analysis}

Two different techniques were utilized in determining particle size distributions of material collected via the filter vacuum technique: optical microscopy imaging and particle counting, and laser diffraction particle analysis. Optical microscopy image and count analysis served as the primary technique, and each filter substrate used for collection in Tore Supra was analyzed in detail with this method. Laser diffraction analysis is a powerful tool, however difficulties were encountered in sample preparation due to the requirement of particle removal from the filter substrates. In addition to size distribution analyses, particulate morphology (shape, degree of agglomeration or clustering, cleaved surfaces, etc.) was examined for a qualitative description of the state of the particulate.

\subsection{Particulate Morphology}

Details of particle morphology were investigated by selective imaging of filter substrates with a scanning electron microscope (SEM). The SEM is capable of producing high resolution and very sharp images, as shown in the representative photomicrographs in Figure 3.1. Typical particles found in all filter vacuum samples are shown in the figure; the dust sample in the figure was collected from the area around the upper observation port ripple protection (poloidal location 5) in Module I. Figure 3.1(a) shows a moderate-sized carbon flake, small fibers or agglomerate chains (difficult to distinguish in this image), and a cleaved particle of alumina. Figure 3.1(b) displays a spherical copper particle and clusters with very small particles of undetermined composition. Variety of particle shapes indicates several mechanisms are responsible for the generation of dust in Tore Supra. Observations of dust from several samples collected at various locations in the vessel suggest that a predominant number of particles appear as small clusters of very small particles.

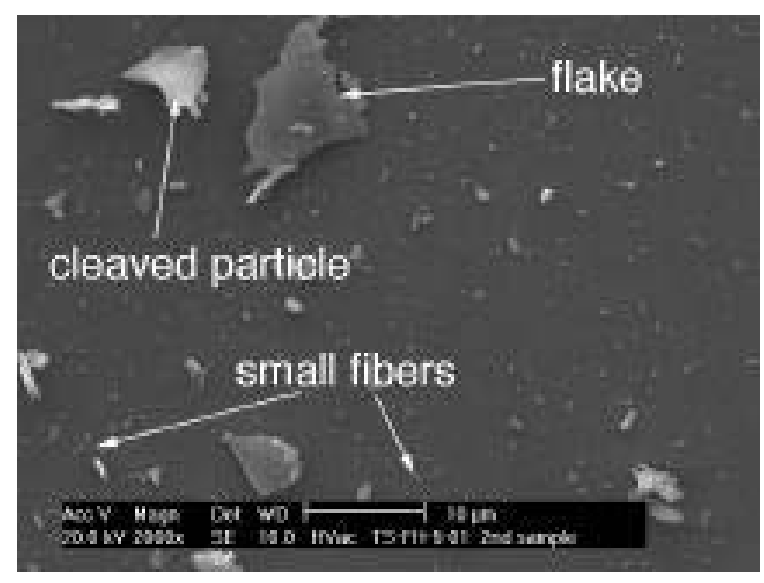

(a)

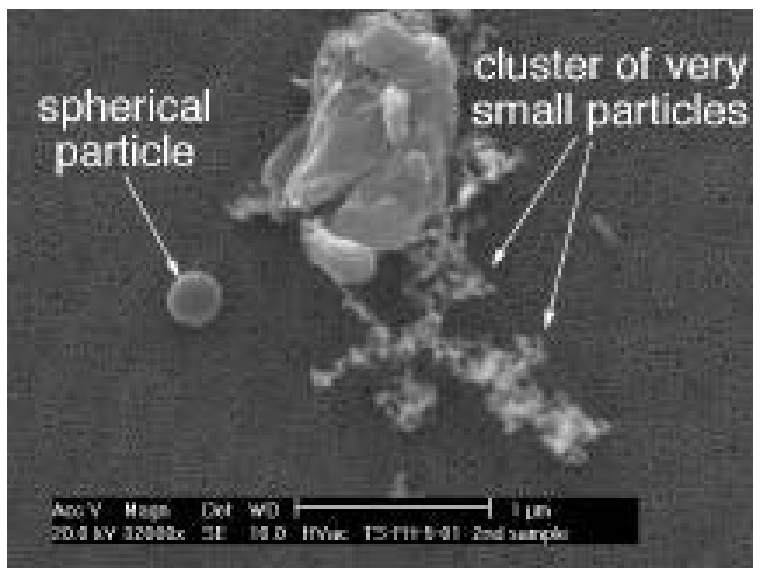

(b)

Figure 3.1 Representative SEM photomicrographs demonstrating various particle morphologies observed in dust samples from Tore Supra. 
Another qualitative imaging analysis of a dust sample was performed with a tunneling electron microscope (TEM). The TEM is capable of very high resolution, although image quality is sometimes poor due to inadequate methods of sample preparation. The dust sample analyzed with a TEM at the INEEL was collected from a graphite tile on the inner first wall, one row below the midplane in Module V. Metallurgical replicating tape (an acetate compound) was used to literally lift off particles adhered to the tile surface. This collection technique is useful for acquiring samples from small areas since all particle sizes are collected, particularly particles below the collection limit of the vacuum filter method (roughly $0.1 \mu \mathrm{m}$ ). Sample preparation for TEM analysis consisted of several steps:

1.0 dissolution of the acetate tape in a small vial containing acetone

2.0 capture of suspended particulate in the acetone solution onto a TEM analysis grid

3.0 drying of the grid and evaporation of the acetone/acetate solution

4.0 cleaning of the grid with acetone to remove trace amounts of acetate

5.0 grid mounting in the TEM

The presence of acetate compounds on the analysis grid severely deteriorates the imaging capability of the TEM at the INEEL. Step 4 in the preparation procedure was required to allow generation of reasonable images. Figure 3.2 gives two images acquired with the TEM. Although the quality is very poor, the images display discrete rounded structures. Similar structures have been observed by Chappuis, et al., in Tore Supra dust ${ }^{4}$, along with extended filamentary structures reminiscent of carbon nanotubes. A more suitable particle collection technique must be used in future attempts to collect and characterize these tokamak nanostructures.

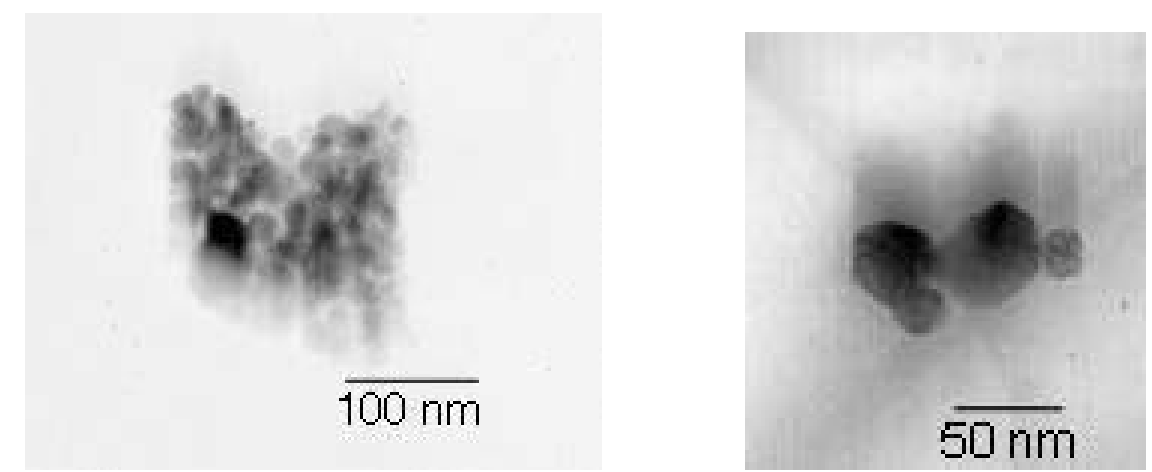

Figure 3.2 TEM photomicrographs of dust collected with replicating tape.

\subsection{Size Analysis by Optical Microscopy}

\subsubsection{Technique}

Dust collected from Tore Supra via the filtered vacuum method has been analyzed for countbased particle size distributions using the procedure described in Reference 3. The procedure requires photomicrographs of multiple, randomly selected sites on the filter substrate from each sampling location. Photomicrographs are obtained with an optical microscope at magnifications 
of 50x, 100x, 200x, 500x, and 1000x. An automated sizing and counting routine, using the freeware image analysis program NIHImage ${ }^{5}$, is used to develop the particle sample population. The reported size of each tallied particle is the equivalent diameter of the projected particle area as observed at the focal plane of the microscope. From this population, the data moments of geometric mean diameter (GMD) and geometric standard deviation (GSD) are acquired. Geometric moments are typically used because of the direct relation to the lognormal distribution. The measured population is compared to a lognormal distribution by use of a logprobability plot, on which a lognormal distribution is linear. If the sample distribution is truly lognormal, the particle size data count median diameter (CMD) is equivalent to the GMD. A measure of comparison is the linear correlation coefficient $\mathrm{R}^{2}$ associated with a linear fit of the data. Departure from linearity, i.e. values of $\mathrm{R}^{2}$ less than 0.99 , indicates a sample population that is not best described by the unimodal lognormal distribution.

\subsubsection{Results}

This section presents the results of the optical microscopy analysis for each filter substrate from all collection locations in Tore Supra. A summary table for each toroidal module is presented in the following subsections, reporting the amount of mass collected on from each sample location, the area sampled, and the resulting count-based size distribution data moments (GMD and GSD). The total dust mass that was collected during this campaign is $439.2 \mathrm{mg}$.

\subsubsection{Module I Results}

Figure 3.3 through Figure 3.14 display details of particle size distributions for corresponding collection locations of Module I. Table 3.1 gives a summary of Module I results.

Table 3.1 Summary of dust collected from locations in Tore Supra Module I.

\begin{tabular}{|c|c|c|c|c|c|c|}
\hline \multirow{2}{*}{$\begin{array}{c}\text { Poloidal } \\
\text { Location }\end{array}$} & $\begin{array}{c}\text { Sample } \\
\text { Number }\end{array}$ & Filter ID & $\begin{array}{c}\text { Sampled } \\
\text { Area }\left(\mathrm{cm}^{2}\right)\end{array}$ & $\begin{array}{c}\text { Collected } \\
\text { Mass }(\mathrm{mg})\end{array}$ & \multicolumn{2}{|c|}{$\begin{array}{c}\text { Count-based Size } \\
\text { Distribution Parameters }\end{array}$} \\
\hline \hline 1 & 1 & TSFH22-01 & 621.2 & 21.9 & 2.23 & 2.79 \\
\hline 2 & 2 & TSFH01-01 & 1,553 & 0.8 & 3.08 & 3.25 \\
\hline 4 & 3 & TSFH23-01 & 689.0 & 0.4 & 3.00 & 2.87 \\
\hline 3 & 4 & TSFH24-01 & 1,800 & 0.2 & 3.13 & 2.65 \\
\hline 6 & 5 & TSFH25-01 & 658.0 & 0.0 & 3.30 & 2.79 \\
\hline 5 & 6 & TSFH05-01 & 1,200 & 1.0 & 2.59 & 2.92 \\
\hline 7 & 7 & TSFH07-01 & 428.0 & 3.8 & 2.17 & 2.65 \\
\hline 8 & 8 & TSFH06-01 & 84.00 & 0.2 & 1.83 & 3.12 \\
\hline 10 & 9 & TSFH04-01 & 1,429 & 0.8 & 3.10 & 2.72 \\
\hline 9 & 10 & TSFH02-01 & 294.0 & 0.1 & 3.13 & 2.67 \\
\hline 11 & $10 \mathrm{~A}$ & TSFH21-01 & 160.0 & 34.5 & 1.30 & 2.43 \\
\hline 12 & $10 \mathrm{~B}$ & TSFH03-01 & 480.0 & 13.9 & 2.02 & 2.83 \\
\hline
\end{tabular}

\footnotetext{
*- filter partially broken
} 


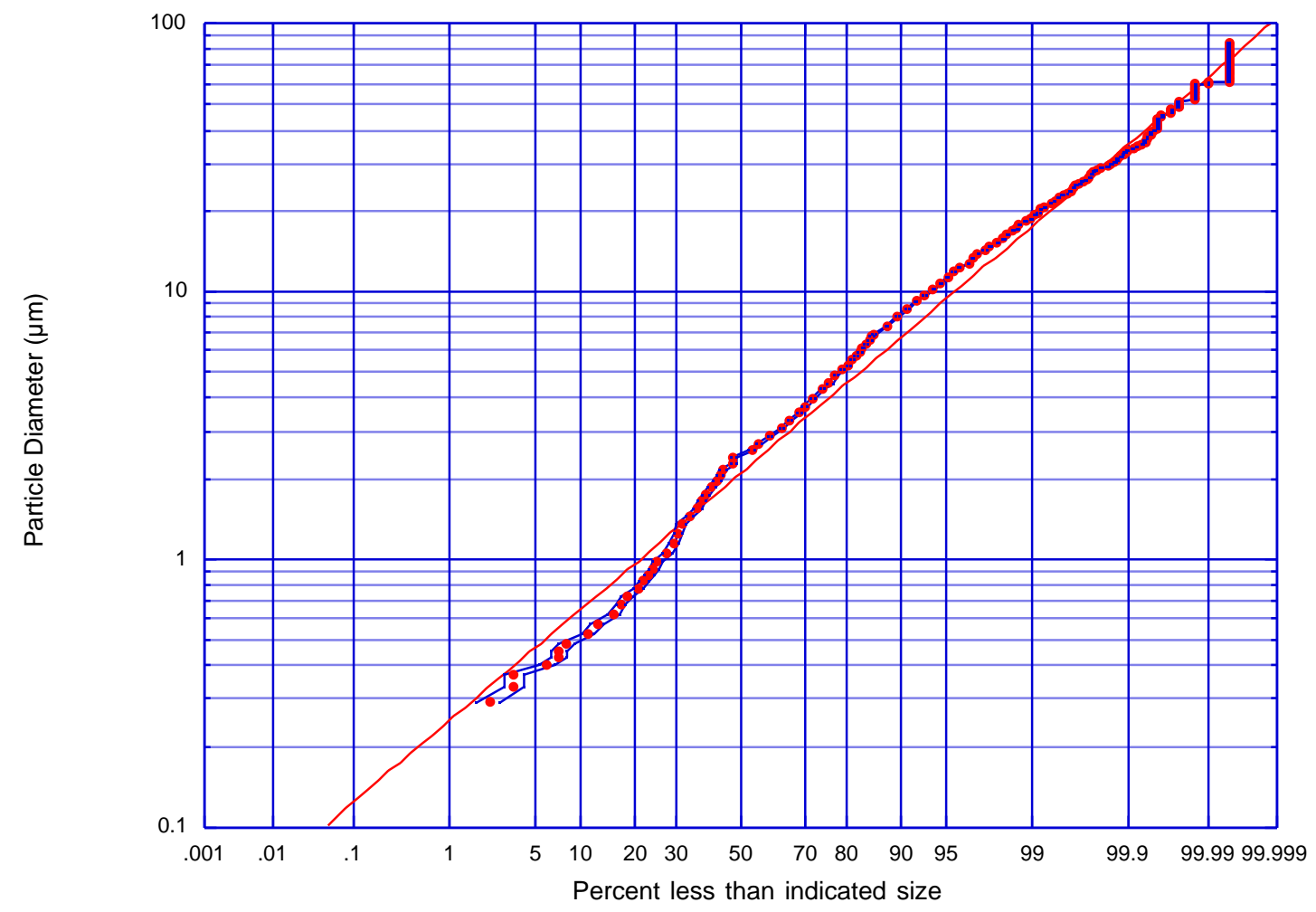

Analysis Summary Table:

\begin{tabular}{|c|c|c|c|c|c|}
\hline \multicolumn{2}{|c|}{$\begin{array}{c}\text { magnification, } \\
\text { number of images }\end{array}$} & \multicolumn{2}{|c|}{ area analyzed $^{1}(\%)$} & counted size range $(\mu \mathrm{m})$ & $\begin{array}{c}\text { total number of } \\
\text { particles (not scaled) }\end{array}$ \\
\hline $50 x$ & 6 & \multicolumn{2}{|r|}{0.894} & $d_{p}>7.01$ & 4101 \\
\hline $100 x$ & 6 & \multicolumn{2}{|r|}{0.224} & $3.82<d_{p}<7.01$ & 3574 \\
\hline $200 x$ & 6 & \multicolumn{2}{|r|}{0.0558} & $2.29<d_{p}<3.82$ & 3398 \\
\hline $500 x$ & 6 & \multicolumn{2}{|r|}{0.00893} & $1.40<\mathrm{d}_{\mathrm{p}}<2.29$ & 1105 \\
\hline 1000x, & 6 & \multicolumn{2}{|r|}{0.00217} & $0.00<\mathrm{d}_{\mathrm{p}}<1.40$ & 450 \\
\hline \multicolumn{2}{|c|}{ Totals: } & \multicolumn{2}{|r|}{$1.18 \%$} & - & 12628 \\
\hline \multicolumn{4}{|c|}{ data moments ${ }^{2}:$} & \multicolumn{2}{|c|}{ log-normal fit moments ${ }^{2}$ : } \\
\hline \multicolumn{3}{|c|}{ GMD $(\mu \mathrm{m}):$} & 2.23 & GMD $(\mu \mathrm{m}):$ & 2.09 \\
\hline \multicolumn{2}{|c|}{ GSD: } & & 2.79 & GSD: & 2.50 \\
\hline \multicolumn{3}{|c|}{ lower $95 \%(\mu \mathrm{m})$ : } & 0.29 & $\mathrm{R}^{2}$ & 0.99439 \\
\hline \multirow{2}{*}{\multicolumn{3}{|c|}{ upper $95 \%(\mu \mathrm{m})$ : }} & 17.28 & lower $95 \%(\mu \mathrm{m})$ : & 0.34 \\
\hline & & & & upper $95 \%(\mu \mathrm{m})$ : & 13.07 \\
\hline
\end{tabular}

1. based on overall filter area of $1.59 \times 10^{9} \mu \mathrm{m}^{2}$

2. $\mathrm{GMD}=$ geometric mean diameter, $\mathrm{GSD}=$ geometric standard deviation, $\mathrm{R}=$ linear correlation coefficient

Figure 3.3 Module I, Sample 1 (TSFH22-01) count-based particle size distribution. 


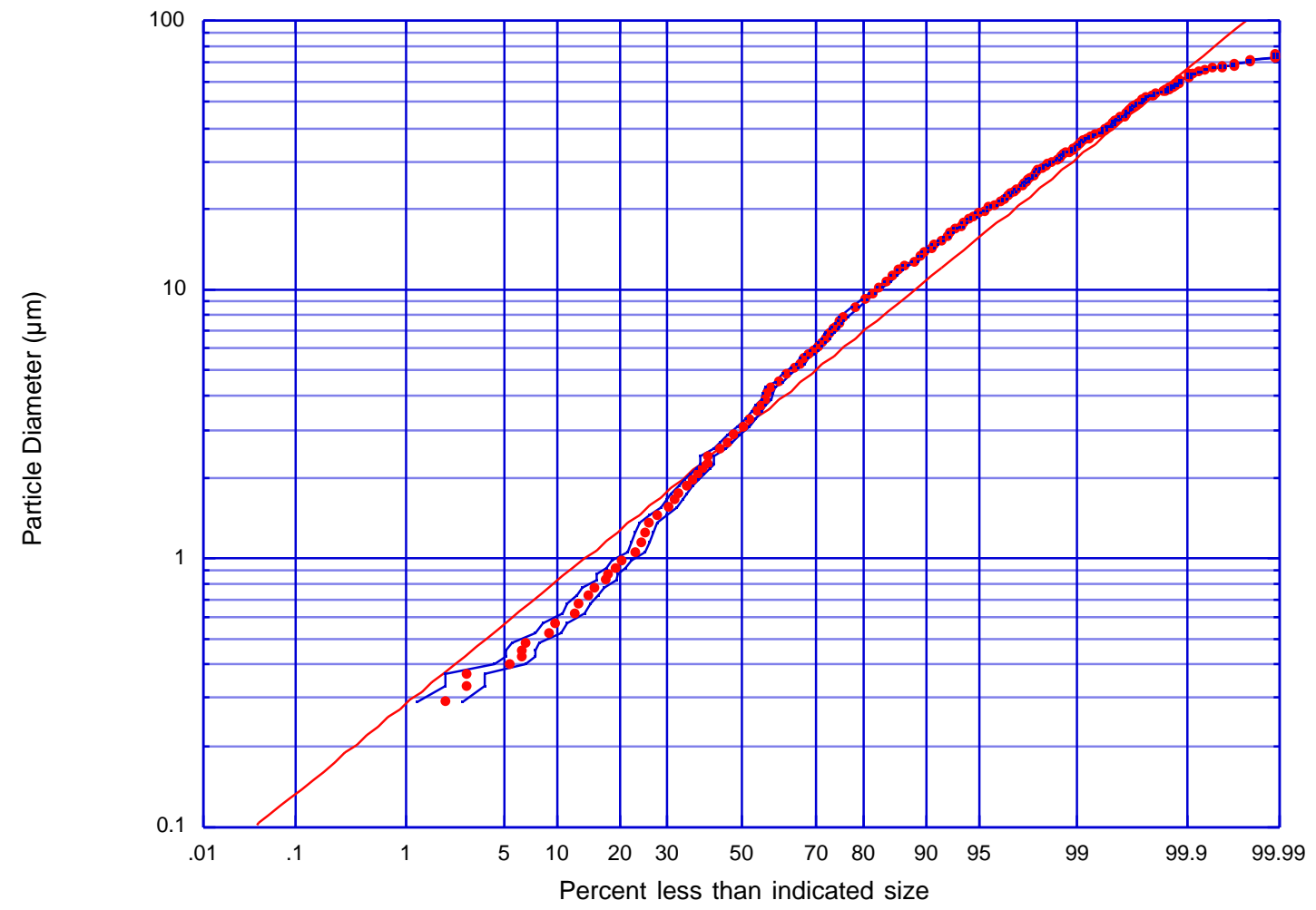

Analysis Summary Table:

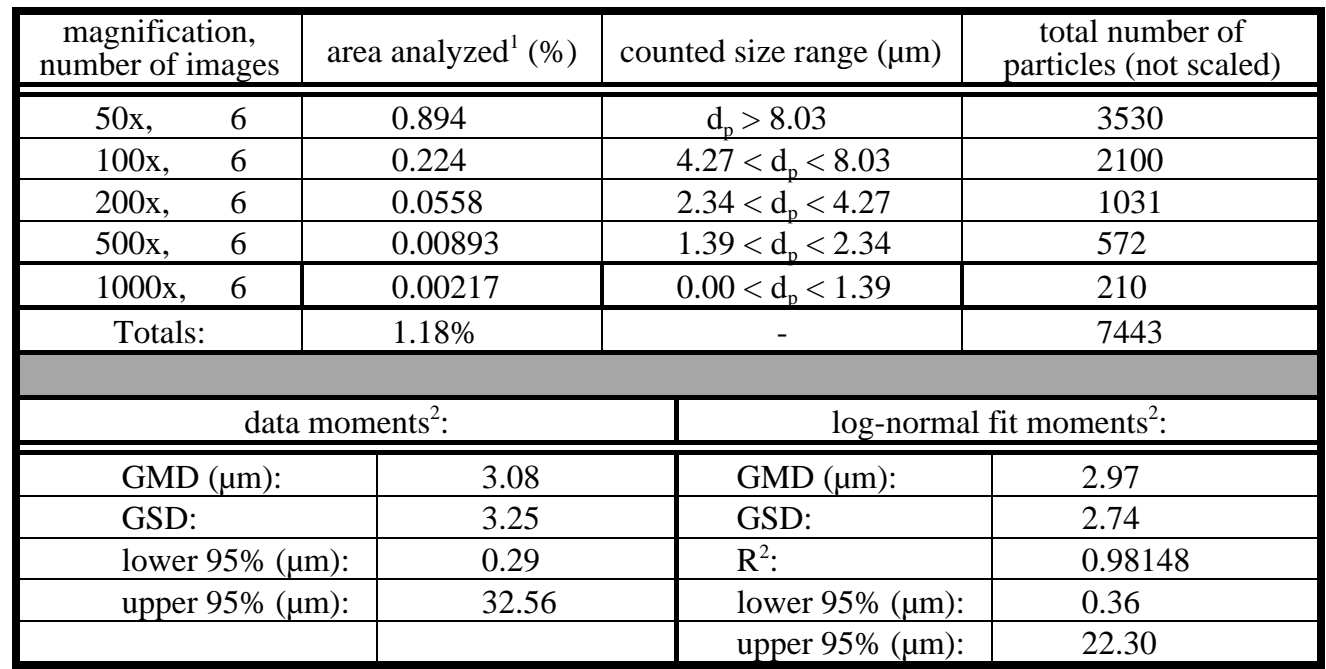

1. based on overall filter area of $1.59 \times 10^{9} \mu \mathrm{m}^{2}$

2. $\mathrm{GMD}=$ geometric mean diameter, $\mathrm{GSD}=$ geometric standard deviation, $\mathrm{R}=$ linear correlation coefficient

Figure 3.4 Module I, Sample 2 (TSFH01-01) count-based particle size distribution. 


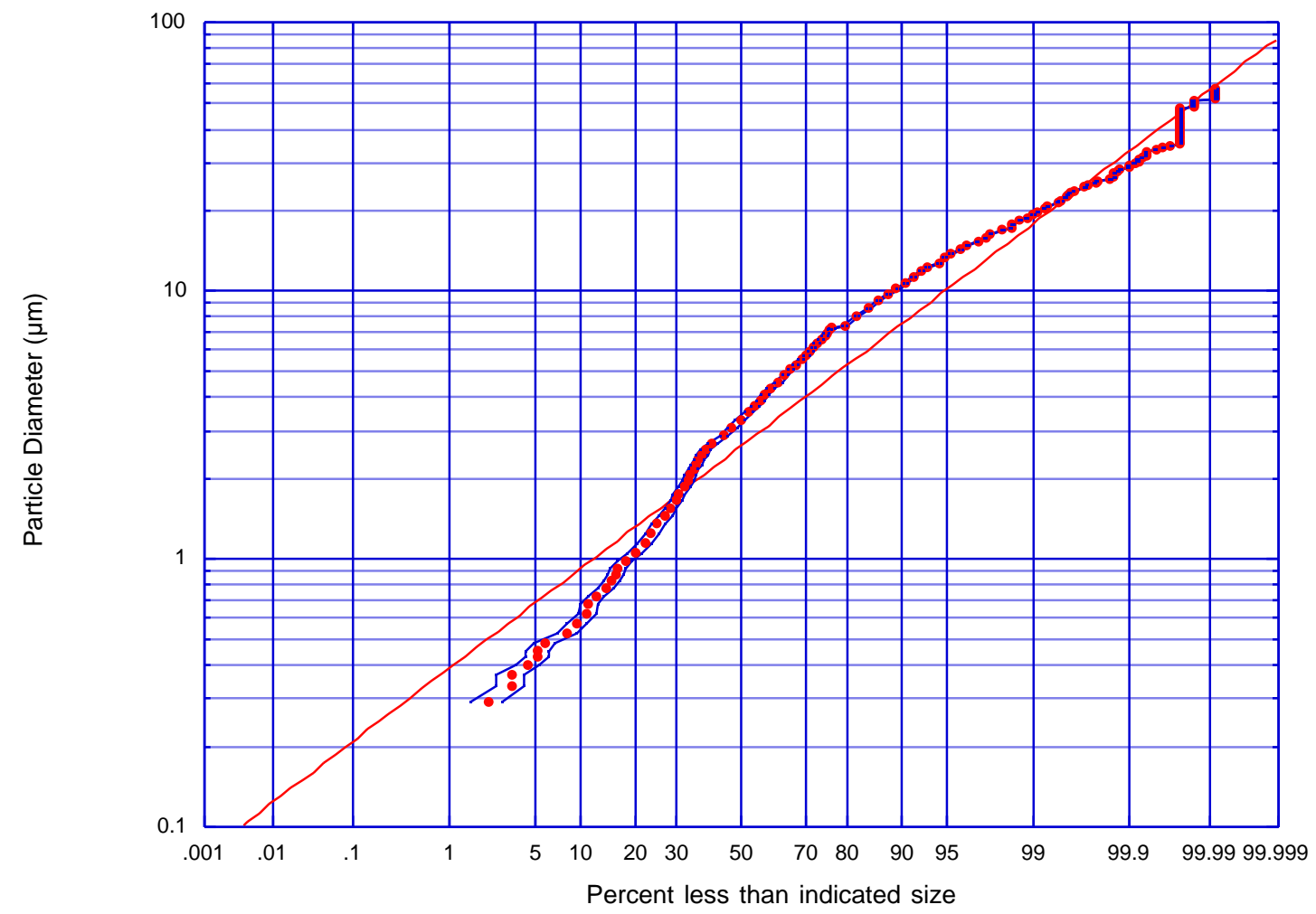

Analysis Summary Table:

\begin{tabular}{|c|c|c|c|c|c|}
\hline \multicolumn{2}{|c|}{$\begin{array}{l}\text { magnification, } \\
\text { number of images }\end{array}$} & \multicolumn{2}{|c|}{ area analyzed $^{1}(\%)$} & counted size range $(\mu \mathrm{m})$ & $\begin{array}{c}\text { total number of } \\
\text { particles (not scaled) }\end{array}$ \\
\hline $50 x$, & 6 & \multicolumn{2}{|r|}{0.894} & $d_{p}>7.47$ & 3982 \\
\hline $100 x$ & 6 & \multicolumn{2}{|r|}{0.224} & $4.50<d_{p}<7.47$ & 3107 \\
\hline 200x, & 6 & \multicolumn{2}{|r|}{0.0558} & $2.66<d_{p}<4.50$ & 2080 \\
\hline $500 x$ & 6 & \multicolumn{2}{|r|}{0.00893} & $1.61<d_{p}<2.66$ & 489 \\
\hline $1000 x$ & 6 & \multicolumn{2}{|r|}{0.00217} & $0.00<d_{p}<1.61$ & 279 \\
\hline \multicolumn{2}{|c|}{ Totals: } & \multicolumn{2}{|r|}{$1.18 \%$} & - & 9937 \\
\hline \multicolumn{4}{|c|}{ data moments ${ }^{2}$ : } & \multicolumn{2}{|c|}{ log-normal fit moments ${ }^{2}$ : } \\
\hline \multicolumn{3}{|c|}{ GMD $(\mu \mathrm{m}):$} & 3.00 & GMD $(\mu \mathrm{m})$ : & 2.64 \\
\hline \multicolumn{2}{|c|}{ GSD: } & & 2.87 & GSD: & 2.27 \\
\hline \multicolumn{3}{|c|}{ lower $95 \%(\mu \mathrm{m})$ : } & 0.37 & $\mathrm{R}^{2}:$ & 0.97507 \\
\hline \multirow{2}{*}{\multicolumn{3}{|c|}{ upper $95 \%(\mu \mathrm{m})$ : }} & 24.65 & lower $95 \%(\mu \mathrm{m})$ : & 0.51 \\
\hline & & & & upper $95 \%(\mu \mathrm{m})$ : & 13.64 \\
\hline
\end{tabular}

1. based on overall filter area of $1.59 \times 10^{9} \mu \mathrm{m}^{2}$

2. $\mathrm{GMD}=$ geometric mean diameter, GSD = geometric standard deviation, $\mathrm{R}=$ linear correlation coefficient

Figure 3.5 Module I, Sample 3 (TSFH23-01) count-based particle size distribution. 


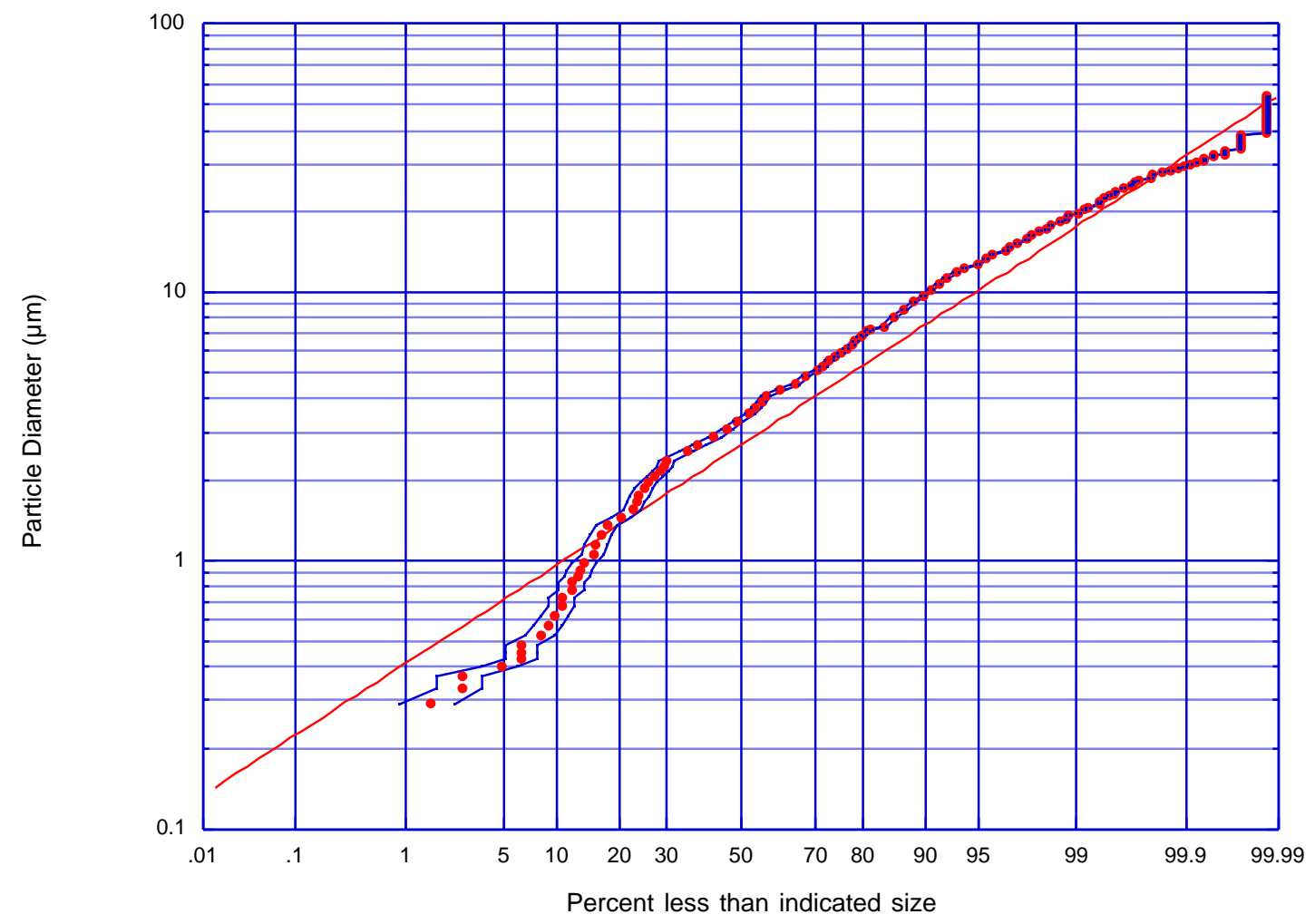

Analysis Summary Table:

\begin{tabular}{|c|c|c|c|c|c|}
\hline \multicolumn{2}{|c|}{$\begin{array}{c}\text { magnification, } \\
\text { number of images }\end{array}$} & \multicolumn{2}{|c|}{ area analyzed $^{1}(\%)$} & counted size range $(\mu \mathrm{m})$ & $\begin{array}{c}\text { total number of } \\
\text { particles (not scaled) }\end{array}$ \\
\hline $50 x$ & 6 & \multicolumn{2}{|r|}{0.894} & $d_{p}>7.45$ & 1800 \\
\hline $100 x$ & 6 & \multicolumn{2}{|r|}{0.224} & $4.22<d_{p}<7.45$ & 2354 \\
\hline $200 x$ & 6 & \multicolumn{2}{|r|}{0.0558} & $2.48<\mathrm{d}_{\mathrm{p}}<4.22$ & 1252 \\
\hline $500 x$ & 6 & \multicolumn{2}{|r|}{0.00893} & $1.44<\mathrm{d}_{\mathrm{p}}<2.48$ & 307 \\
\hline 1000x, & 6 & \multicolumn{2}{|r|}{0.00217} & $0.00<\mathrm{d}_{\mathrm{p}}<1.44$ & 114 \\
\hline \multicolumn{2}{|c|}{ Totals: } & \multicolumn{2}{|r|}{$1.18 \%$} & - & 5827 \\
\hline \multicolumn{4}{|c|}{ data moments ${ }^{2}:$} & \multicolumn{2}{|c|}{ log-normal fit moments ${ }^{2}$ : } \\
\hline \multicolumn{3}{|c|}{ GMD $(\mu \mathrm{m}):$} & 3.13 & GMD $(\mu \mathrm{m}):$ & 2.70 \\
\hline \multicolumn{2}{|c|}{ GSD: } & & 2.65 & GSD: & 2.24 \\
\hline \multicolumn{3}{|c|}{ lower $95 \%(\mu \mathrm{m})$ : } & 0.45 & $\mathrm{R}^{2}$ & 0.97778 \\
\hline \multirow{2}{*}{\multicolumn{3}{|c|}{ upper $95 \%(\mu \mathrm{m})$ : }} & 21.88 & lower $95 \%(\mu \mathrm{m})$ : & 0.54 \\
\hline & & & & upper $95 \%(\mu \mathrm{m})$ : & 13.51 \\
\hline
\end{tabular}

1. based on overall filter area of $1.59 \times 10^{9} \mu \mathrm{m}^{2}$

2. $\mathrm{GMD}=$ geometric mean diameter, $\mathrm{GSD}=$ geometric standard deviation, $\mathrm{R}=$ linear correlation coefficient

Figure 3.6 Module I, Sample 4 (TSFH24-01) count-based particle size distribution. 


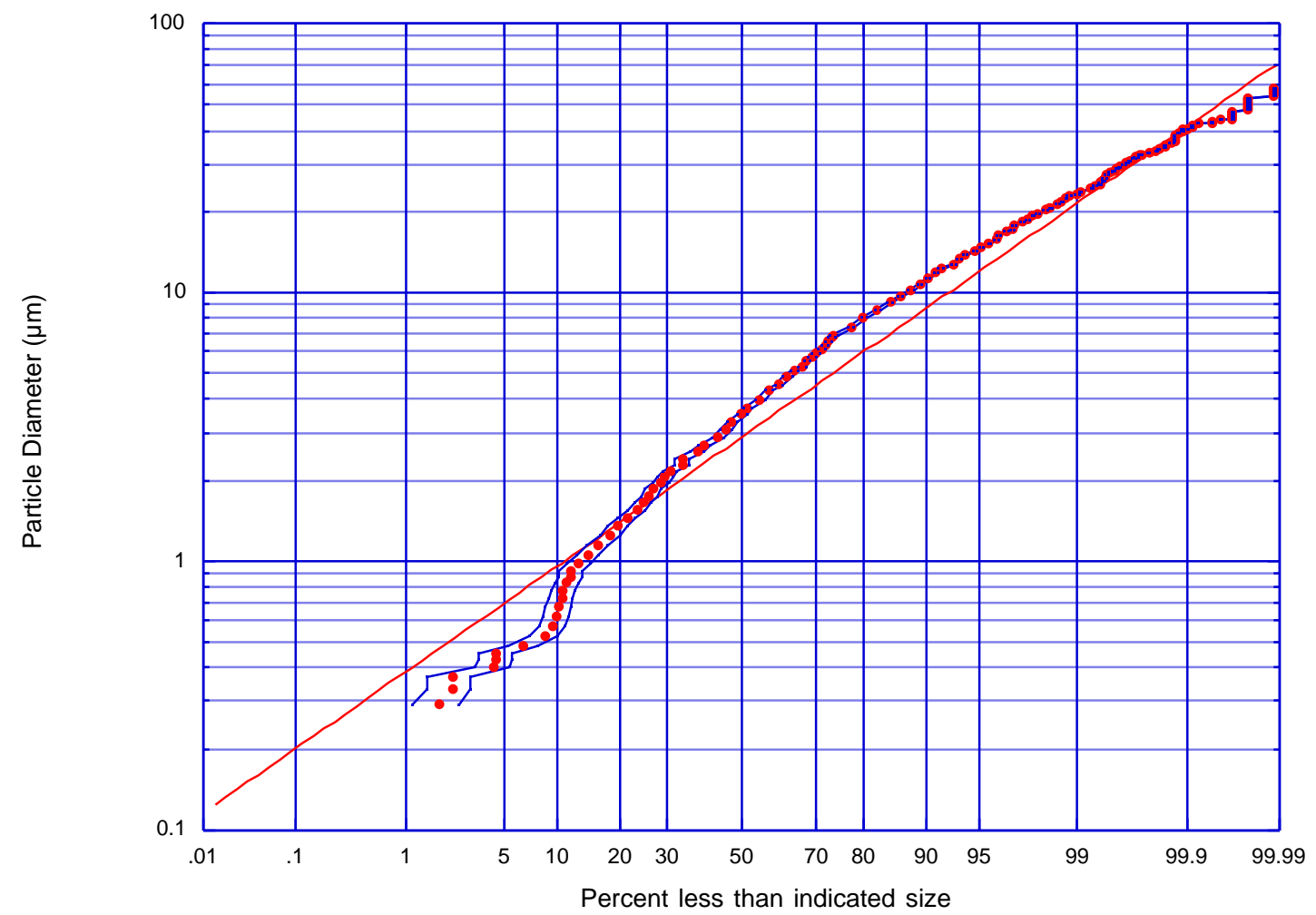

Analysis Summary Table:

\begin{tabular}{|c|c|c|c|c|c|}
\hline \multicolumn{2}{|c|}{$\begin{array}{c}\text { magnification, } \\
\text { number of images }\end{array}$} & \multicolumn{2}{|c|}{ area analyzed $^{1}(\%)$} & counted size range $(\mu \mathrm{m})$ & $\begin{array}{c}\text { total number of } \\
\text { particles (not scaled) }\end{array}$ \\
\hline $50 x$ & 6 & \multicolumn{2}{|r|}{0.894} & $d_{p}>7.16$ & 3161 \\
\hline $100 x$ & 6 & \multicolumn{2}{|r|}{0.224} & $3.90<d_{p}<7.16$ & 2056 \\
\hline $200 x$ & 6 & \multicolumn{2}{|r|}{0.0558} & $2.22<d_{p}<3.90$ & 1139 \\
\hline $500 x$ & 6 & \multicolumn{2}{|r|}{0.00893} & $1.28<\mathrm{d}_{\mathrm{p}}<2.22$ & 355 \\
\hline 1000x, & 6 & \multicolumn{2}{|r|}{0.00217} & $0.00<\mathrm{d}_{\mathrm{p}}<1.28$ & 124 \\
\hline \multicolumn{2}{|c|}{ Totals: } & \multicolumn{2}{|r|}{$1.18 \%$} & - & 6835 \\
\hline \multicolumn{4}{|c|}{ data moments ${ }^{2}:$} & \multicolumn{2}{|c|}{ log-normal fit moments ${ }^{2}$ : } \\
\hline \multicolumn{3}{|c|}{ GMD $(\mu \mathrm{m}):$} & 3.30 & GMD $(\mu \mathrm{m}):$ & 2.89 \\
\hline \multicolumn{2}{|c|}{ GSD: } & & 2.79 & GSD: & 2.37 \\
\hline \multicolumn{3}{|c|}{ lower $95 \%(\mu \mathrm{m})$ : } & 0.42 & $\mathrm{R}^{2}$ & 0.9821 \\
\hline \multirow{2}{*}{\multicolumn{3}{|c|}{ upper $95 \%(\mu \mathrm{m})$ : }} & 25.70 & lower $95 \%(\mu \mathrm{m})$ : & 0.51 \\
\hline & & & & upper $95 \%(\mu \mathrm{m})$ : & 16.27 \\
\hline
\end{tabular}

1. based on overall filter area of $1.59 \times 10^{9} \mu \mathrm{m}^{2}$

2. $\mathrm{GMD}=$ geometric mean diameter, $\mathrm{GSD}=$ geometric standard deviation, $\mathrm{R}=$ linear correlation coefficient

Figure 3.7 Module I, Sample 5 (TSFH25-01) count-based particle size distribution. 


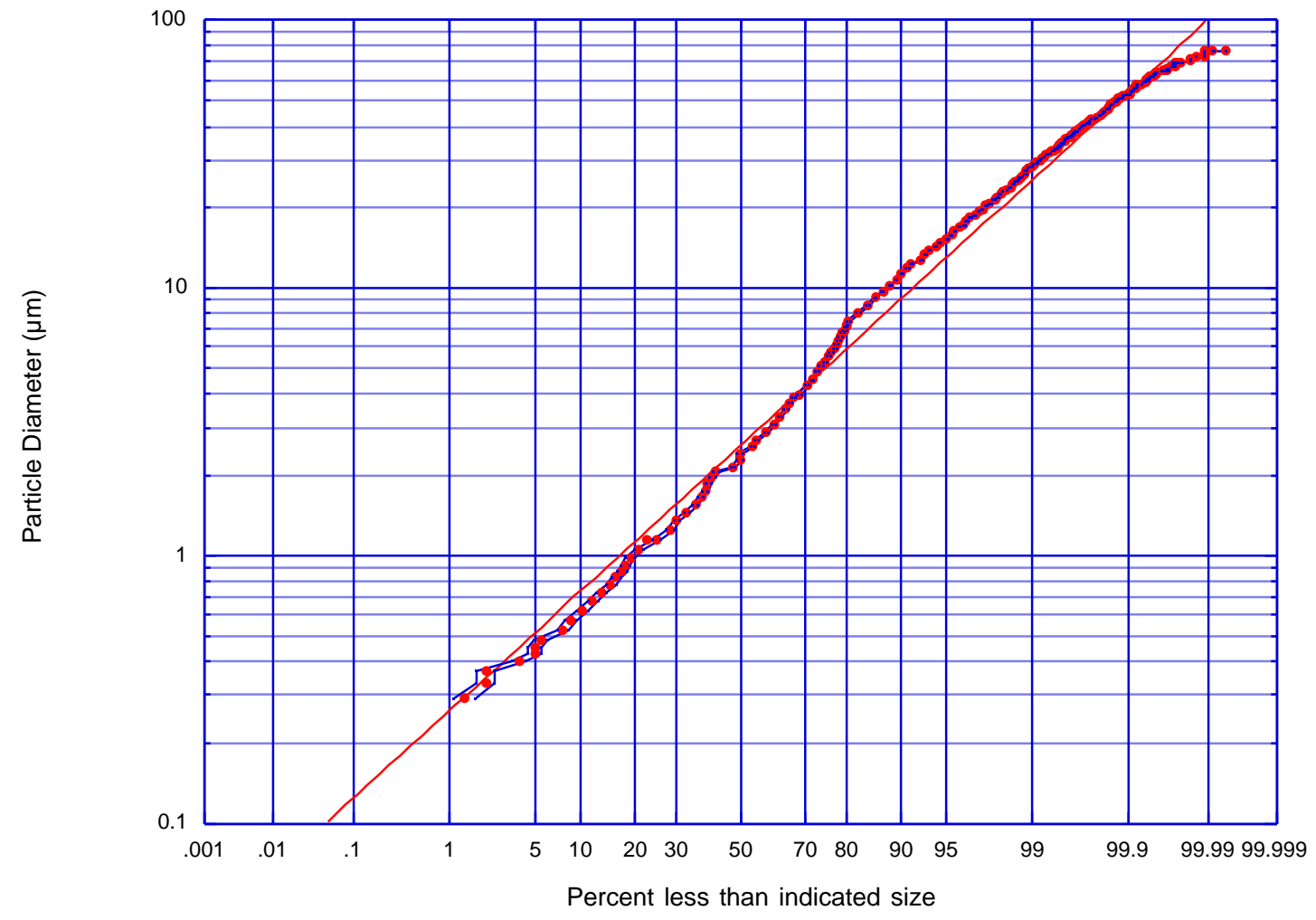

Analysis Summary Table:

\begin{tabular}{|c|c|c|c|c|c|}
\hline \multicolumn{2}{|c|}{$\begin{array}{c}\text { magnification, } \\
\text { number of images }\end{array}$} & \multicolumn{2}{|c|}{ area analyzed $^{1}(\%)$} & counted size range $(\mu \mathrm{m})$ & $\begin{array}{c}\text { total number of } \\
\text { particles (not scaled) }\end{array}$ \\
\hline $50 x$ & 6 & \multicolumn{2}{|r|}{0.894} & $d_{p}>7.74$ & 10475 \\
\hline $100 x$ & 6 & \multicolumn{2}{|r|}{0.224} & $4.11<d_{p}<7.74$ & 5374 \\
\hline $200 x$ & 6 & \multicolumn{2}{|r|}{0.0558} & $2.17<\mathrm{d}_{\mathrm{p}}<4.11$ & 4252 \\
\hline $500 x$ & 6 & \multicolumn{2}{|r|}{0.00893} & $1.21<\mathrm{d}_{\mathrm{p}}<2.17$ & 1828 \\
\hline 1000x, & 6 & \multicolumn{2}{|r|}{0.00217} & $0.00<\mathrm{d}_{\mathrm{p}}<1.21$ & 621 \\
\hline \multicolumn{2}{|c|}{ Totals: } & \multicolumn{2}{|r|}{$1.18 \%$} & - & 22550 \\
\hline \multicolumn{4}{|c|}{ data moments ${ }^{2}:$} & \multicolumn{2}{|c|}{ log-normal fit moments ${ }^{2}$ : } \\
\hline \multicolumn{3}{|c|}{ GMD $(\mu \mathrm{m}):$} & 2.59 & GMD $(\mu \mathrm{m}):$ & 2.59 \\
\hline \multicolumn{2}{|c|}{ GSD: } & & 2.92 & GSD: & 2.67 \\
\hline \multicolumn{3}{|c|}{ lower $95 \%(\mu \mathrm{m})$ : } & 0.30 & $\mathrm{R}^{2}$ & 0.99254 \\
\hline \multirow{2}{*}{\multicolumn{3}{|c|}{ upper $95 \%(\mu \mathrm{m})$ : }} & 22.01 & lower $95 \%(\mu \mathrm{m})$ : & 0.36 \\
\hline & & & & upper $95 \%(\mu \mathrm{m})$ : & 18.47 \\
\hline
\end{tabular}

1. based on overall filter area of $1.59 \times 10^{9} \mu \mathrm{m}^{2}$

2. $\mathrm{GMD}=$ geometric mean diameter, $\mathrm{GSD}=$ geometric standard deviation, $\mathrm{R}=$ linear correlation coefficient

Figure 3.8 Module I, Sample 6 (TSFH05-01) count-based particle size distribution. 


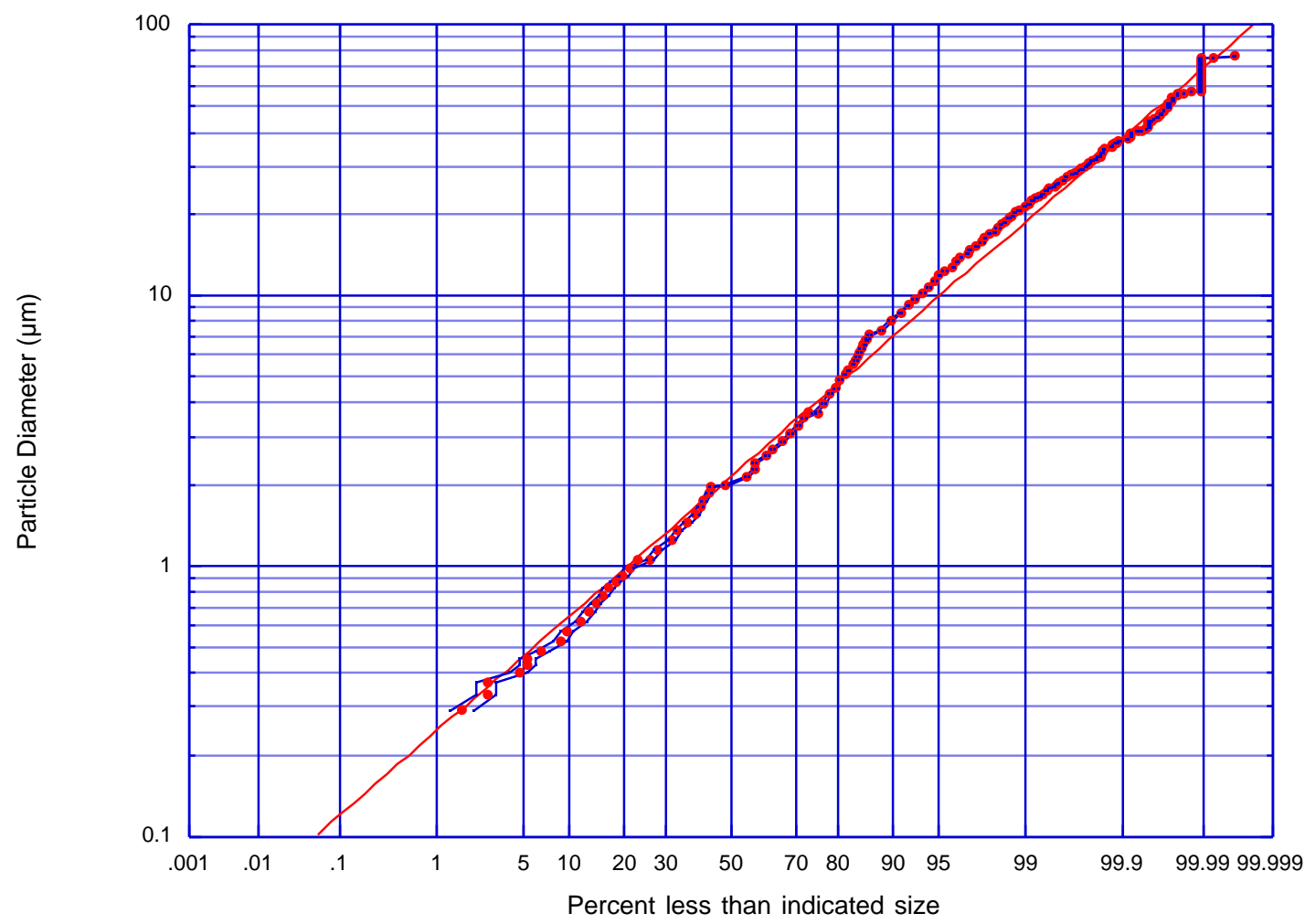

Analysis Summary Table:

\begin{tabular}{|c|c|c|c|c|c|}
\hline \multicolumn{2}{|c|}{$\begin{array}{c}\text { magnification, } \\
\text { number of images }\end{array}$} & \multicolumn{2}{|c|}{ area analyzed $^{1}(\%)$} & counted size range $(\mu \mathrm{m})$ & $\begin{array}{c}\text { total number of } \\
\text { particles (not scaled) }\end{array}$ \\
\hline $50 x$ & 6 & \multicolumn{2}{|r|}{0.894} & $d_{p}>7.31$ & 5341 \\
\hline $100 x$ & 6 & \multicolumn{2}{|r|}{0.224} & $3.88<\mathrm{d}_{\mathrm{p}}<7.31$ & 3463 \\
\hline $200 x$ & 6 & \multicolumn{2}{|r|}{0.0558} & $2.03<d_{p}<3.88$ & 3458 \\
\hline $500 x$ & 6 & \multicolumn{2}{|r|}{0.00893} & $1.18<\mathrm{d}_{\mathrm{p}}<2.03$ & 1321 \\
\hline 1000x, & 6 & \multicolumn{2}{|r|}{0.00217} & $0.00<\mathrm{d}_{\mathrm{p}}<1.18$ & 526 \\
\hline \multicolumn{2}{|c|}{ Totals: } & \multicolumn{2}{|r|}{$1.18 \%$} & - & 14109 \\
\hline \multicolumn{4}{|c|}{ data moments ${ }^{2}:$} & \multicolumn{2}{|c|}{ log-normal fit moments ${ }^{2}$ : } \\
\hline \multicolumn{3}{|c|}{ GMD $(\mu \mathrm{m}):$} & 2.16 & GMD $(\mu \mathrm{m}):$ & 2.16 \\
\hline \multicolumn{2}{|c|}{ GSD: } & & 2.65 & GSD: & 2.54 \\
\hline \multicolumn{3}{|c|}{ lower $95 \%(\mu \mathrm{m})$ : } & 0.31 & $\mathrm{R}^{2}$ & 0.99625 \\
\hline \multirow{2}{*}{\multicolumn{3}{|c|}{ upper $95 \%(\mu \mathrm{m})$ : }} & 15.11 & lower $95 \%(\mu \mathrm{m})$ : & 0.33 \\
\hline & & & & upper $95 \%(\mu \mathrm{m})$ : & 13.88 \\
\hline
\end{tabular}

1. based on overall filter area of $1.59 \times 10^{9} \mu \mathrm{m}^{2}$

2. $\mathrm{GMD}=$ geometric mean diameter, $\mathrm{GSD}=$ geometric standard deviation, $\mathrm{R}=$ linear correlation coefficient

Figure 3.9 Module I, Sample 7 (TSFH07-01) count-based particle size distribution. 


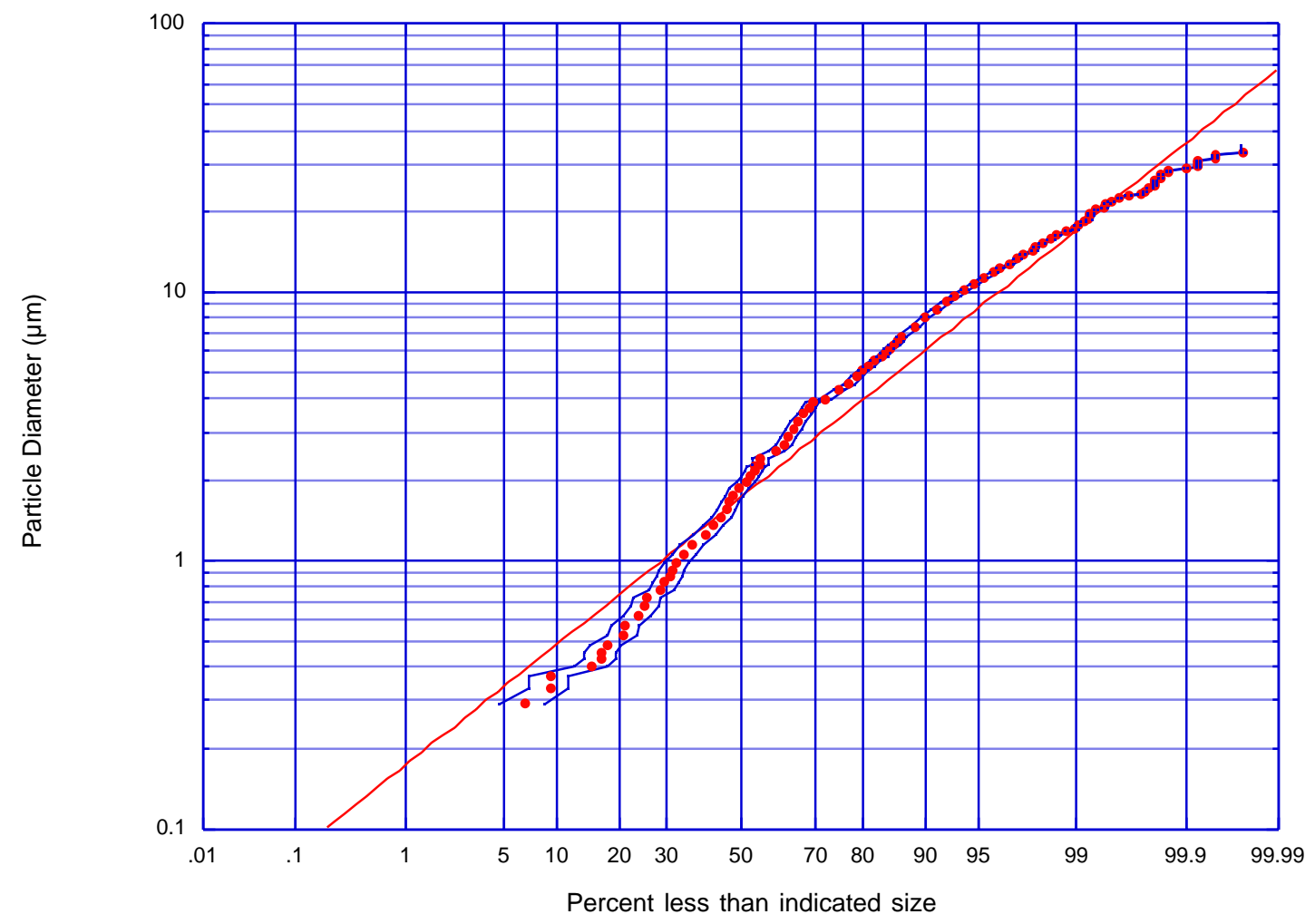

Analysis Summary Table:

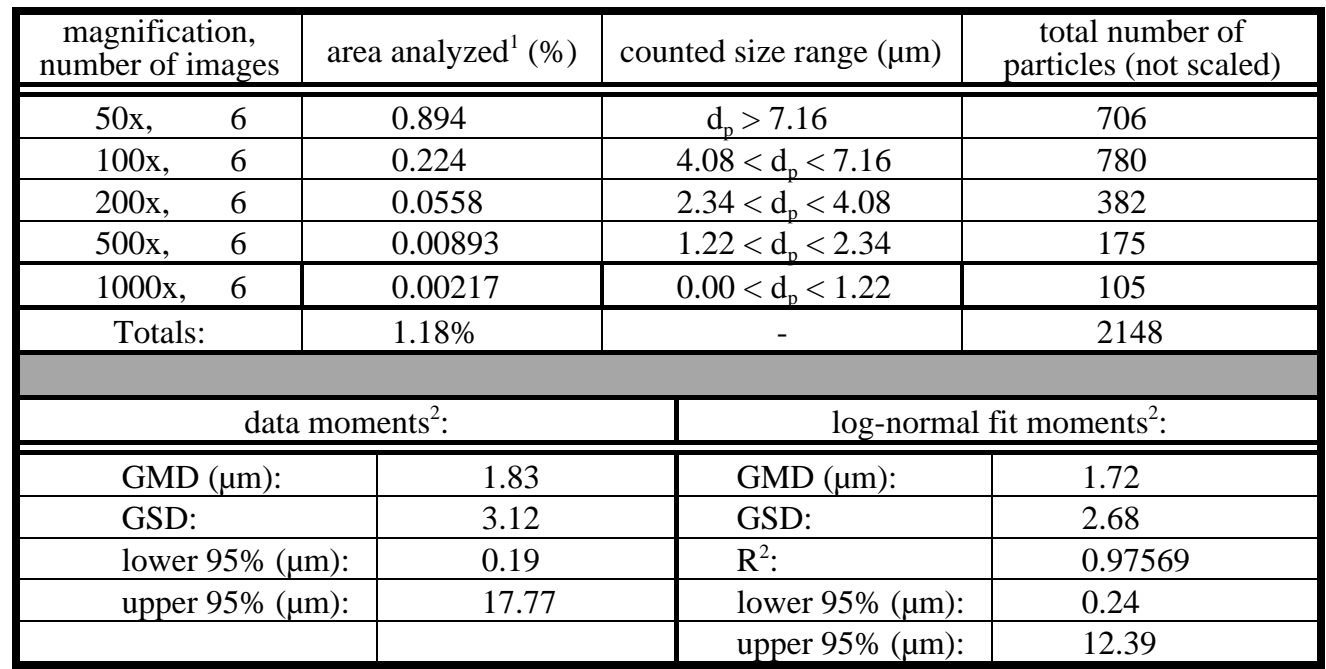

1. based on overall filter area of $1.59 \times 10^{9} \mu \mathrm{m}^{2}$

2. $\mathrm{GMD}=$ geometric mean diameter, $\mathrm{GSD}=$ geometric standard deviation, $\mathrm{R}=$ linear correlation coefficient

Figure 3.10 Module I, Sample 8 (TSFH06-01) count-based particle size distribution. 


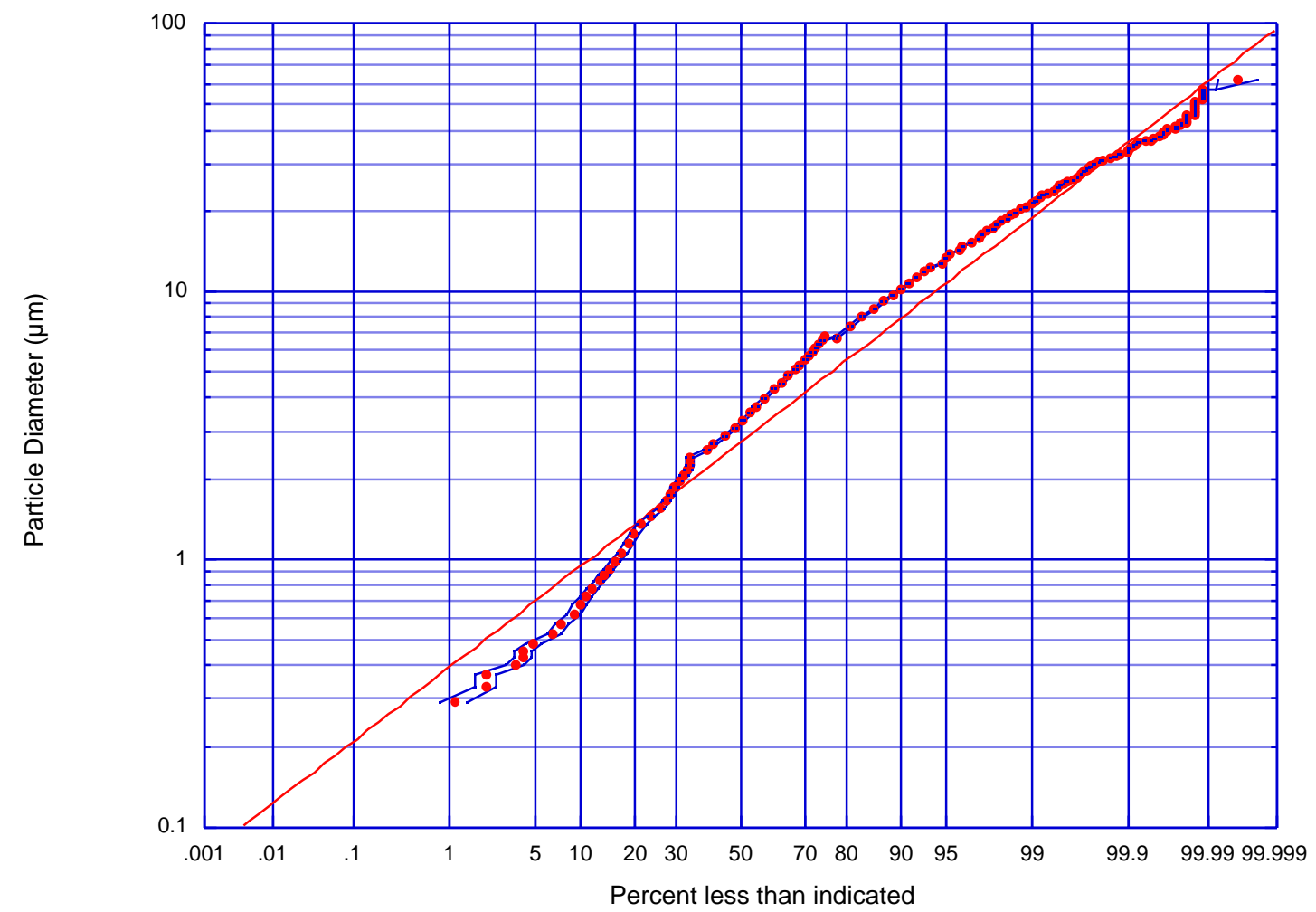

Analysis Summary Table:

\begin{tabular}{|c|c|c|c|c|c|}
\hline \multicolumn{2}{|c|}{$\begin{array}{c}\text { magnification, } \\
\text { number of images }\end{array}$} & \multicolumn{2}{|c|}{ area analyzed $^{1}(\%)$} & counted size range $(\mu \mathrm{m})$ & $\begin{array}{c}\text { total number of } \\
\text { particles (not scaled) }\end{array}$ \\
\hline $50 x$ & 6 & \multicolumn{2}{|r|}{0.894} & $d_{p}>7.06$ & 8174 \\
\hline $100 x$ & 6 & \multicolumn{2}{|r|}{0.224} & $3.95<d_{p}<7.06$ & 5837 \\
\hline $200 x$ & 6 & \multicolumn{2}{|r|}{0.0558} & $2.30<d_{p}<3.95$ & 3936 \\
\hline $500 x$ & 6 & \multicolumn{2}{|r|}{0.00893} & $1.34<\mathrm{d}_{\mathrm{p}}<2.30$ & 1262 \\
\hline 1000x, & 6 & \multicolumn{2}{|r|}{0.00217} & $0.00<\mathrm{d}_{\mathrm{p}}<1.34$ & 396 \\
\hline \multicolumn{2}{|c|}{ Totals: } & \multicolumn{2}{|r|}{$1.18 \%$} & - & 19605 \\
\hline \multicolumn{4}{|c|}{ data moments ${ }^{2}:$} & \multicolumn{2}{|c|}{ log-normal fit moments ${ }^{2}$ : } \\
\hline \multicolumn{3}{|c|}{ GMD $(\mu \mathrm{m}):$} & 3.10 & GMD $(\mu \mathrm{m}):$ & 2.74 \\
\hline \multicolumn{2}{|c|}{ GSD: } & & 2.72 & GSD: & 2.30 \\
\hline \multicolumn{3}{|c|}{ lower $95 \%(\mu \mathrm{m})$ : } & 0.42 & $\mathrm{R}^{2}$ & 0.98351 \\
\hline \multirow{2}{*}{\multicolumn{3}{|c|}{ upper $95 \%(\mu \mathrm{m})$ : }} & 22.93 & lower $95 \%(\mu \mathrm{m})$ : & 0.52 \\
\hline & & & & upper $95 \%(\mu \mathrm{m})$ : & 14.51 \\
\hline
\end{tabular}

1. based on overall filter area of $1.59 \times 10^{9} \mu \mathrm{m}^{2}$

2. $\mathrm{GMD}=$ geometric mean diameter, $\mathrm{GSD}=$ geometric standard deviation, $\mathrm{R}=$ linear correlation coefficient

Figure 3.11 Module I, Sample 9 (TSFH04-01) count-based particle size distribution. 


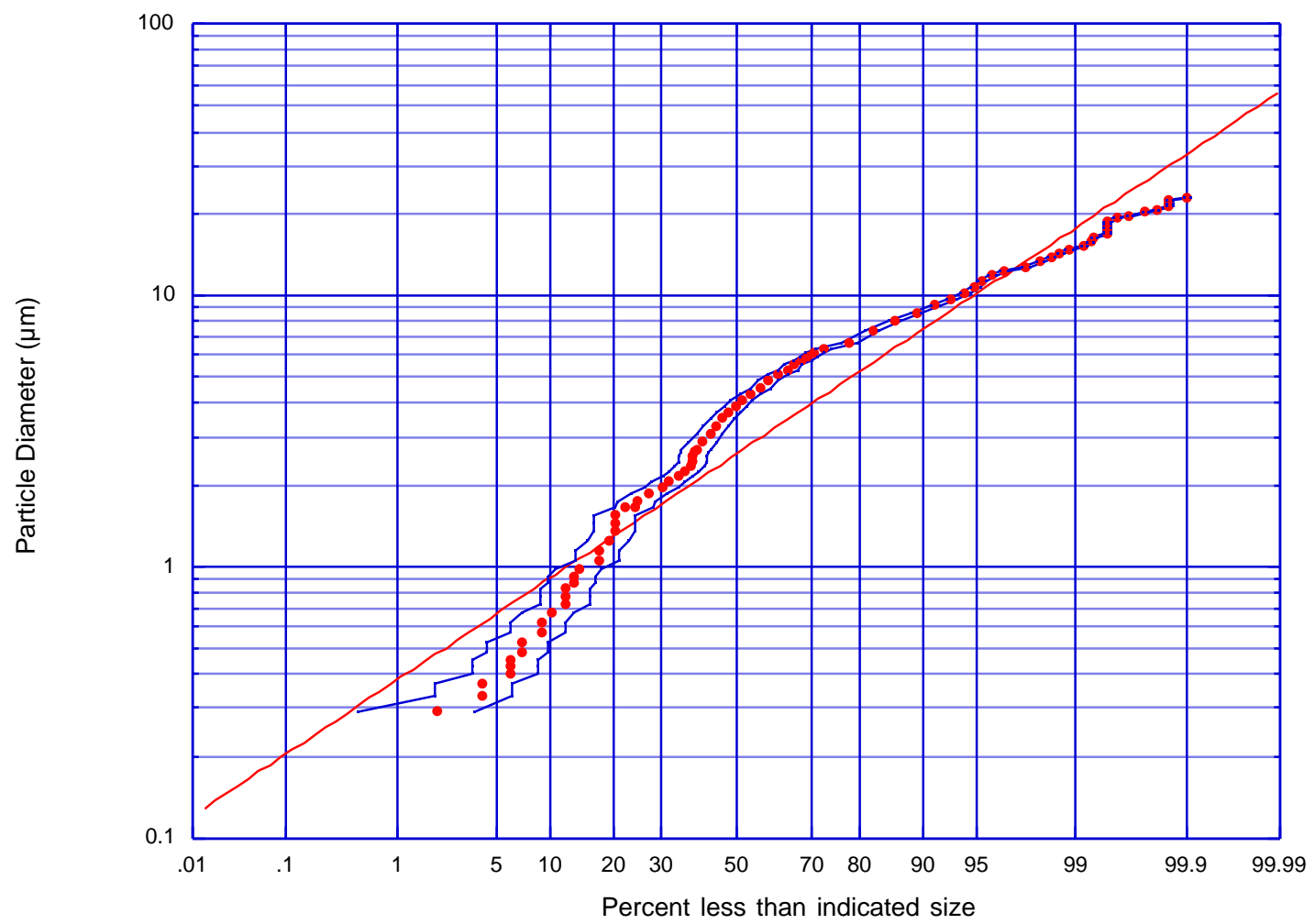

Analysis Summary Table:

\begin{tabular}{|c|c|c|c|c|c|}
\hline \multicolumn{2}{|c|}{$\begin{array}{c}\text { magnification, } \\
\text { number of images }\end{array}$} & \multicolumn{2}{|c|}{ area analyzed $^{1}(\%)$} & counted size range $(\mu \mathrm{m})$ & $\begin{array}{c}\text { total number of } \\
\text { particles (not scaled) }\end{array}$ \\
\hline $50 x$ & 6 & \multicolumn{2}{|r|}{0.894} & $d_{p}>6.64$ & 709 \\
\hline $100 x$ & 6 & \multicolumn{2}{|r|}{0.224} & $4.45<d_{p}<6.64$ & 469 \\
\hline $200 x$ & 6 & \multicolumn{2}{|r|}{0.0558} & $2.76<\mathrm{d}_{\mathrm{p}}<4.45$ & 236 \\
\hline $500 x$ & 6 & \multicolumn{2}{|r|}{0.00893} & $1.77<d_{p}<2.76$ & 155 \\
\hline 1000x, & 6 & \multicolumn{2}{|r|}{0.00217} & $0.00<\mathrm{d}_{\mathrm{p}}<1.77$ & 51 \\
\hline \multicolumn{2}{|c|}{ Totals: } & \multicolumn{2}{|r|}{$1.18 \%$} & - & 1620 \\
\hline \multicolumn{4}{|c|}{ data moments ${ }^{2}$ : } & \multicolumn{2}{|c|}{ log-normal fit moments ${ }^{2}$ : } \\
\hline \multicolumn{3}{|c|}{ GMD $(\mu \mathrm{m}):$} & 3.13 & GMD $(\mu \mathrm{m}):$ & 2.63 \\
\hline \multicolumn{2}{|c|}{ GSD: } & & 2.67 & GSD: & 2.30 \\
\hline \multicolumn{3}{|c|}{ lower $95 \%(\mu \mathrm{m})$ : } & 0.44 & $\mathrm{R}^{2}$ & 0.94518 \\
\hline \multirow{2}{*}{\multicolumn{3}{|c|}{ upper $95 \%(\mu \mathrm{m})$ : }} & 22.41 & lower $95 \%(\mu \mathrm{m})$ : & 0.50 \\
\hline & & & & upper $95 \%(\mu \mathrm{m})$ : & 13.86 \\
\hline
\end{tabular}

1. based on overall filter area of $1.59 \times 10^{9} \mu \mathrm{m}^{2}$

2. $\mathrm{GMD}=$ geometric mean diameter, $\mathrm{GSD}=$ geometric standard deviation, $\mathrm{R}=$ linear correlation coefficient

Figure 3.12 Module I, Sample 10 (TSFH02-01) count-based particle size distribution. 


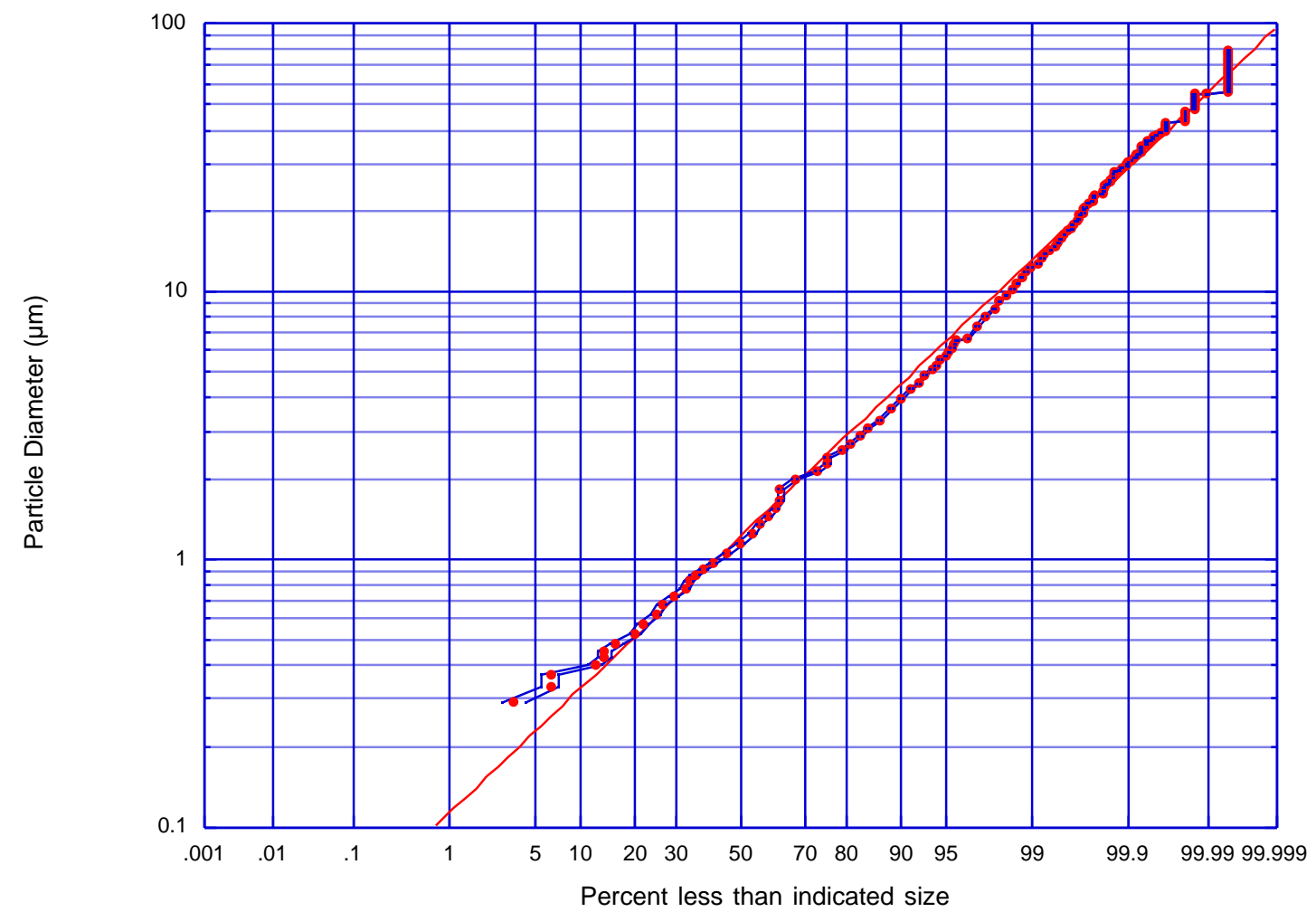

Analysis Summary Table:

\begin{tabular}{|c|c|c|c|c|c|}
\hline \multicolumn{2}{|c|}{$\begin{array}{c}\text { magnification, } \\
\text { number of images }\end{array}$} & \multicolumn{2}{|c|}{ area analyzed $^{1}(\%)$} & counted size range $(\mu \mathrm{m})$ & $\begin{array}{c}\text { total number of } \\
\text { particles (not scaled) }\end{array}$ \\
\hline $50 x$ & 6 & \multicolumn{2}{|r|}{0.894} & $d_{p}>6.61$ & 1096 \\
\hline $100 x$ & 6 & \multicolumn{2}{|r|}{0.224} & $3.32<d_{p}<6.61$ & 1714 \\
\hline $200 x$ & 6 & \multicolumn{2}{|r|}{0.0558} & $1.73<d_{p}<3.32$ & 1979 \\
\hline $500 x$ & 6 & \multicolumn{2}{|r|}{0.00893} & $0.92<d_{p}<1.73$ & 1186 \\
\hline 1000x, & 6 & \multicolumn{2}{|r|}{0.00217} & $0.00<\mathrm{d}_{\mathrm{p}}<0.92$ & 503 \\
\hline \multicolumn{2}{|c|}{ Totals: } & \multicolumn{2}{|r|}{$1.18 \%$} & - & 6478 \\
\hline \multicolumn{4}{|c|}{ data moments ${ }^{2}:$} & \multicolumn{2}{|c|}{ log-normal fit moments ${ }^{2}$ : } \\
\hline \multicolumn{3}{|c|}{ GMD $(\mu \mathrm{m}):$} & 1.30 & GMD $(\mu \mathrm{m}):$ & 1.22 \\
\hline \multicolumn{2}{|c|}{ GSD: } & & 2.43 & GSD: & 2.79 \\
\hline \multicolumn{3}{|c|}{ lower $95 \%(\mu \mathrm{m})$ : } & 0.22 & $\mathrm{R}^{2}$ & 0.99651 \\
\hline \multirow{2}{*}{\multicolumn{3}{|c|}{ upper $95 \%(\mu \mathrm{m})$ : }} & 7.68 & lower $95 \%(\mu \mathrm{m})$ : & 0.16 \\
\hline & & & & upper $95 \%(\mu \mathrm{m})$ : & 9.49 \\
\hline
\end{tabular}

1. based on overall filter area of $1.59 \times 10^{9} \mu \mathrm{m}^{2}$

2. $\mathrm{GMD}=$ geometric mean diameter, $\mathrm{GSD}=$ geometric standard deviation, $\mathrm{R}=$ linear correlation coefficient

Figure 3.13 Module I, Sample 10A (TSFH21-01) count-based particle size distribution. 


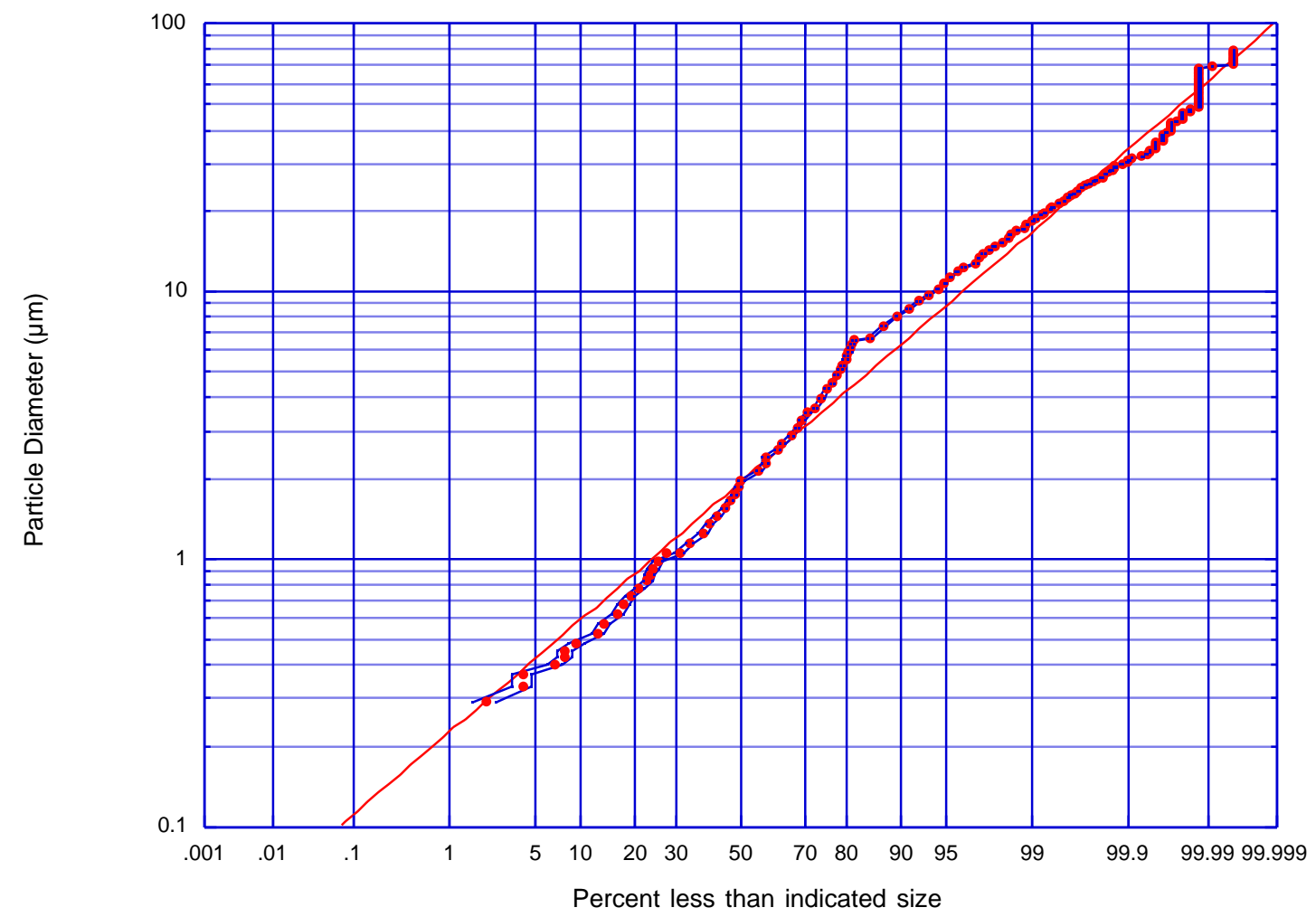

Analysis Summary Table:

\begin{tabular}{|c|c|c|c|c|c|}
\hline \multicolumn{2}{|c|}{$\begin{array}{c}\text { magnification, } \\
\text { number of images }\end{array}$} & \multicolumn{2}{|c|}{ area analyzed $^{1}(\%)$} & counted size range $(\mu \mathrm{m})$ & $\begin{array}{c}\text { total number of } \\
\text { particles (not scaled) }\end{array}$ \\
\hline $50 x$ & 6 & \multicolumn{2}{|r|}{0.894} & $d_{p}>6.75$ & 5248 \\
\hline $100 x$ & 6 & \multicolumn{2}{|r|}{0.224} & $3.70<d_{p}<6.75$ & 2512 \\
\hline $200 x$ & 6 & \multicolumn{2}{|r|}{0.0558} & $1.99<\mathrm{d}_{\mathrm{p}}<3.70$ & 2406 \\
\hline $500 x$ & 6 & \multicolumn{2}{|r|}{0.00893} & $1.13<\mathrm{d}_{\mathrm{p}}<1.99$ & 1233 \\
\hline 1000x, & 6 & \multicolumn{2}{|r|}{0.00217} & $0.00<\mathrm{d}_{\mathrm{p}}<1.13$ & 480 \\
\hline \multicolumn{2}{|c|}{ Totals: } & \multicolumn{2}{|r|}{$1.18 \%$} & - & 11879 \\
\hline \multicolumn{4}{|c|}{ data moments ${ }^{2}:$} & \multicolumn{2}{|c|}{ log-normal fit moments ${ }^{2}$ : } \\
\hline \multicolumn{3}{|c|}{ GMD $(\mu \mathrm{m}):$} & 2.02 & GMD $(\mu \mathrm{m}):$ & 1.94 \\
\hline \multicolumn{2}{|c|}{ GSD: } & & 2.83 & GSD: & 2.52 \\
\hline \multicolumn{3}{|c|}{ lower $95 \%(\mu \mathrm{m})$ : } & 0.25 & $\mathrm{R}^{2}$ & 0.99217 \\
\hline \multirow{2}{*}{\multicolumn{3}{|c|}{ upper $95 \%(\mu \mathrm{m})$ : }} & 16.14 & lower $95 \%(\mu \mathrm{m})$ : & 0.30 \\
\hline & & & & upper $95 \%(\mu \mathrm{m})$ : & 12.39 \\
\hline
\end{tabular}

1. based on overall filter area of $1.59 \times 10^{9} \mu \mathrm{m}^{2}$

2. $\mathrm{GMD}=$ geometric mean diameter, $\mathrm{GSD}=$ geometric standard deviation, $\mathrm{R}=$ linear correlation coefficient

Figure 3.14 Module I, Sample 10B (TSFH03-01) count-based particle size distribution. 


\subsubsection{Module II Results}

Figure 3.15 through Figure 3.26 display details of particle size distributions for corresponding collection locations of Module II. Table 3.2 gives a summary of Module II results.

Table 3.2 Summary of dust collected from locations in Tore Supra Module II.

\begin{tabular}{|c|c|c|c|c|c|c|}
\hline \multirow{2}{*}{$\begin{array}{c}\text { Poloidal } \\
\text { Location }\end{array}$} & Sample & Filter ID & $\begin{array}{c}\text { Sampled } \\
\text { Area }\left(\mathrm{cm}^{2}\right)\end{array}$ & $\begin{array}{c}\text { Collected } \\
\text { Mass }(\mathrm{mg})\end{array}$ & \multicolumn{2}{|c|}{$\begin{array}{c}\text { Count-based Size } \\
\text { Distribution Parameters }\end{array}$} \\
\hline \hline 1 & & & GMD $(\mu \mathrm{m})$ & GSD \\
\hline 2 & 11 & TSFH09-01 & 621.2 & 1.1 & 2.43 & 2.44 \\
\hline 4 & 13 & TSFH18-01 & 1,553 & 2.4 & 1.87 & 2.87 \\
\hline 3 & 14 & TSFH08-01 & 689.0 & 2.7 & 2.50 & 2.55 \\
\hline 6 & 15 & TSFH19-01 & 1,800 & 0.5 & 3.22 & 2.69 \\
\hline 5 & 16 & TSFH17-01 & 658.0 & 0.0 & 2.34 & 3.25 \\
\hline 7 & 17 & TSFH14-01 & 1,200 & 0.4 & 2.92 & 2.64 \\
\hline 8 & 18 & TSFH11-01 & 428.0 & 1.1 & 3.96 & 2.76 \\
\hline 10 & 19 & TSFH10-01 & 1,429 & 0.0 & 2.75 & 3.09 \\
\hline 9 & 20 & TSFH13-01 & 294.0 & 0.8 & 3.19 & 2.61 \\
\hline 11 & $20 \mathrm{~A}$ & TSFH15-01 & 160.0 & 3.5 & 3.13 & 2.92 \\
\hline 12 & $20 \mathrm{~B}$ & TSFH16-01 & 480.0 & 26.7 & 2.65 & 2.72 \\
\hline
\end{tabular}




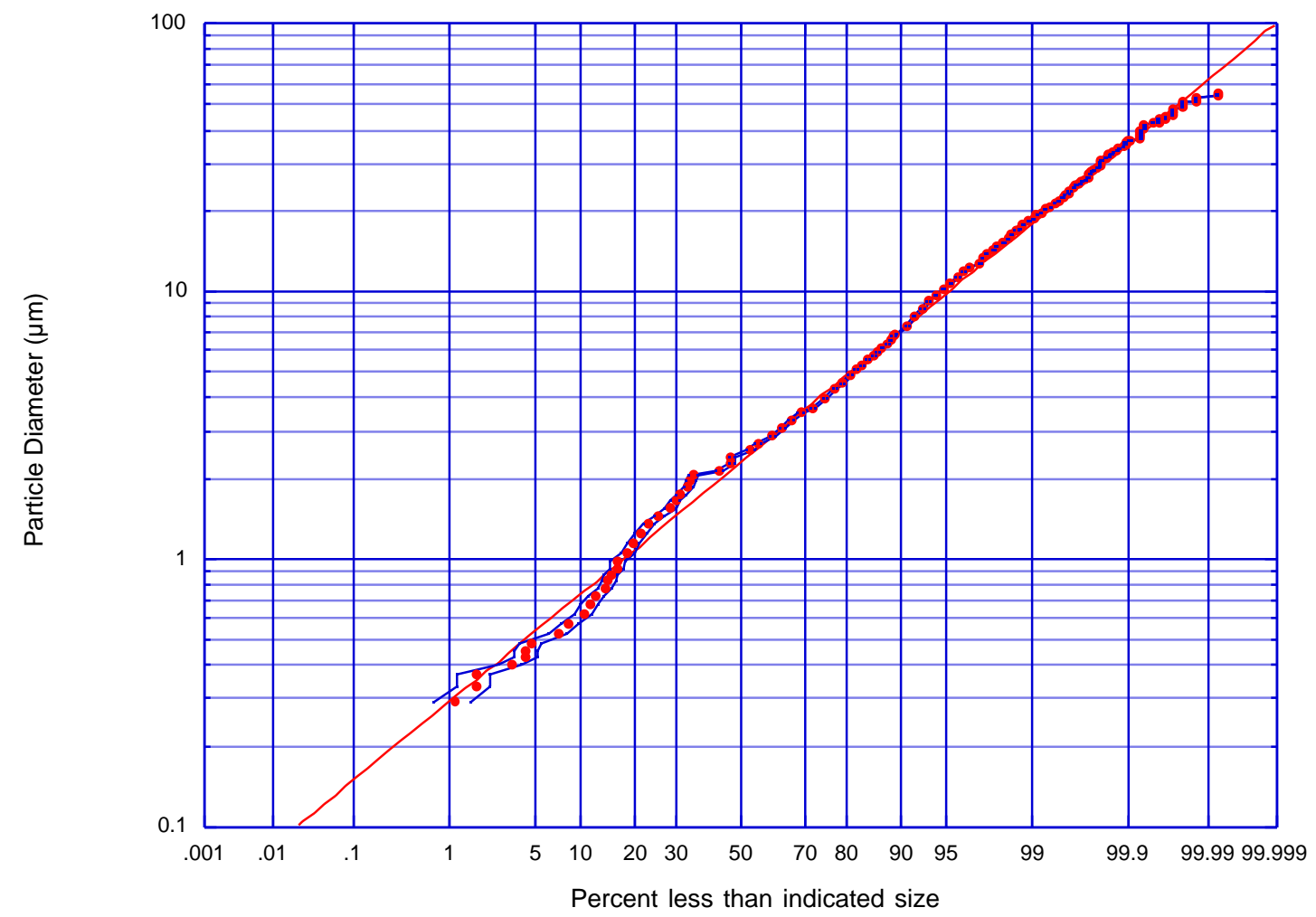

Analysis Summary Table:

\begin{tabular}{|c|c|c|c|c|c|}
\hline \multicolumn{2}{|c|}{$\begin{array}{c}\text { magnification, } \\
\text { number of images }\end{array}$} & \multicolumn{2}{|c|}{ area analyzed $^{1}(\%)$} & counted size range $(\mu \mathrm{m})$ & $\begin{array}{c}\text { total number of } \\
\text { particles (not scaled) }\end{array}$ \\
\hline $50 x$ & 6 & \multicolumn{2}{|r|}{0.894} & $d_{p}>7.12$ & 2080 \\
\hline $100 x$ & 6 & \multicolumn{2}{|r|}{0.224} & $3.78<\mathrm{d}_{\mathrm{p}}<7.12$ & 2597 \\
\hline $200 x$ & 6 & \multicolumn{2}{|r|}{0.0558} & $2.12<d_{p}<3.78$ & 2269 \\
\hline $500 x$ & 6 & \multicolumn{2}{|r|}{0.00893} & $1.32<\mathrm{d}_{\mathrm{p}}<2.12$ & 655 \\
\hline 1000x, & 6 & \multicolumn{2}{|r|}{0.00217} & $0.00<\mathrm{d}_{\mathrm{p}}<1.32$ & 237 \\
\hline \multicolumn{2}{|c|}{ Totals: } & \multicolumn{2}{|r|}{$1.18 \%$} & - & 7838 \\
\hline \multicolumn{4}{|c|}{ data moments ${ }^{2}:$} & \multicolumn{2}{|c|}{ log-normal fit moments ${ }^{2}$ : } \\
\hline \multicolumn{3}{|c|}{ GMD $(\mu \mathrm{m}):$} & 2.43 & GMD $(\mu \mathrm{m}):$ & 230 \\
\hline \multicolumn{2}{|c|}{ GSD: } & & 2.44 & GSD: & 2.42 \\
\hline \multicolumn{3}{|c|}{ lower $95 \%(\mu \mathrm{m})$ : } & 0.41 & $\mathrm{R}^{2}$ & 0.9977 \\
\hline \multirow{2}{*}{\multicolumn{3}{|c|}{ upper $95 \%(\mu \mathrm{m})$ : }} & 14.42 & lower $95 \%(\mu \mathrm{m})$ : & 0.39 \\
\hline & & & & upper $95 \%(\mu \mathrm{m})$ : & 13.49 \\
\hline
\end{tabular}

1. based on overall filter area of $1.59 \times 10^{9} \mu \mathrm{m}^{2}$

2. $\mathrm{GMD}=$ geometric mean diameter, $\mathrm{GSD}=$ geometric standard deviation, $\mathrm{R}=$ linear correlation coefficient

Figure 3.15 Module II, Sample 11 (TSFH09-01) count-based particle size distribution. 


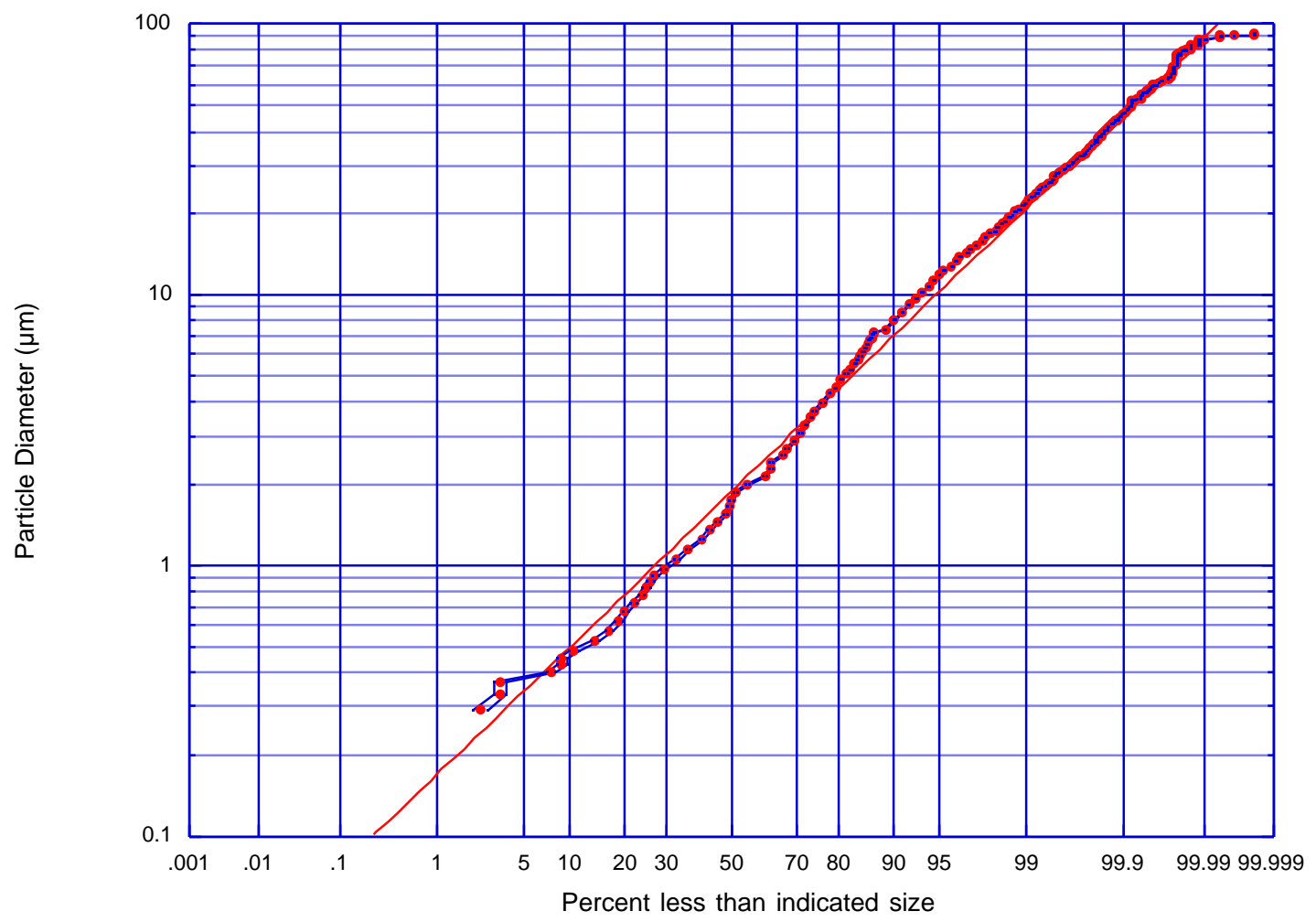

Analysis Summary Table:

\begin{tabular}{|c|c|c|c|c|c|}
\hline \multicolumn{2}{|c|}{$\begin{array}{c}\text { magnification, } \\
\text { number of images }\end{array}$} & \multicolumn{2}{|c|}{ area analyzed $^{1}(\%)$} & counted size range $(\mu \mathrm{m})$ & $\begin{array}{c}\text { total number of } \\
\text { particles (not scaled) }\end{array}$ \\
\hline $50 x$ & 6 & \multicolumn{2}{|r|}{0.894} & $d_{p}>8.36$ & 9096 \\
\hline $100 x$ & 6 & \multicolumn{2}{|r|}{0.224} & $4.41<\mathrm{d}_{\mathrm{p}}<8.36$ & 6873 \\
\hline $200 x$ & 6 & \multicolumn{2}{|r|}{0.0558} & $2.07<\mathrm{d}_{\mathrm{p}}<4.41$ & 5171 \\
\hline $500 x$ & 6 & \multicolumn{2}{|r|}{0.00893} & $1.40<\mathrm{d}_{\mathrm{p}}<2.07$ & 2935 \\
\hline 1000x, & 6 & \multicolumn{2}{|r|}{0.00217} & $0.00<\mathrm{d}_{\mathrm{p}}<1.40$ & 1011 \\
\hline \multicolumn{2}{|c|}{ Totals: } & \multicolumn{2}{|r|}{$1.18 \%$} & - & 25086 \\
\hline \multicolumn{4}{|c|}{ data moments ${ }^{2}:$} & \multicolumn{2}{|c|}{ log-normal fit moments ${ }^{2}$ : } \\
\hline \multicolumn{3}{|c|}{ GMD $(\mu \mathrm{m}):$} & 1.87 & GMD $(\mu \mathrm{m}):$ & 1.88 \\
\hline \multicolumn{2}{|c|}{ GSD: } & & 2.87 & GSD: & 2.82 \\
\hline \multicolumn{3}{|c|}{ lower $95 \%(\mu \mathrm{m})$ : } & 0.23 & $\mathrm{R}^{2}$ & 0.99634 \\
\hline \multirow{2}{*}{\multicolumn{3}{|c|}{ upper $95 \%(\mu \mathrm{m})$ : }} & 15.41 & lower $95 \%(\mu \mathrm{m})$ : & 0.24 \\
\hline & & & & upper $95 \%(\mu \mathrm{m})$ : & 14.95 \\
\hline
\end{tabular}

1. based on overall filter area of $1.59 \times 10^{9} \mu \mathrm{m}^{2}$

2. $\mathrm{GMD}=$ geometric mean diameter, $\mathrm{GSD}=$ geometric standard deviation, $\mathrm{R}=$ linear correlation coefficient

Figure 3.16 Module II, Sample 12 (TSFH18-01) count-based particle size distribution. 


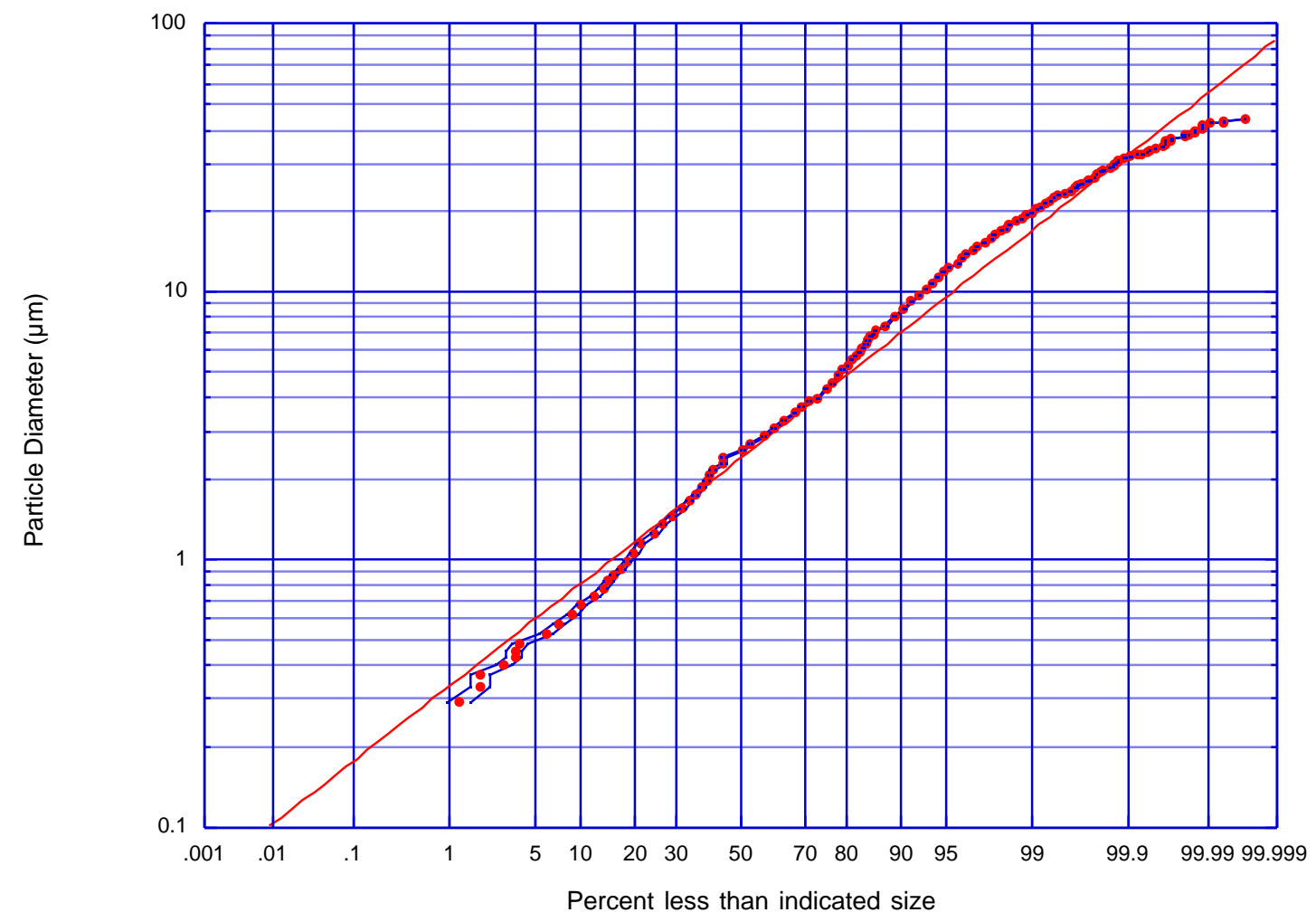

Analysis Summary Table:

\begin{tabular}{|c|c|c|c|c|c|}
\hline \multicolumn{2}{|c|}{$\begin{array}{c}\text { magnification, } \\
\text { number of images }\end{array}$} & \multicolumn{2}{|c|}{ area analyzed $^{1}(\%)$} & counted size range $(\mu \mathrm{m})$ & $\begin{array}{c}\text { total number of } \\
\text { particles (not scaled) }\end{array}$ \\
\hline $50 x$ & 6 & \multicolumn{2}{|r|}{0.894} & $d_{p}>7.32$ & 6520 \\
\hline $100 x$ & 6 & \multicolumn{2}{|r|}{0.224} & $4.03<d_{p}<7.32$ & 5462 \\
\hline $200 x$ & 6 & \multicolumn{2}{|r|}{0.0558} & $2.27<\mathrm{d}_{\mathrm{p}}<4.03$ & 5908 \\
\hline $500 x$ & 6 & \multicolumn{2}{|r|}{0.00893} & $1.38<\mathrm{d}_{\mathrm{p}}<2.27$ & 1766 \\
\hline 1000x, & 6 & \multicolumn{2}{|r|}{0.00217} & $0.00<\mathrm{d}_{\mathrm{p}}<1.38$ & 624 \\
\hline \multicolumn{2}{|c|}{ Totals: } & \multicolumn{2}{|r|}{$1.18 \%$} & - & 20280 \\
\hline \multicolumn{4}{|c|}{ data moments ${ }^{2}:$} & \multicolumn{2}{|c|}{ log-normal fit moments ${ }^{2}$ : } \\
\hline \multicolumn{3}{|c|}{ GMD $(\mu \mathrm{m}):$} & 2.50 & GMD $(\mu \mathrm{m}):$ & 2.39 \\
\hline \multicolumn{2}{|c|}{ GSD: } & & 2.55 & GSD: & 2.32 \\
\hline \multicolumn{3}{|c|}{ lower $95 \%(\mu \mathrm{m})$ : } & 0.38 & $\mathrm{R}^{2}$ & 0.9876 \\
\hline \multirow{2}{*}{\multicolumn{3}{|c|}{ upper $95 \%(\mu \mathrm{m})$ : }} & 16.27 & lower $95 \%(\mu \mathrm{m})$ : & 0.44 \\
\hline & & & & upper $95 \%(\mu \mathrm{m})$ : & 12.93 \\
\hline
\end{tabular}

1. based on overall filter area of $1.59 \times 10^{9} \mu \mathrm{m}^{2}$

2. $\mathrm{GMD}=$ geometric mean diameter, $\mathrm{GSD}=$ geometric standard deviation, $\mathrm{R}=$ linear correlation coefficient

Figure 3.17 Module II, Sample 13 (TSFH08-01) count-based particle size distribution. 


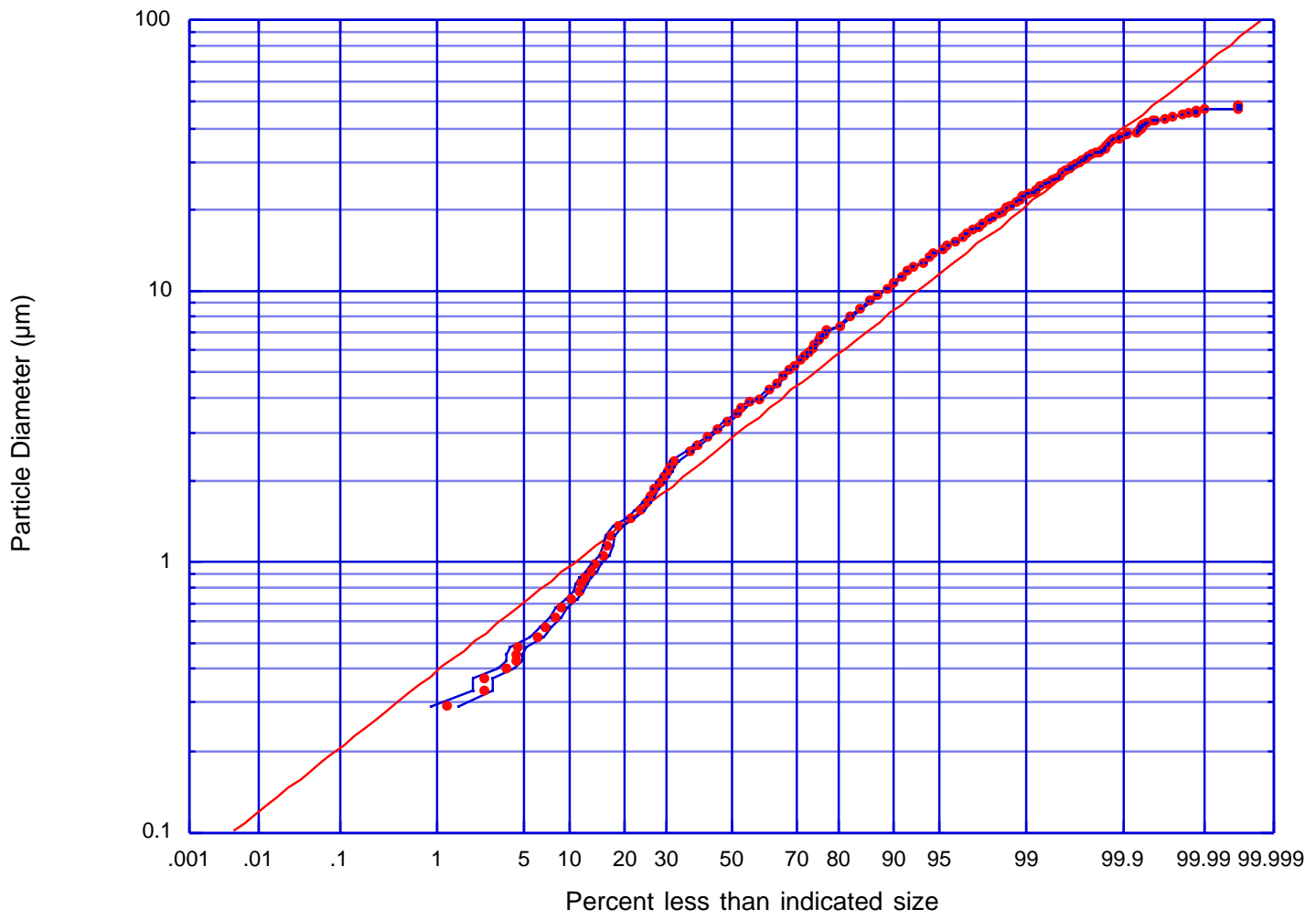

Analysis Summary Table:

\begin{tabular}{|c|c|c|c|c|c|}
\hline \multicolumn{2}{|c|}{$\begin{array}{c}\text { magnification, } \\
\text { number of images }\end{array}$} & \multicolumn{2}{|c|}{ area analyzed $^{1}(\%)$} & counted size range $(\mu \mathrm{m})$ & $\begin{array}{c}\text { total number of } \\
\text { particles (not scaled) }\end{array}$ \\
\hline $50 x$ & 6 & \multicolumn{2}{|r|}{0.894} & $d_{p}>7.32$ & 9551 \\
\hline $100 x$ & 6 & \multicolumn{2}{|r|}{0.224} & $4.13<d_{p}<7.32$ & 7080 \\
\hline $200 x$ & 6 & \multicolumn{2}{|r|}{0.0558} & $2.41<\mathrm{d}_{\mathrm{p}}<4.13$ & 4834 \\
\hline $500 x$ & 6 & \multicolumn{2}{|r|}{0.00893} & $1.36<\mathrm{d}_{\mathrm{p}}<2.41$ & 1398 \\
\hline 1000x, & 6 & \multicolumn{2}{|r|}{0.00217} & $0.00<\mathrm{d}_{\mathrm{p}}<1.36$ & 431 \\
\hline \multicolumn{2}{|c|}{ Totals: } & \multicolumn{2}{|r|}{$1.18 \%$} & - & 23294 \\
\hline \multicolumn{4}{|c|}{ data moments ${ }^{2}:$} & \multicolumn{2}{|c|}{ log-normal fit moments ${ }^{2}$ : } \\
\hline \multicolumn{3}{|c|}{ GMD $(\mu \mathrm{m}):$} & 3.22 & GMD $(\mu \mathrm{m}):$ & 2.85 \\
\hline \multicolumn{2}{|c|}{ GSD: } & & 2.69 & GSD: & 2.35 \\
\hline \multicolumn{3}{|c|}{ lower $95 \%(\mu \mathrm{m})$ : } & 0.44 & $\mathrm{R}^{2}$ & 0.97963 \\
\hline \multirow{2}{*}{\multicolumn{3}{|c|}{ upper $95 \%(\mu \mathrm{m})$ : }} & 23.34 & lower $95 \%(\mu \mathrm{m})$ : & 0.52 \\
\hline & & & & upper $95 \%(\mu \mathrm{m})$ : & 15.70 \\
\hline
\end{tabular}

1. based on overall filter area of $1.59 \times 10^{9} \mu \mathrm{m}^{2}$

2. $\mathrm{GMD}=$ geometric mean diameter, $\mathrm{GSD}=$ geometric standard deviation, $\mathrm{R}=$ linear correlation coefficient

Figure 3.18 Module II, Sample 14 (TSFH19-01) count-based particle size distribution. 


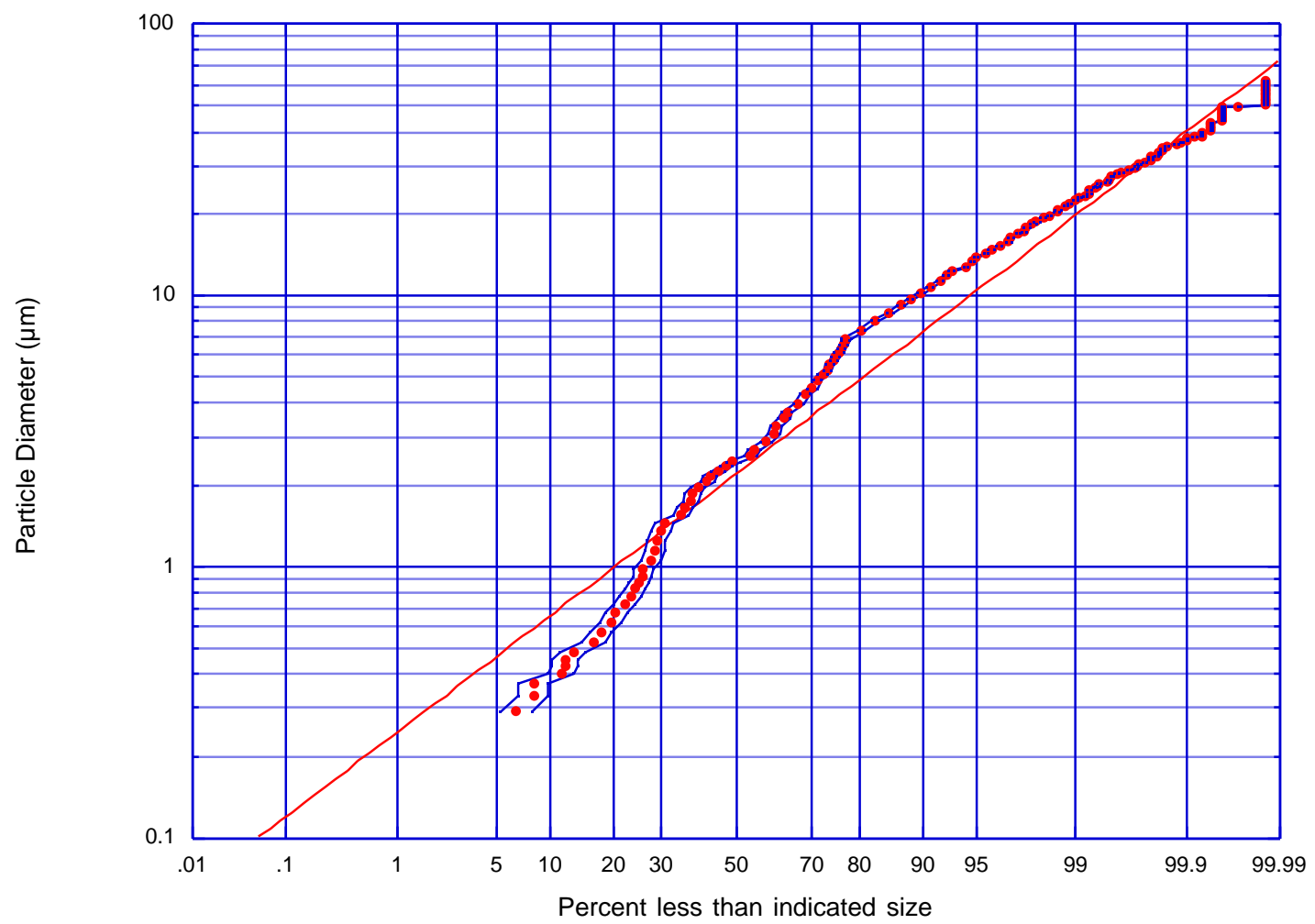

Analysis Summary Table:

\begin{tabular}{|c|c|c|c|c|c|}
\hline \multicolumn{2}{|c|}{$\begin{array}{c}\text { magnification, } \\
\text { number of images }\end{array}$} & \multicolumn{2}{|c|}{ area analyzed $^{1}(\%)$} & counted size range $(\mu \mathrm{m})$ & $\begin{array}{c}\text { total number of } \\
\text { particles (not scaled) }\end{array}$ \\
\hline $50 x$ & 6 & \multicolumn{2}{|r|}{0.894} & $d_{p}>7.14$ & 2149 \\
\hline $100 x$ & 6 & \multicolumn{2}{|r|}{0.224} & $3.92<d_{p}<7.14$ & 999 \\
\hline $200 x$ & 6 & \multicolumn{2}{|r|}{0.0558} & $2.54<d_{p}<3.92$ & 761 \\
\hline $500 x$ & 6 & \multicolumn{2}{|r|}{0.00893} & $1.47<\mathrm{d}_{\mathrm{p}}<2.54$ & 383 \\
\hline 1000x, & 6 & \multicolumn{2}{|r|}{0.00217} & $0.00<\mathrm{d}_{\mathrm{p}}<1.47$ & 141 \\
\hline \multicolumn{2}{|c|}{ Totals: } & \multicolumn{2}{|r|}{$1.18 \%$} & - & 4433 \\
\hline \multicolumn{4}{|c|}{ data moments ${ }^{2}:$} & \multicolumn{2}{|c|}{ log-normal fit moments ${ }^{2}$ : } \\
\hline \multicolumn{3}{|c|}{ GMD $(\mu \mathrm{m}):$} & 2.34 & GMD $(\mu \mathrm{m}):$ & 2.20 \\
\hline \multicolumn{2}{|c|}{ GSD: } & & 3.25 & GSD: & 2.57 \\
\hline \multicolumn{3}{|c|}{ lower $95 \%(\mu \mathrm{m})$ : } & 0.22 & $\mathrm{R}^{2}$ & 0.97613 \\
\hline \multirow{2}{*}{\multicolumn{3}{|c|}{ upper $95 \%(\mu \mathrm{m})$ : }} & 24.79 & lower $95 \%(\mu \mathrm{m})$ : & 0.33 \\
\hline & & & & upper $95 \%(\mu \mathrm{m})$ : & 14.51 \\
\hline
\end{tabular}

1. based on overall filter area of $1.59 \times 10^{9} \mu \mathrm{m}^{2}$

2. $\mathrm{GMD}=$ geometric mean diameter, $\mathrm{GSD}=$ geometric standard deviation, $\mathrm{R}=$ linear correlation coefficient

Figure 3.19 Module II, Sample 15 (TSFH17-01) count-based particle size distribution. 


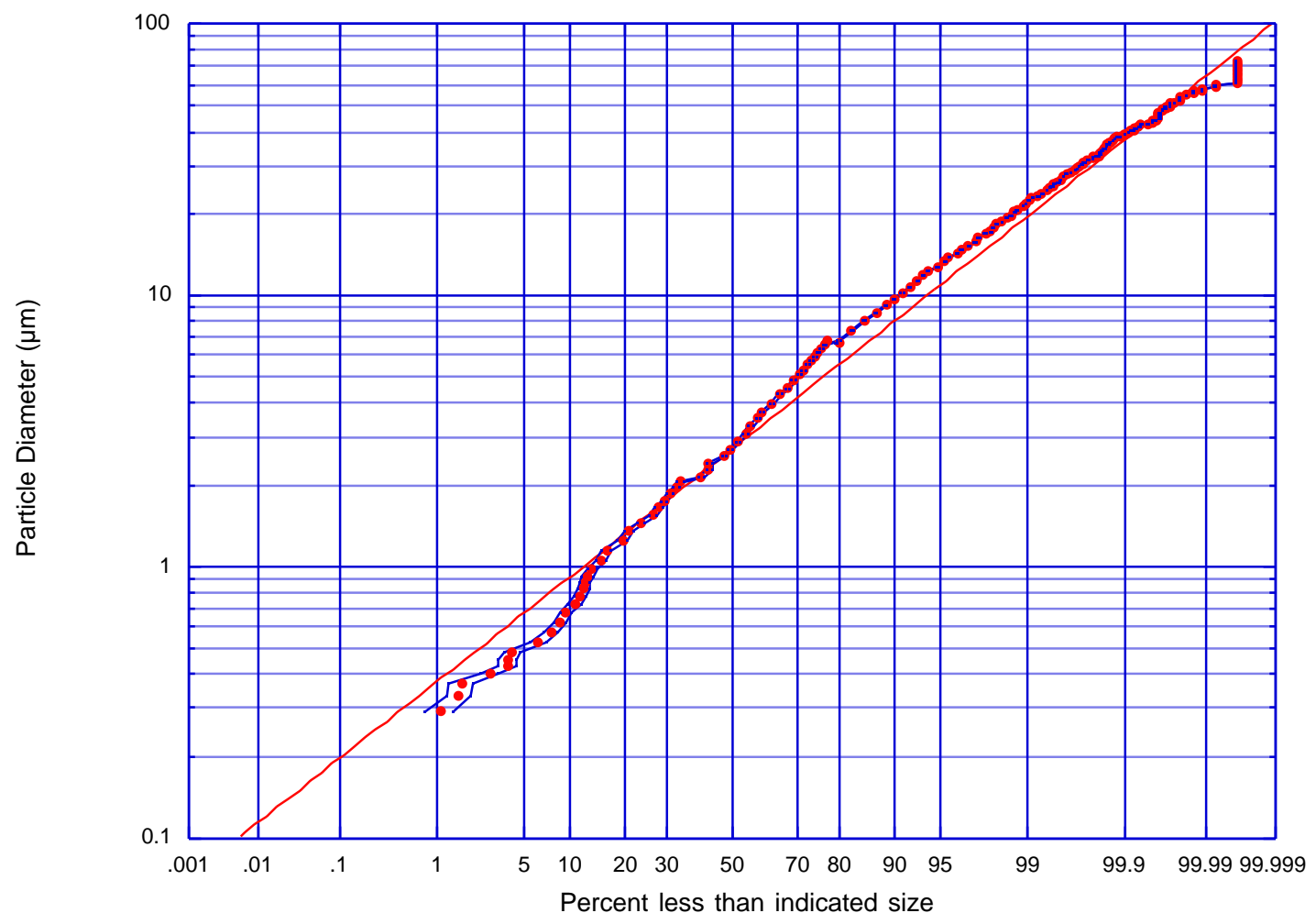

Analysis Summary Table:

\begin{tabular}{|c|c|c|c|c|c|}
\hline \multicolumn{2}{|c|}{$\begin{array}{c}\text { magnification, } \\
\text { number of images }\end{array}$} & \multicolumn{2}{|c|}{ area analyzed $^{1}(\%)$} & counted size range $(\mu \mathrm{m})$ & $\begin{array}{c}\text { total number of } \\
\text { particles (not scaled) }\end{array}$ \\
\hline $50 x$ & 6 & \multicolumn{2}{|r|}{0.894} & $d_{p}>7.13$ & 7772 \\
\hline $100 x$ & 6 & \multicolumn{2}{|r|}{0.224} & $3.91<d_{p}<7.13$ & 5440 \\
\hline $200 x$ & 6 & \multicolumn{2}{|r|}{0.0558} & $2.16<d_{p}<3.91$ & 3463 \\
\hline $500 x$ & 6 & \multicolumn{2}{|r|}{0.00893} & $1.29<\mathrm{d}_{\mathrm{p}}<2.16$ & 1312 \\
\hline 1000x, & 6 & \multicolumn{2}{|r|}{0.00217} & $0.00<\mathrm{d}_{\mathrm{p}}<1.29$ & 376 \\
\hline \multicolumn{2}{|c|}{ Totals: } & \multicolumn{2}{|r|}{$1.18 \%$} & - & 18363 \\
\hline \multicolumn{4}{|c|}{ data moments ${ }^{2}:$} & \multicolumn{2}{|c|}{ log-normal fit moments ${ }^{2}$ : } \\
\hline \multicolumn{3}{|c|}{ GMD $(\mu \mathrm{m}):$} & 2.92 & GMD $(\mu \mathrm{m}):$ & 2.72 \\
\hline \multicolumn{2}{|c|}{ GSD: } & & 2.64 & GSD: & 2.34 \\
\hline \multicolumn{3}{|c|}{ lower $95 \%(\mu \mathrm{m})$ : } & 0.42 & $\mathrm{R}^{2}$ & 0.99096 \\
\hline \multirow{2}{*}{\multicolumn{3}{|c|}{ upper $95 \%(\mu \mathrm{m})$ : }} & 20.34 & lower $95 \%(\mu \mathrm{m})$ : & 0.50 \\
\hline & & & & upper $95 \%(\mu \mathrm{m})$ : & 14.86 \\
\hline
\end{tabular}

1. based on overall filter area of $1.59 \times 10^{9} \mu \mathrm{m}^{2}$

2. $\mathrm{GMD}=$ geometric mean diameter, $\mathrm{GSD}=$ geometric standard deviation, $\mathrm{R}=$ linear correlation coefficient

Figure 3.20 Module II, Sample 16 (TSFH14-01) count-based particle size distribution. 


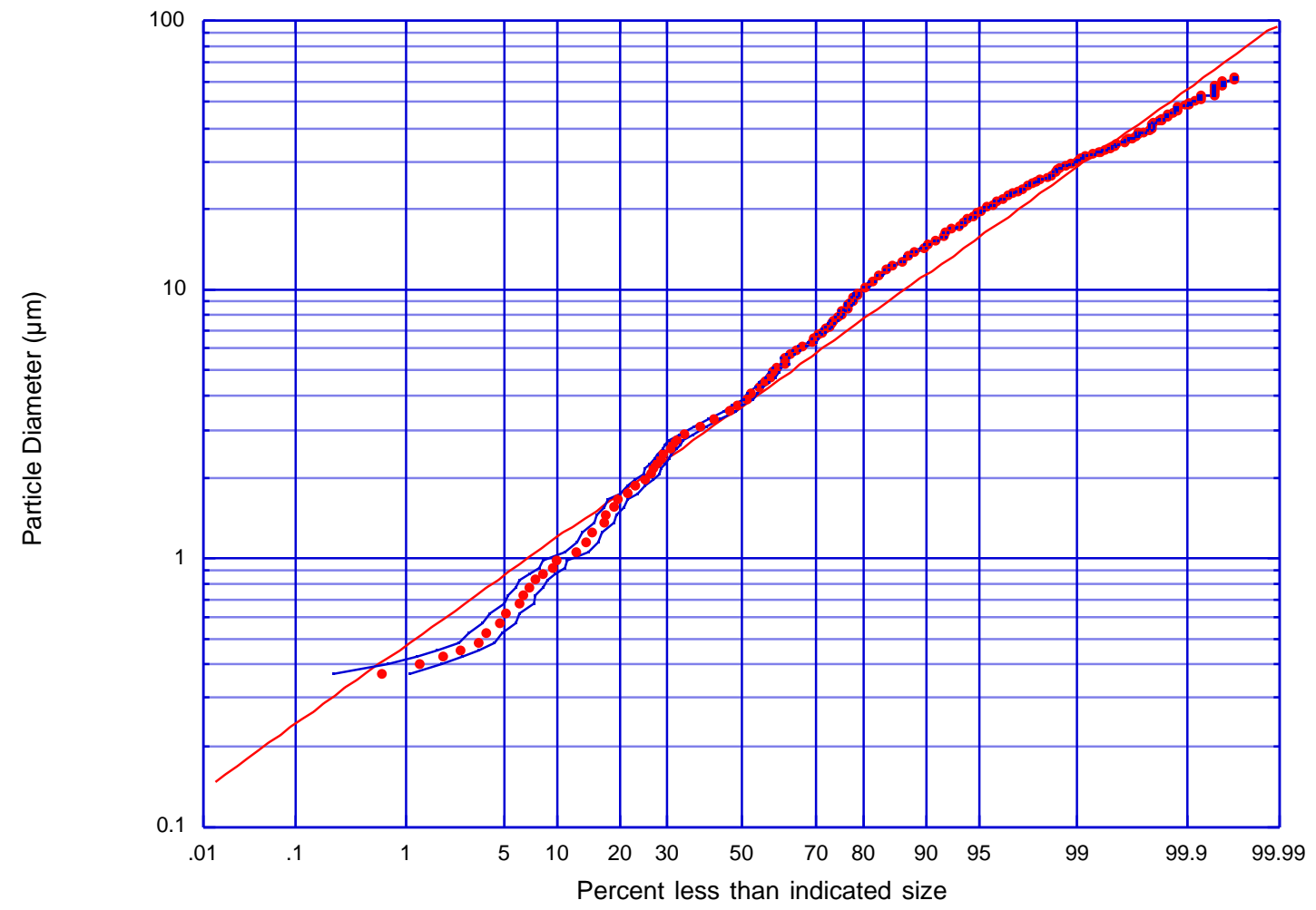

Analysis Summary Table:

\begin{tabular}{|c|c|c|c|c|c|}
\hline \multicolumn{2}{|c|}{$\begin{array}{c}\text { magnification, } \\
\text { number of images }\end{array}$} & \multicolumn{2}{|c|}{ area analyzed $^{1}(\%)$} & counted size range $(\mu \mathrm{m})$ & $\begin{array}{c}\text { total number of } \\
\text { particles (not scaled) }\end{array}$ \\
\hline 50x, & 6 & \multicolumn{2}{|r|}{0.894} & $d_{p}>9.62$ & 3255 \\
\hline $100 x$ & 6 & \multicolumn{2}{|r|}{0.224} & $5.25<d_{p}<9.62$ & 2179 \\
\hline $200 x$ & 6 & \multicolumn{2}{|r|}{0.0558} & $2.78<d_{p}<5.25$ & 1795 \\
\hline $500 x$ & 6 & \multicolumn{2}{|r|}{0.00893} & $1.70<\mathrm{d}_{\mathrm{p}}<2.78$ & 475 \\
\hline 1000x, & 6 & \multicolumn{2}{|r|}{0.00217} & $0.00<\mathrm{d}_{\mathrm{p}}<1.70$ & 144 \\
\hline \multicolumn{2}{|c|}{ Totals: } & \multicolumn{2}{|r|}{$1.18 \%$} & - & 7848 \\
\hline \multicolumn{4}{|c|}{ data moments ${ }^{2}:$} & \multicolumn{2}{|c|}{ log-normal fit moments ${ }^{2}$ : } \\
\hline \multicolumn{3}{|c|}{ GMD $(\mu \mathrm{m}):$} & 3.96 & GMD $(\mu \mathrm{m}):$ & 3.68 \\
\hline \multicolumn{2}{|c|}{ GSD: } & & 2.76 & GSD: & 2.41 \\
\hline \multicolumn{3}{|c|}{ lower $95 \%(\mu \mathrm{m})$ : } & 0.52 & $\mathrm{R}^{2}:$ & 0.98536 \\
\hline \multicolumn{3}{|c|}{ upper $95 \%(\mu \mathrm{m})$ : } & 30.04 & lower $95 \%(\mu \mathrm{m})$ : & 0.63 \\
\hline & & & & upper $95 \%(\mu \mathrm{m})$ : & 21.43 \\
\hline
\end{tabular}

1. based on overall filter area of $1.59 \times 10^{9} \mu \mathrm{m}^{2}$

2. $\mathrm{GMD}=$ geometric mean diameter, $\mathrm{GSD}=$ geometric standard deviation, $\mathrm{R}=$ linear correlation coefficient

Figure 3.21 Module II, Sample 17 (TSFH27-03) count-based particle size distribution. 


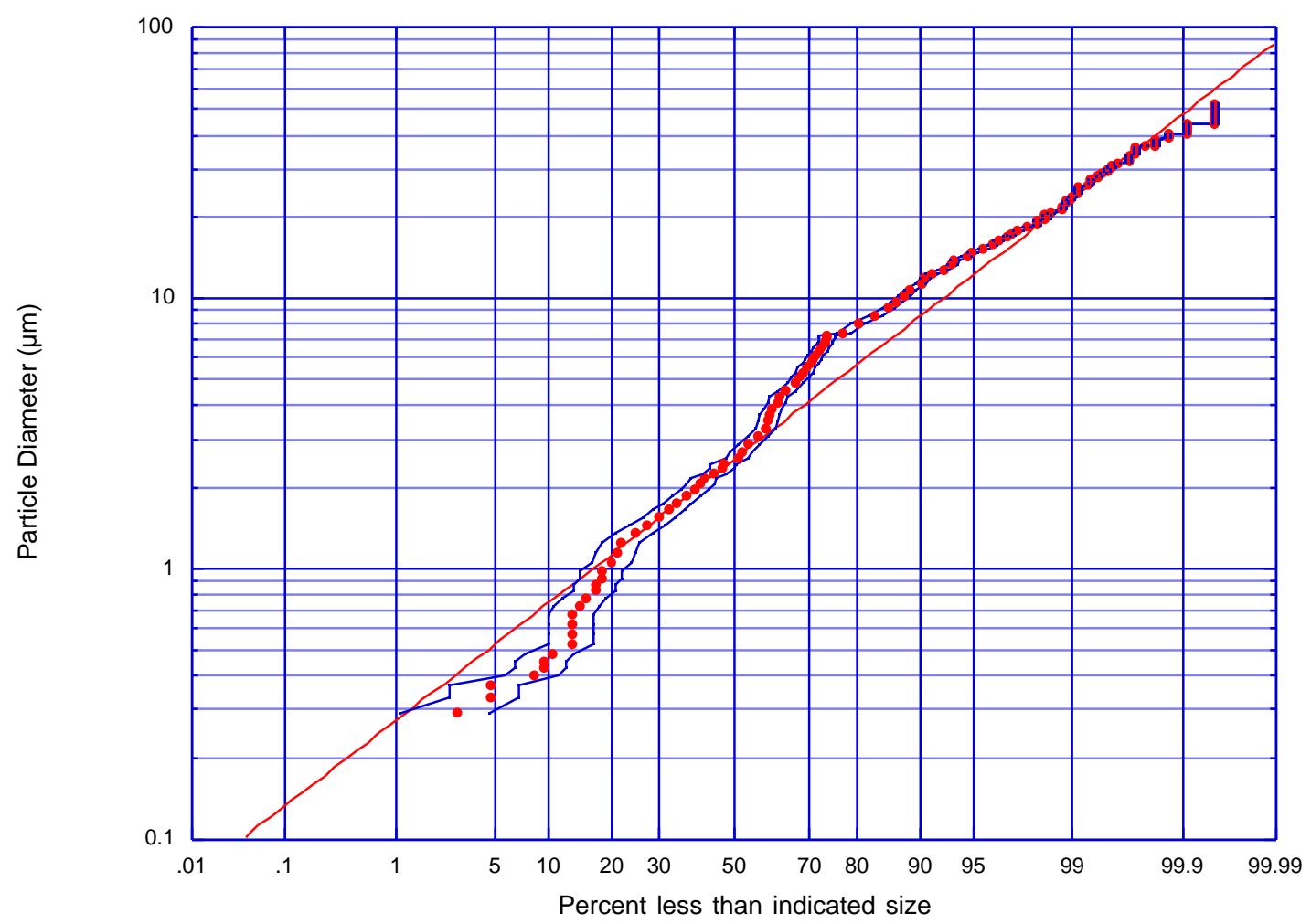

Analysis Summary Table:

\begin{tabular}{|c|c|c|c|c|c|}
\hline \multicolumn{2}{|c|}{$\begin{array}{c}\text { magnification, } \\
\text { number of images }\end{array}$} & \multicolumn{2}{|c|}{ area analyzed $^{1}(\%)$} & counted size range $(\mu \mathrm{m})$ & $\begin{array}{c}\text { total number of } \\
\text { particles (not scaled) }\end{array}$ \\
\hline $50 x$ & 6 & \multicolumn{2}{|r|}{0.894} & $d_{p}>7.46$ & 752 \\
\hline $100 x$ & 6 & \multicolumn{2}{|r|}{0.224} & $4.50<d_{p}<7.46$ & 348 \\
\hline $200 x$ & 6 & \multicolumn{2}{|r|}{0.0558} & $2.57<\mathrm{d}_{\mathrm{p}}<4.50$ & 207 \\
\hline $500 x$ & 6 & \multicolumn{2}{|r|}{0.00893} & $1.42<\mathrm{d}_{\mathrm{p}}<2.57$ & 123 \\
\hline 1000x, & 6 & \multicolumn{2}{|r|}{0.00217} & $0.00<\mathrm{d}_{\mathrm{p}}<1.42$ & 46 \\
\hline \multicolumn{2}{|c|}{ Totals: } & \multicolumn{2}{|r|}{$1.18 \%$} & - & \\
\hline \multicolumn{4}{|c|}{ data moments ${ }^{2}:$} & \multicolumn{2}{|c|}{ log-normal fit moments ${ }^{2}$ : } \\
\hline \multicolumn{3}{|c|}{ GMD $(\mu \mathrm{m}):$} & 2.75 & GMD $(\mu \mathrm{m}):$ & 2.53 \\
\hline \multicolumn{2}{|c|}{ GSD: } & & 3.09 & GSD: & 2.60 \\
\hline \multicolumn{3}{|c|}{ lower $95 \%(\mu \mathrm{m})$ : } & 0.29 & $\mathrm{R}^{2}$ & 0.98002 \\
\hline \multirow{2}{*}{\multicolumn{3}{|c|}{ upper $95 \%(\mu \mathrm{m})$ : }} & 26.20 & lower $95 \%(\mu \mathrm{m})$ : & 0.38 \\
\hline & & & & upper $95 \%(\mu \mathrm{m})$ : & 17.07 \\
\hline
\end{tabular}

1. based on overall filter area of $1.59 \times 10^{9} \mu \mathrm{m}^{2}$

2. $\mathrm{GMD}=$ geometric mean diameter, $\mathrm{GSD}=$ geometric standard deviation, $\mathrm{R}=$ linear correlation coefficient

Figure 3.22 Module II, Sample 18 (TSFH11-01) count-based particle size distribution. 


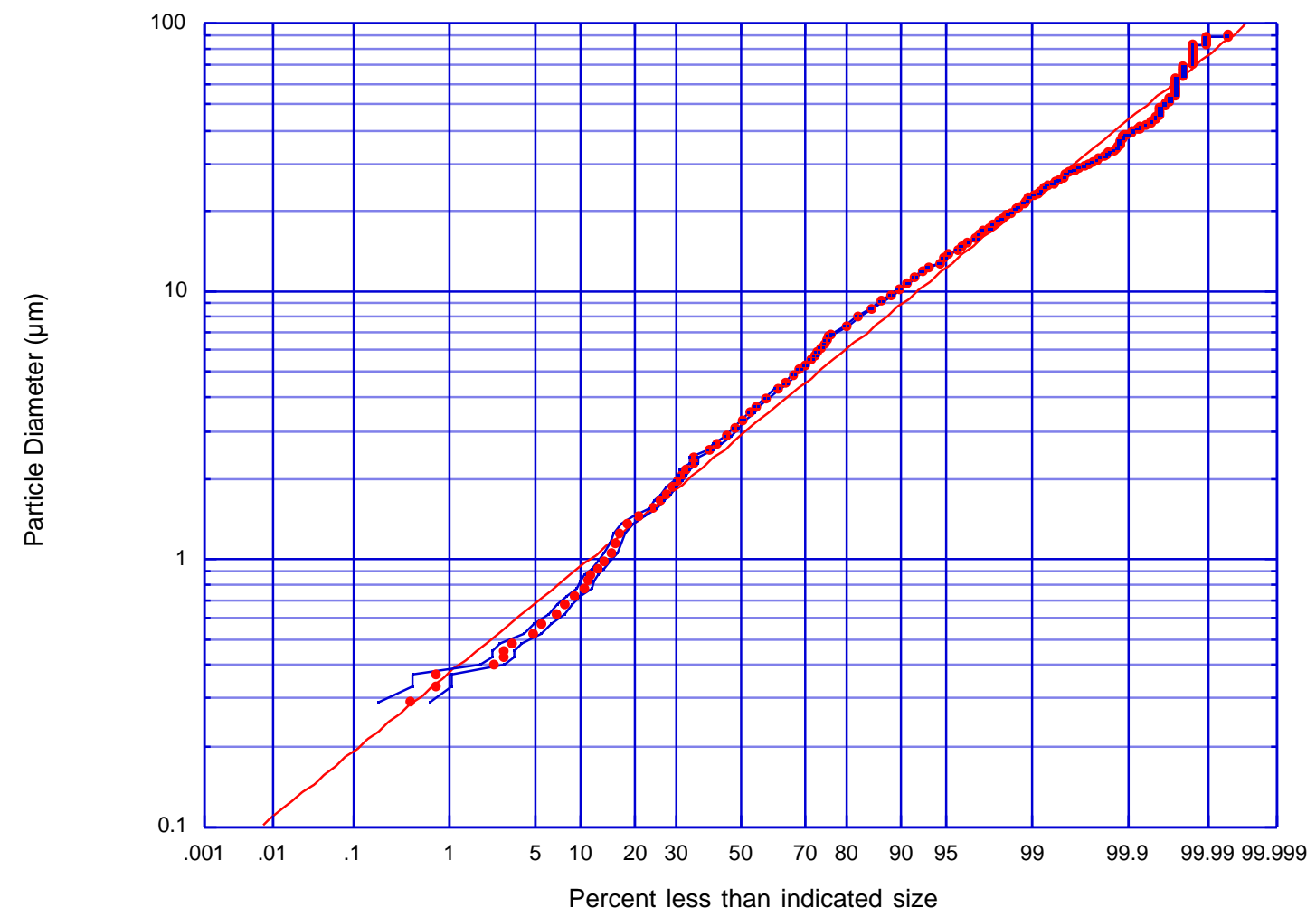

Analysis Summary Table:

\begin{tabular}{|c|c|c|c|c|c|}
\hline \multicolumn{2}{|c|}{$\begin{array}{c}\text { magnification, } \\
\text { number of images }\end{array}$} & \multicolumn{2}{|c|}{ area analyzed $^{1}(\%)$} & counted size range $(\mu \mathrm{m})$ & $\begin{array}{c}\text { total number of } \\
\text { particles (not scaled) }\end{array}$ \\
\hline $50 x$ & 6 & \multicolumn{2}{|r|}{0.894} & $d_{p}>7.01$ & 5328 \\
\hline $100 x$ & 6 & \multicolumn{2}{|r|}{0.224} & $3.88<\mathrm{d}_{\mathrm{p}}<7.01$ & 4319 \\
\hline $200 x$ & 6 & \multicolumn{2}{|r|}{0.0558} & $2.20<d_{p}<3.88$ & 2641 \\
\hline $500 x$ & 6 & \multicolumn{2}{|r|}{0.00893} & $1.35<\mathrm{d}_{\mathrm{p}}<2.20$ & 1001 \\
\hline 1000x, & 6 & \multicolumn{2}{|r|}{0.00217} & $0.00<\mathrm{d}_{\mathrm{p}}<1.35$ & 257 \\
\hline \multicolumn{2}{|c|}{ Totals: } & \multicolumn{2}{|r|}{$1.18 \%$} & - & 13546 \\
\hline \multicolumn{4}{|c|}{ data moments ${ }^{2}:$} & \multicolumn{2}{|c|}{ log-normal fit moments ${ }^{2}$ : } \\
\hline \multicolumn{3}{|c|}{ GMD $(\mu \mathrm{m}):$} & 3.19 & GMD $(\mu \mathrm{m}):$ & 2.89 \\
\hline \multicolumn{2}{|c|}{ GSD: } & & 2.61 & GSD: & 2.41 \\
\hline \multicolumn{3}{|c|}{ lower $95 \%(\mu \mathrm{m})$ : } & 0.47 & $\mathrm{R}^{2}$ & 0.99362 \\
\hline \multirow{2}{*}{\multicolumn{3}{|c|}{ upper $95 \%(\mu \mathrm{m})$ : }} & 21.76 & lower $95 \%(\mu \mathrm{m})$ : & 0.50 \\
\hline & & & & upper $95 \%(\mu \mathrm{m})$ : & 16.82 \\
\hline
\end{tabular}

1. based on overall filter area of $1.59 \times 10^{9} \mu \mathrm{m}^{2}$

2. $\mathrm{GMD}=$ geometric mean diameter, $\mathrm{GSD}=$ geometric standard deviation, $\mathrm{R}=$ linear correlation coefficient

Figure 3.23 Module II, Sample 19 (TSFH10-01) count-based particle size distribution. 


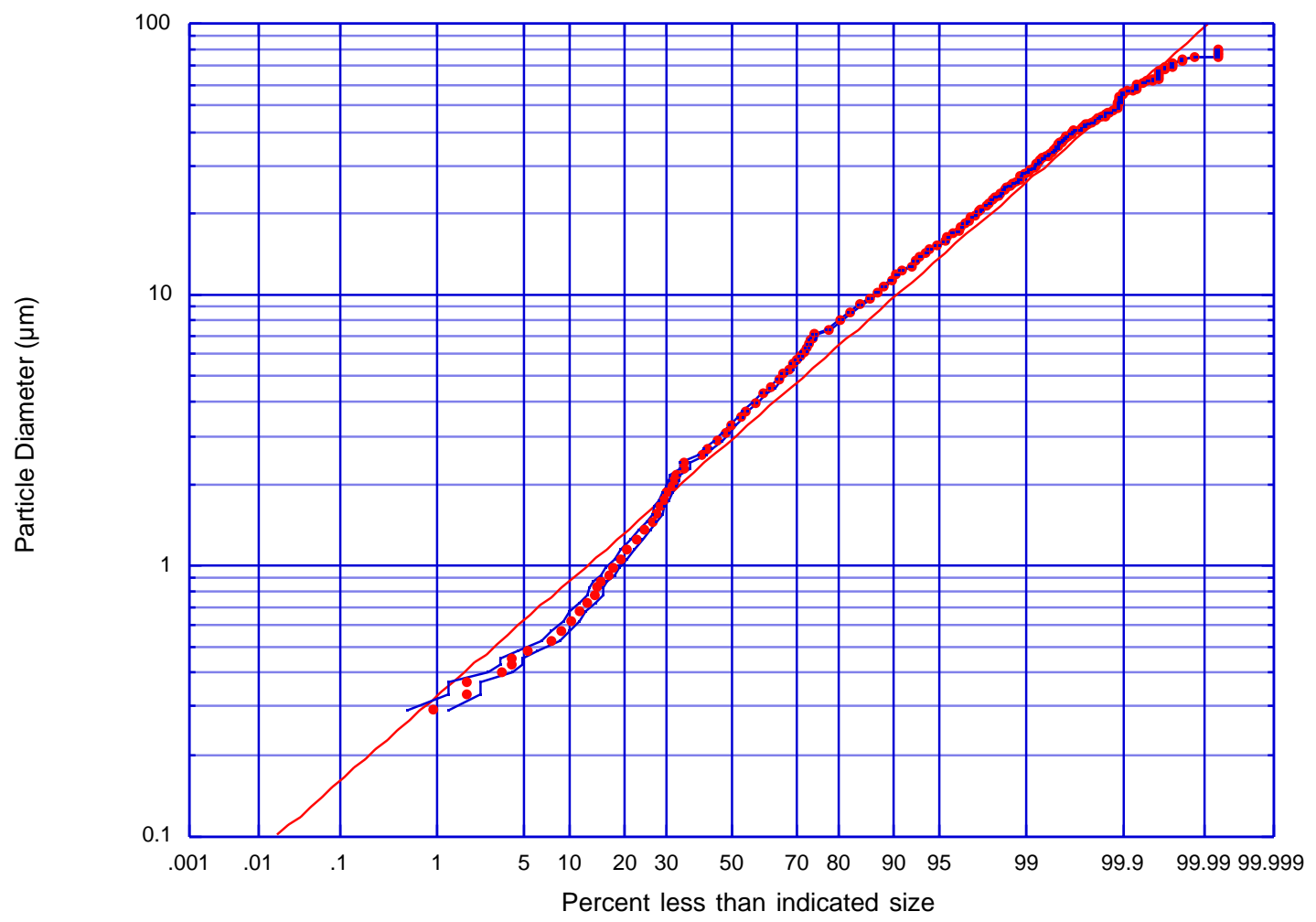

Analysis Summary Table:

\begin{tabular}{|c|c|c|c|c|c|}
\hline \multicolumn{2}{|c|}{$\begin{array}{c}\text { magnification, } \\
\text { number of images }\end{array}$} & \multicolumn{2}{|c|}{ area analyzed $^{1}(\%)$} & counted size range $(\mu \mathrm{m})$ & $\begin{array}{c}\text { total number of } \\
\text { particles (not scaled) }\end{array}$ \\
\hline $50 x$ & 6 & \multicolumn{2}{|r|}{0.894} & $d_{p}>7.31$ & 5418 \\
\hline $100 x$ & 6 & \multicolumn{2}{|r|}{0.224} & $3.93<d_{p}<7.31$ & 3172 \\
\hline $200 x$ & 6 & \multicolumn{2}{|r|}{0.0558} & $2.27<d_{p}<3.93$ & 2189 \\
\hline $500 x$ & 6 & \multicolumn{2}{|r|}{0.00893} & $1.28<\mathrm{d}_{\mathrm{p}}<2.27$ & 582 \\
\hline 1000x, & 6 & \multicolumn{2}{|r|}{0.00217} & $0.00<\mathrm{d}_{\mathrm{p}}<1.28$ & 228 \\
\hline \multicolumn{2}{|c|}{ Totals: } & \multicolumn{2}{|r|}{$1.18 \%$} & - & 11589 \\
\hline \multicolumn{4}{|c|}{ data moments ${ }^{2}:$} & \multicolumn{2}{|c|}{ log-normal fit moments ${ }^{2}$ : } \\
\hline \multicolumn{3}{|c|}{ GMD $(\mu \mathrm{m}):$} & 3.13 & GMD $(\mu \mathrm{m}):$ & 2.93 \\
\hline \multicolumn{2}{|c|}{ GSD: } & & 2.92 & GSD: & 2.56 \\
\hline \multicolumn{3}{|c|}{ lower $95 \%(\mu \mathrm{m})$ : } & 0.37 & $\mathrm{R}^{2}$ & 0.99069 \\
\hline \multirow{2}{*}{\multicolumn{3}{|c|}{ upper $95 \%(\mu \mathrm{m})$ : }} & 26.64 & lower $95 \%(\mu \mathrm{m})$ : & 0.45 \\
\hline & & & & upper $95 \%(\mu \mathrm{m})$ : & 19.22 \\
\hline
\end{tabular}

1. based on overall filter area of $1.59 \times 10^{9} \mu \mathrm{m}^{2}$

2. $\mathrm{GMD}=$ geometric mean diameter, $\mathrm{GSD}=$ geometric standard deviation, $\mathrm{R}=$ linear correlation coefficient

Figure 3.24 Module II, Sample 20 (TSFH13-01) count-based particle size distribution. 


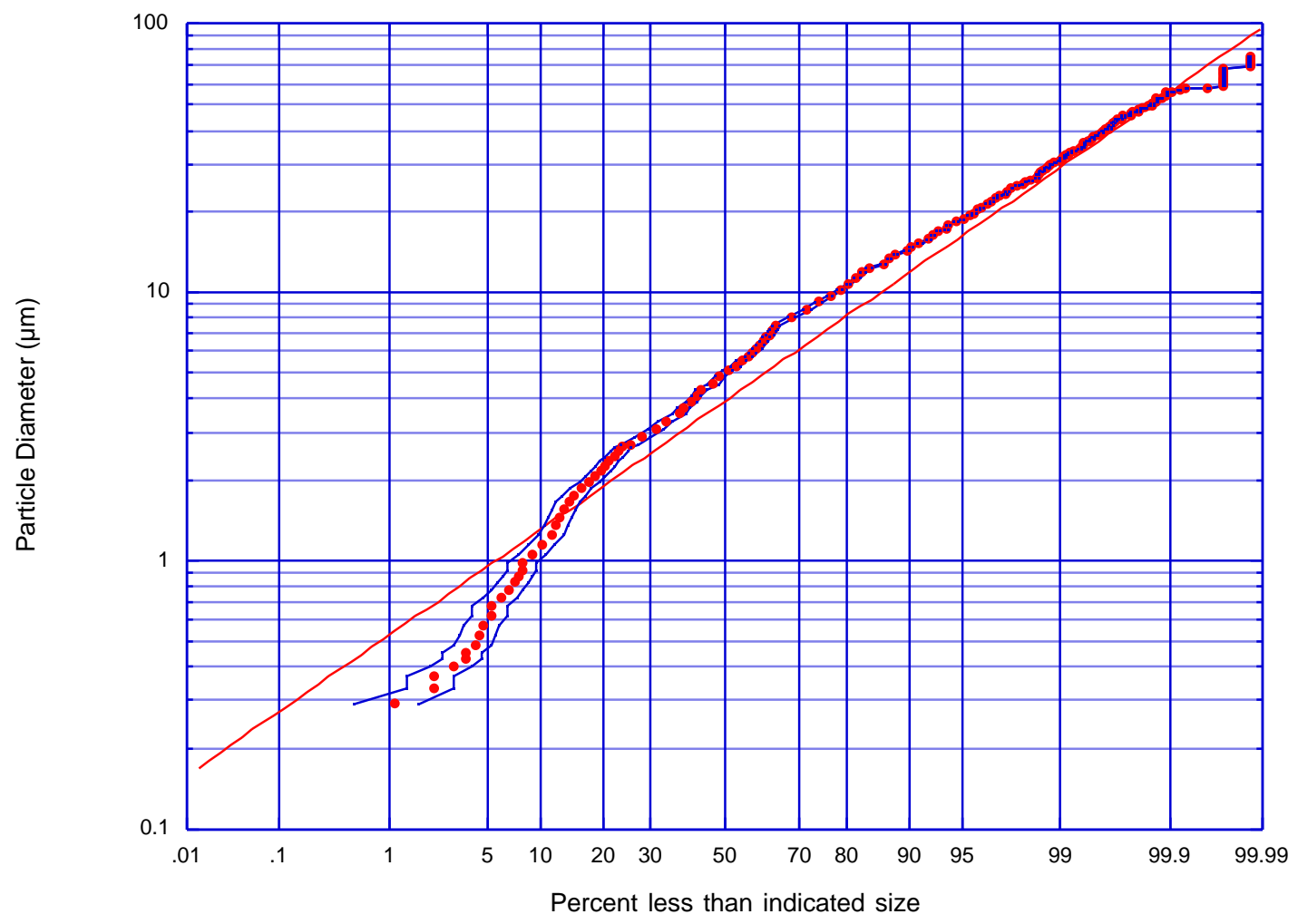

Analysis Summary Table:

\begin{tabular}{|c|c|c|c|c|c|}
\hline \multicolumn{2}{|c|}{$\begin{array}{c}\text { magnification, } \\
\text { number of images }\end{array}$} & \multicolumn{2}{|c|}{ area analyzed $^{1}(\%)$} & counted size range $(\mu \mathrm{m})$ & $\begin{array}{c}\text { total number of } \\
\text { particles (not scaled) }\end{array}$ \\
\hline 50x, & 6 & \multicolumn{2}{|r|}{0.894} & $\mathrm{~d}_{\mathrm{p}}>7.80$ & 3822 \\
\hline $100 x$ & 6 & \multicolumn{2}{|r|}{0.224} & $4.48<\mathrm{d}_{\mathrm{p}}<7.80$ & 1939 \\
\hline $200 x$ & 6 & \multicolumn{2}{|r|}{0.0558} & $2.72<d_{p}<4.48$ & 1043 \\
\hline $500 x$ & 6 & \multicolumn{2}{|r|}{0.00893} & $1.63<\mathrm{d}_{\mathrm{p}}<2.72$ & 301 \\
\hline 1000x, & 6 & \multicolumn{2}{|r|}{0.00217} & $0.00<d_{p}<1.63$ & 82 \\
\hline \multicolumn{2}{|c|}{ Totals: } & \multicolumn{2}{|r|}{$1.18 \%$} & - & 7187 \\
\hline \multicolumn{4}{|c|}{ data moments ${ }^{2}:$} & \multicolumn{2}{|c|}{ log-normal fit moments ${ }^{2}$ : } \\
\hline \multicolumn{3}{|c|}{ GMD $(\mu \mathrm{m}):$} & 4.65 & GMD $(\mu \mathrm{m}):$ & 3.92 \\
\hline \multicolumn{2}{|c|}{ GSD: } & & 2.72 & GSD: & 2.37 \\
\hline \multicolumn{3}{|c|}{ lower $95 \%(\mu \mathrm{m})$ : } & 0.63 & $\mathrm{R}^{2}:$ & 0.97932 \\
\hline \multicolumn{3}{|c|}{ upper $95 \%(\mu \mathrm{m})$ : } & 34.49 & lower $95 \%(\mu \mathrm{m})$ : & 0.70 \\
\hline & & & & upper $95 \%(\mu \mathrm{m})$ : & 22.00 \\
\hline
\end{tabular}

1. based on overall filter area of $1.59 \times 10^{9} \mu \mathrm{m}^{2}$

2. $\mathrm{GMD}=$ geometric mean diameter, $\mathrm{GSD}=$ geometric standard deviation, $\mathrm{R}=$ linear correlation coefficient

Figure 3.25 Module II, Sample 20A (TSFH15-01) count-based particle size distribution. 


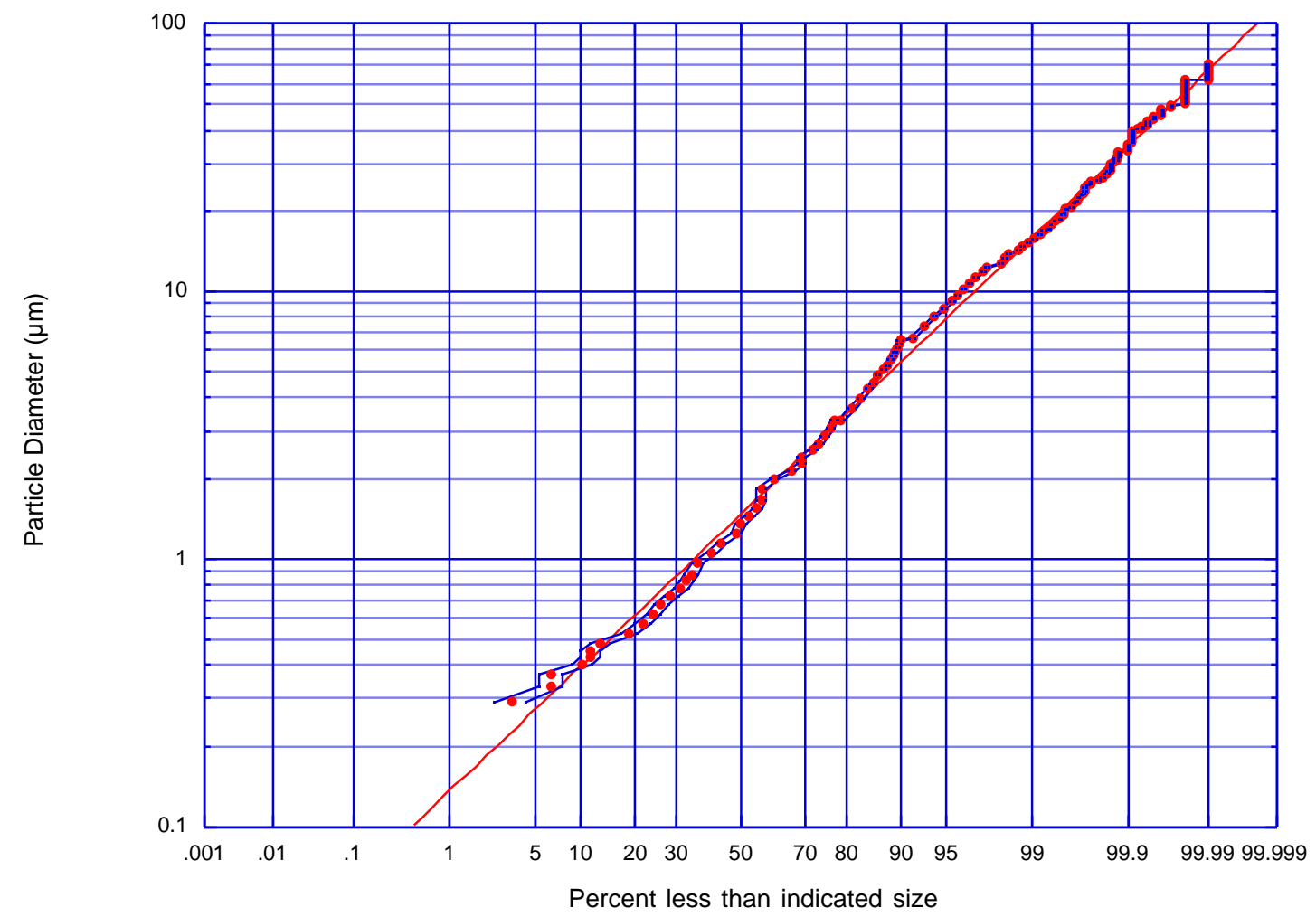

Analysis Summary Table:

\begin{tabular}{|c|c|c|c|c|c|}
\hline \multicolumn{2}{|c|}{$\begin{array}{c}\text { magnification, } \\
\text { number of images }\end{array}$} & \multicolumn{2}{|c|}{ area analyzed $^{1}(\%)$} & counted size range $(\mu \mathrm{m})$ & $\begin{array}{c}\text { total number of } \\
\text { particles (not scaled) }\end{array}$ \\
\hline $50 x$ & 6 & \multicolumn{2}{|r|}{0.894} & $d_{p}>6.72$ & 1330 \\
\hline $100 x$ & 6 & \multicolumn{2}{|r|}{0.224} & $3.53<d_{p}<6.72$ & 1035 \\
\hline $200 x$ & 6 & \multicolumn{2}{|r|}{0.0558} & $1.77<\mathrm{d}_{\mathrm{p}}<3.53$ & 912 \\
\hline $500 x$ & 6 & \multicolumn{2}{|r|}{0.00893} & $0.91<\mathrm{d}_{\mathrm{p}}<1.77$ & 532 \\
\hline 1000x, & 6 & \multicolumn{2}{|r|}{0.00217} & $0.00<\mathrm{d}_{\mathrm{p}}<0.91$ & 239 \\
\hline \multicolumn{2}{|c|}{ Totals: } & \multicolumn{2}{|r|}{$1.18 \%$} & - & 4048 \\
\hline \multicolumn{4}{|c|}{ data moments ${ }^{2}:$} & \multicolumn{2}{|c|}{ log-normal fit moments ${ }^{2}$ : } \\
\hline \multicolumn{3}{|c|}{ GMD $(\mu \mathrm{m}):$} & 1.52 & GMD $(\mu \mathrm{m}):$ & 1.47 \\
\hline \multicolumn{2}{|c|}{ GSD: } & & 2.73 & GSD: & 2.79 \\
\hline \multicolumn{3}{|c|}{ lower $95 \%(\mu \mathrm{m})$ : } & 0.20 & $\mathrm{R}^{2}$ & 0.99787 \\
\hline \multirow{2}{*}{\multicolumn{3}{|c|}{ upper $95 \%(\mu \mathrm{m})$ : }} & 11.38 & lower $95 \%(\mu \mathrm{m})$ : & 0.19 \\
\hline & & & & upper $95 \%(\mu \mathrm{m})$ : & 11.46 \\
\hline
\end{tabular}

1. based on overall filter area of $1.59 \times 10^{9} \mu \mathrm{m}^{2}$

2. $\mathrm{GMD}=$ geometric mean diameter, $\mathrm{GSD}=$ geometric standard deviation, $\mathrm{R}=$ linear correlation coefficient

Figure 3.26 Module II, Sample 20B (TSFH16-01) count-based particle size distribution. 


\subsubsection{Module III Results}

Figure 3.27 through Figure 3.38 display details of particle size distributions for corresponding collection locations of Module III. Table 3.3 gives a summary of Module III results.

Table 3.3 Summary of dust collected from locations in Tore Supra Module III.

\begin{tabular}{|c|c|c|c|c|c|c|}
\hline \multirow{2}{*}{$\begin{array}{c}\text { Poloidal } \\
\text { Location }\end{array}$} & Sample & Filter ID & $\begin{array}{c}\text { Sampled } \\
\text { Area }\left(\mathrm{cm}^{2}\right)\end{array}$ & $\begin{array}{c}\text { Collected } \\
\text { Mass }(\mathrm{mg})\end{array}$ & \multicolumn{2}{|c|}{$\begin{array}{c}\text { Count-based Size } \\
\text { Distribution Parameters }\end{array}$} \\
\hline \hline 1 & & & GMD $(\mu \mathrm{m})$ & GSD \\
\hline 2 & 21 & TSFH05-02 & 621.2 & 0.5 & 4.40 & 2.87 \\
\hline 4 & 23 & TSFH23-02 & 1,553 & 1.6 & 2.81 & 3.11 \\
\hline 3 & 24 & TSFH06-02 & 689.0 & 2.0 & 3.38 & 2.85 \\
\hline 6 & 25 & TSFH11-02 & 1,800 & 0.7 & 2.74 & 2.92 \\
\hline 5 & 26 & TSFH08-02 & 658.0 & 0.2 & 4.00 & 3.15 \\
\hline 7 & 27 & TSFH09-02 & 428.0 & 1.4 & 4.63 & 2.91 \\
\hline 8 & 28 & TSFH07-02 & 84.00 & 0.3 & 4.19 & 3.04 \\
\hline 10 & 29 & TSFH22-02 & 1,429 & 1.4 & 3.65 & 3.15 \\
\hline 9 & 30 & TSFH25-02 & 294.0 & 0.3 & 3.28 & 3.23 \\
\hline 11 & $30 \mathrm{~A}$ & TSFH24-02 & 160.0 & $34.7^{*}$ & 1.96 & 2.63 \\
\hline 12 & $30 \mathrm{~B}$ & TSFH21-02 & 480.0 & 38.3 & 1.66 & 2.94 \\
\hline
\end{tabular}

*- filter partially broken 


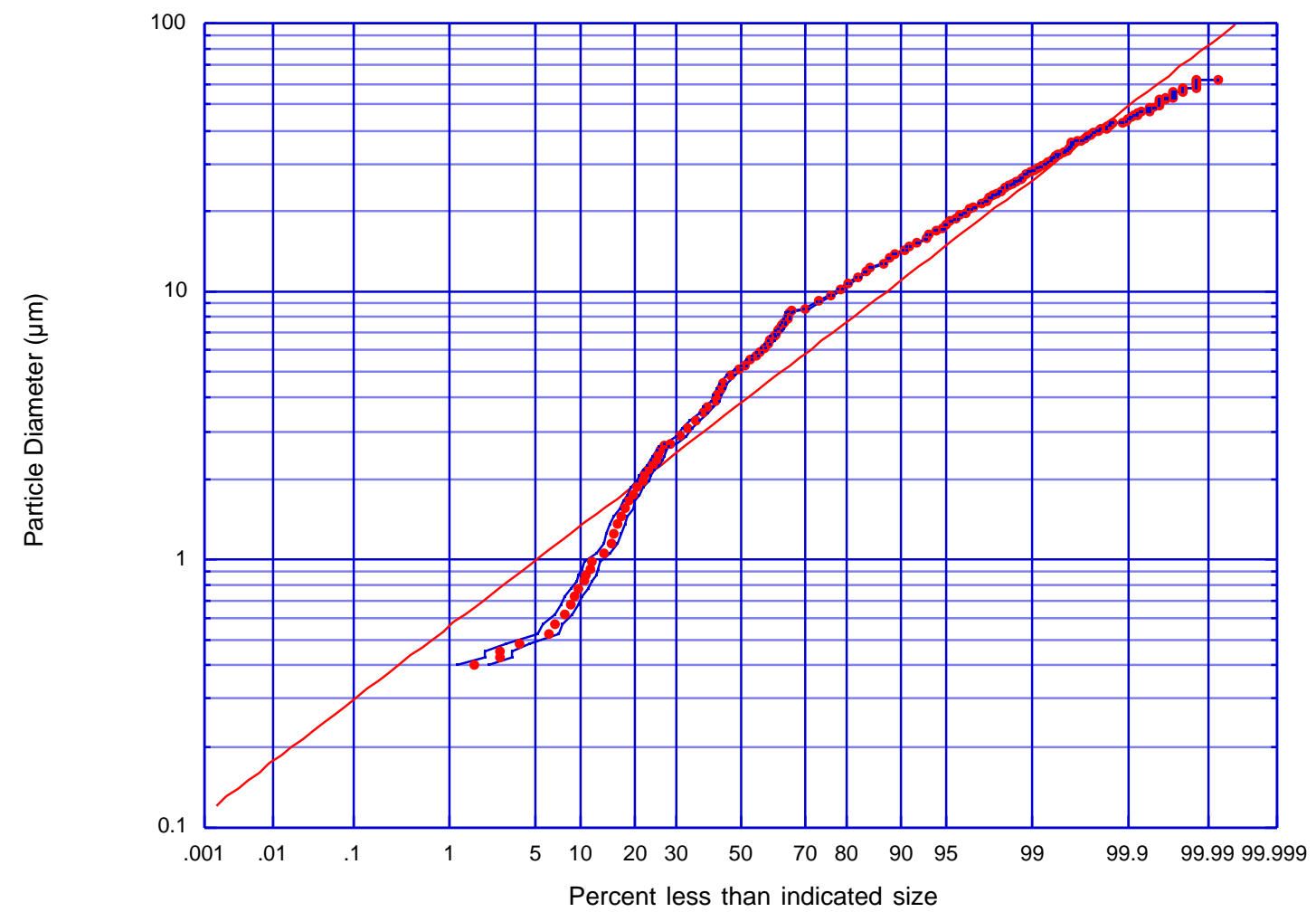

Analysis Summary Table:

\begin{tabular}{|c|c|c|c|c|c|}
\hline \multicolumn{2}{|c|}{$\begin{array}{c}\text { magnification, } \\
\text { number of images }\end{array}$} & \multicolumn{2}{|c|}{ area analyzed $^{1}(\%)$} & counted size range $(\mu \mathrm{m})$ & $\begin{array}{c}\text { total number of } \\
\text { particles (not scaled) }\end{array}$ \\
\hline $50 x$ & 6 & \multicolumn{2}{|r|}{0.894} & $d_{p}>8.64$ & 6123 \\
\hline $100 x$ & 6 & \multicolumn{2}{|r|}{0.224} & $4.75<d_{p}<8.64$ & 3111 \\
\hline $200 x$ & 6 & \multicolumn{2}{|r|}{0.0558} & $2.77<d_{p}<4.75$ & 1432 \\
\hline $500 x$ & 6 & \multicolumn{2}{|r|}{0.00893} & $1.62<d_{p}<2.77$ & 456 \\
\hline 1000x, & 6 & \multicolumn{2}{|r|}{0.00217} & $0.00<\mathrm{d}_{\mathrm{p}}<1.62$ & 192 \\
\hline \multicolumn{2}{|c|}{ Totals: } & \multicolumn{2}{|r|}{$1.18 \%$} & - & 11314 \\
\hline \multicolumn{4}{|c|}{ data moments ${ }^{2}:$} & \multicolumn{2}{|c|}{ log-normal fit moments ${ }^{2}$ : } \\
\hline \multicolumn{3}{|c|}{ GMD $(\mu \mathrm{m}):$} & 4.40 & GMD $(\mu \mathrm{m}):$ & 3.83 \\
\hline \multicolumn{2}{|c|}{ GSD: } & & 2.87 & GSD: & 2.29 \\
\hline \multicolumn{3}{|c|}{ lower $95 \%(\mu \mathrm{m})$ : } & 0.53 & $\mathrm{R}^{2}$ & 0.96637 \\
\hline \multirow{2}{*}{\multicolumn{3}{|c|}{ upper $95 \%(\mu \mathrm{m})$ : }} & 36.15 & lower $95 \%(\mu \mathrm{m})$ : & 0.73 \\
\hline & & & & upper $95 \%(\mu \mathrm{m})$ : & 20.02 \\
\hline
\end{tabular}

1. based on overall filter area of $1.59 \times 10^{9} \mu \mathrm{m}^{2}$

2. $\mathrm{GMD}=$ geometric mean diameter, $\mathrm{GSD}=$ geometric standard deviation, $\mathrm{R}=$ linear correlation coefficient

Figure 3.27 Module III, Sample 21 (TSFH05-02) count-based particle size distribution. 


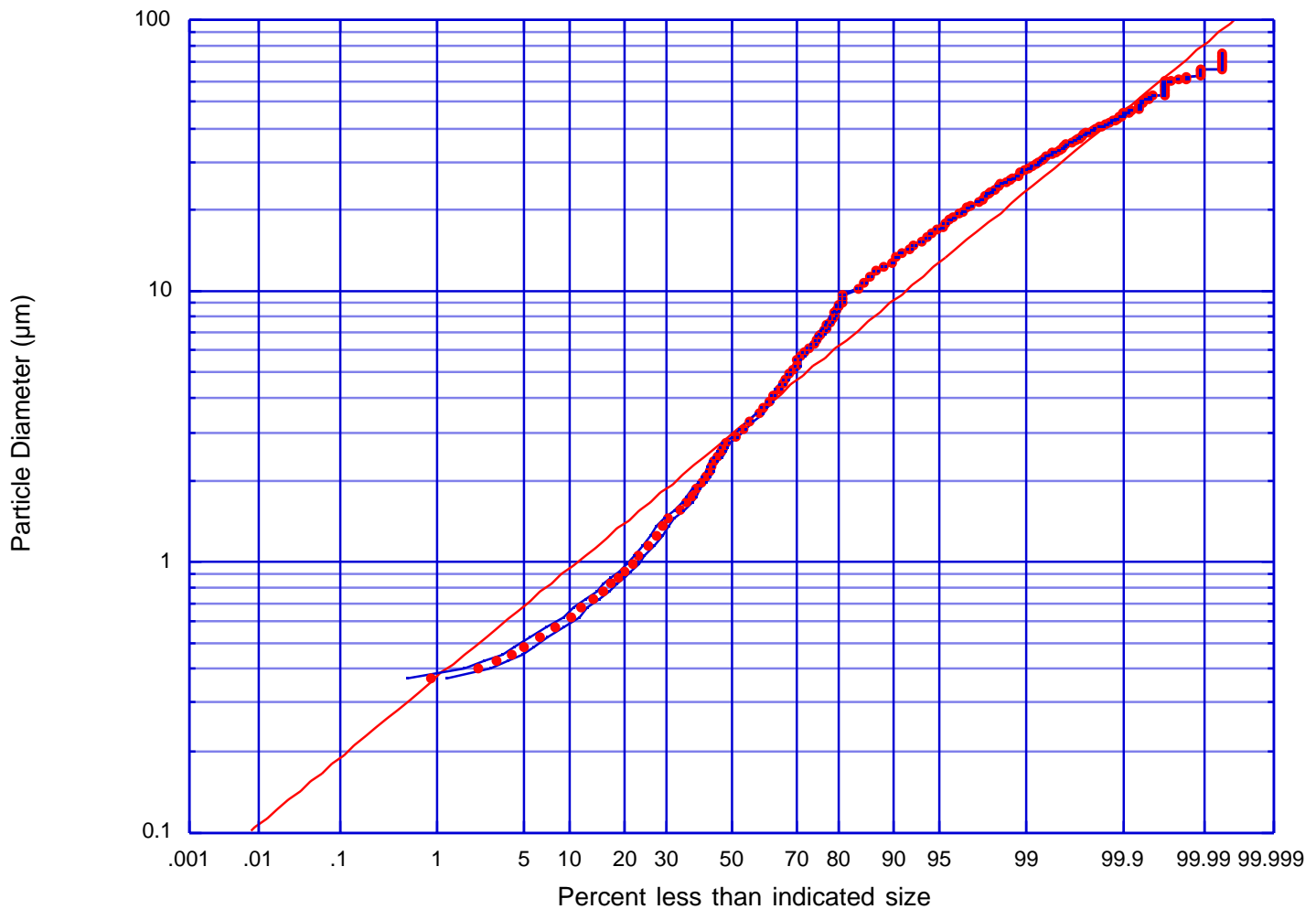

Analysis Summary Table:

\begin{tabular}{|c|c|c|c|c|c|}
\hline \multicolumn{2}{|c|}{$\begin{array}{c}\text { magnification, } \\
\text { number of images }\end{array}$} & \multicolumn{2}{|c|}{$\operatorname{area}_{\text {analyzed }}{ }^{1}(\%)$} & counted size range $(\mu \mathrm{m})$ & $\begin{array}{c}\text { total number of } \\
\text { particles (not scaled) }\end{array}$ \\
\hline $50 x$ & 6 & & 0.894 & $d_{p}>9.52$ & 5227 \\
\hline $100 x$ & 6 & & 0.224 & $5.34<d_{p}<9.52$ & 2929 \\
\hline $200 x$ & 6 & & 0.0558 & $2.80<\mathrm{d}_{\mathrm{p}}<5.34$ & 2475 \\
\hline $500 x$ & 6 & & 0.00893 & $1.52<\mathrm{d}_{\mathrm{p}}<2.80$ & 966 \\
\hline $1000 x$, & 6 & & 0.00217 & $0.00<\mathrm{d}_{\mathrm{p}}<1.52$ & 432 \\
\hline \multicolumn{2}{|c|}{ Totals: } & & $1.18 \%$ & - & 12029 \\
\hline \multicolumn{4}{|c|}{ data moments ${ }^{2}$ : } & \multicolumn{2}{|c|}{ log-normal fit moments ${ }^{2}$ : } \\
\hline \multicolumn{3}{|c|}{ GMD $(\mu \mathrm{m}):$} & 2.81 & GMD $(\mu \mathrm{m}):$ & 2.89 \\
\hline \multicolumn{3}{|c|}{ GSD: } & 3.11 & GSD: & 2.52 \\
\hline \multicolumn{3}{|c|}{ lower $95 \%(\mu \mathrm{m})$ : } & 0.29 & $\mathrm{R}^{2}:$ & 0.9746 \\
\hline \multicolumn{3}{|c|}{ upper $95 \%(\mu \mathrm{m})$ : } & 27.20 & lower $95 \%(\mu \mathrm{m})$ : & 0.46 \\
\hline & & & & upper $95 \%(\mu \mathrm{m})$ : & 18.35 \\
\hline
\end{tabular}

1. based on overall filter area of $1.59 \times 10^{9} \mu \mathrm{m}^{2}$

2. $\mathrm{GMD}=$ geometric mean diameter, GSD = geometric standard deviation, $\mathrm{R}=$ linear correlation coefficient

Figure 3.28 Module III, Sample 22 (TSFH23-02) count-based particle size distribution. 


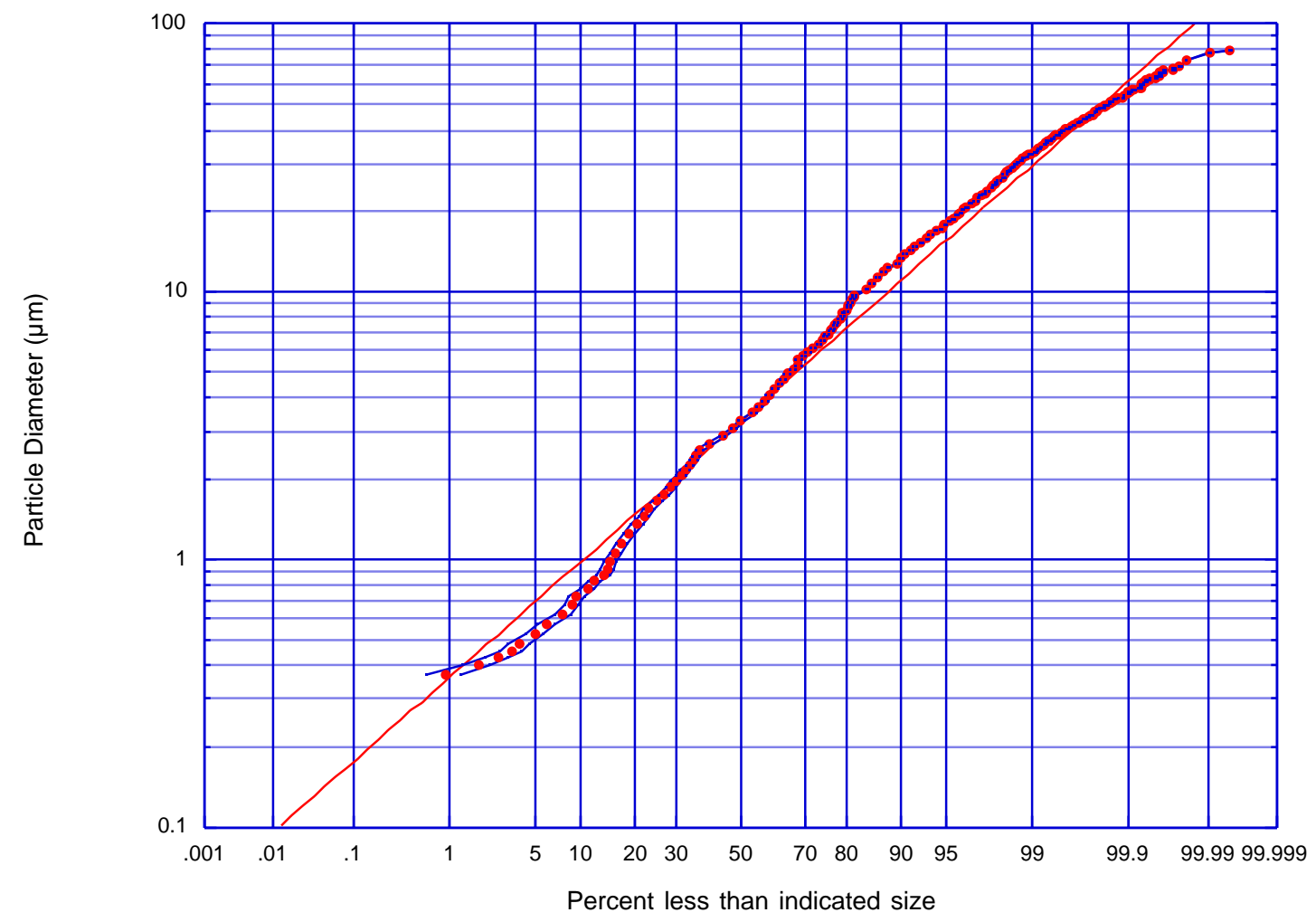

Analysis Summary Table:

\begin{tabular}{|c|c|c|c|c|c|}
\hline \multicolumn{2}{|c|}{$\begin{array}{c}\text { magnification, } \\
\text { number of images }\end{array}$} & \multicolumn{2}{|c|}{ area analyzed $^{1}(\%)$} & counted size range $(\mu \mathrm{m})$ & $\begin{array}{c}\text { total number of } \\
\text { particles (not scaled) }\end{array}$ \\
\hline $50 x$ & 6 & \multicolumn{2}{|r|}{0.894} & $d_{p}>9.75$ & 5785 \\
\hline $100 x$ & 6 & \multicolumn{2}{|r|}{0.224} & $5.16<d_{p}<9.75$ & 4162 \\
\hline $200 x$ & 6 & \multicolumn{2}{|r|}{0.0558} & $2.67<d_{p}<5.16$ & 3257 \\
\hline $500 x$ & 6 & \multicolumn{2}{|r|}{0.00893} & $1.62<d_{p}<2.67$ & 1237 \\
\hline 1000x, & 6 & \multicolumn{2}{|r|}{0.00217} & $0.00<\mathrm{d}_{\mathrm{p}}<1.62$ & 425 \\
\hline \multicolumn{2}{|c|}{ Totals: } & \multicolumn{2}{|r|}{$1.18 \%$} & - & 14866 \\
\hline \multicolumn{4}{|c|}{ data moments ${ }^{2}:$} & \multicolumn{2}{|c|}{ log-normal fit moments ${ }^{2}$ : } \\
\hline \multicolumn{3}{|c|}{ GMD $(\mu \mathrm{m}):$} & 3.38 & GMD $(\mu \mathrm{m}):$ & 3.28 \\
\hline \multicolumn{2}{|c|}{ GSD: } & & 2.85 & GSD: & 2.58 \\
\hline \multicolumn{3}{|c|}{ lower $95 \%(\mu \mathrm{m})$ : } & 0.42 & $\mathrm{R}^{2}$ & 0.98988 \\
\hline \multirow{2}{*}{\multicolumn{3}{|c|}{ upper $95 \%(\mu \mathrm{m})$ : }} & 27.51 & lower $95 \%(\mu \mathrm{m})$ : & 0.49 \\
\hline & & & & upper $95 \%(\mu \mathrm{m})$ : & 21.80 \\
\hline
\end{tabular}

1. based on overall filter area of $1.59 \times 10^{9} \mu \mathrm{m}^{2}$

2. $\mathrm{GMD}=$ geometric mean diameter, $\mathrm{GSD}=$ geometric standard deviation, $\mathrm{R}=$ linear correlation coefficient

Figure 3.29 Module III, Sample 23 (TSFH06-02) count-based particle size distribution. 


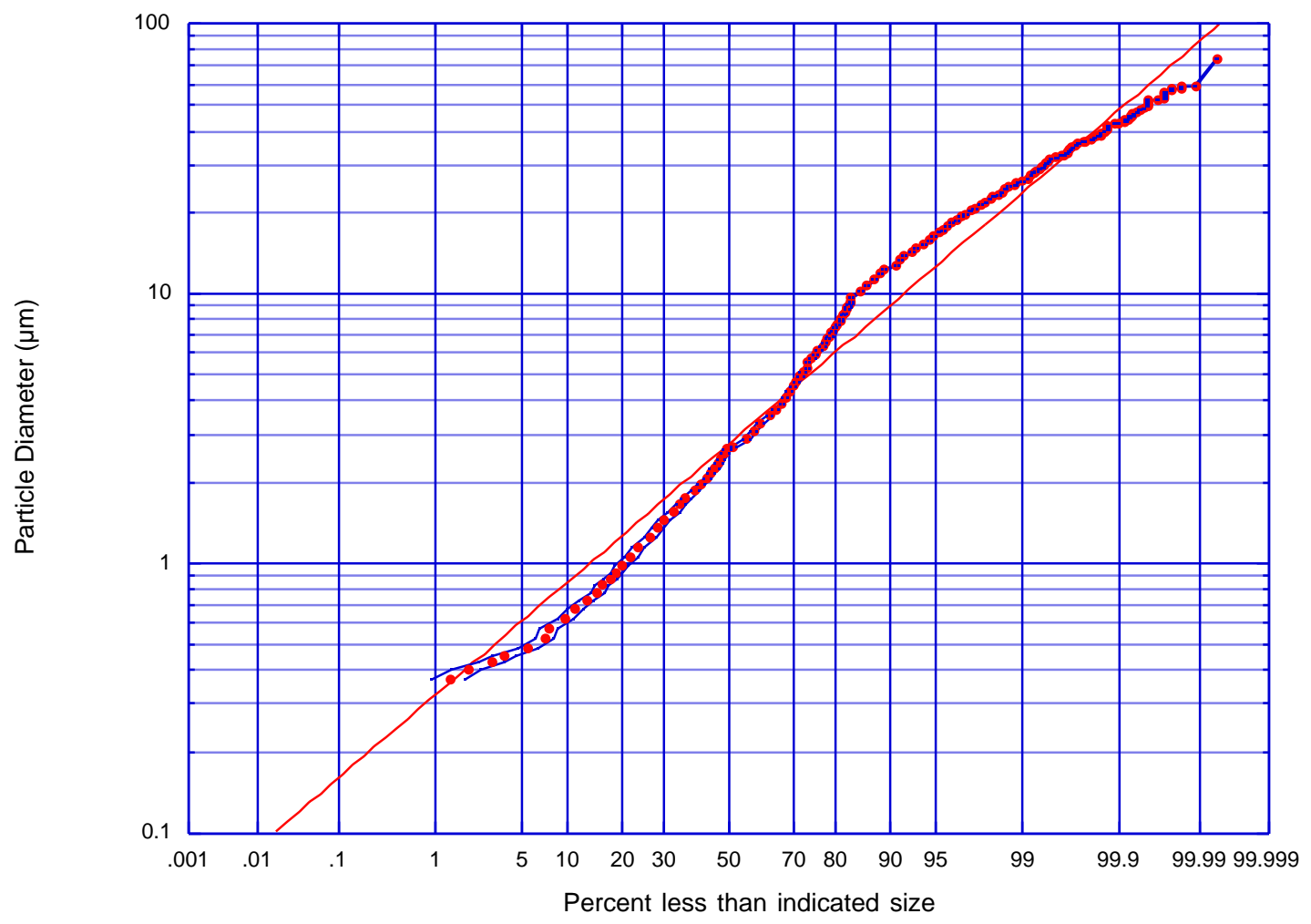

Analysis Summary Table:

\begin{tabular}{|c|c|c|c|c|c|}
\hline \multicolumn{2}{|c|}{$\begin{array}{c}\text { magnification, } \\
\text { number of images }\end{array}$} & \multicolumn{2}{|c|}{ area analyzed $^{1}(\%)$} & counted size range $(\mu \mathrm{m})$ & $\begin{array}{c}\text { total number of } \\
\text { particles (not scaled) }\end{array}$ \\
\hline $50 x$ & 6 & \multicolumn{2}{|r|}{0.894} & $d_{p}>9.49$ & 4676 \\
\hline $100 x$ & 6 & \multicolumn{2}{|r|}{0.224} & $5.24<d_{p}<9.49$ & 2474 \\
\hline $200 x$ & 6 & \multicolumn{2}{|r|}{0.0558} & $2.73<d_{p}<5.24$ & 2324 \\
\hline $500 x$ & 6 & \multicolumn{2}{|r|}{0.00893} & $1.54<\mathrm{d}_{\mathrm{p}}<2.73$ & 978 \\
\hline 1000x, & 6 & \multicolumn{2}{|r|}{0.00217} & $0.00<\mathrm{d}_{\mathrm{p}}<1.54$ & 407 \\
\hline \multicolumn{2}{|c|}{ Totals: } & \multicolumn{2}{|r|}{$1.18 \%$} & - & 10859 \\
\hline \multicolumn{4}{|c|}{ data moments ${ }^{2}:$} & \multicolumn{2}{|c|}{ log-normal fit moments ${ }^{2}$ : } \\
\hline \multicolumn{3}{|c|}{ GMD $(\mu \mathrm{m}):$} & 2.74 & GMD $(\mu \mathrm{m}):$ & 2.78 \\
\hline \multicolumn{2}{|c|}{ GSD: } & & 2.92 & GSD: & 2.52 \\
\hline \multicolumn{3}{|c|}{ lower $95 \%(\mu \mathrm{m})$ : } & 0.32 & $\mathrm{R}^{2}$ & 0.98184 \\
\hline \multirow{2}{*}{\multicolumn{3}{|c|}{ upper $95 \%(\mu \mathrm{m})$ : }} & 23.46 & lower $95 \%(\mu \mathrm{m})$ : & 0.44 \\
\hline & & & & upper $95 \%(\mu \mathrm{m})$ : & 17.61 \\
\hline
\end{tabular}

1. based on overall filter area of $1.59 \times 10^{9} \mu \mathrm{m}^{2}$

2. $\mathrm{GMD}=$ geometric mean diameter, $\mathrm{GSD}=$ geometric standard deviation, $\mathrm{R}=$ linear correlation coefficient

Figure 3.30 Module III, Sample 24 (TSFH11-02) count-based particle size distribution. 


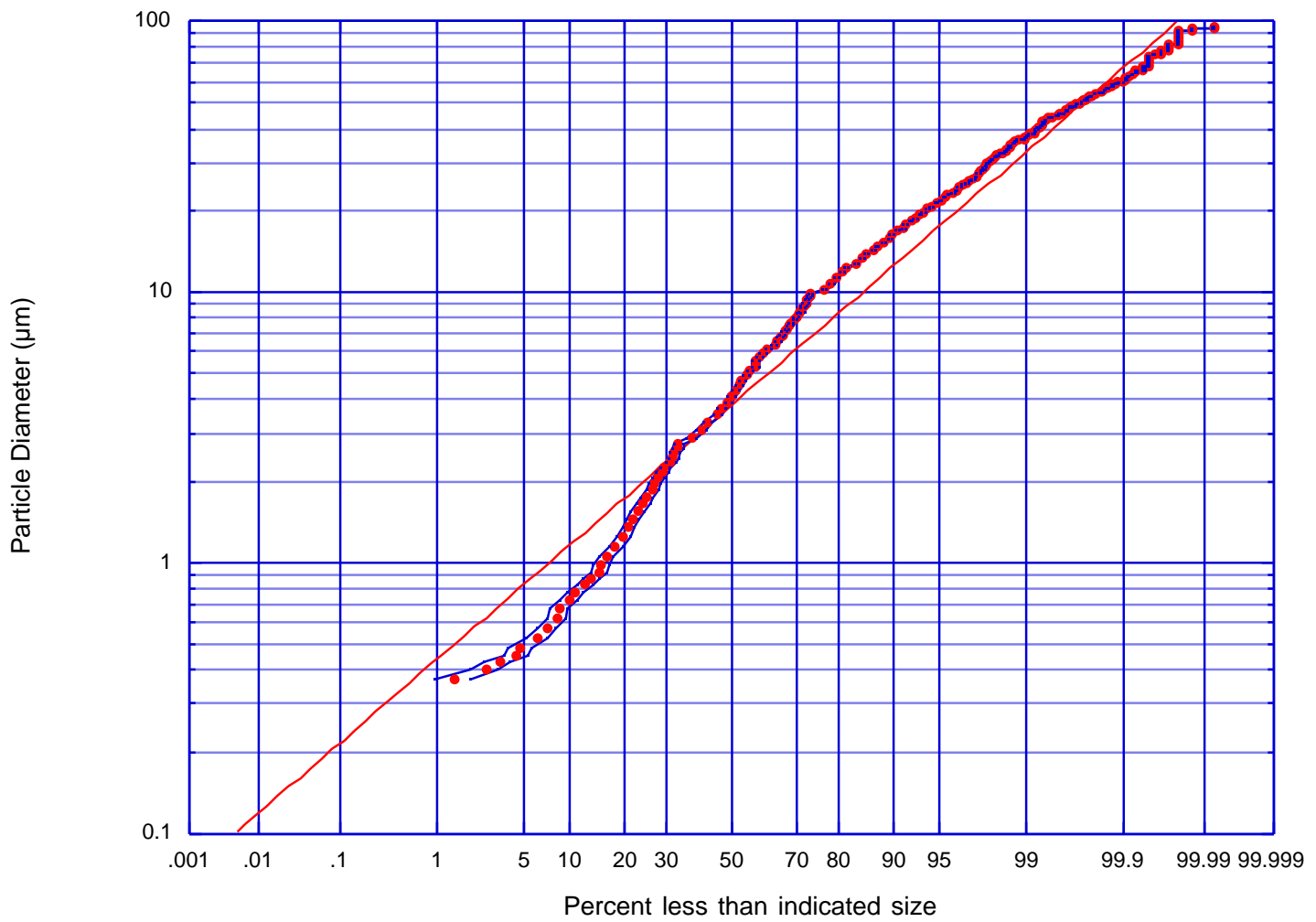

Analysis Summary Table:

\begin{tabular}{|c|c|c|c|c|c|}
\hline \multicolumn{2}{|c|}{$\begin{array}{c}\text { magnification, } \\
\text { number of images }\end{array}$} & \multicolumn{2}{|c|}{ area analyzed $^{1}(\%)$} & counted size range $(\mu \mathrm{m})$ & $\begin{array}{c}\text { total number of } \\
\text { particles (not scaled) }\end{array}$ \\
\hline $50 x$ & 6 & \multicolumn{2}{|r|}{0.894} & $d_{p}>10.09$ & 5252 \\
\hline $100 x$ & 6 & \multicolumn{2}{|r|}{0.224} & $5.35<\mathrm{d}_{\mathrm{p}}<10.09$ & 3025 \\
\hline $200 x$ & 6 & \multicolumn{2}{|r|}{0.0558} & $2.87<\mathrm{d}_{\mathrm{p}}<5.35$ & 1926 \\
\hline $500 x$ & 6 & \multicolumn{2}{|r|}{0.00893} & $1.67<\mathrm{d}_{\mathrm{p}}<2.87$ & 521 \\
\hline 1000x, & 6 & \multicolumn{2}{|r|}{0.00217} & $0.00<\mathrm{d}_{\mathrm{p}}<1.67$ & 241 \\
\hline \multicolumn{2}{|c|}{ Totals: } & \multicolumn{2}{|r|}{$1.18 \%$} & - & 10965 \\
\hline \multicolumn{4}{|c|}{ data moments ${ }^{2}:$} & \multicolumn{2}{|c|}{ log-normal fit moments ${ }^{2}$ : } \\
\hline \multicolumn{3}{|c|}{ GMD $(\mu \mathrm{m}):$} & 4.00 & GMD $(\mu \mathrm{m}):$ & 3.81 \\
\hline \multicolumn{2}{|c|}{ GSD: } & & 3.15 & GSD: & 2.53 \\
\hline \multicolumn{3}{|c|}{ lower $95 \%(\mu \mathrm{m})$ : } & 0.40 & $\mathrm{R}^{2}$ & 0.97966 \\
\hline \multirow{2}{*}{\multicolumn{3}{|c|}{ upper $95 \%(\mu \mathrm{m})$ : }} & 39.80 & lower $95 \%(\mu \mathrm{m})$ : & 0.59 \\
\hline & & & & upper $95 \%(\mu \mathrm{m})$ : & 24.48 \\
\hline
\end{tabular}

1. based on overall filter area of $1.59 \times 10^{9} \mu \mathrm{m}^{2}$

2. $\mathrm{GMD}=$ geometric mean diameter, $\mathrm{GSD}=$ geometric standard deviation, $\mathrm{R}=$ linear correlation coefficient

Figure 3.31 Module III, Sample 25 (TSFH08-02) count-based particle size distribution. 


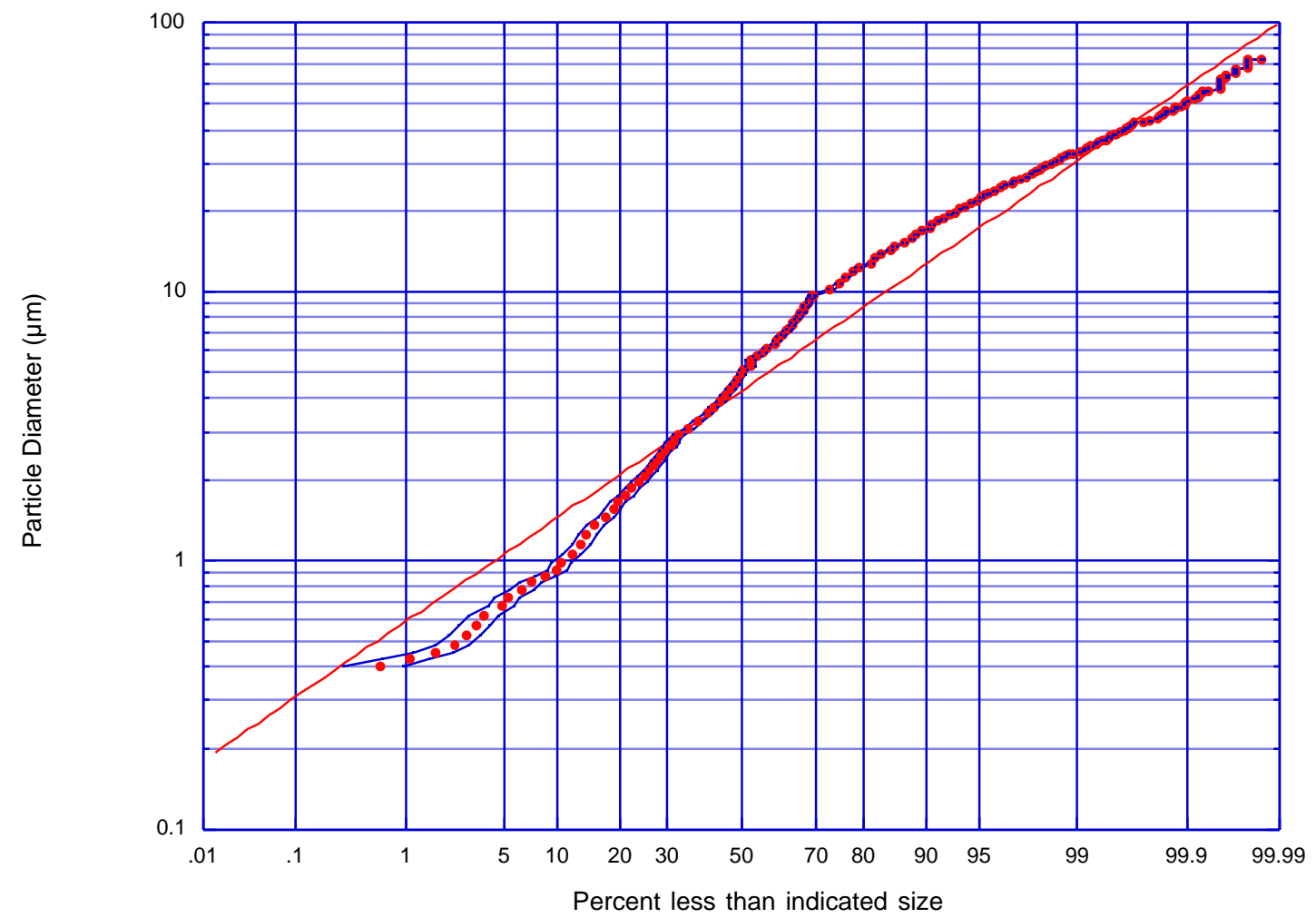

Analysis Summary Table:

\begin{tabular}{|c|c|c|c|c|c|}
\hline \multicolumn{2}{|c|}{$\begin{array}{c}\text { magnification, } \\
\text { number of images }\end{array}$} & \multicolumn{2}{|c|}{ area analyzed $^{1}(\%)$} & counted size range $(\mu \mathrm{m})$ & $\begin{array}{c}\text { total number of } \\
\text { particles (not scaled) }\end{array}$ \\
\hline $50 x$ & 6 & \multicolumn{2}{|r|}{0.894} & $d_{p}>9.87$ & 5565 \\
\hline $100 x$ & 6 & \multicolumn{2}{|r|}{0.224} & $5.26<d_{p}<9.87$ & 2940 \\
\hline $200 x$ & 6 & \multicolumn{2}{|r|}{0.0558} & $3.00<d_{p}<5.26$ & 1630 \\
\hline $500 x$ & 6 & \multicolumn{2}{|r|}{0.00893} & $1.88<\mathrm{d}_{\mathrm{p}}<3.00$ & 585 \\
\hline $1000 x$, & 6 & \multicolumn{2}{|r|}{0.00217} & $0.00<\mathrm{d}_{\mathrm{p}}<1.88$ & 222 \\
\hline \multicolumn{2}{|c|}{ Totals: } & \multicolumn{2}{|r|}{$1.18 \%$} & - & 10942 \\
\hline \multicolumn{4}{|c|}{ data moments ${ }^{2}:$} & \multicolumn{2}{|c|}{ log-normal fit moments ${ }^{2}$ : } \\
\hline \multicolumn{3}{|c|}{ GMD $(\mu \mathrm{m}):$} & 4.63 & GMD $(\mu \mathrm{m}):$ & 4.26 \\
\hline \multicolumn{2}{|c|}{ GSD: } & & 2.91 & GSD: & 2.34 \\
\hline \multicolumn{3}{|c|}{ lower $95 \%(\mu \mathrm{m})$ : } & 0.54 & $\mathrm{R}^{2}$ & 0.9745 \\
\hline \multicolumn{3}{|c|}{ upper $95 \%(\mu \mathrm{m})$ : } & 39.28 & lower $95 \%(\mu \mathrm{m})$ : & 0.78 \\
\hline & & & & upper $95 \%(\mu \mathrm{m})$ : & 23.27 \\
\hline
\end{tabular}

1. based on overall filter area of $1.59 \times 10^{9} \mu \mathrm{m}^{2}$

2. $\mathrm{GMD}=$ geometric mean diameter, $\mathrm{GSD}=$ geometric standard deviation, $\mathrm{R}=$ linear correlation coefficient

Figure 3.32 Module III, Sample 26 (TSFH10-02) count-based particle size distribution. 


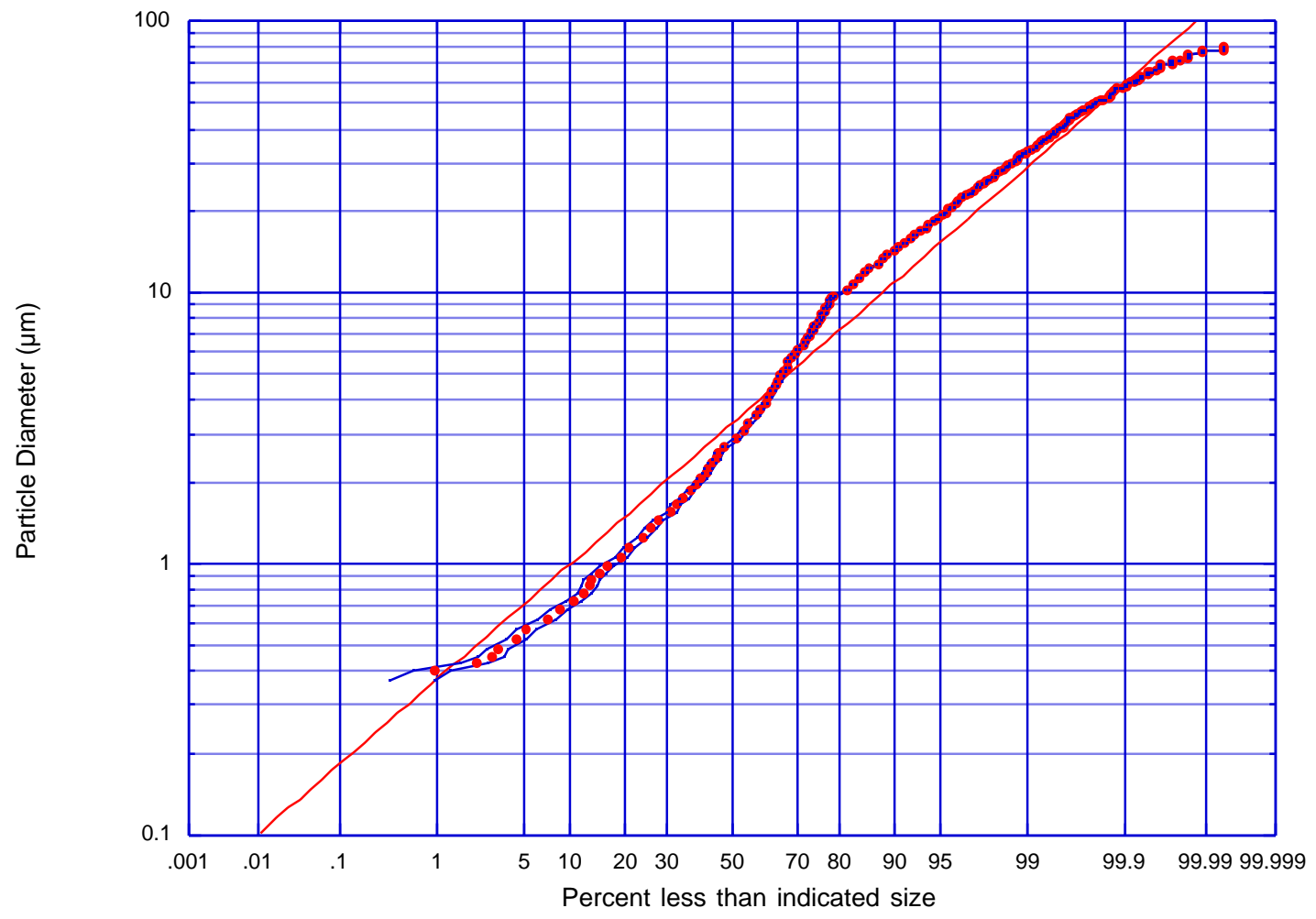

Analysis Summary Table:

\begin{tabular}{|c|c|c|c|c|c|}
\hline \multicolumn{2}{|c|}{$\begin{array}{c}\text { magnification, } \\
\text { number of images }\end{array}$} & \multicolumn{2}{|c|}{ area analyzed $^{1}(\%)$} & counted size range $(\mu \mathrm{m})$ & \multirow{2}{*}{$\begin{array}{c}\begin{array}{c}\text { total number of } \\
\text { particles (not scaled) }\end{array} \\
5522\end{array}$} \\
\hline $50 x$ & 6 & \multicolumn{2}{|r|}{0.894} & $d_{p}>9.96$ & \\
\hline $100 x$ & 6 & \multicolumn{2}{|r|}{0.224} & $5.27<d_{p}<9.96$ & 3085 \\
\hline $200 x$ & 6 & \multicolumn{2}{|r|}{0.0558} & $2.68<d_{p}<5.27$ & 2125 \\
\hline $500 x$ & 6 & \multicolumn{2}{|r|}{0.00893} & $1.56<\mathrm{d}_{\mathrm{p}}<2.68$ & 1016 \\
\hline 1000x, & 6 & \multicolumn{2}{|r|}{0.00217} & $0.00<\mathrm{d}_{\mathrm{p}}<1.56$ & 411 \\
\hline \multicolumn{2}{|c|}{ Totals: } & \multicolumn{2}{|r|}{$1.18 \%$} & - & 12159 \\
\hline \multicolumn{4}{|c|}{ data moments ${ }^{2}:$} & \multicolumn{2}{|c|}{ log-normal fit moments ${ }^{2}$ : } \\
\hline \multicolumn{3}{|c|}{ GMD $(\mu \mathrm{m}):$} & 3.19 & GMD $(\mu \mathrm{m}):$ & 3.30 \\
\hline \multicolumn{2}{|c|}{ GSD: } & & 3.04 & GSD: & 2.55 \\
\hline \multicolumn{3}{|c|}{ lower $95 \%(\mu \mathrm{m})$ : } & 0.35 & $\mathrm{R}^{2}:$ & 0.9805 \\
\hline \multicolumn{3}{|c|}{ upper $95 \%(\mu \mathrm{m})$ : } & 29.40 & lower $95 \%(\mu \mathrm{m})$ : & 0.51 \\
\hline & & & & upper $95 \%(\mu \mathrm{m})$ : & 21.47 \\
\hline
\end{tabular}

1. based on overall filter area of $1.59 \times 10^{9} \mu \mathrm{m}^{2}$

2. $\mathrm{GMD}=$ geometric mean diameter, $\mathrm{GSD}=$ geometric standard deviation, $\mathrm{R}=$ linear correlation coefficient

Figure 3.33 Module III, Sample 27 (TSFH09-02) count-based particle size distribution. 


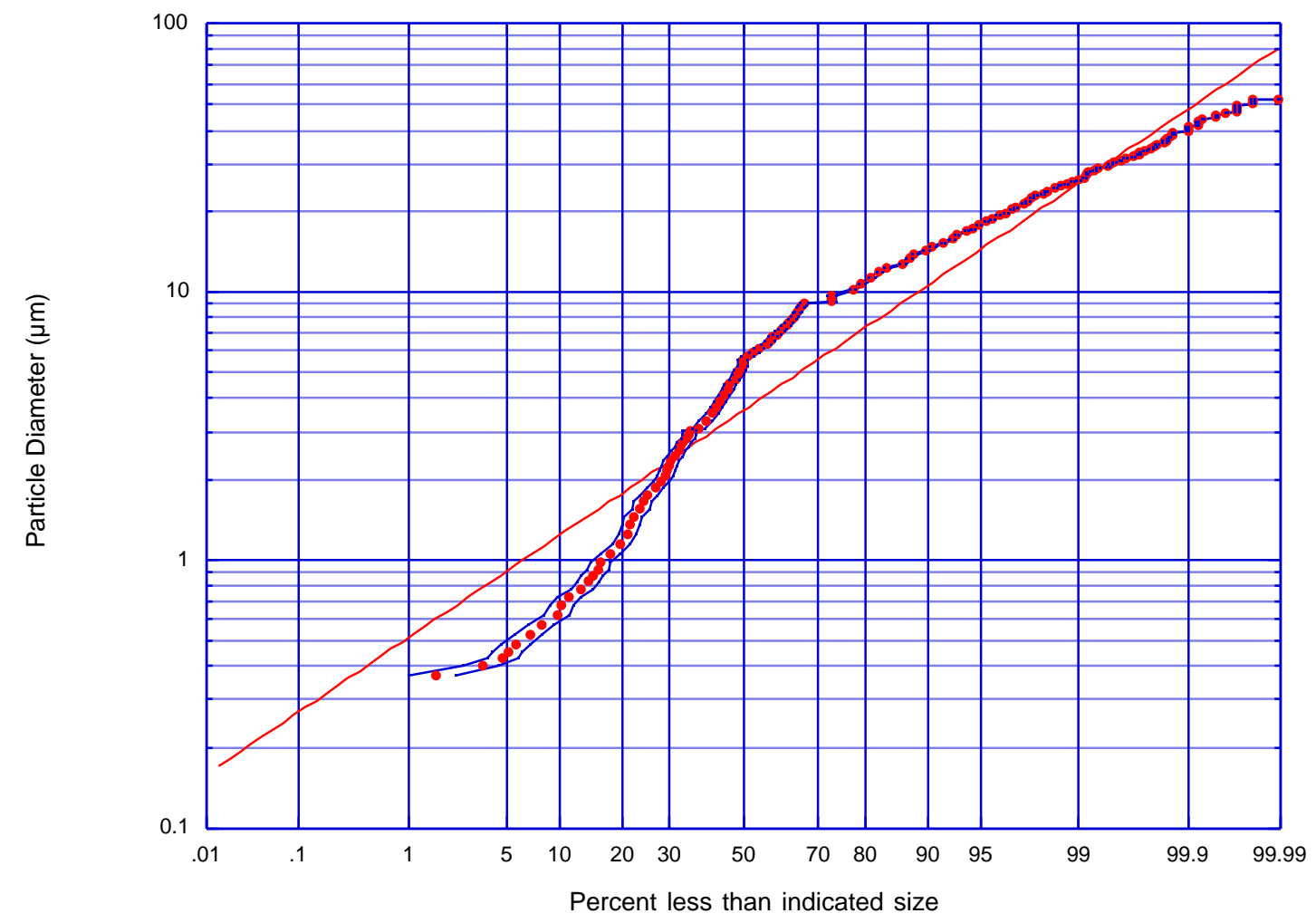

Analysis Summary Table:

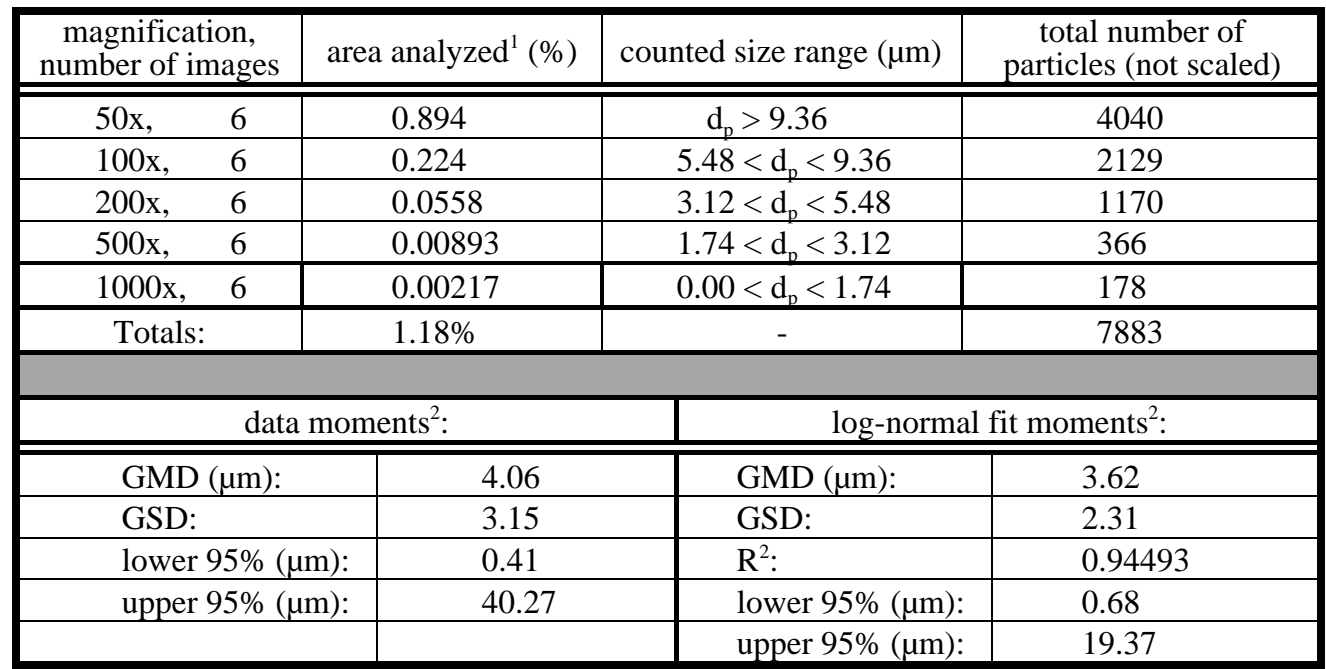

1. based on overall filter area of $1.59 \times 10^{9} \mu \mathrm{m}^{2}$

2. $\mathrm{GMD}=$ geometric mean diameter, $\mathrm{GSD}=$ geometric standard deviation, $\mathrm{R}=$ linear correlation coefficient

Figure 3.34 Module III, Sample 28 (TSFH07-02) count-based particle size distribution. 


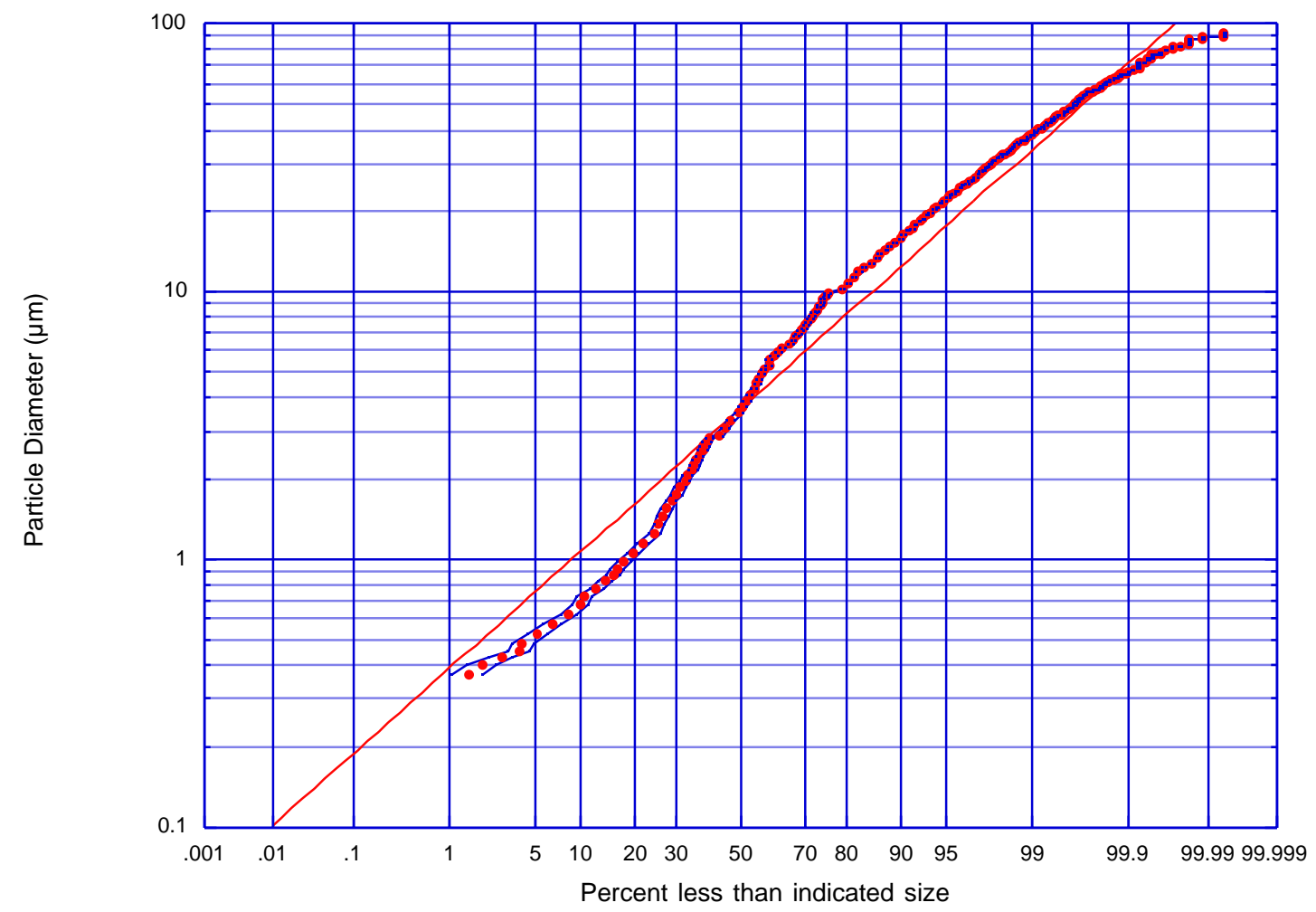

Analysis Summary Table:

\begin{tabular}{|c|c|c|c|c|c|}
\hline \multicolumn{2}{|c|}{$\begin{array}{c}\text { magnification, } \\
\text { number of images }\end{array}$} & \multicolumn{2}{|c|}{ area analyzed $^{1}(\%)$} & counted size range $(\mu \mathrm{m})$ & $\begin{array}{c}\text { total number of } \\
\text { particles (not scaled) }\end{array}$ \\
\hline $50 x$ & 6 & \multicolumn{2}{|r|}{0.894} & $d_{p}>10.10$ & 5991 \\
\hline $100 x$ & 6 & \multicolumn{2}{|r|}{0.224} & $5.37<\mathrm{d}_{\mathrm{p}}<10.10$ & 4056 \\
\hline $200 x$ & 6 & \multicolumn{2}{|r|}{0.0558} & $2.90<\mathrm{d}_{\mathrm{p}}<5.37$ & 1880 \\
\hline $500 x$ & 6 & \multicolumn{2}{|r|}{0.00893} & $1.64<\mathrm{d}_{\mathrm{p}}<2.90$ & 746 \\
\hline 1000x, & 6 & \multicolumn{2}{|r|}{0.00217} & $0.00<\mathrm{d}_{\mathrm{p}}<1.64$ & 357 \\
\hline \multicolumn{2}{|c|}{ Totals: } & \multicolumn{2}{|r|}{$1.18 \%$} & - & 13030 \\
\hline \multicolumn{4}{|c|}{ data moments ${ }^{2}:$} & \multicolumn{2}{|c|}{ log-normal fit moments ${ }^{2}$ : } \\
\hline \multicolumn{3}{|c|}{ GMD $(\mu \mathrm{m}):$} & 3.65 & GMD $(\mu \mathrm{m}):$ & 3.65 \\
\hline \multicolumn{2}{|c|}{ GSD: } & & 3.23 & GSD: & 2.61 \\
\hline \multicolumn{3}{|c|}{ lower $95 \%(\mu \mathrm{m})$ : } & 0.35 & $\mathrm{R}^{2}$ & 0.97837 \\
\hline \multirow{2}{*}{\multicolumn{3}{|c|}{ upper $95 \%(\mu \mathrm{m})$ : }} & 38.05 & lower $95 \%(\mu \mathrm{m})$ : & 0.53 \\
\hline & & & & upper $95 \%(\mu \mathrm{m})$ : & 24.86 \\
\hline
\end{tabular}

1. based on overall filter area of $1.59 \times 10^{9} \mu \mathrm{m}^{2}$

2. $\mathrm{GMD}=$ geometric mean diameter, $\mathrm{GSD}=$ geometric standard deviation, $\mathrm{R}=$ linear correlation coefficient

Figure 3.35 Module III, Sample 29 (TSFH22-02) count-based particle size distribution. 


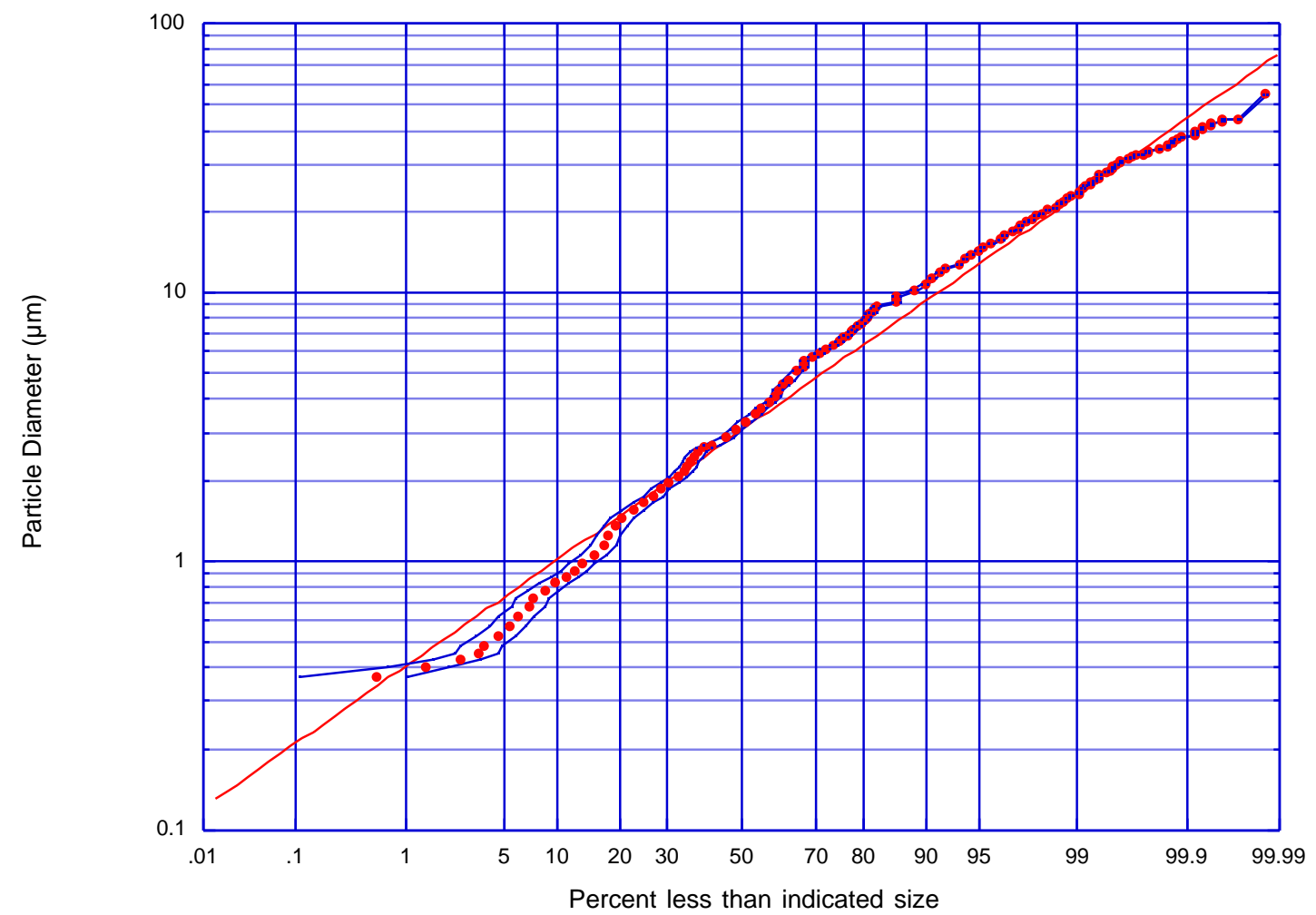

Analysis Summary Table:

\begin{tabular}{|c|c|c|c|c|c|}
\hline \multicolumn{2}{|c|}{$\begin{array}{c}\text { magnification, } \\
\text { number of images }\end{array}$} & \multicolumn{2}{|c|}{ area analyzed $^{1}(\%)$} & counted size range $(\mu \mathrm{m})$ & $\begin{array}{c}\text { total number of } \\
\text { particles (not scaled) }\end{array}$ \\
\hline $50 x$ & 6 & \multicolumn{2}{|r|}{0.894} & $d_{p}>8.94$ & 1532 \\
\hline $100 x$ & 6 & \multicolumn{2}{|r|}{0.224} & $4.92<\mathrm{d}_{\mathrm{p}}<8.94$ & 1583 \\
\hline $200 x$ & 6 & \multicolumn{2}{|r|}{0.0558} & $2.78<\mathrm{d}_{\mathrm{p}}<4.92$ & 905 \\
\hline $500 x$ & 6 & \multicolumn{2}{|r|}{0.00893} & $1.60<d_{p}<2.78$ & 436 \\
\hline 1000x, & 6 & \multicolumn{2}{|r|}{0.00217} & $0.00<\mathrm{d}_{\mathrm{p}}<1.60$ & 124 \\
\hline \multicolumn{2}{|c|}{ Totals: } & \multicolumn{2}{|r|}{$1.18 \%$} & - & 4580 \\
\hline \multicolumn{4}{|c|}{ data moments ${ }^{2}:$} & \multicolumn{2}{|c|}{ log-normal fit moments ${ }^{2}$ : } \\
\hline \multicolumn{3}{|c|}{ GMD $(\mu \mathrm{m}):$} & 3.28 & GMD $(\mu \mathrm{m}):$ & 3.06 \\
\hline \multicolumn{2}{|c|}{ GSD: } & & 2.63 & GSD: & 2.38 \\
\hline \multicolumn{3}{|c|}{ lower $95 \%(\mu \mathrm{m})$ : } & 0.47 & $\mathrm{R}^{2}$ & 0.98856 \\
\hline \multirow{2}{*}{\multicolumn{3}{|c|}{ upper $95 \%(\mu \mathrm{m})$ : }} & 22.72 & lower $95 \%(\mu \mathrm{m})$ : & 0.54 \\
\hline & & & & upper $95 \%(\mu \mathrm{m})$ : & 17.40 \\
\hline
\end{tabular}

1. based on overall filter area of $1.59 \times 10^{9} \mu \mathrm{m}^{2}$

2. $\mathrm{GMD}=$ geometric mean diameter, $\mathrm{GSD}=$ geometric standard deviation, $\mathrm{R}=$ linear correlation coefficient

Figure 3.36 Module III, Sample 30 (TSFH25-02) count-based particle size distribution. 


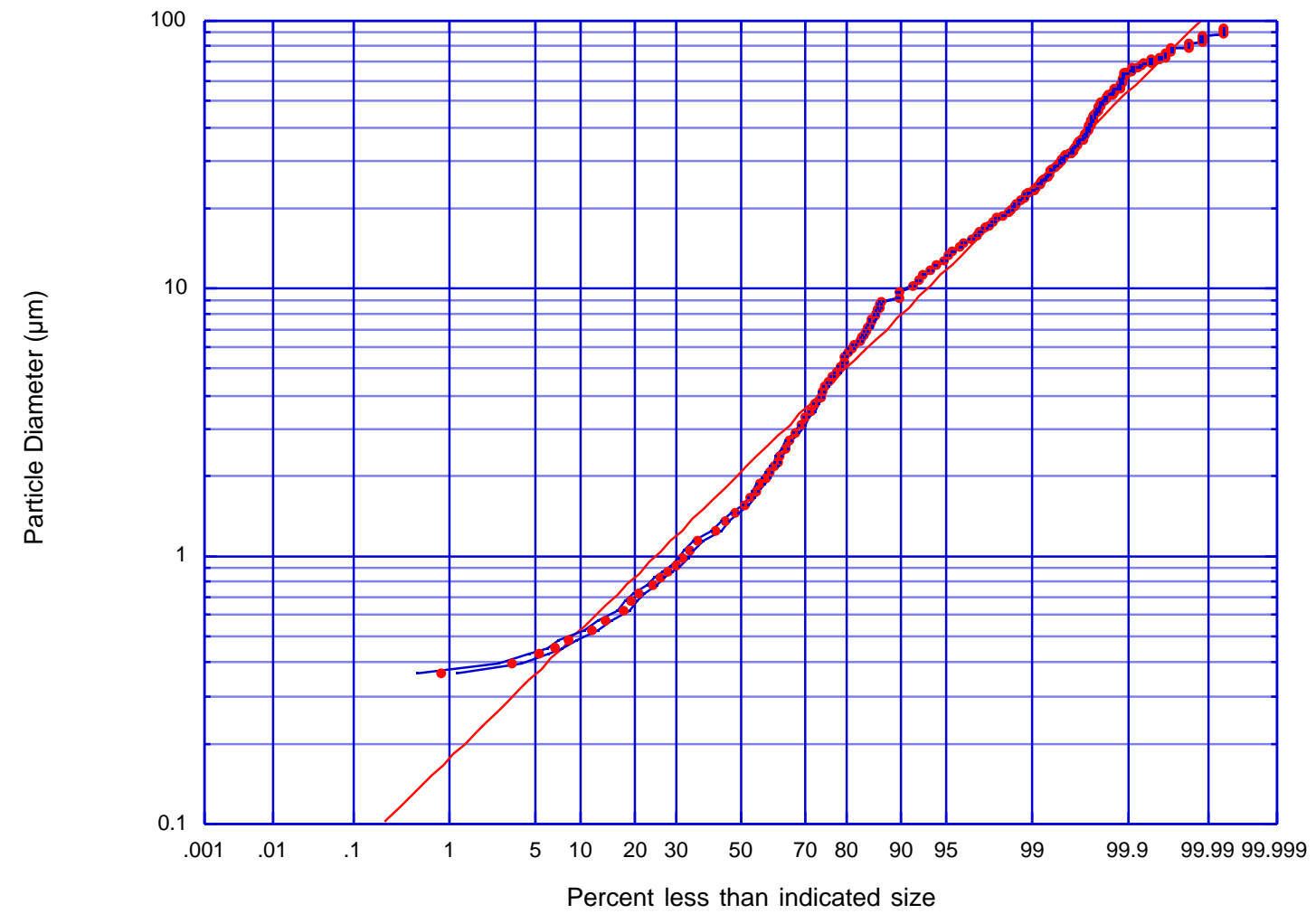

Analysis Summary Table:

\begin{tabular}{|c|c|c|c|c|c|}
\hline \multicolumn{2}{|c|}{$\begin{array}{c}\text { magnification, } \\
\text { number of images }\end{array}$} & \multicolumn{2}{|c|}{ area analyzed $^{1}(\%)$} & counted size range $(\mu \mathrm{m})$ & $\begin{array}{c}\text { total number of } \\
\text { particles (not scaled) }\end{array}$ \\
\hline $50 x$ & 6 & \multicolumn{2}{|r|}{0.894} & $d_{p}>9.14$ & 2772 \\
\hline $100 x$ & 6 & \multicolumn{2}{|r|}{0.224} & $4.87<d_{p}<9.14$ & 1781 \\
\hline $200 x$ & 6 & \multicolumn{2}{|r|}{0.0558} & $2.43<d_{p}<4.87$ & 1371 \\
\hline $500 x$ & 6 & \multicolumn{2}{|r|}{0.00893} & $1.25<\mathrm{d}_{\mathrm{p}}<2.43$ & 1037 \\
\hline 1000x, & 6 & \multicolumn{2}{|r|}{0.00217} & $0.00<\mathrm{d}_{\mathrm{p}}<1.25$ & 465 \\
\hline \multicolumn{2}{|c|}{ Totals: } & \multicolumn{2}{|r|}{$1.18 \%$} & - & 7426 \\
\hline \multicolumn{4}{|c|}{ data moments ${ }^{2}:$} & \multicolumn{2}{|c|}{ log-normal fit moments ${ }^{2}$ : } \\
\hline \multicolumn{3}{|c|}{ GMD $(\mu \mathrm{m}):$} & 1.96 & GMD $(\mu \mathrm{m}):$ & 2.06 \\
\hline \multicolumn{2}{|c|}{ GSD: } & & 2.94 & GSD: & 2.89 \\
\hline \multicolumn{3}{|c|}{ lower $95 \%(\mu \mathrm{m})$ : } & 0.23 & $\mathrm{R}^{2}$ & 0.98894 \\
\hline \multirow{2}{*}{\multicolumn{3}{|c|}{ upper $95 \%(\mu \mathrm{m})$ : }} & 16.98 & lower $95 \%(\mu \mathrm{m})$ : & 0.25 \\
\hline & & & & upper $95 \%(\mu \mathrm{m})$ : & 17.18 \\
\hline
\end{tabular}

1. based on overall filter area of $1.59 \times 10^{9} \mu \mathrm{m}^{2}$

2. $\mathrm{GMD}=$ geometric mean diameter, $\mathrm{GSD}=$ geometric standard deviation, $\mathrm{R}=$ linear correlation coefficient

Figure 3.37 Module III, Sample 30A (TSFH24-02) count-based particle size distribution. 


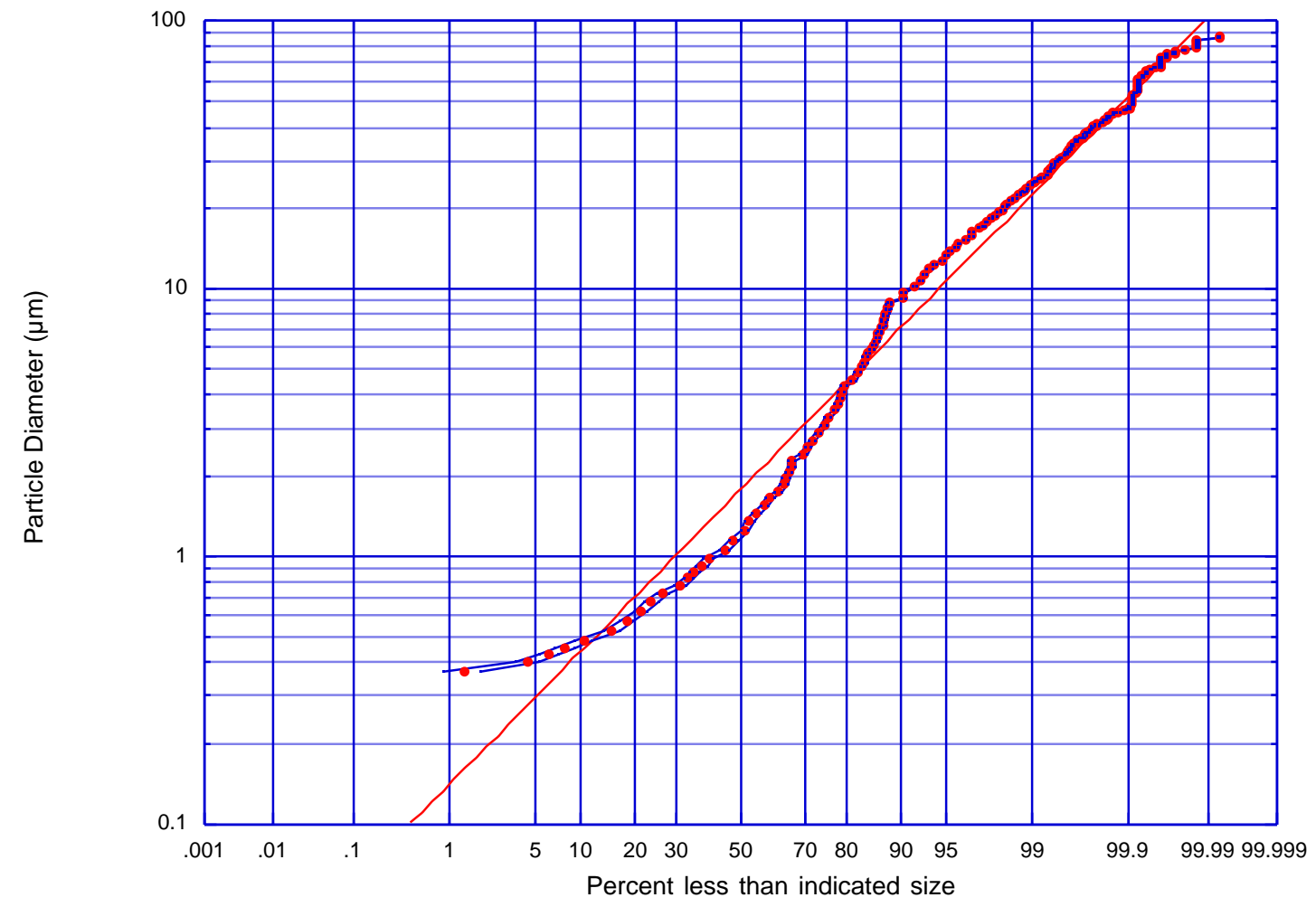

Analysis Summary Table:

\begin{tabular}{|c|c|c|c|c|c|}
\hline \multicolumn{2}{|c|}{$\begin{array}{c}\text { magnification, } \\
\text { number of images }\end{array}$} & \multicolumn{2}{|c|}{ area analyzed $^{1}(\%)$} & counted size range $(\mu \mathrm{m})$ & $\begin{array}{c}\text { total number of } \\
\text { particles (not scaled) }\end{array}$ \\
\hline $50 x$ & 6 & \multicolumn{2}{|r|}{0.894} & $d_{p}>9.17$ & 2149 \\
\hline $100 x$ & 6 & \multicolumn{2}{|r|}{0.224} & $4.50<d_{p}<9.17$ & 1018 \\
\hline $200 x$ & 6 & \multicolumn{2}{|r|}{0.0558} & $2.20<d_{p}<4.50$ & 928 \\
\hline $500 x$ & 6 & \multicolumn{2}{|r|}{0.00893} & $1.13<\mathrm{d}_{\mathrm{p}}<2.20$ & 712 \\
\hline 1000x, & 6 & \multicolumn{2}{|r|}{0.00217} & $0.00<\mathrm{d}_{\mathrm{p}}<1.13$ & 460 \\
\hline \multicolumn{2}{|c|}{ Totals: } & \multicolumn{2}{|r|}{$1.18 \%$} & - & 5267 \\
\hline \multicolumn{4}{|c|}{ data moments ${ }^{2}:$} & \multicolumn{2}{|c|}{ log-normal fit moments ${ }^{2}$ : } \\
\hline \multicolumn{3}{|c|}{ GMD $(\mu \mathrm{m}):$} & 1.66 & GMD $(\mu \mathrm{m}):$ & 1.78 \\
\hline \multicolumn{2}{|c|}{ GSD: } & & 2.95 & GSD: & 2.98 \\
\hline \multicolumn{3}{|c|}{ lower $95 \%(\mu \mathrm{m})$ : } & 0.19 & $\mathrm{R}^{2}$ & 0.98736 \\
\hline \multirow{2}{*}{\multicolumn{3}{|c|}{ upper $95 \%(\mu \mathrm{m})$ : }} & 14.51 & lower $95 \%(\mu \mathrm{m})$ : & 0.20 \\
\hline & & & & upper $95 \%(\mu \mathrm{m})$ : & 15.82 \\
\hline
\end{tabular}

1. based on overall filter area of $1.59 \times 10^{9} \mu \mathrm{m}^{2}$

2. $\mathrm{GMD}=$ geometric mean diameter, $\mathrm{GSD}=$ geometric standard deviation, $\mathrm{R}=$ linear correlation coefficient

Figure 3.38 Module III, Sample 30B (TSFH21-02) count-based particle size distribution. 


\subsubsection{Module IV Results}

Figure 3.39 through Figure 3.50 display details of particle size distributions for corresponding collection locations of Module IV. Table 3.4 gives a summary of Module IV results.

Table 3.4 Summary of dust collected from locations in Tore Supra Module IV.

\begin{tabular}{|c|c|c|c|c|c|c|}
\hline \multirow{2}{*}{$\begin{array}{c}\text { Poloidal } \\
\text { Location }\end{array}$} & Sample & Filter ID & \multirow{2}{*}{$\begin{array}{c}\text { Sampled } \\
\text { Area }\left(\mathrm{cm}^{2}\right)\end{array}$} & $\begin{array}{c}\text { Collected } \\
\text { Mass }(\mathrm{mg})\end{array}$ & \multicolumn{2}{|c|}{$\begin{array}{c}\text { Count-based Size } \\
\text { Distribution Parameters }\end{array}$} \\
\hline \hline 1 & & & GMD $(\mu \mathrm{m})$ & GSD \\
\hline 2 & 31 & TSFH18-02 & 621.2 & 0.4 & 3.72 & 3.21 \\
\hline 4 & 33 & TSFH16-02 & 1,553 & 9.9 & 2.47 & 3.05 \\
\hline 3 & 34 & TSFH01-03 & 689.0 & 0.9 & 3.11 & 2.99 \\
\hline 6 & 35 & TSFH14-02 & 1,800 & 0.9 & 2.86 & 3.14 \\
\hline 5 & 36 & TSFH02-03 & 1,200 & 1.7 & 3.04 & 3.03 \\
\hline 7 & 37 & TSFH04-03 & 428.0 & 1.1 & 2.33 & 3.11 \\
\hline 8 & 38 & TSFH12-02 & 84.00 & 0.4 & 2.72 & 2.94 \\
\hline 10 & 39 & TSFH03-03 & 1,429 & 1.8 & 4.08 & 3.21 \\
\hline 9 & 40 & TSFH17-02 & 294.0 & 0.3 & 4.71 & 3.15 \\
\hline 11 & $40 \mathrm{~A}$ & TSFH19-02 & 160.0 & 31.9 & 1.54 & 2.67 \\
\hline 12 & $40 \mathrm{~B}$ & TSFH15-02 & 480.0 & 26.2 & 2.22 & 2.57 \\
\hline
\end{tabular}




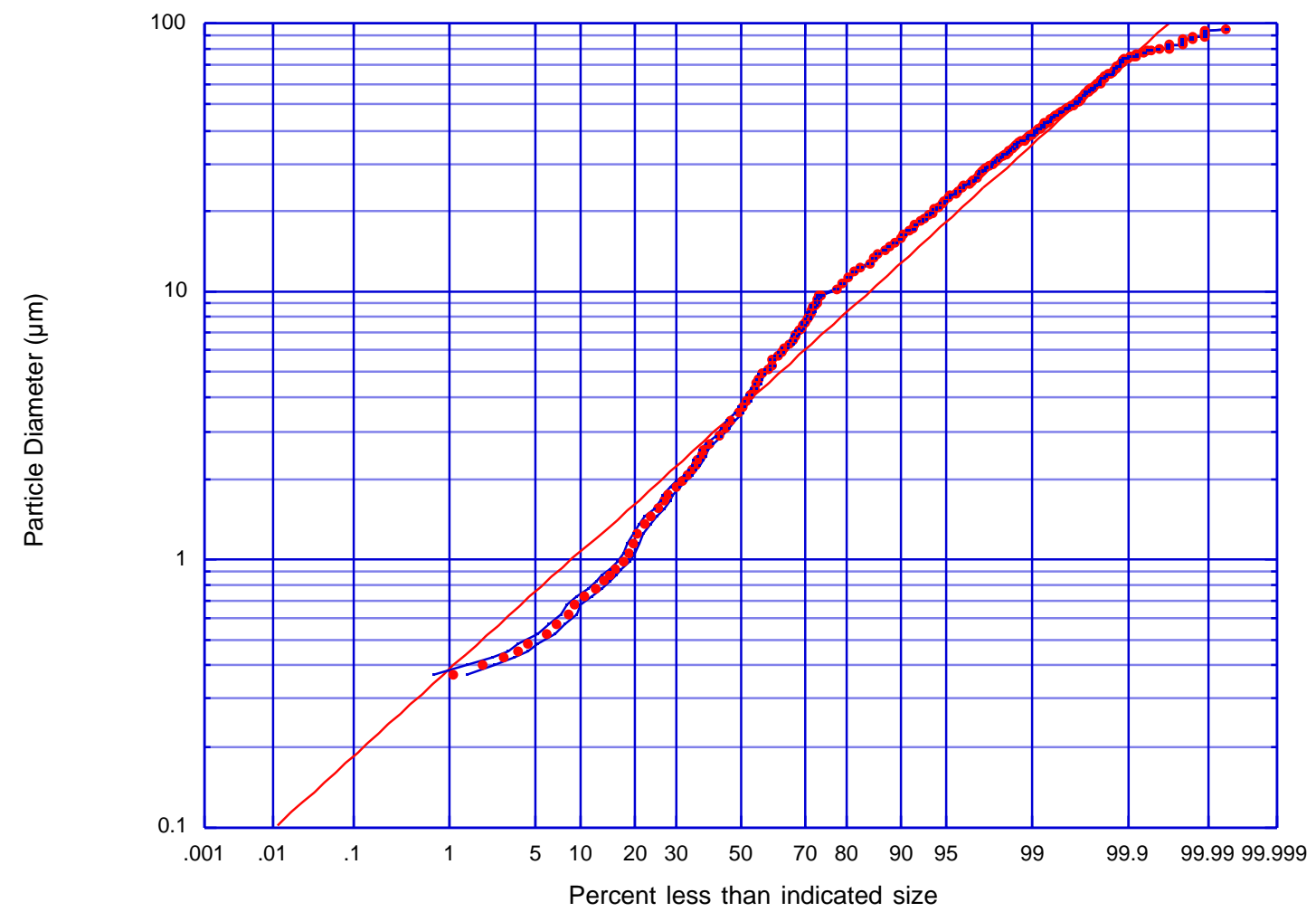

Analysis Summary Table:

\begin{tabular}{|c|c|c|c|c|c|}
\hline \multicolumn{2}{|c|}{$\begin{array}{c}\text { magnification, } \\
\text { number of images }\end{array}$} & \multicolumn{2}{|c|}{ area analyzed $^{1}(\%)$} & counted size range $(\mu \mathrm{m})$ & $\begin{array}{c}\text { total number of } \\
\text { particles (not scaled) }\end{array}$ \\
\hline $50 x$ & 6 & \multicolumn{2}{|r|}{0.894} & $d_{p}>9.79$ & 6984 \\
\hline $100 x$ & 6 & \multicolumn{2}{|r|}{0.224} & $4.97<d_{p}<9.79$ & 3567 \\
\hline $200 x$ & 6 & \multicolumn{2}{|r|}{0.0558} & $2.63<d_{p}<4.97$ & 2134 \\
\hline $500 x$ & 6 & \multicolumn{2}{|r|}{0.00893} & $1.49<\mathrm{d}_{\mathrm{p}}<2.63$ & 895 \\
\hline 1000x, & 6 & \multicolumn{2}{|r|}{0.00217} & $0.00<\mathrm{d}_{\mathrm{p}}<1.49$ & 320 \\
\hline \multicolumn{2}{|c|}{ Totals: } & \multicolumn{2}{|r|}{$1.18 \%$} & - & 13900 \\
\hline \multicolumn{4}{|c|}{ data moments ${ }^{2}:$} & \multicolumn{2}{|c|}{ log-normal fit moments ${ }^{2}$ : } \\
\hline \multicolumn{3}{|c|}{ GMD $(\mu \mathrm{m}):$} & 3.72 & GMD $(\mu \mathrm{m}):$ & 3.69 \\
\hline \multicolumn{2}{|c|}{ GSD: } & & 3.21 & GSD: & 2.64 \\
\hline \multicolumn{3}{|c|}{ lower $95 \%(\mu \mathrm{m})$ : } & 0.36 & $\mathrm{R}^{2}$ & 0.98074 \\
\hline \multirow{2}{*}{\multicolumn{3}{|c|}{ upper $95 \%(\mu \mathrm{m})$ : }} & 38.37 & lower $95 \%(\mu \mathrm{m})$ : & 0.53 \\
\hline & & & & upper $95 \%(\mu \mathrm{m})$ : & 25.74 \\
\hline
\end{tabular}

1. based on overall filter area of $1.59 \times 10^{9} \mu \mathrm{m}^{2}$

2. $\mathrm{GMD}=$ geometric mean diameter, $\mathrm{GSD}=$ geometric standard deviation, $\mathrm{R}=$ linear correlation coefficient

Figure 3.39 Module IV, Sample 31 (TSFH18-02) count-based particle size distribution. 


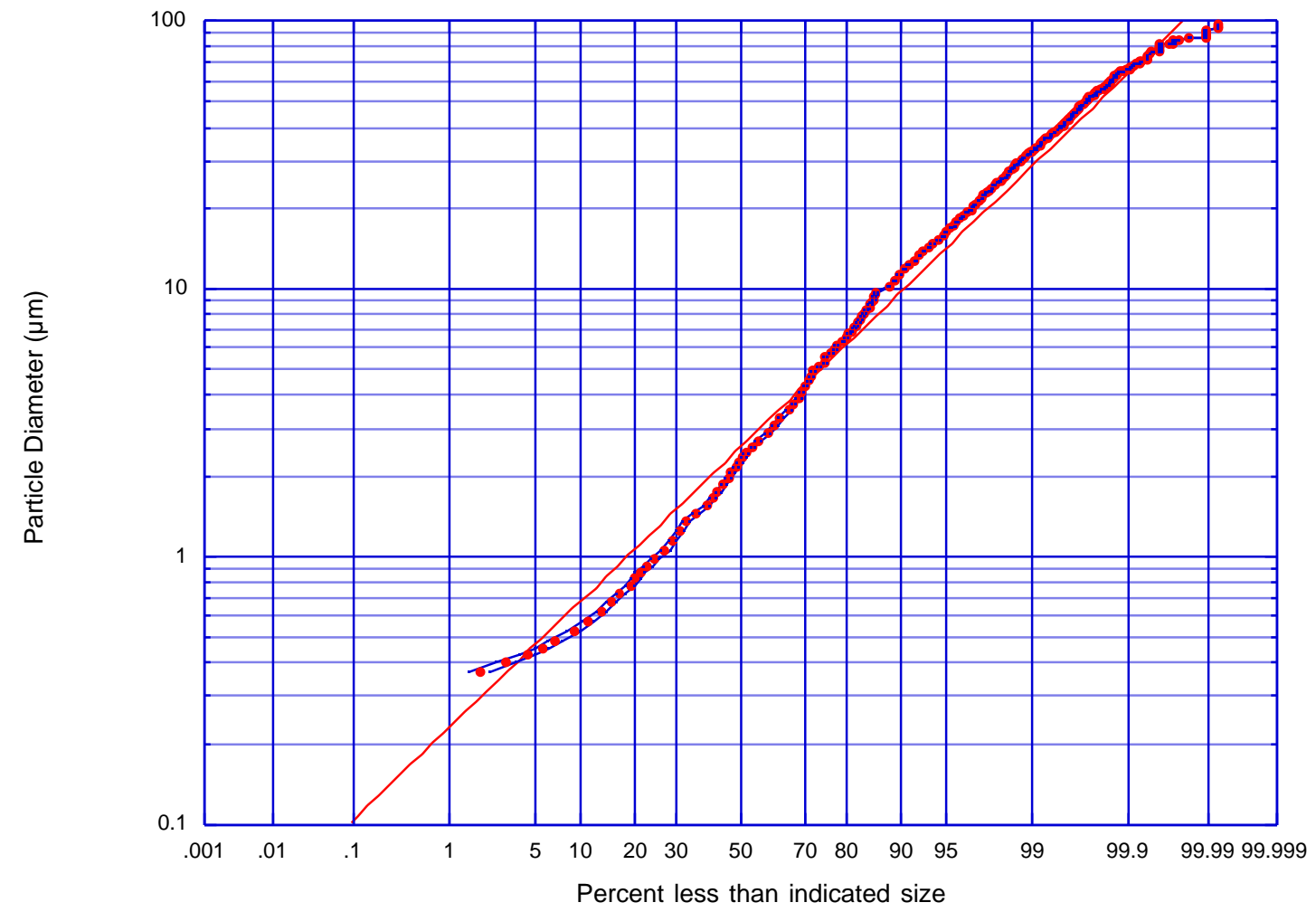

Analysis Summary Table:

\begin{tabular}{|c|c|c|c|c|c|}
\hline \multicolumn{2}{|c|}{$\begin{array}{c}\text { magnification, } \\
\text { number of images }\end{array}$} & \multicolumn{2}{|c|}{ area analyzed $^{1}(\%)$} & counted size range $(\mu \mathrm{m})$ & $\begin{array}{c}\text { total number of } \\
\text { particles (not scaled) }\end{array}$ \\
\hline $50 x$ & 6 & \multicolumn{2}{|r|}{0.894} & $d_{p}>9.75$ & 5942 \\
\hline $100 x$ & 6 & \multicolumn{2}{|r|}{0.224} & $5.03<d_{p}<9.75$ & 4424 \\
\hline $200 x$ & 6 & \multicolumn{2}{|r|}{0.0558} & $2.56<d_{p}<5.03$ & 3311 \\
\hline $500 x$ & 6 & \multicolumn{2}{|r|}{0.00893} & $1.41<\mathrm{d}_{\mathrm{p}}<2.56$ & 1613 \\
\hline 1000x, & 6 & \multicolumn{2}{|r|}{0.00217} & $0.00<\mathrm{d}_{\mathrm{p}}<1.41$ & 715 \\
\hline \multicolumn{2}{|c|}{ Totals: } & \multicolumn{2}{|r|}{$1.18 \%$} & - & 16005 \\
\hline \multicolumn{4}{|c|}{ data moments ${ }^{2}:$} & \multicolumn{2}{|c|}{ log-normal fit moments ${ }^{2}$ : } \\
\hline \multicolumn{3}{|c|}{ GMD $(\mu \mathrm{m}):$} & 2.47 & GMD $(\mu \mathrm{m}):$ & 2.58 \\
\hline \multicolumn{2}{|c|}{ GSD: } & & 3.05 & GSD: & 2.83 \\
\hline \multicolumn{3}{|c|}{ lower $95 \%(\mu \mathrm{m})$ : } & 0.27 & $\mathrm{R}^{2}$ & 0.98901 \\
\hline \multirow{2}{*}{\multicolumn{3}{|c|}{ upper $95 \%(\mu \mathrm{m})$ : }} & 22.91 & lower $95 \%(\mu \mathrm{m})$ : & 0.32 \\
\hline & & & & upper $95 \%(\mu \mathrm{m})$ : & 20.59 \\
\hline
\end{tabular}

1. based on overall filter area of $1.59 \times 10^{9} \mu \mathrm{m}^{2}$

2. $\mathrm{GMD}=$ geometric mean diameter, $\mathrm{GSD}=$ geometric standard deviation, $\mathrm{R}=$ linear correlation coefficient

Figure 3.40 Module IV, Sample 32 (TSFH16-02) count-based particle size distribution. 


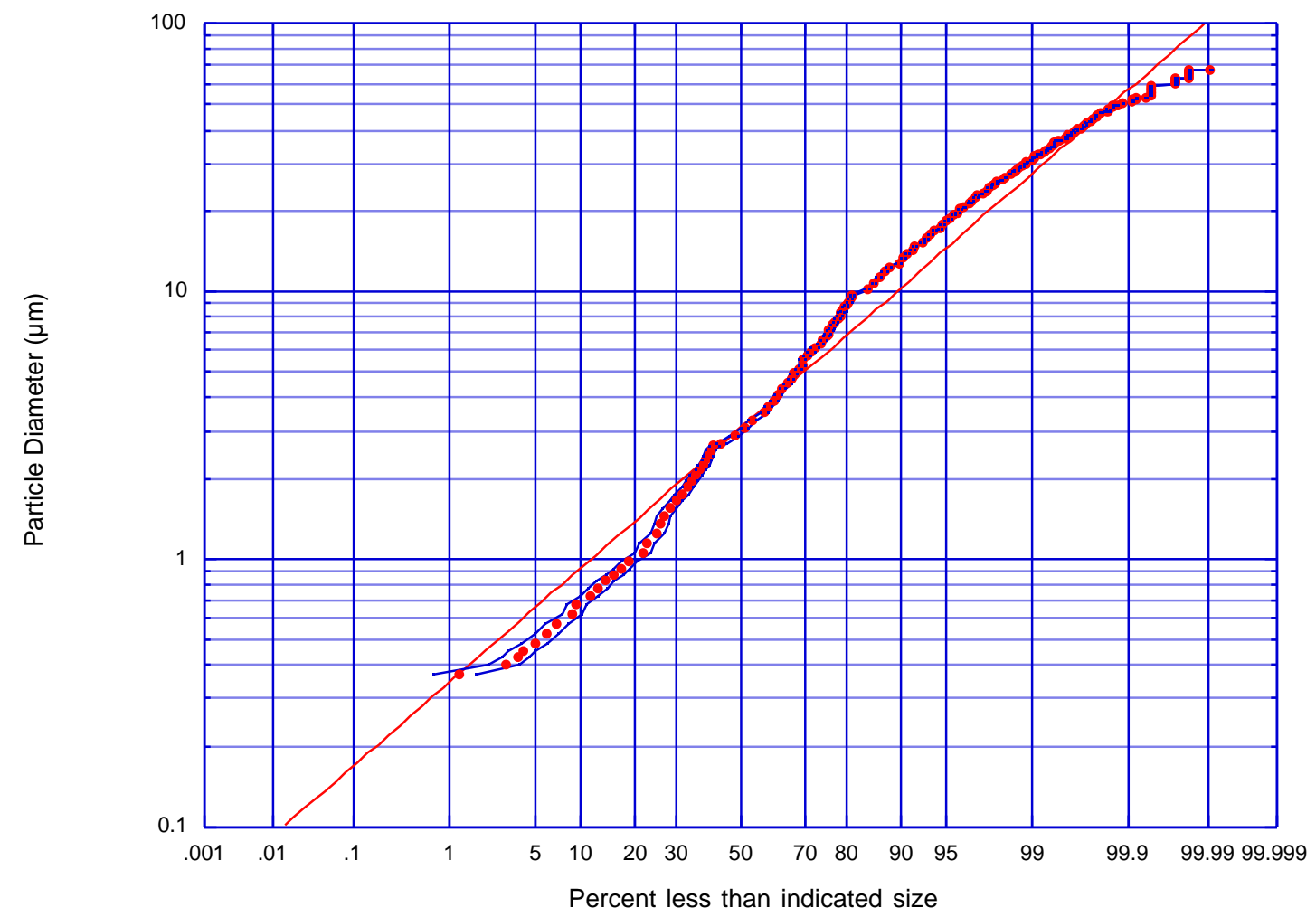

Analysis Summary Table:

\begin{tabular}{|c|c|c|c|c|c|}
\hline \multicolumn{2}{|c|}{$\begin{array}{c}\text { magnification, } \\
\text { number of images }\end{array}$} & \multicolumn{2}{|c|}{ area analyzed $^{1}(\%)$} & counted size range $(\mu \mathrm{m})$ & $\begin{array}{c}\text { total number of } \\
\text { particles (not scaled) }\end{array}$ \\
\hline $50 x$ & 6 & \multicolumn{2}{|r|}{0.894} & $d_{p}>9.75$ & 3102 \\
\hline $100 x$ & 6 & \multicolumn{2}{|r|}{0.224} & $5.24<d_{p}<9.75$ & 1989 \\
\hline $200 x$ & 6 & \multicolumn{2}{|r|}{0.0558} & $2.69<d_{p}<5.24$ & 1726 \\
\hline $500 x$ & 6 & \multicolumn{2}{|r|}{0.00893} & $1.62<d_{p}<2.69$ & 577 \\
\hline 1000x, & 6 & \multicolumn{2}{|r|}{0.00217} & $0.00<\mathrm{d}_{\mathrm{p}}<1.62$ & 275 \\
\hline \multicolumn{2}{|c|}{ Totals: } & \multicolumn{2}{|r|}{$1.18 \%$} & - & 7669 \\
\hline \multicolumn{4}{|c|}{ data moments ${ }^{2}:$} & \multicolumn{2}{|c|}{ log-normal fit moments ${ }^{2}$ : } \\
\hline \multicolumn{3}{|c|}{ GMD $(\mu \mathrm{m}):$} & 3.11 & GMD $(\mu \mathrm{m}):$ & 3.09 \\
\hline \multicolumn{2}{|c|}{ GSD: } & & 2.99 & GSD: & 2.57 \\
\hline \multicolumn{3}{|c|}{ lower $95 \%(\mu \mathrm{m})$ : } & 0.35 & $\mathrm{R}^{2}$ & 0.98152 \\
\hline \multirow{2}{*}{\multicolumn{3}{|c|}{ upper $95 \%(\mu \mathrm{m})$ : }} & 27.86 & lower $95 \%(\mu \mathrm{m})$ : & 0.47 \\
\hline & & & & upper $95 \%(\mu \mathrm{m})$ : & 20.35 \\
\hline
\end{tabular}

1. based on overall filter area of $1.59 \times 10^{9} \mu \mathrm{m}^{2}$

2. $\mathrm{GMD}=$ geometric mean diameter, $\mathrm{GSD}=$ geometric standard deviation, $\mathrm{R}=$ linear correlation coefficient

Figure 3.41 Module IV, Sample 33 (TSFH01-03) count-based particle size distribution. 


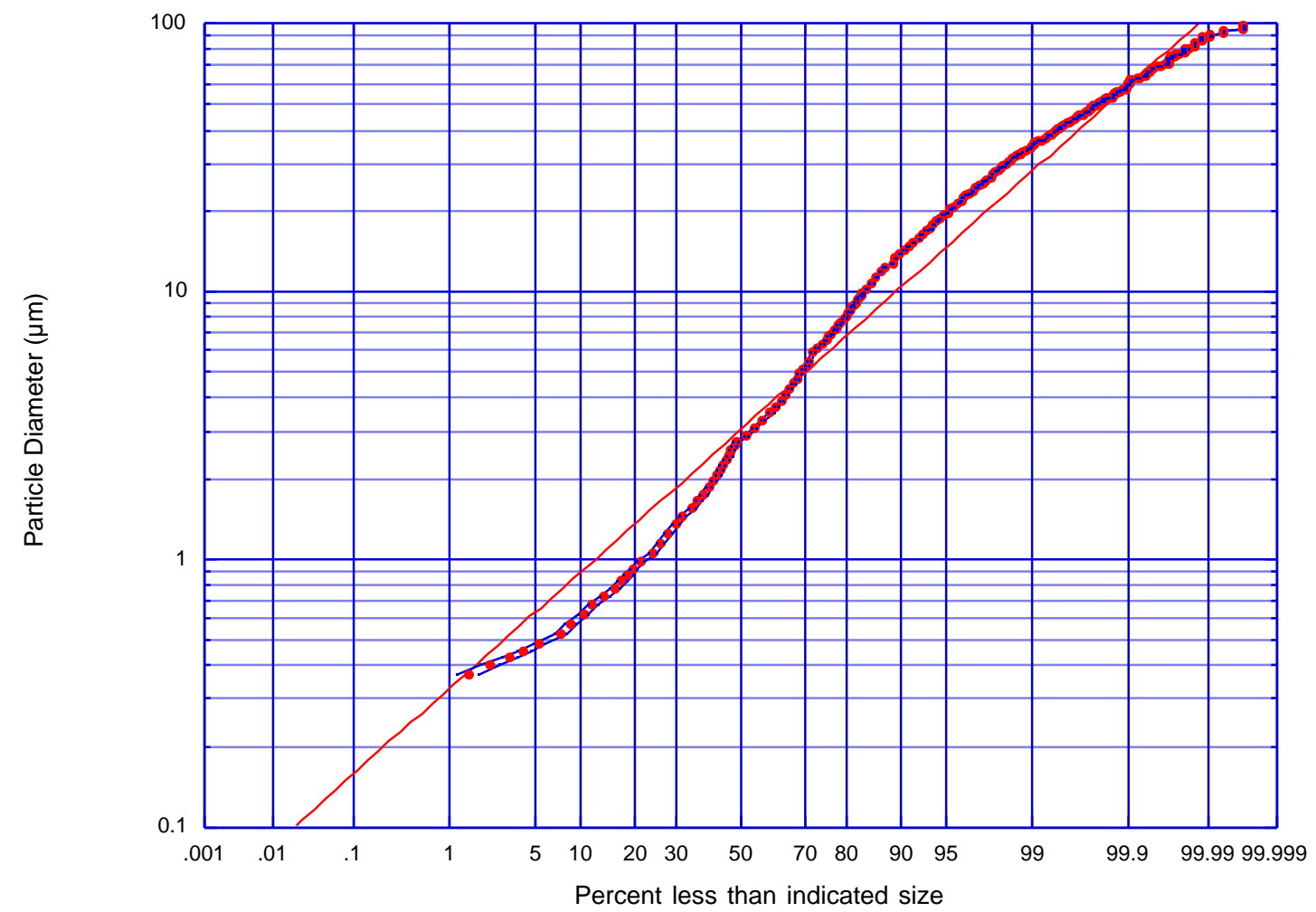

Analysis Summary Table:

\begin{tabular}{|c|c|c|c|c|c|}
\hline \multicolumn{2}{|c|}{$\begin{array}{c}\text { magnification, } \\
\text { number of images }\end{array}$} & \multicolumn{2}{|c|}{ area analyzed $^{1}(\%)$} & counted size range $(\mu \mathrm{m})$ & $\begin{array}{c}\text { total number of } \\
\text { particles (not scaled) }\end{array}$ \\
\hline $50 x$ & 6 & \multicolumn{2}{|r|}{0.894} & $d_{p}>10.51$ & 8397 \\
\hline $100 x$ & 6 & \multicolumn{2}{|r|}{0.224} & $5.58<\mathrm{d}_{\mathrm{p}}<10.51$ & 6469 \\
\hline $200 x$ & 6 & \multicolumn{2}{|r|}{0.0558} & $2.82<\mathrm{d}_{\mathrm{p}}<5.58$ & 4356 \\
\hline $500 x$ & 6 & \multicolumn{2}{|r|}{0.00893} & $1.58<\mathrm{d}_{\mathrm{p}}<2.82$ & 1788 \\
\hline 1000x, & 6 & \multicolumn{2}{|r|}{0.00217} & $0.00<\mathrm{d}_{\mathrm{p}}<1.58$ & 867 \\
\hline \multicolumn{2}{|c|}{ Totals: } & \multicolumn{2}{|r|}{$1.18 \%$} & - & 21877 \\
\hline \multicolumn{4}{|c|}{ data moments ${ }^{2}:$} & \multicolumn{2}{|c|}{ log-normal fit moments ${ }^{2}$ : } \\
\hline \multicolumn{3}{|c|}{ GMD $(\mu \mathrm{m}):$} & 2.86 & GMD $(\mu \mathrm{m}):$ & 3.05 \\
\hline \multicolumn{2}{|c|}{ GSD: } & & 3.14 & GSD: & 2.61 \\
\hline \multicolumn{3}{|c|}{ lower $95 \%(\mu \mathrm{m})$ : } & 0.29 & $\mathrm{R}^{2}$ & 0.98024 \\
\hline \multirow{2}{*}{\multicolumn{3}{|c|}{ upper $95 \%(\mu \mathrm{m})$ : }} & 28.15 & lower $95 \%(\mu \mathrm{m})$ : & 0.45 \\
\hline & & & & upper $95 \%(\mu \mathrm{m})$ : & 20.76 \\
\hline
\end{tabular}

1. based on overall filter area of $1.59 \times 10^{9} \mu \mathrm{m}^{2}$

2. $\mathrm{GMD}=$ geometric mean diameter, $\mathrm{GSD}=$ geometric standard deviation, $\mathrm{R}=$ linear correlation coefficient

Figure 3.42 Module IV, Sample 34 (TSFH14-02) count-based particle size distribution. 


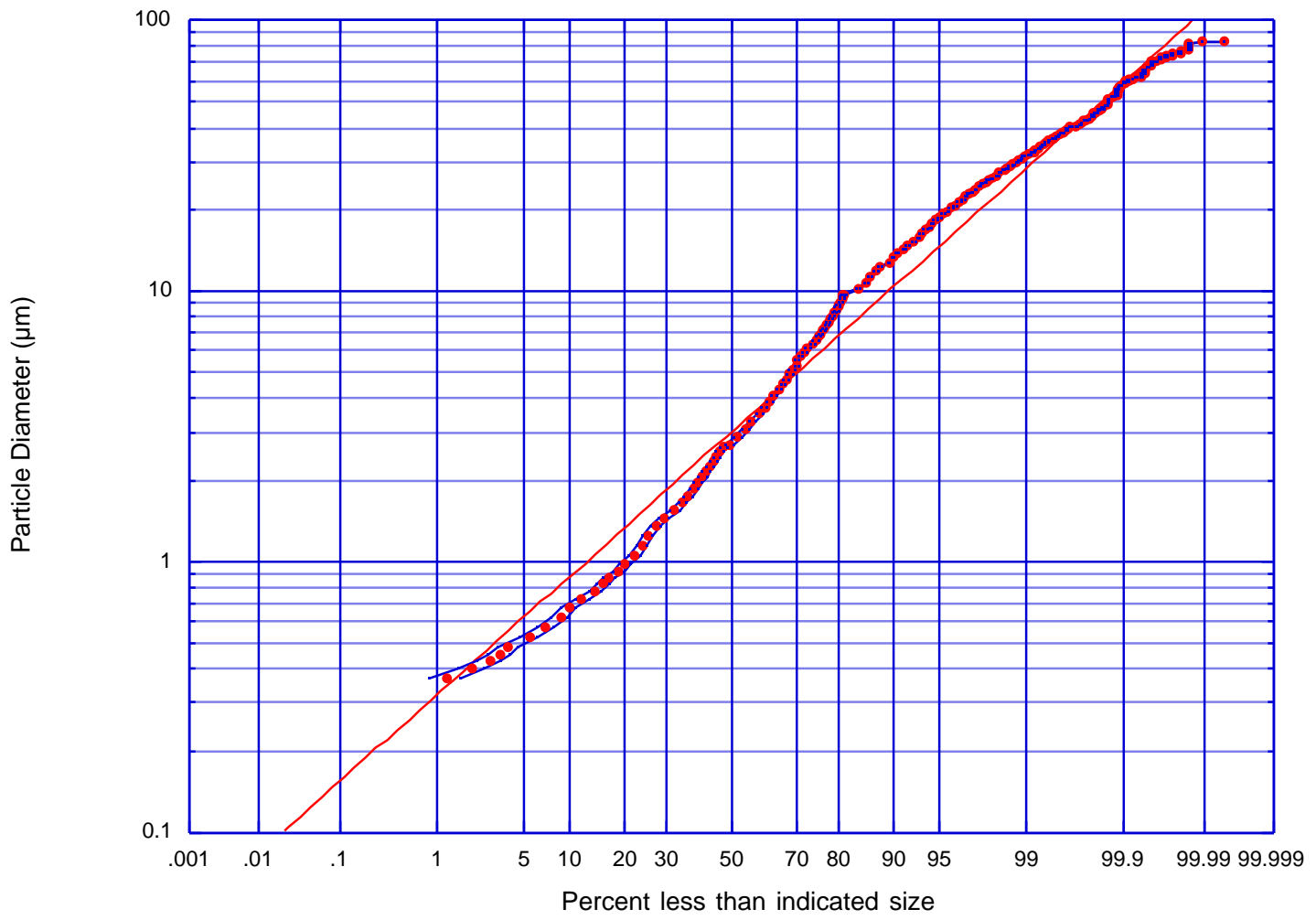

Analysis Summary Table:

\begin{tabular}{|c|c|c|c|c|c|}
\hline \multicolumn{2}{|c|}{$\begin{array}{c}\text { magnification, } \\
\text { number of images }\end{array}$} & \multicolumn{2}{|c|}{ area analyzed $^{1}(\%)$} & counted size range $(\mu \mathrm{m})$ & $\begin{array}{c}\text { total number of } \\
\text { particles (not scaled) }\end{array}$ \\
\hline $50 x$ & 6 & \multicolumn{2}{|r|}{0.894} & $d_{p}>9.91$ & 5139 \\
\hline $100 x$ & 6 & \multicolumn{2}{|r|}{0.224} & $5.25<d_{p}<9.91$ & 3229 \\
\hline $200 x$ & 6 & \multicolumn{2}{|r|}{0.0558} & $2.71<d_{p}<5.25$ & 2334 \\
\hline $500 x$ & 6 & \multicolumn{2}{|r|}{0.00893} & $1.59<\mathrm{d}_{\mathrm{p}}<2.71$ & 1035 \\
\hline 1000x, & 6 & \multicolumn{2}{|r|}{0.00217} & $0.00<\mathrm{d}_{\mathrm{p}}<1.59$ & 480 \\
\hline \multicolumn{2}{|c|}{ Totals: } & \multicolumn{2}{|r|}{$1.18 \%$} & - & 12217 \\
\hline \multicolumn{4}{|c|}{ data moments ${ }^{2}:$} & \multicolumn{2}{|c|}{ log-normal fit moments ${ }^{2}$ : } \\
\hline \multicolumn{3}{|c|}{ GMD $(\mu \mathrm{m}):$} & 2.96 & GMD $(\mu \mathrm{m}):$ & 3.03 \\
\hline \multicolumn{2}{|c|}{ GSD: } & & 3.03 & GSD: & 2.62 \\
\hline \multicolumn{3}{|c|}{ lower $95 \%(\mu \mathrm{m})$ : } & 0.32 & $\mathrm{R}^{2}$ & 0.98719 \\
\hline \multirow{2}{*}{\multicolumn{3}{|c|}{ upper $95 \%(\mu \mathrm{m})$ : }} & 27.27 & lower $95 \%(\mu \mathrm{m})$ : & 0.44 \\
\hline & & & & upper $95 \%(\mu \mathrm{m})$ : & 20.79 \\
\hline
\end{tabular}

1. based on overall filter area of $1.59 \times 10^{9} \mu \mathrm{m}^{2}$

2. $\mathrm{GMD}=$ geometric mean diameter, $\mathrm{GSD}=$ geometric standard deviation, $\mathrm{R}=$ linear correlation coefficient

Figure 3.43 Module IV, Sample 35 (TSFH13-02) count-based particle size distribution. 


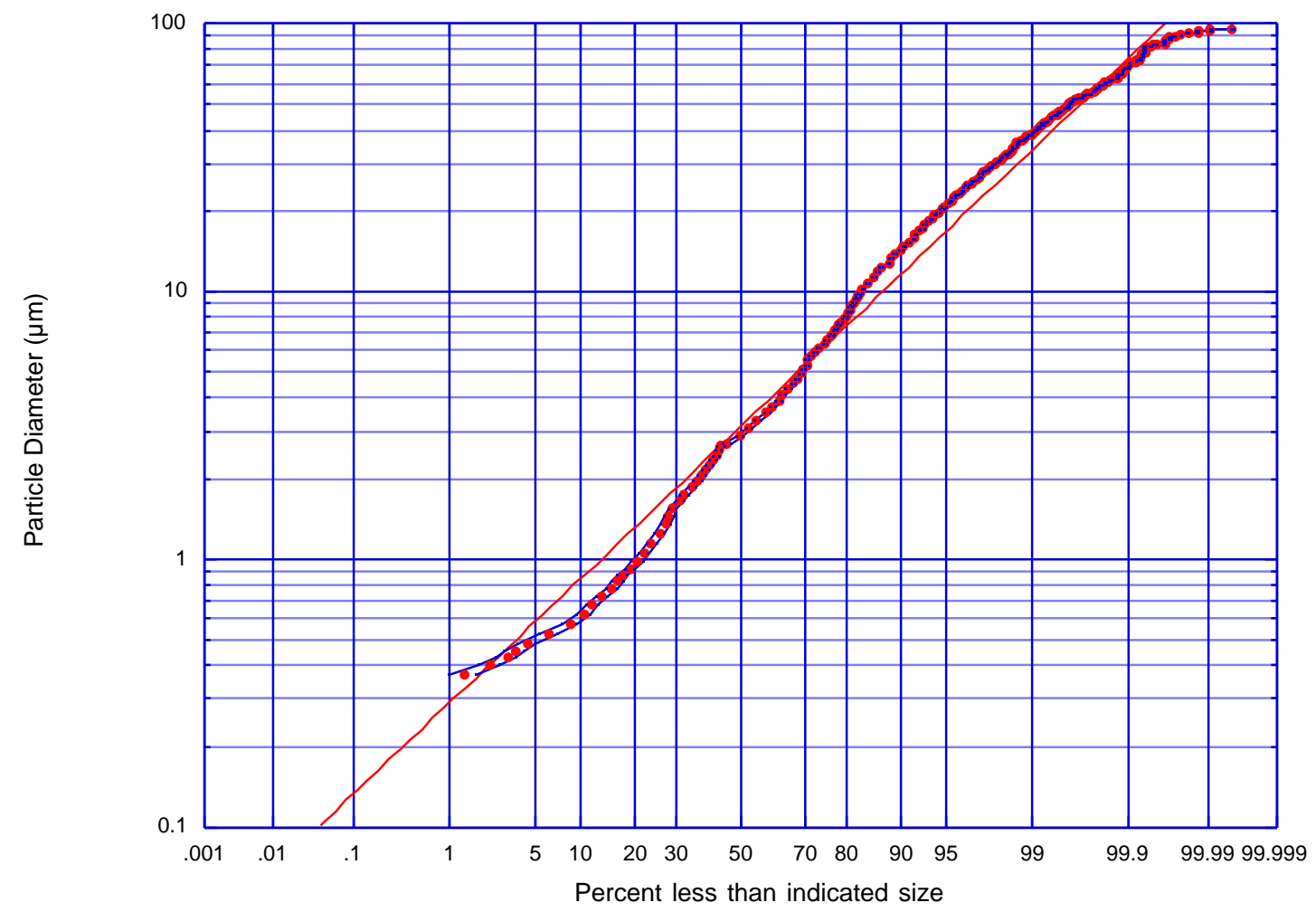

Analysis Summary Table:

\begin{tabular}{|c|c|c|c|c|c|}
\hline \multicolumn{2}{|c|}{$\begin{array}{c}\text { magnification, } \\
\text { number of images }\end{array}$} & \multicolumn{2}{|c|}{ area analyzed $^{1}(\%)$} & counted size range $(\mu \mathrm{m})$ & $\begin{array}{c}\text { total number of } \\
\text { particles (not scaled) }\end{array}$ \\
\hline $50 x$ & 6 & \multicolumn{2}{|r|}{0.894} & $d_{p}>10.44$ & 5837 \\
\hline $100 x$ & 6 & \multicolumn{2}{|r|}{0.224} & $5.38<\mathrm{d}_{\mathrm{p}}<10.44$ & 3956 \\
\hline $200 x$ & 6 & \multicolumn{2}{|r|}{0.0558} & $2.73<\mathrm{d}_{\mathrm{p}}<5.38$ & 3183 \\
\hline $500 x$ & 6 & \multicolumn{2}{|r|}{0.00893} & $1.57<\mathrm{d}_{\mathrm{p}}<2.73$ & 1218 \\
\hline 1000x, & 6 & \multicolumn{2}{|r|}{0.00217} & $0.00<\mathrm{d}_{\mathrm{p}}<1.57$ & 497 \\
\hline \multicolumn{2}{|c|}{ Totals: } & \multicolumn{2}{|r|}{$1.18 \%$} & - & 14691 \\
\hline \multicolumn{4}{|c|}{ data moments ${ }^{2}:$} & \multicolumn{2}{|c|}{ log-normal fit moments ${ }^{2}$ : } \\
\hline \multicolumn{3}{|c|}{ GMD $(\mu \mathrm{m}):$} & 3.04 & GMD $(\mu \mathrm{m}):$ & 3.14 \\
\hline \multicolumn{2}{|c|}{ GSD: } & & 3.11 & GSD: & 2.78 \\
\hline \multicolumn{3}{|c|}{ lower $95 \%(\mu \mathrm{m})$ : } & 0.31 & $\mathrm{R}^{2}$ & 0.9876 \\
\hline \multirow{2}{*}{\multicolumn{3}{|c|}{ upper $95 \%(\mu \mathrm{m})$ : }} & 29.43 & lower $95 \%(\mu \mathrm{m})$ : & 0.40 \\
\hline & & & & upper $95 \%(\mu \mathrm{m})$ : & 24.30 \\
\hline
\end{tabular}

1. based on overall filter area of $1.59 \times 10^{9} \mu \mathrm{m}^{2}$

2. $\mathrm{GMD}=$ geometric mean diameter, $\mathrm{GSD}=$ geometric standard deviation, $\mathrm{R}=$ linear correlation coefficient

Figure 3.44 Module IV, Sample 36 (TSFH02-03) count-based particle size distribution. 


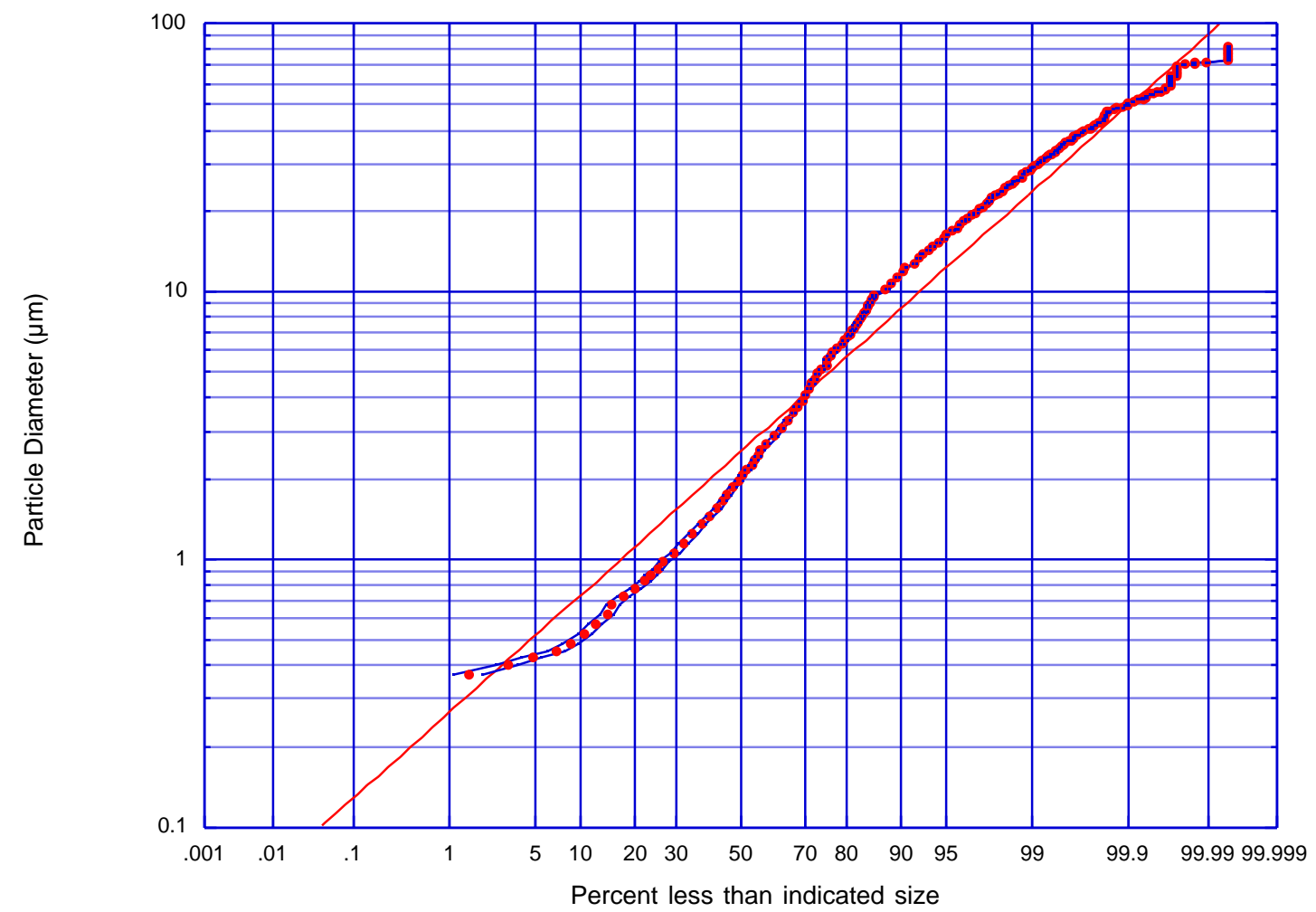

Analysis Summary Table:

\begin{tabular}{|c|c|c|c|c|c|}
\hline \multicolumn{2}{|c|}{$\begin{array}{c}\text { magnification, } \\
\text { number of images }\end{array}$} & \multicolumn{2}{|c|}{ area analyzed $^{1}(\%)$} & counted size range $(\mu \mathrm{m})$ & $\begin{array}{c}\text { total number of } \\
\text { particles (not scaled) }\end{array}$ \\
\hline $50 x$ & 6 & \multicolumn{2}{|r|}{0.894} & $d_{p}>9.65$ & 4301 \\
\hline $100 x$ & 6 & \multicolumn{2}{|r|}{0.224} & $5.13<d_{p}<9.65$ & 2871 \\
\hline $200 x$ & 6 & \multicolumn{2}{|r|}{0.0558} & $2.58<d_{p}<5.13$ & 1958 \\
\hline $500 x$ & 6 & \multicolumn{2}{|r|}{0.00893} & $1.44<\mathrm{d}_{\mathrm{p}}<2.58$ & 1084 \\
\hline 1000x, & 6 & \multicolumn{2}{|r|}{0.00217} & $0.00<\mathrm{d}_{\mathrm{p}}<1.44$ & 570 \\
\hline \multicolumn{2}{|c|}{ Totals: } & \multicolumn{2}{|r|}{$1.18 \%$} & - & 10784 \\
\hline \multicolumn{4}{|c|}{ data moments ${ }^{2}:$} & \multicolumn{2}{|c|}{ log-normal fit moments ${ }^{2}$ : } \\
\hline \multicolumn{3}{|c|}{ GMD $(\mu \mathrm{m}):$} & 2.33 & GMD $(\mu \mathrm{m}):$ & 2.52 \\
\hline \multicolumn{2}{|c|}{ GSD: } & & 3.09 & GSD: & 2.62 \\
\hline \multicolumn{3}{|c|}{ lower $95 \%(\mu \mathrm{m})$ : } & 0.24 & $\mathrm{R}^{2}$ & 0.97812 \\
\hline \multirow{2}{*}{\multicolumn{3}{|c|}{ upper $95 \%(\mu \mathrm{m})$ : }} & 22.30 & lower $95 \%(\mu \mathrm{m})$ : & 0.37 \\
\hline & & & & upper $95 \%(\mu \mathrm{m})$ : & 17.34 \\
\hline
\end{tabular}

1. based on overall filter area of $1.59 \times 10^{9} \mu \mathrm{m}^{2}$

2. $\mathrm{GMD}=$ geometric mean diameter, $\mathrm{GSD}=$ geometric standard deviation, $\mathrm{R}=$ linear correlation coefficient

Figure 3.45 Module IV, Sample 37 (TSFH04-03) count-based particle size distribution. 


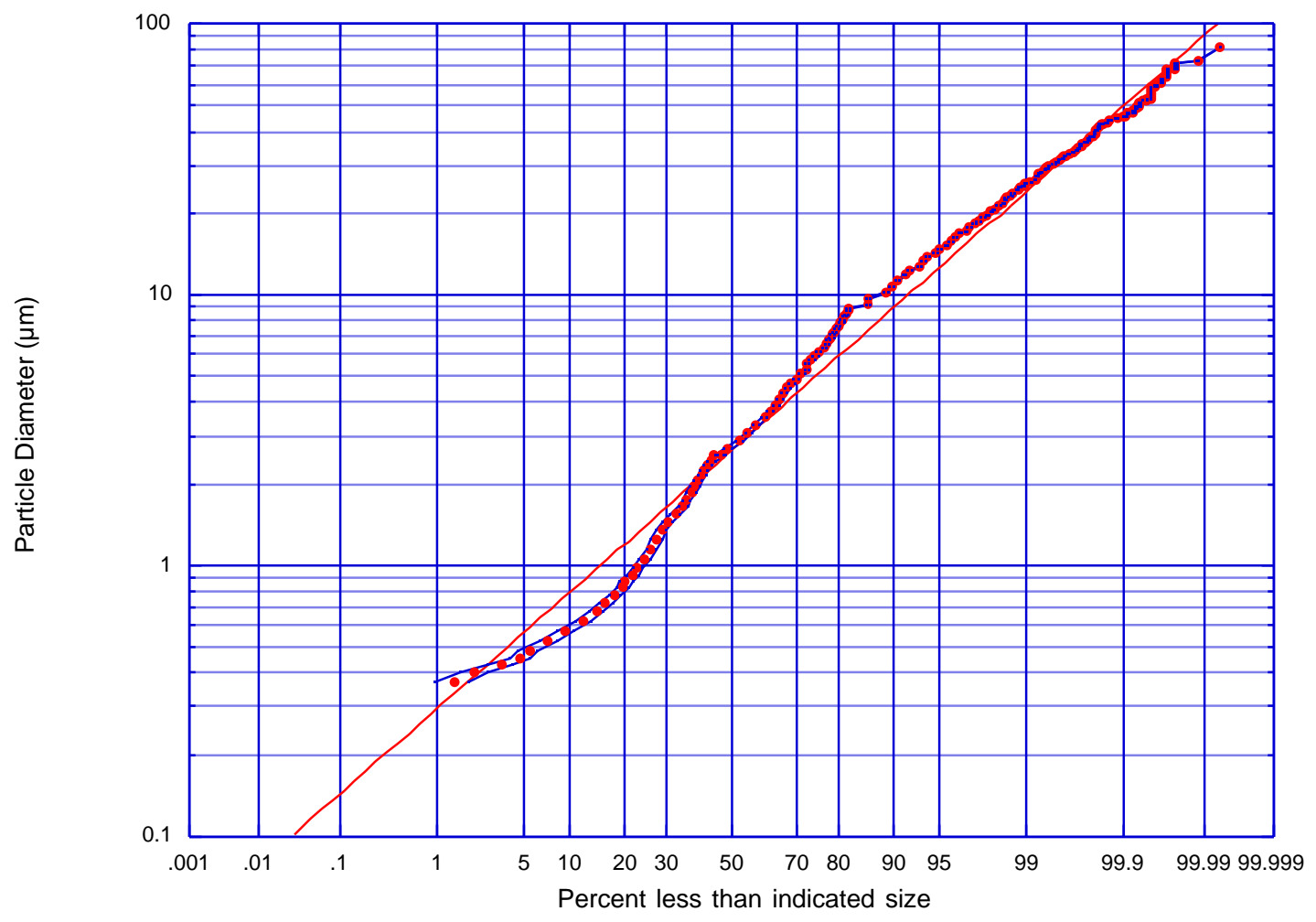

Analysis Summary Table:

\begin{tabular}{|c|c|c|c|c|c|}
\hline \multicolumn{2}{|c|}{$\begin{array}{c}\text { magnification, } \\
\text { number of images }\end{array}$} & \multicolumn{2}{|c|}{ area analyzed $^{1}(\%)$} & counted size range $(\mu \mathrm{m})$ & $\begin{array}{c}\text { total number of } \\
\text { particles (not scaled) }\end{array}$ \\
\hline $50 x$ & 6 & \multicolumn{2}{|r|}{0.894} & $d_{p}>8.99$ & 3814 \\
\hline $100 x$ & 6 & \multicolumn{2}{|r|}{0.224} & $4.85<\mathrm{d}_{\mathrm{p}}<8.99$ & 2530 \\
\hline $200 x$ & 6 & \multicolumn{2}{|r|}{0.0558} & $2.63<d_{p}<4.85$ & 2121 \\
\hline $500 x$ & 6 & \multicolumn{2}{|r|}{0.00893} & $1.49<\mathrm{d}_{\mathrm{p}}<2.63$ & 864 \\
\hline 1000x, & 6 & \multicolumn{2}{|r|}{0.00217} & $0.00<\mathrm{d}_{\mathrm{p}}<1.49$ & 386 \\
\hline \multicolumn{2}{|c|}{ Totals: } & \multicolumn{2}{|r|}{$1.18 \%$} & - & 9715 \\
\hline \multicolumn{4}{|c|}{ data moments ${ }^{2}:$} & \multicolumn{2}{|c|}{ log-normal fit moments ${ }^{2}$ : } \\
\hline \multicolumn{3}{|c|}{ GMD $(\mu \mathrm{m}):$} & 2.72 & GMD $(\mu \mathrm{m}):$ & 2.67 \\
\hline \multicolumn{2}{|c|}{ GSD: } & & 2.94 & GSD: & 2.56 \\
\hline \multicolumn{3}{|c|}{ lower $95 \%(\mu \mathrm{m})$ : } & 0.31 & $\mathrm{R}^{2}$ & 0.99074 \\
\hline \multirow{2}{*}{\multicolumn{3}{|c|}{ upper $95 \%(\mu \mathrm{m})$ : }} & 23.47 & lower $95 \%(\mu \mathrm{m})$ : & 0.40 \\
\hline & & & & upper $95 \%(\mu \mathrm{m})$ : & 17.76 \\
\hline
\end{tabular}

1. based on overall filter area of $1.59 \times 10^{9} \mu \mathrm{m}^{2}$

2. $\mathrm{GMD}=$ geometric mean diameter, $\mathrm{GSD}=$ geometric standard deviation, $\mathrm{R}=$ linear correlation coefficient

Figure 3.46 Module IV, Sample 38 (TSFH12-02) count-based particle size distribution. 


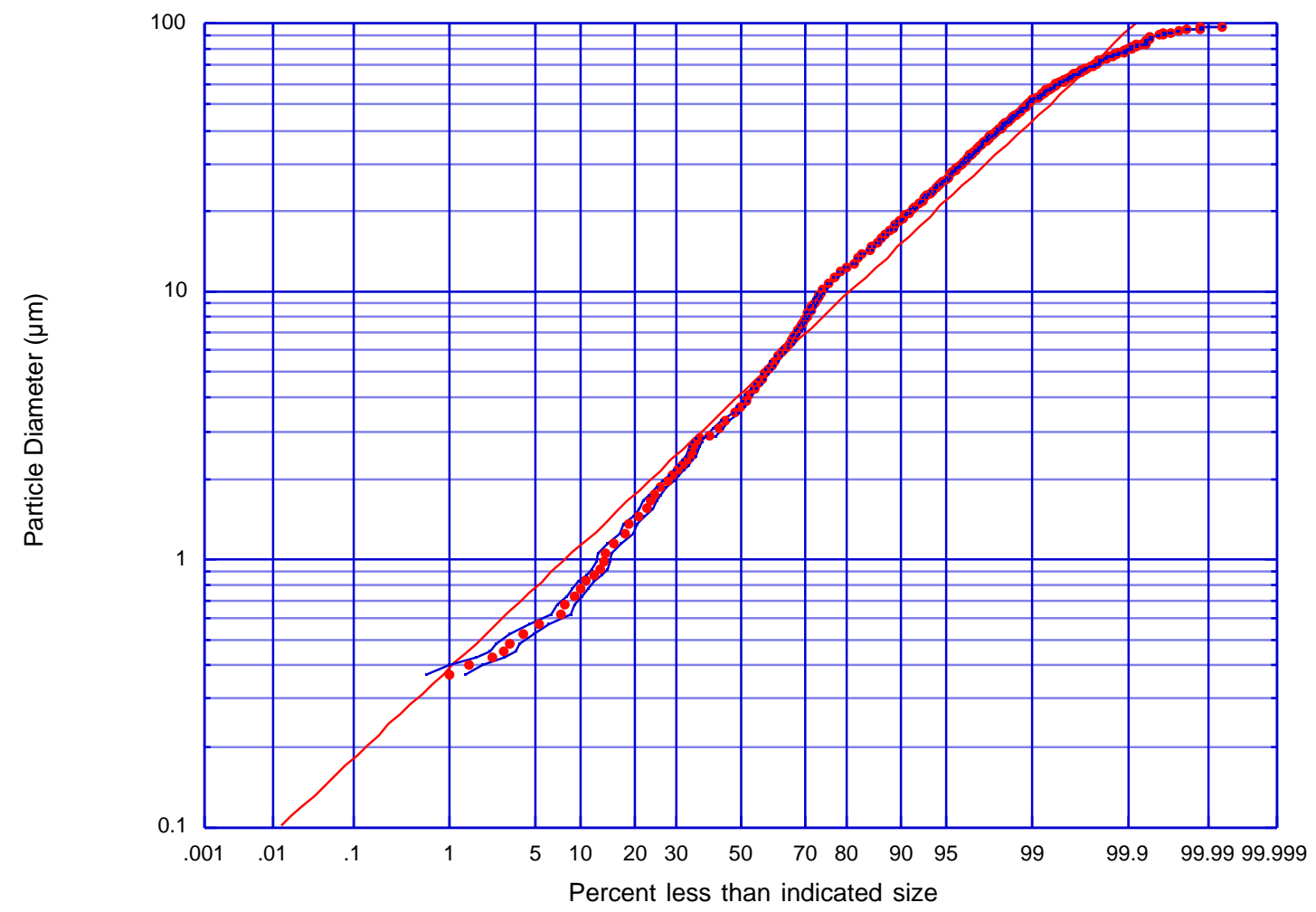

Analysis Summary Table:

\begin{tabular}{|c|c|c|c|c|c|}
\hline \multicolumn{2}{|c|}{$\begin{array}{c}\text { magnification, } \\
\text { number of images }\end{array}$} & \multicolumn{2}{|c|}{ area analyzed $^{1}(\%)$} & counted size range $(\mu \mathrm{m})$ & $\begin{array}{c}\text { total number of } \\
\text { particles (not scaled) }\end{array}$ \\
\hline $50 x$ & 6 & \multicolumn{2}{|r|}{0.894} & $d_{p}>10.72$ & 6644 \\
\hline $100 x$ & 6 & \multicolumn{2}{|r|}{0.224} & $5.74<\mathrm{d}_{\mathrm{p}}<10.72$ & 3098 \\
\hline $200 x$ & 6 & \multicolumn{2}{|r|}{0.0558} & $2.95<\mathrm{d}_{\mathrm{p}}<5.74$ & 2304 \\
\hline $500 x$ & 6 & \multicolumn{2}{|r|}{0.00893} & $1.75<d_{p}<2.95$ & 849 \\
\hline 1000x, & 6 & \multicolumn{2}{|r|}{0.00217} & $0.00<\mathrm{d}_{\mathrm{p}}<1.75$ & 324 \\
\hline \multicolumn{2}{|c|}{ Totals: } & \multicolumn{2}{|r|}{$1.18 \%$} & - & 13219 \\
\hline \multicolumn{4}{|c|}{ data moments ${ }^{2}:$} & \multicolumn{2}{|c|}{ log-normal fit moments ${ }^{2}$ : } \\
\hline \multicolumn{3}{|c|}{ GMD $(\mu \mathrm{m}):$} & 4.08 & GMD $(\mu \mathrm{m}):$ & 4.13 \\
\hline \multicolumn{2}{|c|}{ GSD: } & & 3.21 & GSD: & 2.76 \\
\hline \multicolumn{3}{|c|}{ lower $95 \%(\mu \mathrm{m})$ : } & 0.40 & $\mathrm{R}^{2}$ & 0.97927 \\
\hline \multirow{2}{*}{\multicolumn{3}{|c|}{ upper $95 \%(\mu \mathrm{m})$ : }} & 42.17 & lower $95 \%(\mu \mathrm{m})$ : & 0.54 \\
\hline & & & & upper $95 \%(\mu \mathrm{m})$ : & 31.38 \\
\hline
\end{tabular}

1. based on overall filter area of $1.59 \times 10^{9} \mu \mathrm{m}^{2}$

2. $\mathrm{GMD}=$ geometric mean diameter, $\mathrm{GSD}=$ geometric standard deviation, $\mathrm{R}=$ linear correlation coefficient

Figure 3.47 Module IV, Sample 39 (TSFH03-03) count-based particle size distribution. 


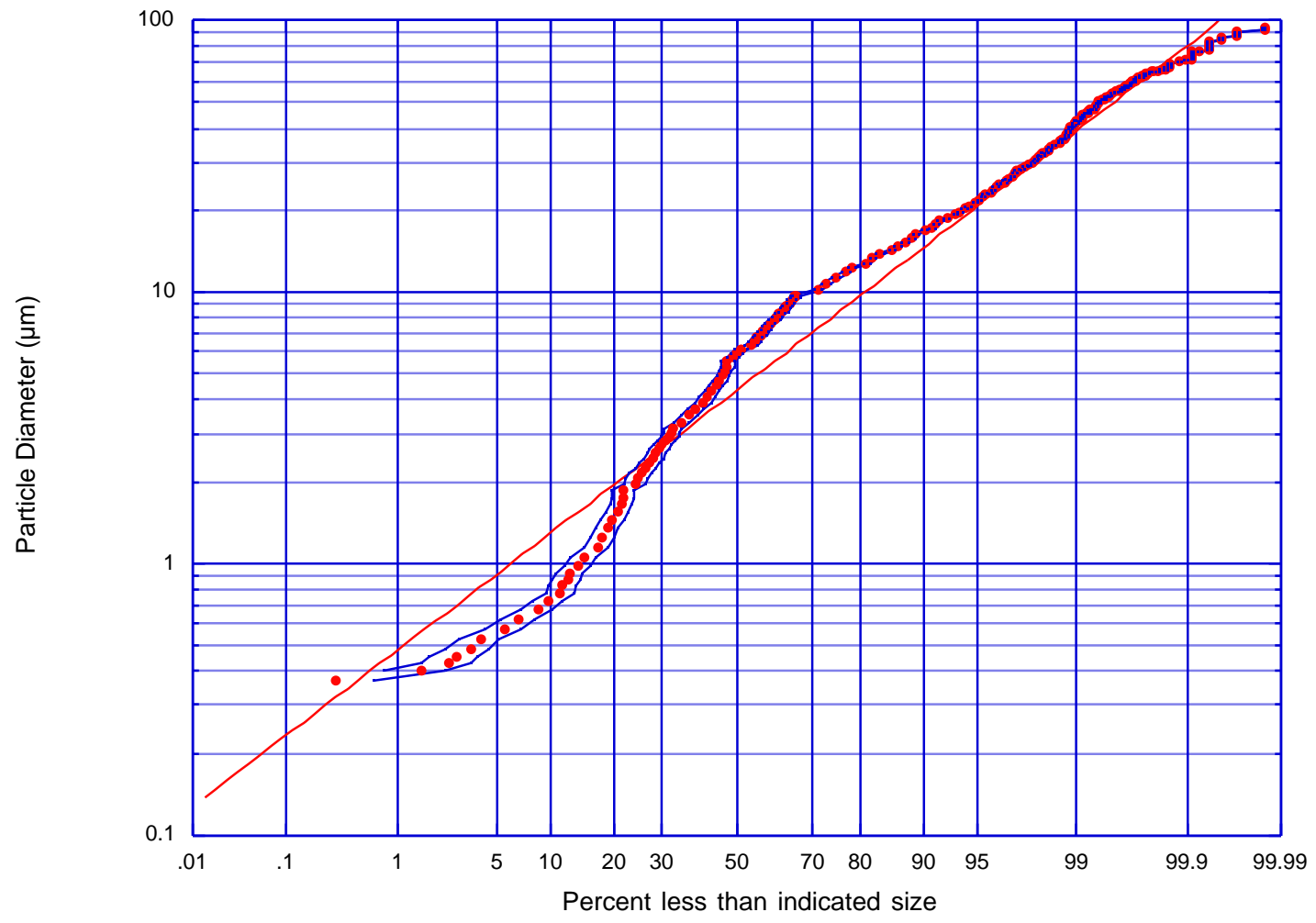

Analysis Summary Table:

\begin{tabular}{|c|c|c|c|c|c|}
\hline \multicolumn{2}{|c|}{$\begin{array}{c}\text { magnification, } \\
\text { number of images }\end{array}$} & \multicolumn{2}{|c|}{ area analyzed $^{1}(\%)$} & counted size range $(\mu \mathrm{m})$ & $\begin{array}{c}\text { total number of } \\
\text { particles (not scaled) }\end{array}$ \\
\hline $50 x$ & 6 & \multicolumn{2}{|r|}{0.894} & $d_{p}>9.81$ & 3333 \\
\hline $100 x$ & 6 & \multicolumn{2}{|r|}{0.224} & $5.56<d_{p}<9.81$ & 1558 \\
\hline $200 x$ & 6 & \multicolumn{2}{|r|}{0.0558} & $3.24<d_{p}<5.56$ & 822 \\
\hline $500 x$ & 6 & \multicolumn{2}{|r|}{0.00893} & $2.04<\mathrm{d}_{\mathrm{p}}<3.24$ & 255 \\
\hline 1000x, & 6 & \multicolumn{2}{|r|}{0.00217} & $0.00<\mathrm{d}_{\mathrm{p}}<2.04$ & 123 \\
\hline \multicolumn{2}{|c|}{ Totals: } & \multicolumn{2}{|r|}{$1.18 \%$} & - & 6091 \\
\hline \multicolumn{4}{|c|}{ data moments ${ }^{2}:$} & \multicolumn{2}{|c|}{ log-normal fit moments ${ }^{2}$ : } \\
\hline \multicolumn{3}{|c|}{ GMD $(\mu \mathrm{m}):$} & 4.71 & GMD $(\mu \mathrm{m}):$ & 4.34 \\
\hline \multicolumn{2}{|c|}{ GSD: } & & 3.15 & GSD: & 2.58 \\
\hline \multicolumn{3}{|c|}{ lower $95 \%(\mu \mathrm{m})$ : } & 0.47 & $\mathrm{R}^{2}$ & 0.98025 \\
\hline \multirow{2}{*}{\multicolumn{3}{|c|}{ upper $95 \%(\mu \mathrm{m})$ : }} & 46.80 & lower $95 \%(\mu \mathrm{m})$ : & 0.66 \\
\hline & & & & upper $95 \%(\mu \mathrm{m})$ : & 28.84 \\
\hline
\end{tabular}

1. based on overall filter area of $1.59 \times 10^{9} \mu \mathrm{m}^{2}$

2. $\mathrm{GMD}=$ geometric mean diameter, $\mathrm{GSD}=$ geometric standard deviation, $\mathrm{R}=$ linear correlation coefficient

Figure 3.48 Module IV, Sample 40 (TSFH17-02) count-based particle size distribution. 


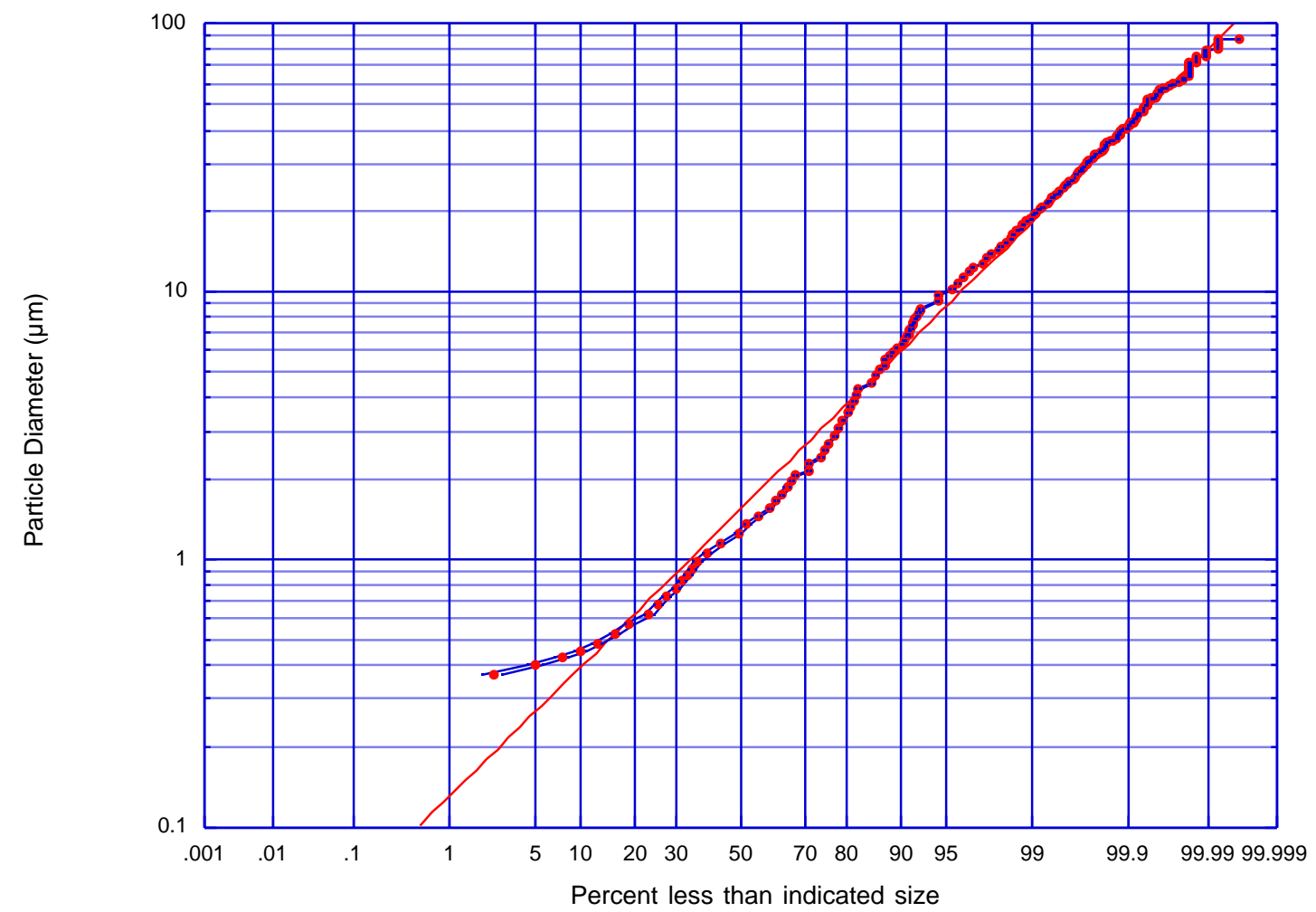

Analysis Summary Table:

\begin{tabular}{|c|c|c|c|c|c|}
\hline \multicolumn{2}{|c|}{$\begin{array}{c}\text { magnification, } \\
\text { number of images }\end{array}$} & \multicolumn{2}{|c|}{ area analyzed $^{1}(\%)$} & counted size range $(\mu \mathrm{m})$ & $\begin{array}{c}\text { total number of } \\
\text { particles (not scaled) }\end{array}$ \\
\hline $50 x$ & 6 & \multicolumn{2}{|r|}{0.894} & $d_{p}>8.60$ & 2906 \\
\hline $100 x$ & 6 & \multicolumn{2}{|r|}{0.224} & $4.41<\mathrm{d}_{\mathrm{p}}<8.60$ & 2261 \\
\hline $200 x$ & 6 & \multicolumn{2}{|r|}{0.0558} & $2.17<\mathrm{d}_{\mathrm{p}}<4.41$ & 1522 \\
\hline $500 x$ & 6 & \multicolumn{2}{|r|}{0.00893} & $1.10<d_{p}<2.17$ & 1836 \\
\hline 1000x, & 6 & \multicolumn{2}{|r|}{0.00217} & $0.00<\mathrm{d}_{\mathrm{p}}<1.10$ & 821 \\
\hline \multicolumn{2}{|c|}{ Totals: } & \multicolumn{2}{|r|}{$1.18 \%$} & - & 9346 \\
\hline \multicolumn{4}{|c|}{ data moments ${ }^{2}:$} & \multicolumn{2}{|c|}{ log-normal fit moments ${ }^{2}$ : } \\
\hline \multicolumn{3}{|c|}{ GMD $(\mu \mathrm{m}):$} & 1.54 & GMD $(\mu \mathrm{m}):$ & 1.54 \\
\hline \multicolumn{2}{|c|}{ GSD: } & & 2.67 & GSD: & 2.90 \\
\hline \multicolumn{3}{|c|}{ lower $95 \%(\mu \mathrm{m})$ : } & 0.22 & $\mathrm{R}^{2}$ & 0.99558 \\
\hline \multirow{2}{*}{\multicolumn{3}{|c|}{ upper $95 \%(\mu \mathrm{m})$ : }} & 10.97 & lower $95 \%(\mu \mathrm{m})$ : & 0.18 \\
\hline & & & & upper $95 \%(\mu \mathrm{m})$ : & 12.93 \\
\hline
\end{tabular}

1. based on overall filter area of $1.59 \times 10^{9} \mu \mathrm{m}^{2}$

2. $\mathrm{GMD}=$ geometric mean diameter, $\mathrm{GSD}=$ geometric standard deviation, $\mathrm{R}=$ linear correlation coefficient

Figure 3.49 Module IV, Sample 40A (TSFH19-02) count-based particle size distribution. 


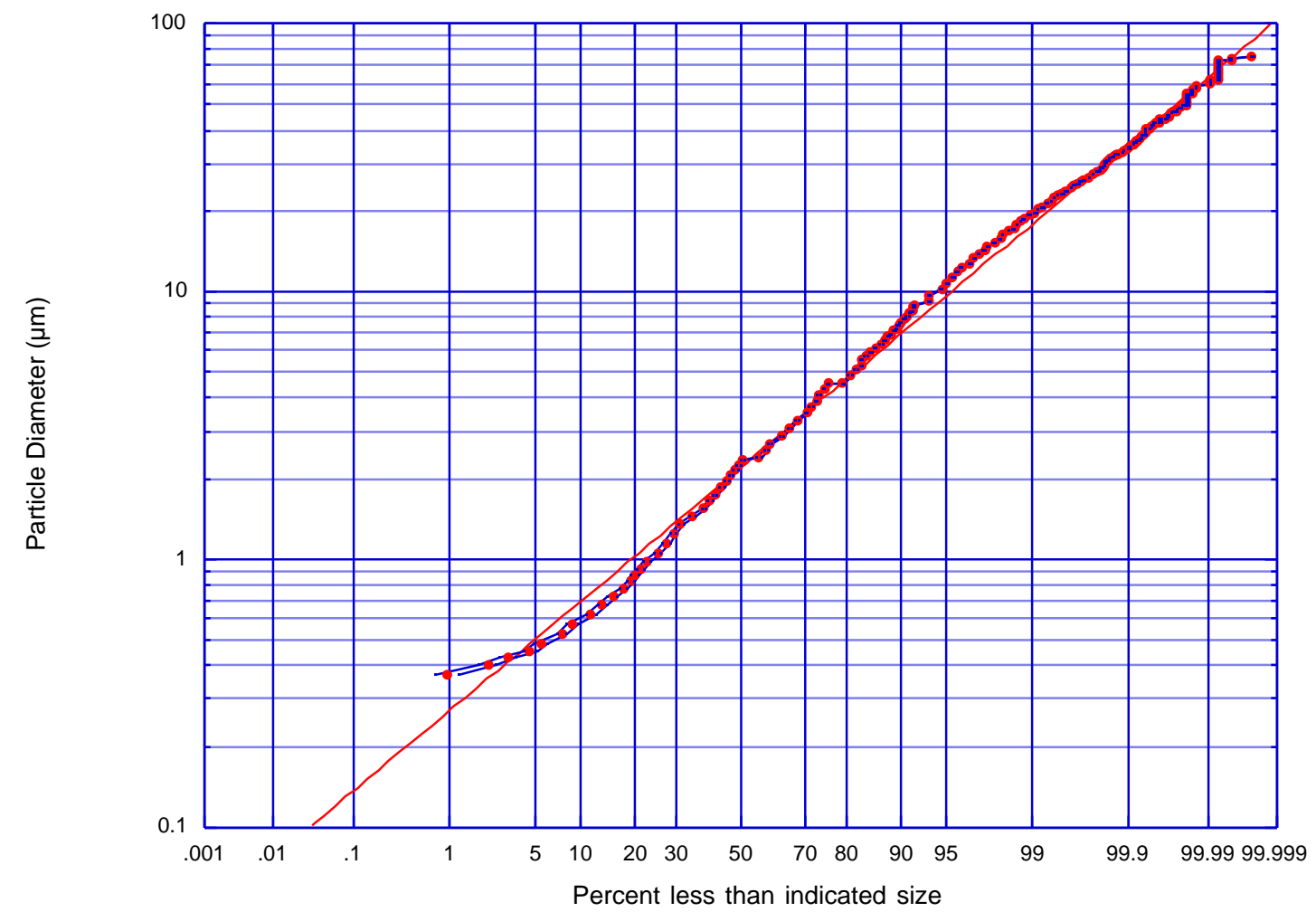

Analysis Summary Table:

\begin{tabular}{|c|c|c|c|c|c|}
\hline \multicolumn{2}{|c|}{$\begin{array}{c}\text { magnification, } \\
\text { number of images }\end{array}$} & \multicolumn{2}{|c|}{ area analyzed $^{1}(\%)$} & counted size range $(\mu \mathrm{m})$ & $\begin{array}{c}\text { total number of } \\
\text { particles (not scaled) }\end{array}$ \\
\hline $50 x$ & 6 & \multicolumn{2}{|r|}{0.894} & $d_{p}>8.99$ & 4503 \\
\hline $100 x$ & 6 & \multicolumn{2}{|r|}{0.224} & $4.73<\mathrm{d}_{\mathrm{p}}<8.99$ & 5400 \\
\hline $200 x$ & 6 & \multicolumn{2}{|r|}{0.0558} & $2.42<d_{p}<4.73$ & 4833 \\
\hline $500 x$ & 6 & \multicolumn{2}{|r|}{0.00893} & $1.33<\mathrm{d}_{\mathrm{p}}<2.42$ & 2485 \\
\hline 1000x, & 6 & \multicolumn{2}{|r|}{0.00217} & $0.00<\mathrm{d}_{\mathrm{p}}<1.33$ & 961 \\
\hline \multicolumn{2}{|c|}{ Totals: } & \multicolumn{2}{|r|}{$1.18 \%$} & - & 18182 \\
\hline \multicolumn{4}{|c|}{ data moments ${ }^{2}:$} & \multicolumn{2}{|c|}{ log-normal fit moments ${ }^{2}$ : } \\
\hline \multicolumn{3}{|c|}{ GMD $(\mu \mathrm{m}):$} & 2.22 & GMD $(\mu \mathrm{m}):$ & 2.20 \\
\hline \multicolumn{2}{|c|}{ GSD: } & & 2.57 & GSD: & 2.46 \\
\hline \multicolumn{3}{|c|}{ lower $95 \%(\mu \mathrm{m})$ : } & 0.34 & $\mathrm{R}^{2}$ & 0.99724 \\
\hline \multirow{2}{*}{\multicolumn{3}{|c|}{ upper $95 \%(\mu \mathrm{m})$ : }} & 14.58 & lower $95 \%(\mu \mathrm{m})$ : & 0.36 \\
\hline & & & & upper $95 \%(\mu \mathrm{m})$ : & 13.34 \\
\hline
\end{tabular}

1. based on overall filter area of $1.59 \times 10^{9} \mu \mathrm{m}^{2}$

2. $\mathrm{GMD}=$ geometric mean diameter, $\mathrm{GSD}=$ geometric standard deviation, $\mathrm{R}=$ linear correlation coefficient

Figure 3.50 Module IV, Sample 40B (TSFH15-02) count-based particle size distribution. 


\subsubsection{Module V Results}

Figure 3.51 through Figure 3.62 display details of particle size distributions for corresponding collection locations of Module V. Table 3.5 gives a summary of Module V results.

Table 3.5 Summary of dust collected from locations in Tore Supra Module V.

\begin{tabular}{|c|c|c|c|c|c|c|}
\hline \multirow{2}{*}{$\begin{array}{c}\text { Poloidal } \\
\text { Location }\end{array}$} & Sample & Filter ID & \multirow{2}{*}{$\begin{array}{c}\text { Sampled } \\
\text { Area }\left(\mathrm{cm}^{2}\right)\end{array}$} & $\begin{array}{c}\text { Collected } \\
\text { Mass }(\mathrm{mg})\end{array}$ & \multicolumn{2}{|c|}{$\begin{array}{c}\text { Count-based Size } \\
\text { Distribution Parameters }\end{array}$} \\
\hline \hline 1 & & & GMD $(\mu \mathrm{m})$ & GSD \\
\hline 2 & 41 & TSFH08-03 & 621.2 & 3.7 & 3.95 & 3.01 \\
\hline 4 & 42 & TSFH24-03 & 1,553 & 1.8 & 2.76 & 3.01 \\
\hline 3 & 44 & TSFH11-03 & 689.0 & 2.2 & 3.56 & 3.39 \\
\hline 6 & 45 & TSFH21-03 & 1,800 & 0.9 & 5.13 & 2.86 \\
\hline 5 & 46 & TSFH09-03 & 658.0 & 0.7 & 4.17 & 2.77 \\
\hline 7 & 47 & TSFH23-03 & 428.0 & 1.3 & 3.74 & 3.10 \\
\hline 8 & 48 & TSFH06-03 & 84.00 & 0.5 & 2.49 & 2.91 \\
\hline 10 & 49 & TSFH05-03 & 1,429 & 0.7 & 4.00 & 3.03 \\
\hline 9 & 50 & TSFH10-03 & 294.0 & 0.3 & 2.72 & 2.19 \\
\hline 11 & $50 \mathrm{~A}$ & TSFH22-03 & 160.0 & 21.2 & 1.85 & 2.78 \\
\hline 12 & $50 \mathrm{~B}$ & TSFH25-03 & 480.0 & 15.6 & 1.92 & 2.72 \\
\hline
\end{tabular}




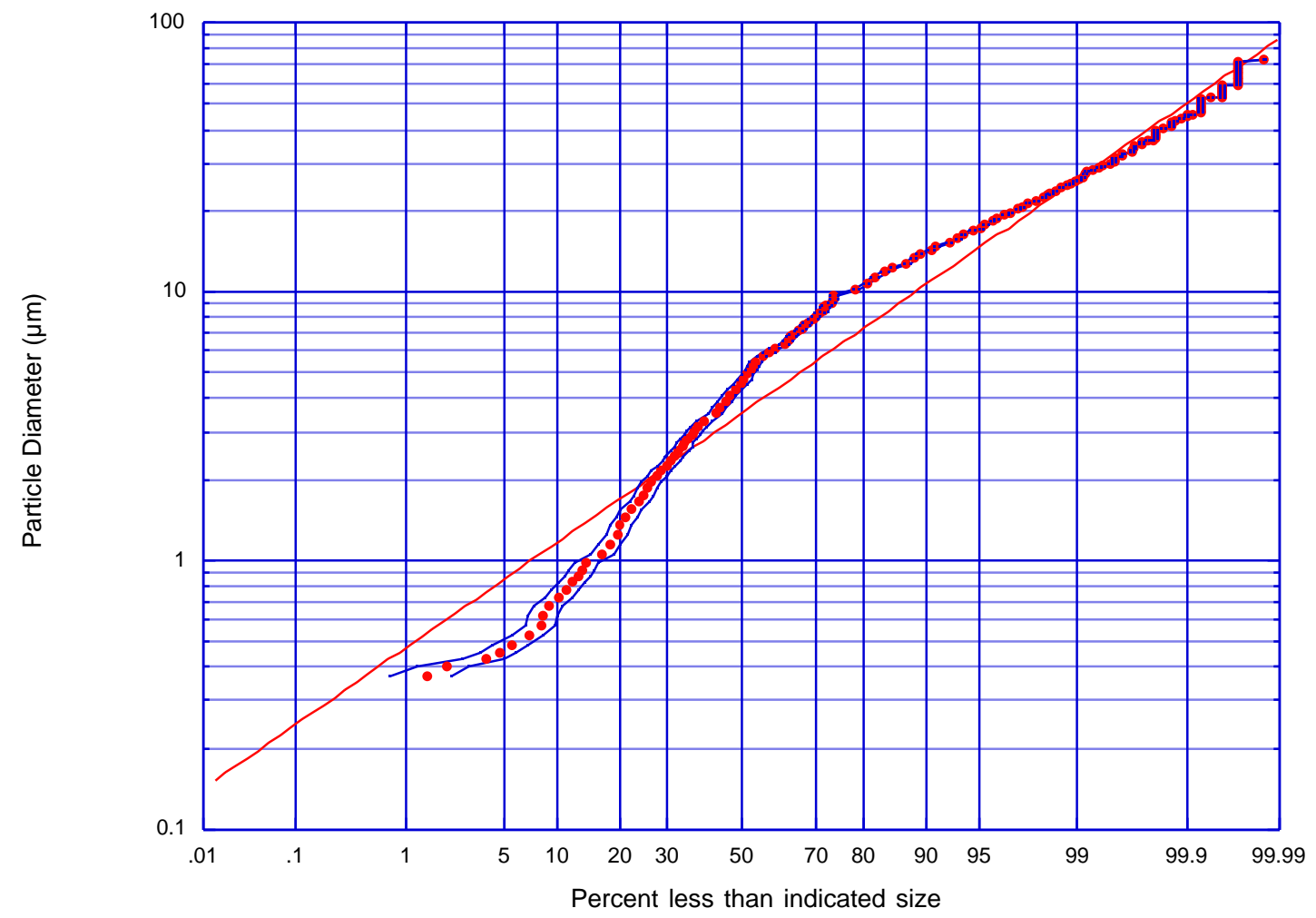

Analysis Summary Table:

\begin{tabular}{|c|c|c|c|c|c|}
\hline \multicolumn{2}{|c|}{$\begin{array}{c}\text { magnification, } \\
\text { number of images }\end{array}$} & \multicolumn{2}{|c|}{ area analyzed $^{1}(\%)$} & counted size range $(\mu \mathrm{m})$ & \multirow{2}{*}{$\begin{array}{c}\begin{array}{c}\text { total number of } \\
\text { particles (not scaled) }\end{array} \\
2846\end{array}$} \\
\hline $50 x$ & 6 & \multicolumn{2}{|r|}{0.894} & $d_{p}>9.36$ & \\
\hline $100 x$ & 6 & \multicolumn{2}{|r|}{0.224} & $5.62<d_{p}<9.36$ & 1726 \\
\hline $200 x$ & 6 & \multicolumn{2}{|r|}{0.0558} & $3.19<d_{p}<5.62$ & 875 \\
\hline $500 x$ & 6 & \multicolumn{2}{|r|}{0.00893} & $1.84<\mathrm{d}_{\mathrm{p}}<3.19$ & 290 \\
\hline 1000x, & 6 & \multicolumn{2}{|r|}{0.00217} & $0.00<\mathrm{d}_{\mathrm{p}}<1.84$ & 130 \\
\hline \multicolumn{2}{|c|}{ Totals: } & \multicolumn{2}{|r|}{$1.18 \%$} & - & 5867 \\
\hline \multicolumn{4}{|c|}{ data moments ${ }^{2}:$} & \multicolumn{2}{|c|}{ log-normal fit moments ${ }^{2}$ : } \\
\hline \multicolumn{3}{|c|}{ GMD $(\mu \mathrm{m}):$} & 3.95 & GMD $(\mu \mathrm{m}):$ & 3.52 \\
\hline \multicolumn{2}{|c|}{ GSD: } & & 3.01 & GSD: & 2.37 \\
\hline \multicolumn{3}{|c|}{ lower $95 \%(\mu \mathrm{m})$ : } & 0.44 & $\mathrm{R}^{2}:$ & 0.97473 \\
\hline \multicolumn{3}{|c|}{ upper $95 \%(\mu \mathrm{m})$ : } & 35.77 & lower $95 \%(\mu \mathrm{m})$ : & 0.63 \\
\hline & & & & upper $95 \%(\mu \mathrm{m})$ : & 19.80 \\
\hline
\end{tabular}

1. based on overall filter area of $1.59 \times 10^{9} \mu \mathrm{m}^{2}$

2. $\mathrm{GMD}=$ geometric mean diameter, $\mathrm{GSD}=$ geometric standard deviation, $\mathrm{R}=$ linear correlation coefficient

Figure 3.51 Module V, Sample 41 (TSFH08-03) count-based particle size distribution. 


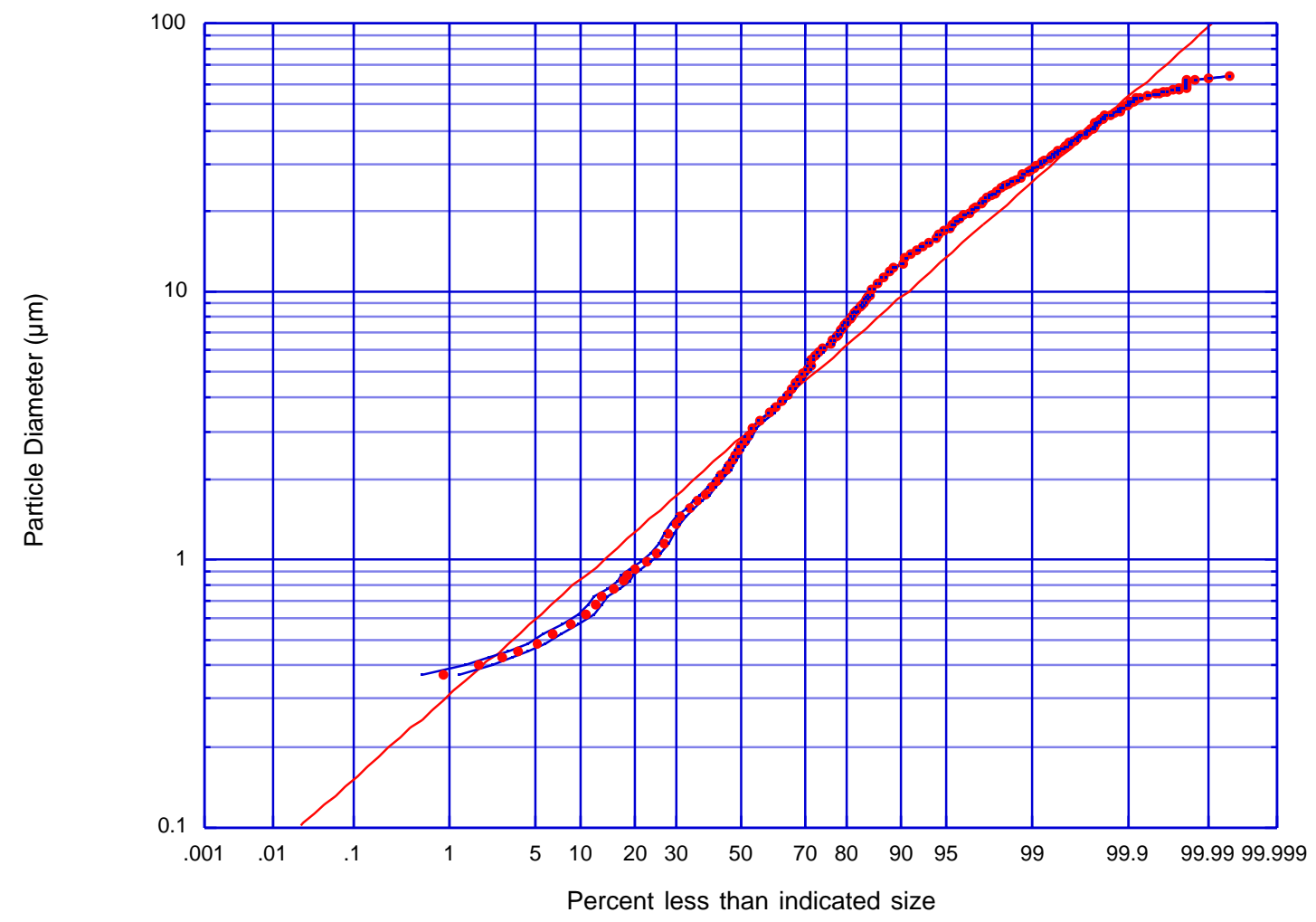

Analysis Summary Table:

\begin{tabular}{|c|c|c|c|c|c|}
\hline \multicolumn{2}{|c|}{$\begin{array}{c}\text { magnification, } \\
\text { number of images }\end{array}$} & \multicolumn{2}{|c|}{ area analyzed $^{1}(\%)$} & counted size range $(\mu \mathrm{m})$ & $\begin{array}{c}\text { total number of } \\
\text { particles (not scaled) }\end{array}$ \\
\hline $50 x$ & 6 & \multicolumn{2}{|r|}{0.894} & $d_{p}>9.75$ & 5316 \\
\hline $100 x$ & 6 & \multicolumn{2}{|r|}{0.224} & $5.40<d_{p}<9.75$ & 3807 \\
\hline $200 x$ & 6 & \multicolumn{2}{|r|}{0.0558} & $2.80<d_{p}<5.40$ & 3036 \\
\hline $500 x$ & 6 & \multicolumn{2}{|r|}{0.00893} & $1.54<\mathrm{d}_{\mathrm{p}}<2.80$ & 1313 \\
\hline 1000x, & 6 & \multicolumn{2}{|r|}{0.00217} & $0.00<\mathrm{d}_{\mathrm{p}}<1.54$ & 508 \\
\hline \multicolumn{2}{|c|}{ Totals: } & \multicolumn{2}{|r|}{$1.18 \%$} & - & 13980 \\
\hline \multicolumn{4}{|c|}{ data moments ${ }^{2}:$} & \multicolumn{2}{|c|}{ log-normal fit moments ${ }^{2}$ : } \\
\hline \multicolumn{3}{|c|}{ GMD $(\mu \mathrm{m}):$} & 2.76 & GMD $(\mu \mathrm{m}):$ & 2.83 \\
\hline \multicolumn{2}{|c|}{ GSD: } & & 3.01 & GSD: & 2.58 \\
\hline \multicolumn{3}{|c|}{ lower $95 \%(\mu \mathrm{m})$ : } & 0.30 & $\mathrm{R}^{2}$ & 0.98034 \\
\hline \multirow{2}{*}{\multicolumn{3}{|c|}{ upper $95 \%(\mu \mathrm{m})$ : }} & 25.10 & lower $95 \%(\mu \mathrm{m})$ : & 0.42 \\
\hline & & & & upper $95 \%(\mu \mathrm{m})$ : & 18.91 \\
\hline
\end{tabular}

1. based on overall filter area of $1.59 \times 10^{9} \mu \mathrm{m}^{2}$

2. $\mathrm{GMD}=$ geometric mean diameter, $\mathrm{GSD}=$ geometric standard deviation, $\mathrm{R}=$ linear correlation coefficient

Figure 3.52 Module V, Sample 42 (TSFH24-03) count-based particle size distribution. 


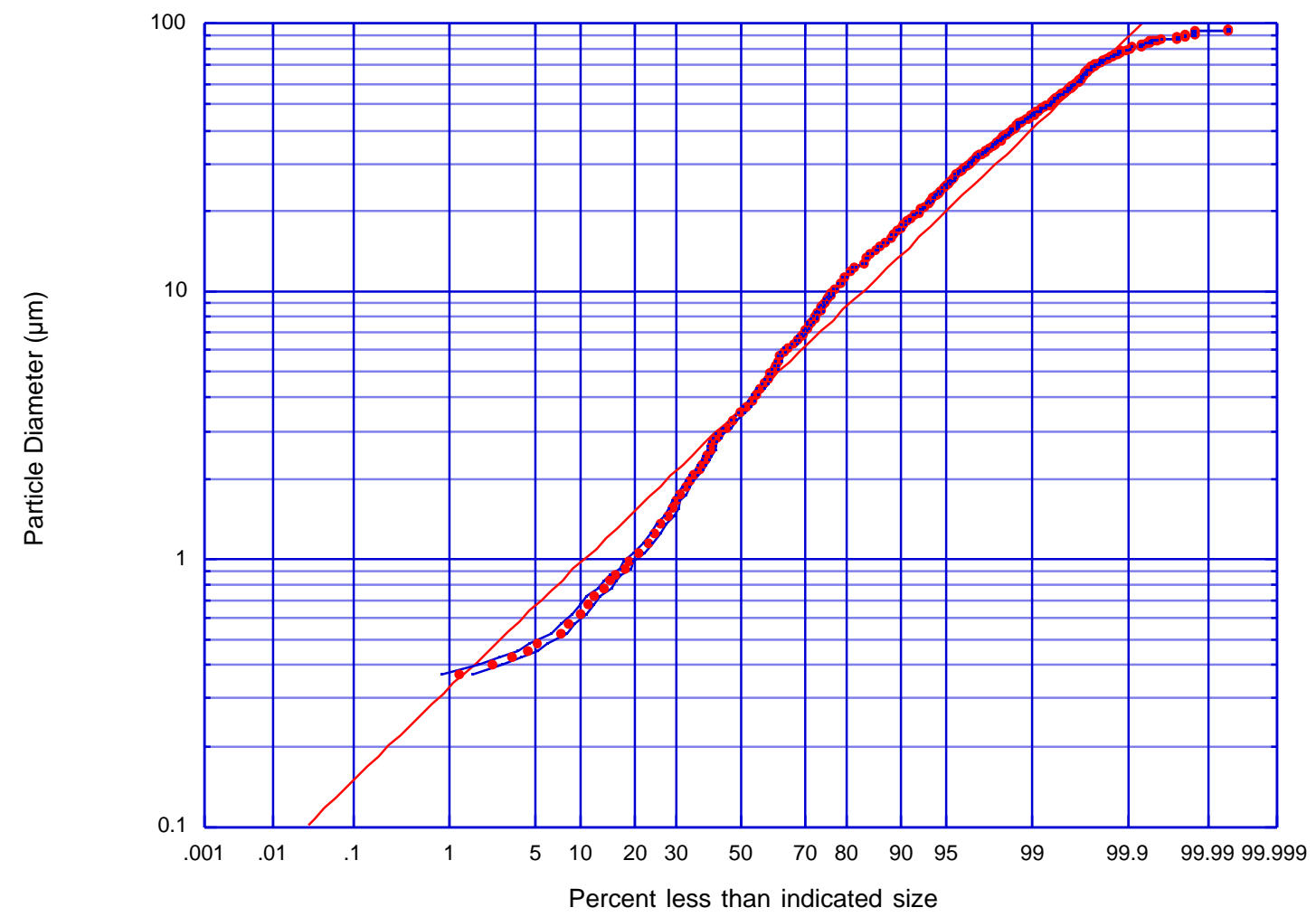

Analysis Summary Table:

\begin{tabular}{|c|c|c|c|c|c|}
\hline \multicolumn{2}{|c|}{$\begin{array}{c}\text { magnification, } \\
\text { number of images }\end{array}$} & \multicolumn{2}{|c|}{ area analyzed $^{1}(\%)$} & counted size range $(\mu \mathrm{m})$ & $\begin{array}{c}\text { total number of } \\
\text { particles (not scaled) }\end{array}$ \\
\hline $50 x$ & 6 & \multicolumn{2}{|r|}{0.894} & $d_{p}>10.78$ & 7063 \\
\hline $100 x$ & 6 & \multicolumn{2}{|r|}{0.224} & $5.79<\mathrm{d}_{\mathrm{p}}<10.78$ & 4262 \\
\hline $200 x$ & 6 & \multicolumn{2}{|r|}{0.0558} & $3.10<\mathrm{d}_{\mathrm{p}}<5.79$ & 2530 \\
\hline $500 x$ & 6 & \multicolumn{2}{|r|}{0.00893} & $1.81<\mathrm{d}_{\mathrm{p}}<3.10$ & 981 \\
\hline 1000x, & 6 & \multicolumn{2}{|r|}{0.00217} & $0.00<\mathrm{d}_{\mathrm{p}}<1.81$ & 497 \\
\hline \multicolumn{2}{|c|}{ Totals: } & \multicolumn{2}{|r|}{$1.18 \%$} & - & 15333 \\
\hline \multicolumn{4}{|c|}{ data moments ${ }^{2}:$} & \multicolumn{2}{|c|}{ log-normal fit moments ${ }^{2}$ : } \\
\hline \multicolumn{3}{|c|}{ GMD $(\mu \mathrm{m}):$} & 3.56 & GMD $(\mu \mathrm{m}):$ & 3.65 \\
\hline \multicolumn{2}{|c|}{ GSD: } & & 3.39 & GSD: & 2.82 \\
\hline \multicolumn{3}{|c|}{ lower $95 \%(\mu \mathrm{m})$ : } & 0.31 & $\mathrm{R}^{2}$ & 0.97621 \\
\hline \multirow{2}{*}{\multicolumn{3}{|c|}{ upper $95 \%(\mu \mathrm{m})$ : }} & 41.03 & lower $95 \%(\mu \mathrm{m})$ : & 0.46 \\
\hline & & & & upper $95 \%(\mu \mathrm{m})$ : & 28.94 \\
\hline
\end{tabular}

1. based on overall filter area of $1.59 \times 10^{9} \mu \mathrm{m}^{2}$

2. $\mathrm{GMD}=$ geometric mean diameter, $\mathrm{GSD}=$ geometric standard deviation, $\mathrm{R}=$ linear correlation coefficient

Figure 3.53 Module V, Sample 43 (TSFH11-03) count-based particle size distribution. 


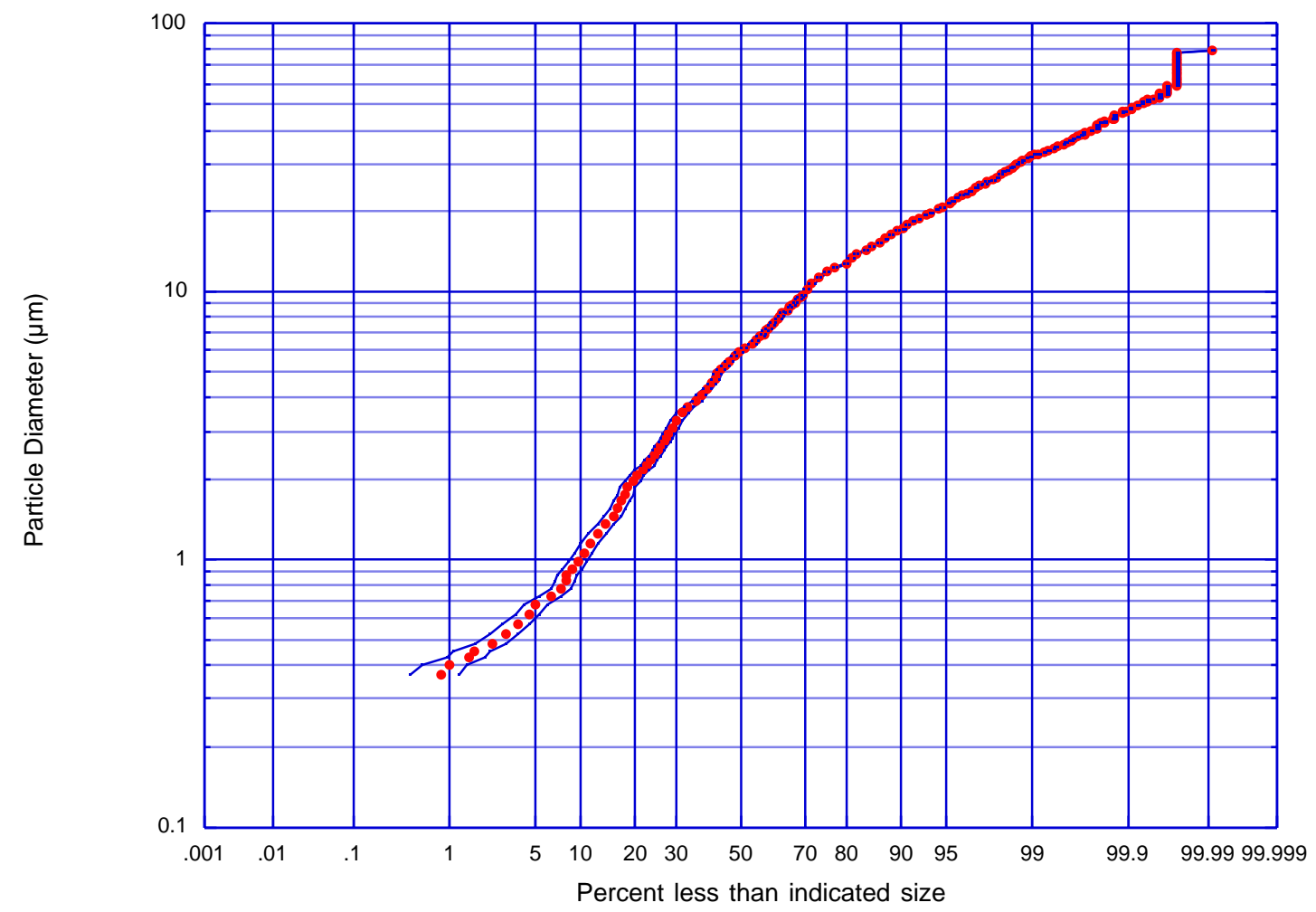

Analysis Summary Table:

\begin{tabular}{|c|c|c|c|c|c|}
\hline \multicolumn{2}{|c|}{$\begin{array}{c}\text { magnification, } \\
\text { number of images }\end{array}$} & \multicolumn{2}{|c|}{ area analyzed $^{1}(\%)$} & counted size range $(\mu \mathrm{m})$ & $\begin{array}{c}\text { total number of } \\
\text { particles (not scaled) }\end{array}$ \\
\hline $50 x$ & 6 & \multicolumn{2}{|r|}{0.894} & $d_{p}>9.77$ & 6335 \\
\hline $100 x$ & 6 & \multicolumn{2}{|r|}{0.224} & $5.61<d_{p}<9.77$ & 3489 \\
\hline $200 x$ & 6 & \multicolumn{2}{|r|}{0.0558} & $3.06<d_{p}<5.61$ & 1980 \\
\hline $500 x$ & 6 & \multicolumn{2}{|r|}{0.00893} & $1.86<\mathrm{d}_{\mathrm{p}}<3.06$ & 534 \\
\hline 1000x, & 6 & \multicolumn{2}{|r|}{0.00217} & $0.00<\mathrm{d}_{\mathrm{p}}<1.86$ & 190 \\
\hline \multicolumn{2}{|c|}{ Totals: } & \multicolumn{2}{|r|}{$1.18 \%$} & - & 12528 \\
\hline \multicolumn{4}{|c|}{ data moments ${ }^{2}$ : } & \multicolumn{2}{|c|}{ log-normal fit moments ${ }^{2}$ : } \\
\hline \multicolumn{3}{|c|}{ GMD $(\mu \mathrm{m}):$} & 5.13 & GMD $(\mu \mathrm{m}):$ & 4.40 \\
\hline \multicolumn{2}{|c|}{ GSD: } & & 2.86 & GSD: & 2.26 \\
\hline \multicolumn{3}{|c|}{ lower $95 \%(\mu \mathrm{m})$ : } & 0.63 & $\mathrm{R}^{2}$ & 0.97004 \\
\hline \multirow{2}{*}{\multicolumn{3}{|c|}{ upper $95 \%(\mu \mathrm{m})$ : }} & 41.95 & lower $95 \%(\mu \mathrm{m})$ : & 0.86 \\
\hline & & & & upper $95 \%(\mu \mathrm{m})$ : & 22.46 \\
\hline
\end{tabular}

1. based on overall filter area of $1.59 \times 10^{9} \mu \mathrm{m}^{2}$

2. $\mathrm{GMD}=$ geometric mean diameter, $\mathrm{GSD}=$ geometric standard deviation, $\mathrm{R}=$ linear correlation coefficient

Figure 3.54 Module V, Sample 44 (TSFH21-03) count-based particle size distribution. 


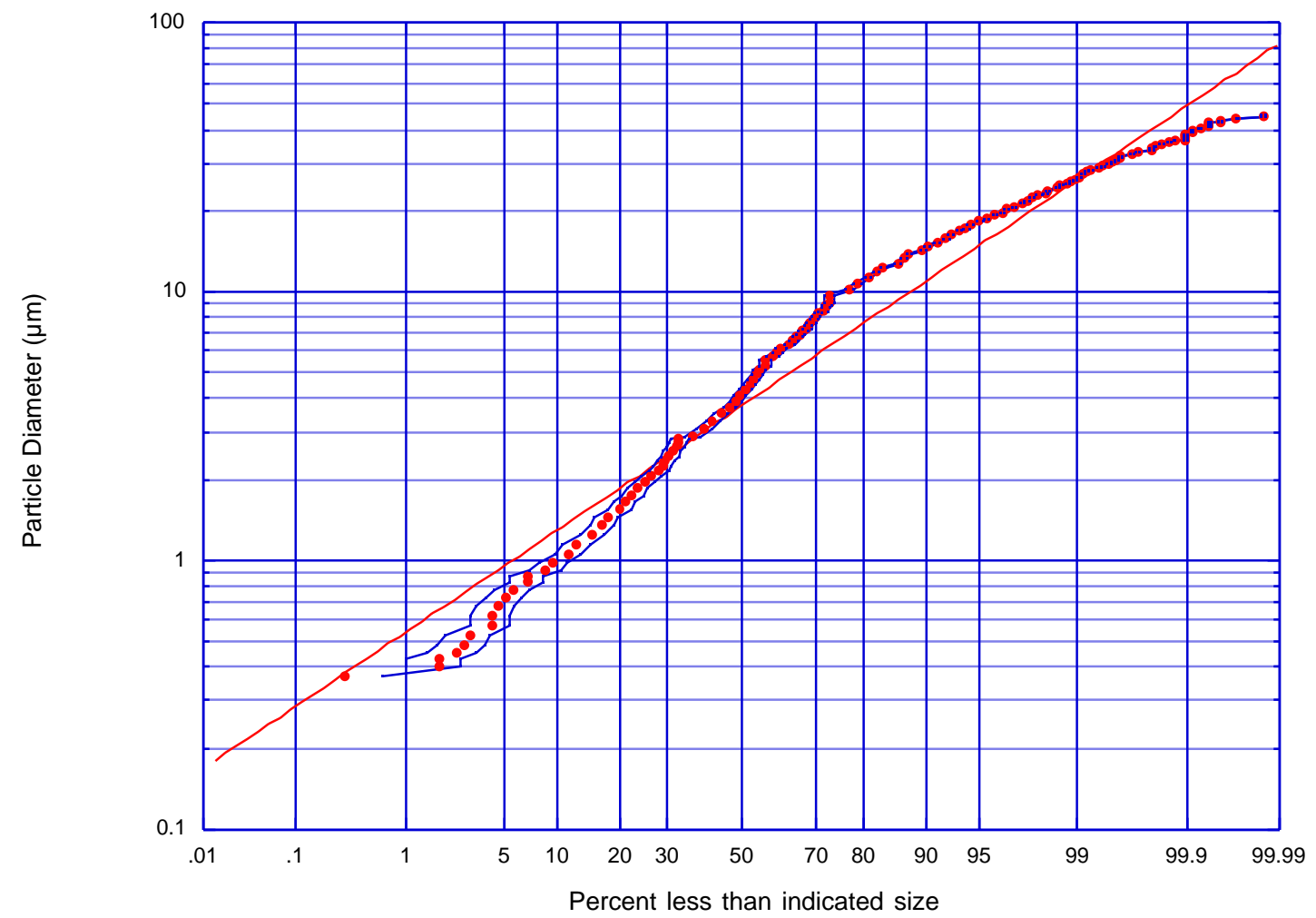

Analysis Summary Table:

\begin{tabular}{|c|c|c|c|c|c|}
\hline \multicolumn{2}{|c|}{$\begin{array}{c}\text { magnification, } \\
\text { number of images }\end{array}$} & \multicolumn{2}{|c|}{ area analyzed $^{1}(\%)$} & counted size range $(\mu \mathrm{m})$ & \multirow{2}{*}{$\begin{array}{c}\begin{array}{c}\text { total number of } \\
\text { particles (not scaled) }\end{array} \\
2819\end{array}$} \\
\hline $50 x$ & 6 & \multicolumn{2}{|r|}{0.894} & $d_{p}>9.22$ & \\
\hline $100 x$ & 6 & \multicolumn{2}{|r|}{0.224} & $5.21<d_{p}<9.22$ & 1538 \\
\hline $200 x$ & 6 & \multicolumn{2}{|r|}{0.0558} & $2.95<d_{p}<5.21$ & 901 \\
\hline $500 x$ & 6 & \multicolumn{2}{|r|}{0.00893} & $1.88<\mathrm{d}_{\mathrm{p}}<2.95$ & 272 \\
\hline 1000x, & 6 & \multicolumn{2}{|r|}{0.00217} & $0.00<\mathrm{d}_{\mathrm{p}}<1.88$ & 116 \\
\hline \multicolumn{2}{|c|}{ Totals: } & \multicolumn{2}{|r|}{$1.18 \%$} & - & 5646 \\
\hline \multicolumn{4}{|c|}{ data moments ${ }^{2}:$} & \multicolumn{2}{|c|}{ log-normal fit moments ${ }^{2}$ : } \\
\hline \multicolumn{3}{|c|}{ GMD $(\mu \mathrm{m}):$} & 4.17 & GMD $(\mu \mathrm{m}):$ & 3.76 \\
\hline \multicolumn{2}{|c|}{ GSD: } & & 2.77 & GSD: & 2.30 \\
\hline \multicolumn{3}{|c|}{ lower $95 \%(\mu \mathrm{m})$ : } & 0.54 & $\mathrm{R}^{2}:$ & 0.96822 \\
\hline \multicolumn{3}{|c|}{ upper $95 \%(\mu \mathrm{m})$ : } & 31.98 & lower $95 \%(\mu \mathrm{m})$ : & 0.71 \\
\hline & & & & upper $95 \%(\mu \mathrm{m})$ : & 19.95 \\
\hline
\end{tabular}

1. based on overall filter area of $1.59 \times 10^{9} \mu \mathrm{m}^{2}$

2. $\mathrm{GMD}=$ geometric mean diameter, $\mathrm{GSD}=$ geometric standard deviation, $\mathrm{R}=$ linear correlation coefficient

Figure 3.55 Module V, Sample 45 (TSFH09-03) count-based particle size distribution. 


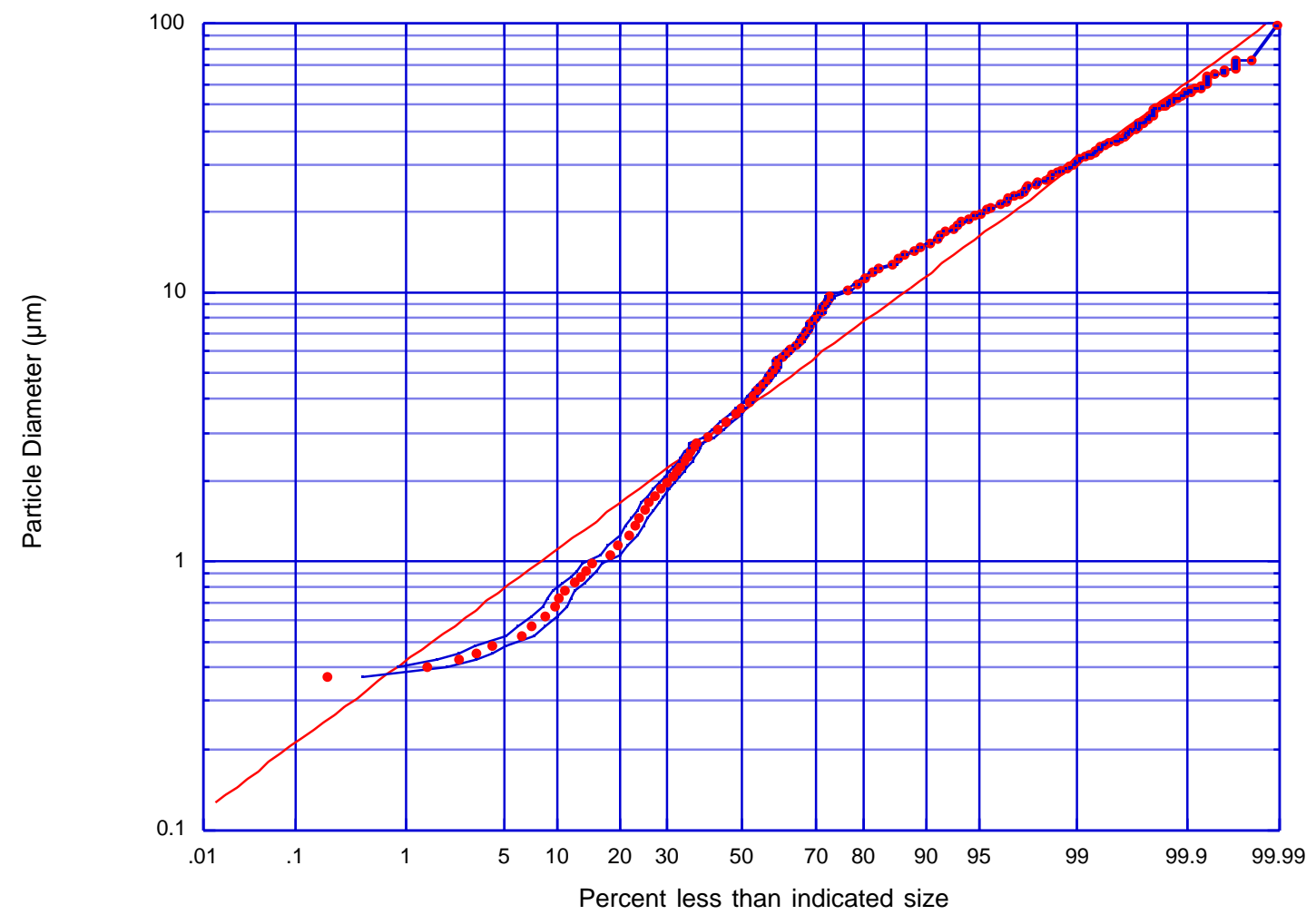

Analysis Summary Table:

\begin{tabular}{|c|c|c|c|c|c|}
\hline \multicolumn{2}{|c|}{$\begin{array}{c}\text { magnification, } \\
\text { number of images }\end{array}$} & \multicolumn{2}{|c|}{ area analyzed $^{1}(\%)$} & counted size range $(\mu \mathrm{m})$ & $\begin{array}{c}\text { total number of } \\
\text { particles (not scaled) }\end{array}$ \\
\hline $50 x$ & 6 & \multicolumn{2}{|r|}{0.894} & $d_{p}>9.70$ & 3848 \\
\hline $100 x$ & 6 & \multicolumn{2}{|r|}{0.224} & $5.39<d_{p}<9.70$ & 1761 \\
\hline $200 x$ & 6 & \multicolumn{2}{|r|}{0.0558} & $2.86<\mathrm{d}_{\mathrm{p}}<5.39$ & 1351 \\
\hline $500 x$ & 6 & \multicolumn{2}{|r|}{0.00893} & $1.69<\mathrm{d}_{\mathrm{p}}<2.86$ & 386 \\
\hline $1000 x$, & 6 & \multicolumn{2}{|r|}{0.00217} & $0.00<\mathrm{d}_{\mathrm{p}}<1.69$ & 192 \\
\hline \multicolumn{2}{|c|}{ Totals: } & \multicolumn{2}{|r|}{$1.18 \%$} & - & 7538 \\
\hline \multicolumn{4}{|c|}{ data moments ${ }^{2}:$} & \multicolumn{2}{|c|}{ log-normal fit moments ${ }^{2}$ : } \\
\hline \multicolumn{3}{|c|}{ GMD $(\mu \mathrm{m}):$} & 3.74 & GMD $(\mu \mathrm{m}):$ & 3.57 \\
\hline \multicolumn{2}{|c|}{ GSD: } & & 3.10 & GSD: & 2.50 \\
\hline \multicolumn{3}{|c|}{ lower $95 \%(\mu \mathrm{m})$ : } & 0.39 & $\mathrm{R}^{2}$ & 0.97976 \\
\hline \multirow{2}{*}{\multicolumn{3}{|c|}{ upper $95 \%(\mu \mathrm{m})$ : }} & 36.05 & lower $95 \%(\mu \mathrm{m})$ : & 0.57 \\
\hline & & & & upper $95 \%(\mu \mathrm{m})$ : & 22.36 \\
\hline
\end{tabular}

1. based on overall filter area of $1.59 \times 10^{9} \mu \mathrm{m}^{2}$

2. $\mathrm{GMD}=$ geometric mean diameter, $\mathrm{GSD}=$ geometric standard deviation, $\mathrm{R}=$ linear correlation coefficient

Figure 3.56 Module V, Sample 46 (TSFH07-03) count-based particle size distribution. 


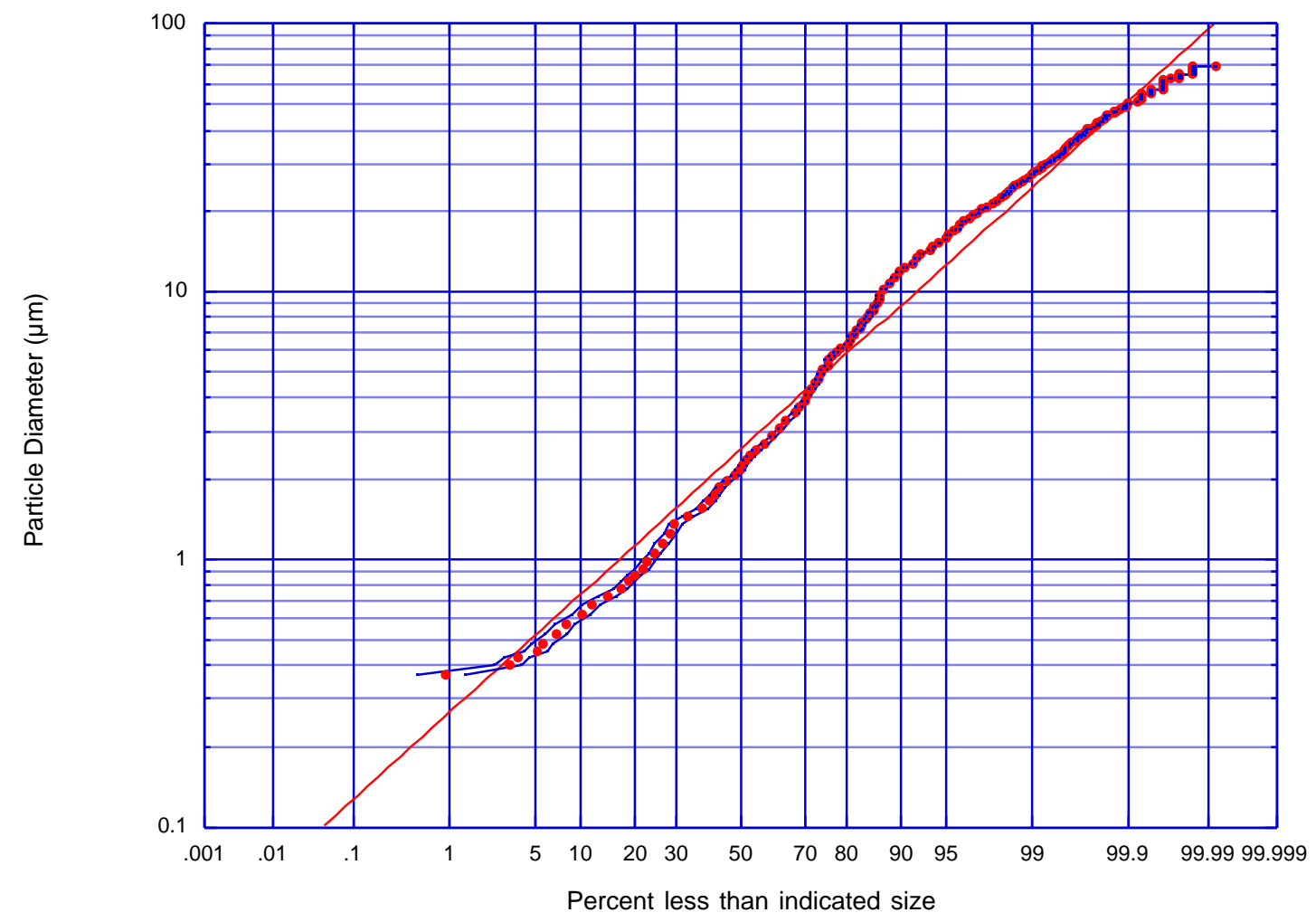

Analysis Summary Table:

\begin{tabular}{|c|c|c|c|c|c|}
\hline \multicolumn{2}{|c|}{$\begin{array}{c}\text { magnification, } \\
\text { number of images }\end{array}$} & \multicolumn{2}{|c|}{ area analyzed $^{1}(\%)$} & counted size range $(\mu \mathrm{m})$ & $\begin{array}{c}\text { total number of } \\
\text { particles (not scaled) }\end{array}$ \\
\hline $50 x$ & 6 & \multicolumn{2}{|r|}{0.894} & $d_{p}>9.52$ & 2943 \\
\hline $100 x$ & 6 & \multicolumn{2}{|r|}{0.224} & $5.08<d_{p}<9.52$ & 2068 \\
\hline $200 x$ & 6 & \multicolumn{2}{|r|}{0.0558} & $2.58<d_{p}<5.08$ & 1527 \\
\hline $500 x$ & 6 & \multicolumn{2}{|r|}{0.00893} & $1.40<\mathrm{d}_{\mathrm{p}}<2.58$ & 915 \\
\hline 1000x, & 6 & \multicolumn{2}{|r|}{0.00217} & $0.00<\mathrm{d}_{\mathrm{p}}<1.40$ & 285 \\
\hline \multicolumn{2}{|c|}{ Totals: } & \multicolumn{2}{|r|}{$1.18 \%$} & - & 7738 \\
\hline \multicolumn{4}{|c|}{ data moments ${ }^{2}:$} & \multicolumn{2}{|c|}{ log-normal fit moments ${ }^{2}$ : } \\
\hline \multicolumn{3}{|c|}{ GMD $(\mu \mathrm{m}):$} & 2.49 & GMD $(\mu \mathrm{m}):$ & 2.56 \\
\hline \multicolumn{2}{|c|}{ GSD: } & & 2.91 & GSD: & 2.65 \\
\hline \multicolumn{3}{|c|}{ lower $95 \%(\mu \mathrm{m})$ : } & 0.29 & $\mathrm{R}^{2}$ & 0.98798 \\
\hline \multirow{2}{*}{\multicolumn{3}{|c|}{ upper $95 \%(\mu \mathrm{m})$ : }} & 21.02 & lower $95 \%(\mu \mathrm{m})$ : & 0.37 \\
\hline & & & & upper $95 \%(\mu \mathrm{m})$ : & 18.00 \\
\hline
\end{tabular}

1. based on overall filter area of $1.59 \times 10^{9} \mu \mathrm{m}^{2}$

2. $\mathrm{GMD}=$ geometric mean diameter, $\mathrm{GSD}=$ geometric standard deviation, $\mathrm{R}=$ linear correlation coefficient

Figure 3.57 Module V, Sample 47 (TSFH23-03) count-based particle size distribution. 


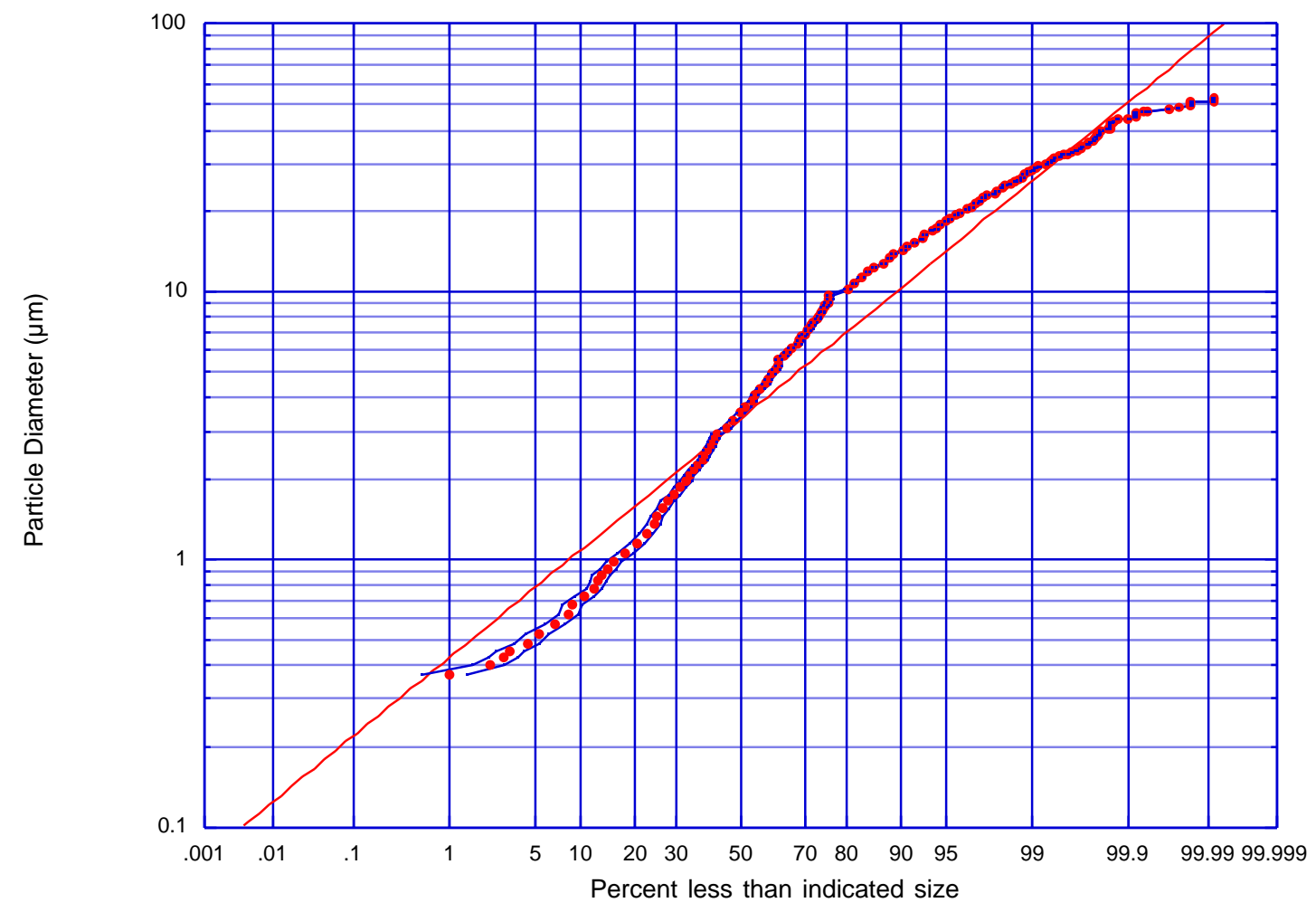

Analysis Summary Table:

\begin{tabular}{|c|c|c|c|c|c|}
\hline \multicolumn{2}{|c|}{$\begin{array}{c}\text { magnification, } \\
\text { number of images }\end{array}$} & \multicolumn{2}{|c|}{ area analyzed $^{1}(\%)$} & counted size range $(\mu \mathrm{m})$ & $\begin{array}{c}\text { total number of } \\
\text { particles (not scaled) }\end{array}$ \\
\hline $50 x$ & 6 & \multicolumn{2}{|r|}{0.894} & $d_{p}>9.48$ & 4491 \\
\hline $100 x$ & 6 & \multicolumn{2}{|r|}{0.224} & $5.49<\mathrm{d}_{\mathrm{p}}<9.48$ & 2393 \\
\hline $200 x$ & 6 & \multicolumn{2}{|r|}{0.0558} & $3.07<\mathrm{d}_{\mathrm{p}}<5.49$ & 1680 \\
\hline $500 x$ & 6 & \multicolumn{2}{|r|}{0.00893} & $1.85<\mathrm{d}_{\mathrm{p}}<3.07$ & 571 \\
\hline 1000x, & 6 & \multicolumn{2}{|r|}{0.00217} & $0.00<\mathrm{d}_{\mathrm{p}}<1.85$ & 300 \\
\hline \multicolumn{2}{|c|}{ Totals: } & \multicolumn{2}{|r|}{$1.18 \%$} & - & 9435 \\
\hline \multicolumn{4}{|c|}{ data moments ${ }^{2}:$} & \multicolumn{2}{|c|}{ log-normal fit moments ${ }^{2}$ : } \\
\hline \multicolumn{3}{|c|}{ GMD $(\mu \mathrm{m}):$} & 3.50 & GMD $(\mu \mathrm{m}):$ & 3.34 \\
\hline \multicolumn{2}{|c|}{ GSD: } & & 3.03 & GSD: & 2.42 \\
\hline \multicolumn{3}{|c|}{ lower $95 \%(\mu \mathrm{m})$ : } & 0.38 & $\mathrm{R}^{2}$ & 0.96461 \\
\hline \multirow{2}{*}{\multicolumn{3}{|c|}{ upper $95 \%(\mu \mathrm{m})$ : }} & 32.17 & lower $95 \%(\mu \mathrm{m})$ : & 0.57 \\
\hline & & & & upper $95 \%(\mu \mathrm{m})$ : & 19.52 \\
\hline
\end{tabular}

1. based on overall filter area of $1.59 \times 10^{9} \mu \mathrm{m}^{2}$

2. $\mathrm{GMD}=$ geometric mean diameter, $\mathrm{GSD}=$ geometric standard deviation, $\mathrm{R}=$ linear correlation coefficient

Figure 3.58 Module V, Sample 48 (TSFH06-03) count-based particle size distribution. 


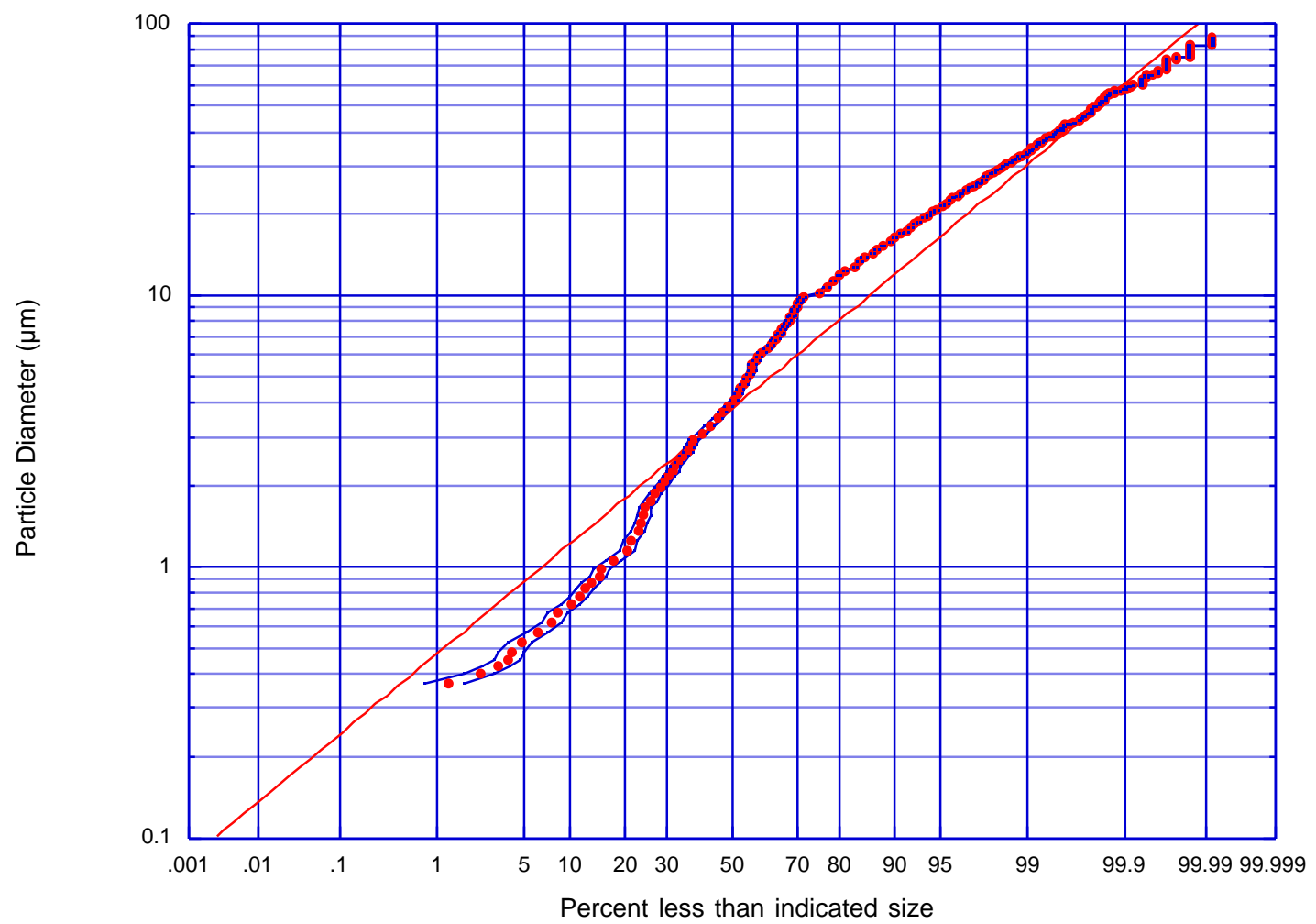

Analysis Summary Table:

\begin{tabular}{|c|c|c|c|c|c|}
\hline \multicolumn{2}{|c|}{$\begin{array}{c}\text { magnification, } \\
\text { number of images }\end{array}$} & \multicolumn{2}{|c|}{ area analyzed $^{1}(\%)$} & counted size range $(\mu \mathrm{m})$ & $\begin{array}{c}\text { total number of } \\
\text { particles (not scaled) }\end{array}$ \\
\hline $50 x$ & 6 & \multicolumn{2}{|r|}{0.894} & $\mathrm{~d}_{\mathrm{p}}>10.00$ & 5160 \\
\hline $100 x$ & 6 & \multicolumn{2}{|r|}{0.224} & $5.57<\mathrm{d}_{\mathrm{p}}<10.00$ & 2518 \\
\hline $200 x$ & 6 & \multicolumn{2}{|r|}{0.0558} & $3.03<\mathrm{d}_{\mathrm{p}}<5.57$ & 1614 \\
\hline $500 x$ & 6 & \multicolumn{2}{|r|}{0.00893} & $1.78<\mathrm{d}_{\mathrm{p}}<3.03$ & 594 \\
\hline 1000x, & 6 & \multicolumn{2}{|r|}{0.00217} & $0.00<\mathrm{d}_{\mathrm{p}}<1.78$ & 245 \\
\hline \multicolumn{2}{|c|}{ Totals: } & \multicolumn{2}{|r|}{$1.18 \%$} & - & 10131 \\
\hline \multicolumn{4}{|c|}{ data moments ${ }^{2}$ : } & \multicolumn{2}{|c|}{ log-normal fit moments ${ }^{2}$ : } \\
\hline \multicolumn{3}{|c|}{ GMD $(\mu \mathrm{m}):$} & 4.00 & GMD $(\mu \mathrm{m}):$ & 3.81 \\
\hline \multicolumn{2}{|c|}{ GSD: } & & 3.19 & GSD: & 2.45 \\
\hline \multicolumn{3}{|c|}{ lower $95 \%(\mu \mathrm{m})$ : } & 0.39 & $\mathrm{R}^{2}$ & 0.97381 \\
\hline \multirow{2}{*}{\multicolumn{3}{|c|}{ upper $95 \%(\mu \mathrm{m})$ : }} & 40.53 & lower $95 \%(\mu \mathrm{m})$ : & 0.64 \\
\hline & & & & upper $95 \%(\mu \mathrm{m})$ : & 22.80 \\
\hline
\end{tabular}

1. based on overall filter area of $1.59 \times 10^{9} \mu \mathrm{m}^{2}$

2. $\mathrm{GMD}=$ geometric mean diameter, $\mathrm{GSD}=$ geometric standard deviation, $\mathrm{R}=$ linear correlation coefficient

Figure 3.59 Module V, Sample 49 (TSFH05-03) count-based particle size distribution. 


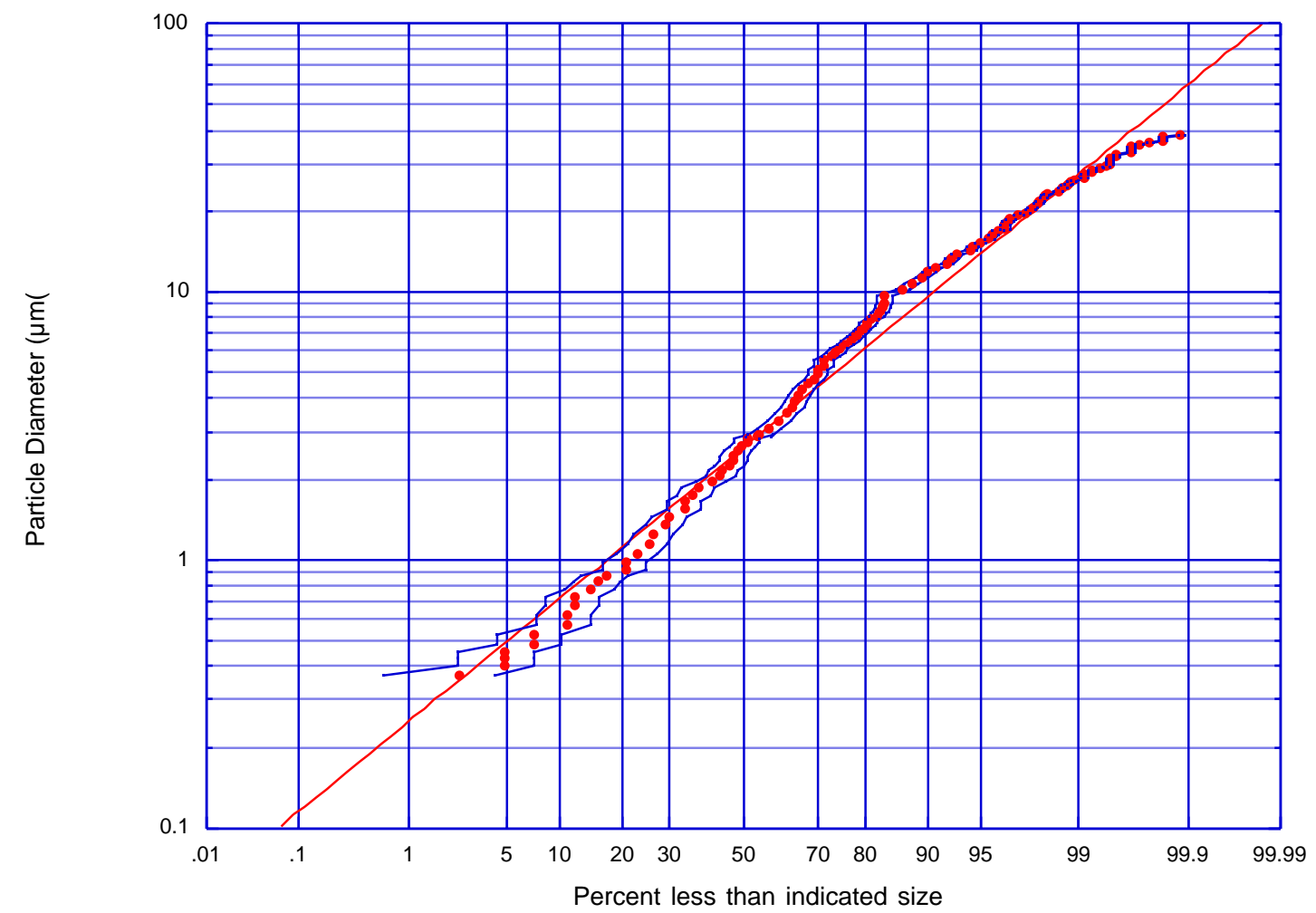

Analysis Summary Table:

\begin{tabular}{|c|c|c|c|c|c|}
\hline \multicolumn{2}{|c|}{$\begin{array}{c}\text { magnification, } \\
\text { number of images }\end{array}$} & \multicolumn{2}{|c|}{ area analyzed $^{1}(\%)$} & counted size range $(\mu \mathrm{m})$ & $\begin{array}{c}\text { total number of } \\
\text { particles (not scaled) }\end{array}$ \\
\hline $50 x$ & 6 & \multicolumn{2}{|r|}{0.894} & $d_{p}>9.24$ & 434 \\
\hline $100 x$ & 6 & \multicolumn{2}{|r|}{0.224} & $5.30<d_{p}<9.24$ & 307 \\
\hline $200 x$ & 6 & \multicolumn{2}{|r|}{0.0558} & $2.98<d_{p}<5.30$ & 223 \\
\hline $500 x$ & 6 & \multicolumn{2}{|r|}{0.00893} & $1.71<\mathrm{d}_{\mathrm{p}}<2.98$ & 90 \\
\hline 1000x, & 6 & \multicolumn{2}{|r|}{0.00217} & $0.00<\mathrm{d}_{\mathrm{p}}<1.71$ & 42 \\
\hline \multicolumn{2}{|c|}{ Totals: } & \multicolumn{2}{|r|}{$1.18 \%$} & - & 1096 \\
\hline \multicolumn{4}{|c|}{ data moments ${ }^{2}:$} & \multicolumn{2}{|c|}{ log-normal fit moments ${ }^{2}$ : } \\
\hline \multicolumn{3}{|c|}{ GMD $(\mu \mathrm{m}):$} & 2.72 & GMD $(\mu \mathrm{m}):$ & 2.62 \\
\hline \multicolumn{2}{|c|}{ GSD: } & & 2.96 & GSD: & 2.75 \\
\hline \multicolumn{3}{|c|}{ lower $95 \%(\mu \mathrm{m})$ : } & 0.31 & $\mathrm{R}^{2}$ & 0.98763 \\
\hline \multirow{2}{*}{\multicolumn{3}{|c|}{ upper $95 \%(\mu \mathrm{m})$ : }} & 23.90 & lower $95 \%(\mu \mathrm{m})$ : & 0.35 \\
\hline & & & & upper $95 \%(\mu \mathrm{m})$ : & 19.85 \\
\hline
\end{tabular}

1. based on overall filter area of $1.59 \times 10^{9} \mu \mathrm{m}^{2}$

2. $\mathrm{GMD}=$ geometric mean diameter, $\mathrm{GSD}=$ geometric standard deviation, $\mathrm{R}=$ linear correlation coefficient

Figure 3.60 Module V, Sample 50 (TSFH10-03) count-based particle size distribution. 


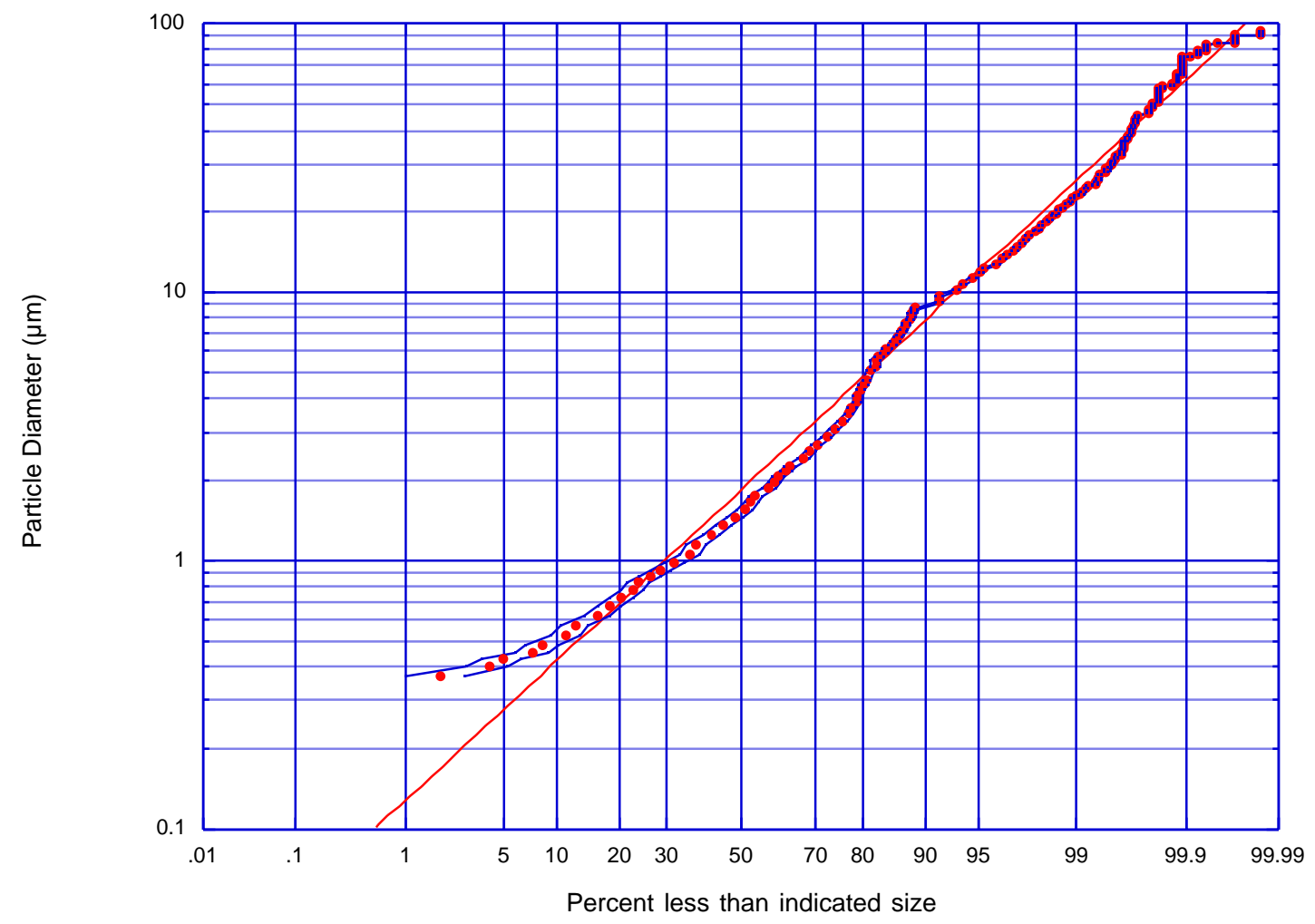

Analysis Summary Table:

\begin{tabular}{|c|c|c|c|c|c|}
\hline \multicolumn{2}{|c|}{$\begin{array}{c}\text { magnification, } \\
\text { number of images }\end{array}$} & \multicolumn{2}{|c|}{ area analyzed $^{1}(\%)$} & counted size range $(\mu \mathrm{m})$ & $\begin{array}{c}\text { total number of } \\
\text { particles (not scaled) }\end{array}$ \\
\hline $50 x$ & 6 & \multicolumn{2}{|r|}{0.894} & $d_{p}>8.83$ & 1010 \\
\hline $100 x$ & 6 & \multicolumn{2}{|r|}{0.224} & $4.78<\mathrm{d}_{\mathrm{p}}<8.83$ & 647 \\
\hline $200 x$ & 6 & \multicolumn{2}{|r|}{0.0558} & $2.34<d_{p}<4.78$ & 546 \\
\hline $500 x$ & 6 & \multicolumn{2}{|r|}{0.00893} & $1.27<\mathrm{d}_{\mathrm{p}}<2.34$ & 447 \\
\hline 1000x, & 6 & \multicolumn{2}{|r|}{0.00217} & $0.00<\mathrm{d}_{\mathrm{p}}<1.27$ & 191 \\
\hline \multicolumn{2}{|c|}{ Totals: } & \multicolumn{2}{|r|}{$1.18 \%$} & - & 2841 \\
\hline \multicolumn{4}{|c|}{ data moments ${ }^{2}$ : } & \multicolumn{2}{|c|}{ log-normal fit moments ${ }^{2}$ : } \\
\hline \multicolumn{3}{|c|}{ GMD $(\mu \mathrm{m}):$} & 1.85 & GMD $(\mu \mathrm{m}):$ & 1.83 \\
\hline \multicolumn{2}{|c|}{ GSD: } & & 2.78 & GSD: & 3.14 \\
\hline \multicolumn{3}{|c|}{ lower $95 \%(\mu \mathrm{m})$ : } & 0.24 & $\mathrm{R}^{2}$ & 0.99167 \\
\hline \multirow{2}{*}{\multicolumn{3}{|c|}{ upper $95 \%(\mu \mathrm{m})$ : }} & 14.31 & lower $95 \%(\mu \mathrm{m})$ : & 0.19 \\
\hline & & & & upper $95 \%(\mu \mathrm{m})$ : & 18.01 \\
\hline
\end{tabular}

1. based on overall filter area of $1.59 \times 10^{9} \mu \mathrm{m}^{2}$

2. $\mathrm{GMD}=$ geometric mean diameter, $\mathrm{GSD}=$ geometric standard deviation, $\mathrm{R}=$ linear correlation coefficient

Figure 3.61 Module V, Sample 50A (TSFH22-03) count-based particle size distribution. 


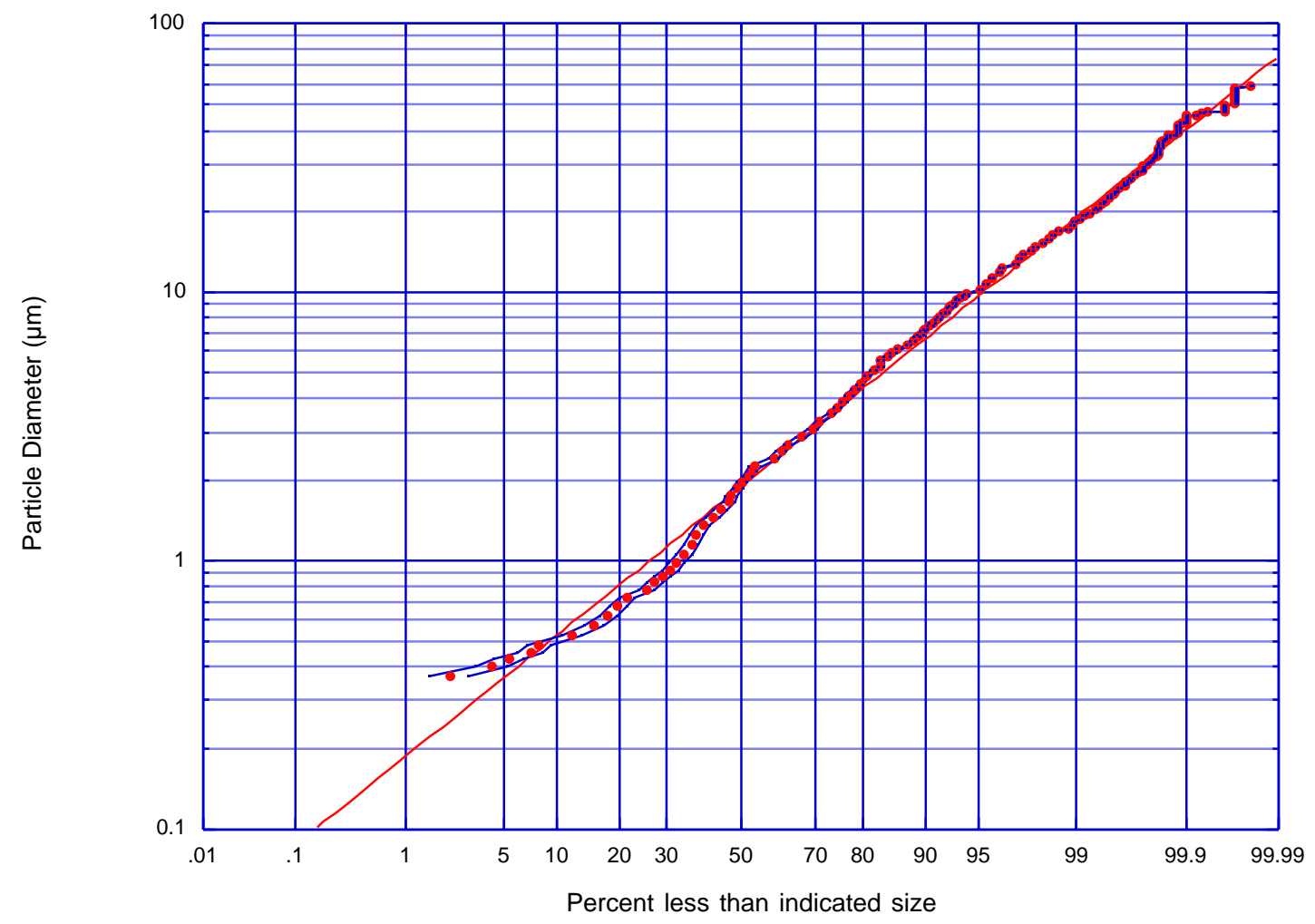

Analysis Summary Table:

\begin{tabular}{|c|c|c|c|c|c|}
\hline \multicolumn{2}{|c|}{$\begin{array}{c}\text { magnification, } \\
\text { number of images }\end{array}$} & \multicolumn{2}{|c|}{ area analyzed $^{1}(\%)$} & counted size range $(\mu \mathrm{m})$ & $\begin{array}{c}\text { total number of } \\
\text { particles (not scaled) }\end{array}$ \\
\hline $50 x$ & 6 & \multicolumn{2}{|r|}{0.894} & $d_{p}>8.94$ & 986 \\
\hline $100 x$ & 6 & \multicolumn{2}{|r|}{0.224} & $4.74<\mathrm{d}_{\mathrm{p}}<8.94$ & 1401 \\
\hline $200 x$ & 6 & \multicolumn{2}{|r|}{0.0558} & $2.34<d_{p}<4.74$ & 1126 \\
\hline $500 x$ & 6 & \multicolumn{2}{|r|}{0.00893} & $1.27<\mathrm{d}_{\mathrm{p}}<2.34$ & 529 \\
\hline 1000x, & 6 & \multicolumn{2}{|r|}{0.00217} & $0.00<\mathrm{d}_{\mathrm{p}}<1.27$ & 276 \\
\hline \multicolumn{2}{|c|}{ Totals: } & \multicolumn{2}{|r|}{$1.18 \%$} & - & 4318 \\
\hline \multicolumn{4}{|c|}{ data moments ${ }^{2}:$} & \multicolumn{2}{|c|}{ log-normal fit moments ${ }^{2}$ : } \\
\hline \multicolumn{3}{|c|}{ GMD $(\mu \mathrm{m}):$} & 1.92 & GMD $(\mu \mathrm{m}):$ & 1.88 \\
\hline \multicolumn{2}{|c|}{ GSD: } & & 2.72 & GSD: & 2.70 \\
\hline \multicolumn{3}{|c|}{ lower $95 \%(\mu \mathrm{m})$ : } & 0.26 & $\mathrm{R}^{2}$ & 0.99629 \\
\hline \multirow{2}{*}{\multicolumn{3}{|c|}{ upper $95 \%(\mu \mathrm{m})$ : }} & 14.26 & lower $95 \%(\mu \mathrm{m})$ : & 0.26 \\
\hline & & & & upper $95 \%(\mu \mathrm{m})$ : & 13.72 \\
\hline
\end{tabular}

1. based on overall filter area of $1.59 \times 10^{9} \mu \mathrm{m}^{2}$

2. $\mathrm{GMD}=$ geometric mean diameter, $\mathrm{GSD}=$ geometric standard deviation, $\mathrm{R}=$ linear correlation coefficient

Figure 3.62 Module V, Sample 50B (TSFH25-03) count-based particle size distribution. 


\subsubsection{Module VI Results}

Figure 3.63 through Figure 3.74 display details of particle size distributions for corresponding collection locations of Module VI. Table 3.6 gives a summary of Module VI results.

Table 3.6 Summary of dust collected from locations in Tore Supra Module VI.

\begin{tabular}{|c|c|c|c|c|c|c|}
\hline \multirow{2}{*}{$\begin{array}{c}\text { Poloidal } \\
\text { Location }\end{array}$} & Sample & Filter ID & $\begin{array}{c}\text { Sampled } \\
\text { Area }\left(\mathrm{cm}^{2}\right)\end{array}$ & $\begin{array}{c}\text { Collected } \\
\text { Mass }(\mathrm{mg})\end{array}$ & \multicolumn{2}{|c|}{$\begin{array}{c}\text { Count-based Size } \\
\text { Distribution Parameters }\end{array}$} \\
\hline \hline 1 & & & GMD $(\mu \mathrm{m})$ & GSD \\
\hline 2 & 51 & TSFH12-03 & 621.2 & 0.3 & 1.77 & 2.58 \\
\hline 4 & 52 & TSFH17-03 & 1,553 & $1.9^{*}$ & 2.72 & 2.99 \\
\hline 3 & 53 & TSFH13-03 & 689.0 & $1.0^{*}$ & 2.69 & 2.99 \\
\hline 6 & 54 & TSFH27-02 & 1,800 & 0.6 & 2.66 & 2.78 \\
\hline 5 & 56 & TSFH26-03 & 658.0 & 0.0 & 2.39 & 2.85 \\
\hline 7 & 57 & TSFH27-01 & 1,200 & 1.6 & 3.71 & 2.75 \\
\hline 8 & 58 & TSFH14-03 & 428.0 & 1.4 & 2.69 & 3.23 \\
\hline 10 & 59 & TSFH19-03 & 84.00 & 0.2 & 3.53 & 2.84 \\
\hline 9 & 60 & TSFH15-03 & 294.0 & $0.1^{*}$ & 2.52 & 3.00 \\
\hline 11 & $60 \mathrm{~A}$ & TSFH16-03 & 160.0 & $46.9^{*}$ & 1.54 & 2.03 \\
\hline 12 & $60 \mathrm{~B}$ & TSFH18-03 & 480.0 & $41.4^{*}$ & 2.25 & 3.02 \\
\hline
\end{tabular}

*- filter partially broken 


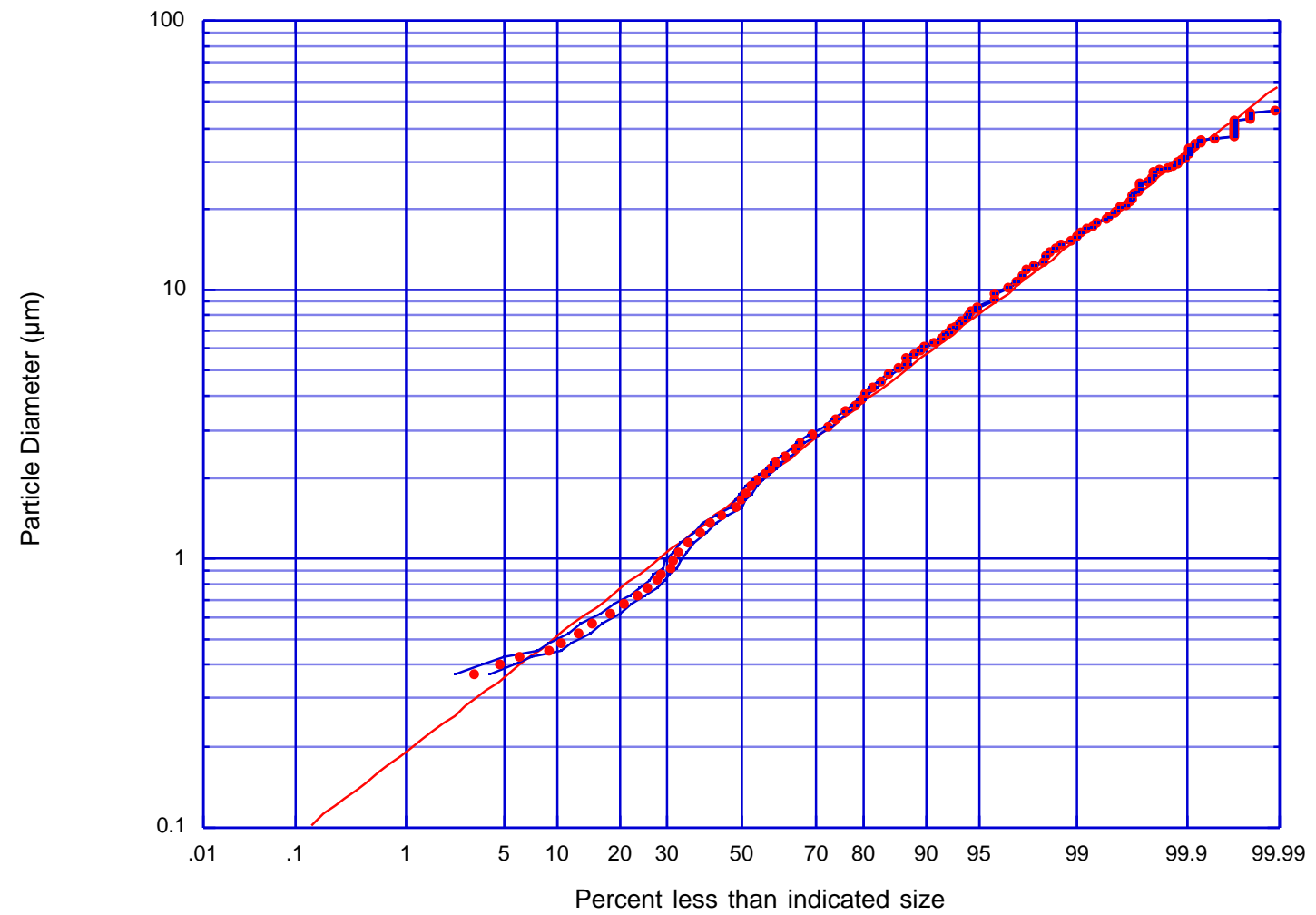

Analysis Summary Table:

\begin{tabular}{|c|c|c|c|c|c|}
\hline \multicolumn{2}{|c|}{$\begin{array}{c}\text { magnification, } \\
\text { number of images }\end{array}$} & \multicolumn{2}{|c|}{ area analyzed $^{1}(\%)$} & counted size range $(\mu \mathrm{m})$ & \multirow{2}{*}{$\begin{array}{c}\begin{array}{c}\text { total number of } \\
\text { particles (not scaled) }\end{array} \\
672\end{array}$} \\
\hline $50 x$ & 6 & \multicolumn{2}{|r|}{0.894} & $\mathrm{~d}_{\mathrm{p}}>8.46$ & \\
\hline $100 x$ & 6 & \multicolumn{2}{|r|}{0.224} & $4.44<\mathrm{d}_{\mathrm{p}}<8.46$ & 1145 \\
\hline $200 x$ & 6 & \multicolumn{2}{|r|}{0.0558} & $2.32<\mathrm{d}_{\mathrm{p}}<4.44$ & 1023 \\
\hline $500 x$ & 6 & \multicolumn{2}{|r|}{0.00893} & $1.21<\mathrm{d}_{\mathrm{p}}<2.32$ & 584 \\
\hline 1000x, & 6 & \multicolumn{2}{|r|}{0.00217} & $0.00<\mathrm{d}_{\mathrm{p}}<1.21$ & 227 \\
\hline \multicolumn{2}{|c|}{ Totals: } & \multicolumn{2}{|r|}{$1.18 \%$} & - & 3651 \\
\hline \multicolumn{4}{|c|}{ data moments ${ }^{2}:$} & \multicolumn{2}{|c|}{ log-normal fit moments ${ }^{2}$ : } \\
\hline \multicolumn{3}{|c|}{ GMD $(\mu \mathrm{m}):$} & 1.77 & GMD $(\mu \mathrm{m}):$ & 1.71 \\
\hline \multicolumn{2}{|c|}{ GSD: } & & 2.58 & GSD: & 2.57 \\
\hline \multicolumn{3}{|c|}{ lower $95 \%(\mu \mathrm{m})$ : } & 0.27 & $\mathrm{R}^{2}:$ & 0.99738 \\
\hline \multicolumn{3}{|c|}{ upper $95 \%(\mu \mathrm{m})$ : } & 11.80 & lower $95 \%(\mu \mathrm{m})$ : & 0.26 \\
\hline & & & & upper $95 \%(\mu \mathrm{m})$ : & 11.33 \\
\hline
\end{tabular}

1. based on overall filter area of $1.59 \times 10^{9} \mu \mathrm{m}^{2}$

2. $\mathrm{GMD}=$ geometric mean diameter, $\mathrm{GSD}=$ geometric standard deviation, $\mathrm{R}=$ linear correlation coefficient

Figure 3.63 Module VI, Sample 51 (TSFH12-03) count-based particle size distribution. 


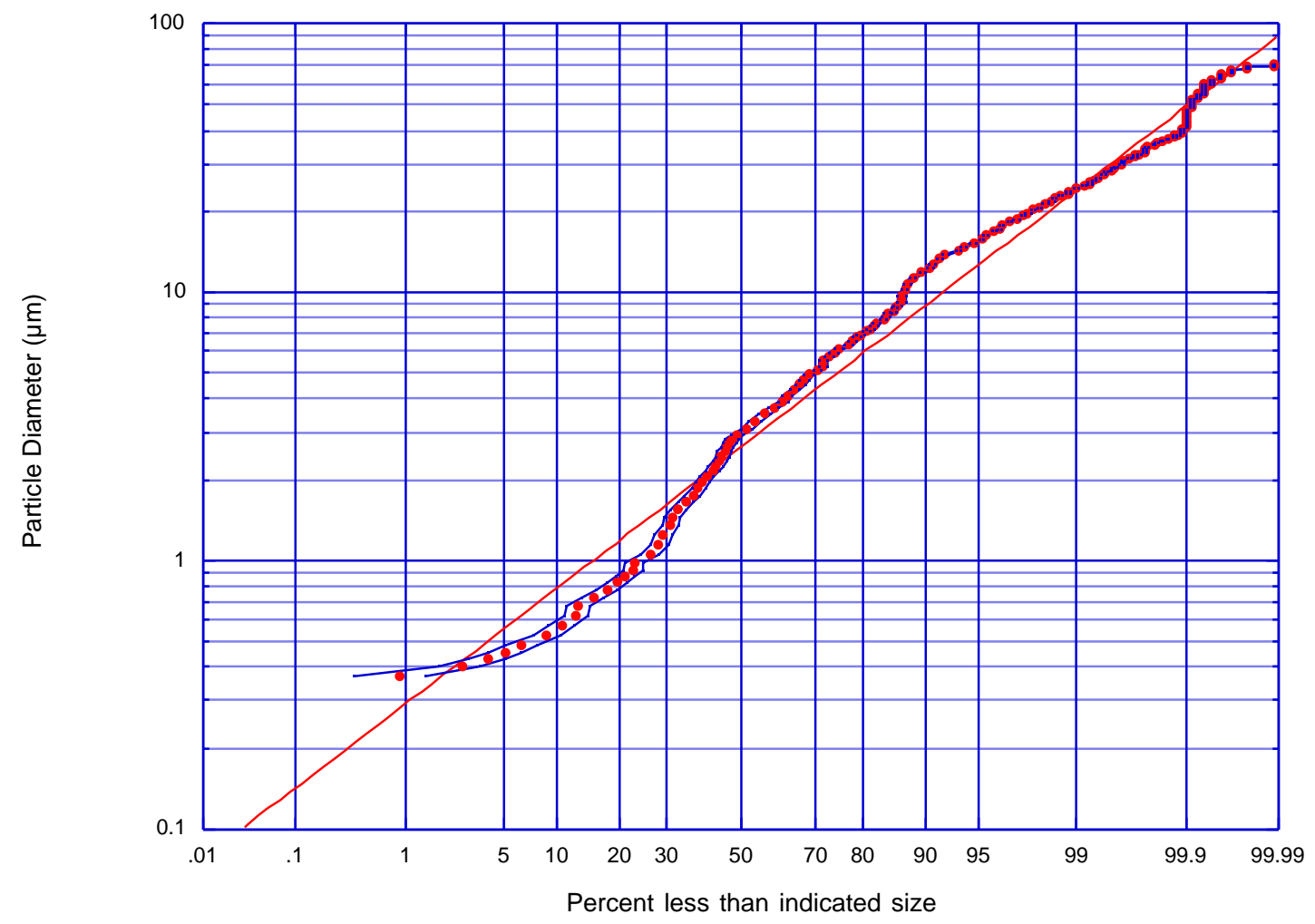

Analysis Summary Table:

\begin{tabular}{|c|c|c|c|c|c|}
\hline \multicolumn{2}{|c|}{$\begin{array}{c}\text { magnification, } \\
\text { number of images }\end{array}$} & \multicolumn{2}{|c|}{ area analyzed $^{1}(\%)$} & counted size range $(\mu \mathrm{m})$ & $\begin{array}{c}\text { total number of } \\
\text { particles (not scaled) }\end{array}$ \\
\hline $50 x$ & 6 & \multicolumn{2}{|r|}{0.894} & $d_{p}>8.93$ & 2958 \\
\hline $100 x$ & 6 & \multicolumn{2}{|r|}{0.224} & $5.17<\mathrm{d}_{\mathrm{p}}<8.93$ & 1828 \\
\hline $200 x$ & 6 & \multicolumn{2}{|r|}{0.0558} & $2.99<d_{p}<5.17$ & 1348 \\
\hline $500 x$ & 6 & \multicolumn{2}{|r|}{0.00893} & $1.78<\mathrm{d}_{\mathrm{p}}<2.99$ & 425 \\
\hline 1000x, & 6 & \multicolumn{2}{|r|}{0.00217} & $0.00<\mathrm{d}_{\mathrm{p}}<1.78$ & 259 \\
\hline \multicolumn{2}{|c|}{ Totals: } & \multicolumn{2}{|r|}{$1.18 \%$} & - & 6818 \\
\hline \multicolumn{4}{|c|}{ data moments ${ }^{2}$ : } & \multicolumn{2}{|c|}{ log-normal fit moments ${ }^{2}$ : } \\
\hline \multicolumn{3}{|c|}{ GMD $(\mu \mathrm{m}):$} & 2.72 & GMD $(\mu \mathrm{m}):$ & 2.65 \\
\hline \multicolumn{2}{|c|}{ GSD: } & & 2.99 & GSD: & 2.58 \\
\hline \multicolumn{3}{|c|}{ lower $95 \%(\mu \mathrm{m})$ : } & 0.30 & $\mathrm{R}^{2}$ & 0.98804 \\
\hline \multirow{2}{*}{\multicolumn{3}{|c|}{ upper $95 \%(\mu \mathrm{m})$ : }} & 24.29 & lower $95 \%(\mu \mathrm{m})$ : & 0.40 \\
\hline & & & & upper $95 \%(\mu \mathrm{m})$ : & 17.63 \\
\hline
\end{tabular}

1. based on overall filter area of $1.59 \times 10^{9} \mu \mathrm{m}^{2}$

2. $\mathrm{GMD}=$ geometric mean diameter, $\mathrm{GSD}=$ geometric standard deviation, $\mathrm{R}=$ linear correlation coefficient

Figure 3.64 Module VI, Sample 52 (TSFH17-03) count-based particle size distribution. 


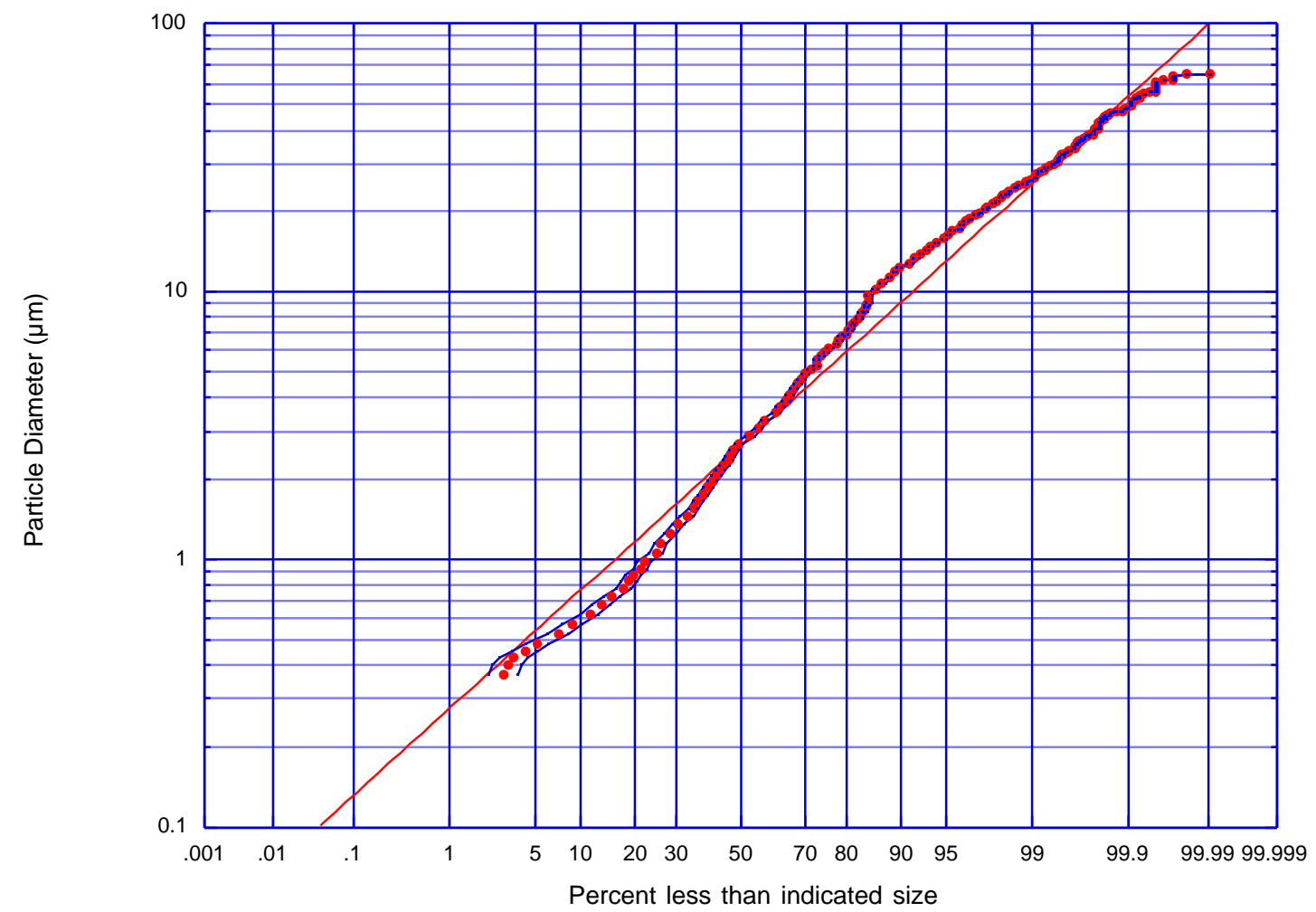

Analysis Summary Table:

\begin{tabular}{|c|c|c|c|c|c|}
\hline \multicolumn{2}{|c|}{$\begin{array}{c}\text { magnification, } \\
\text { number of images }\end{array}$} & \multicolumn{2}{|c|}{ area analyzed $^{1}(\%)$} & counted size range $(\mu \mathrm{m})$ & $\begin{array}{c}\text { total number of } \\
\text { particles (not scaled) }\end{array}$ \\
\hline $50 x$ & 6 & \multicolumn{2}{|r|}{0.894} & $d_{p}>9.15$ & 2959 \\
\hline $100 x$ & 6 & \multicolumn{2}{|r|}{0.224} & $4.98<d_{p}<9.15$ & 1825 \\
\hline $200 x$ & 6 & \multicolumn{2}{|r|}{0.0558} & $2.67<\mathrm{d}_{\mathrm{p}}<4.98$ & 1467 \\
\hline $500 x$ & 6 & \multicolumn{2}{|r|}{0.00893} & $1.56<d_{p}<2.67$ & 475 \\
\hline 1000x, & 6 & \multicolumn{2}{|r|}{0.00217} & $0.00<\mathrm{d}_{\mathrm{p}}<1.56$ & 283 \\
\hline \multicolumn{2}{|c|}{ Totals: } & \multicolumn{2}{|r|}{$1.18 \%$} & - & 7009 \\
\hline \multicolumn{4}{|c|}{ data moments ${ }^{2}:$} & \multicolumn{2}{|c|}{ log-normal fit moments ${ }^{2}$ : } \\
\hline \multicolumn{3}{|c|}{ GMD $(\mu \mathrm{m}):$} & 2.69 & GMD $(\mu \mathrm{m}):$ & 2.65 \\
\hline \multicolumn{2}{|c|}{ GSD: } & & 2.99 & GSD: & 2.64 \\
\hline \multicolumn{3}{|c|}{ lower $95 \%(\mu \mathrm{m})$ : } & 0.30 & $\mathrm{R}^{2}$ & 0.98883 \\
\hline \multirow{2}{*}{\multicolumn{3}{|c|}{ upper $95 \%(\mu \mathrm{m})$ : }} & 24.10 & lower $95 \%(\mu \mathrm{m})$ : & 0.38 \\
\hline & & & & upper $95 \%(\mu \mathrm{m})$ : & 18.54 \\
\hline
\end{tabular}

1. based on overall filter area of $1.59 \times 10^{9} \mu \mathrm{m}^{2}$

2. $\mathrm{GMD}=$ geometric mean diameter, $\mathrm{GSD}=$ geometric standard deviation, $\mathrm{R}=$ linear correlation coefficient

Figure 3.65 Module VI, Sample 53 (TSFH13-03) count-based particle size distribution. 


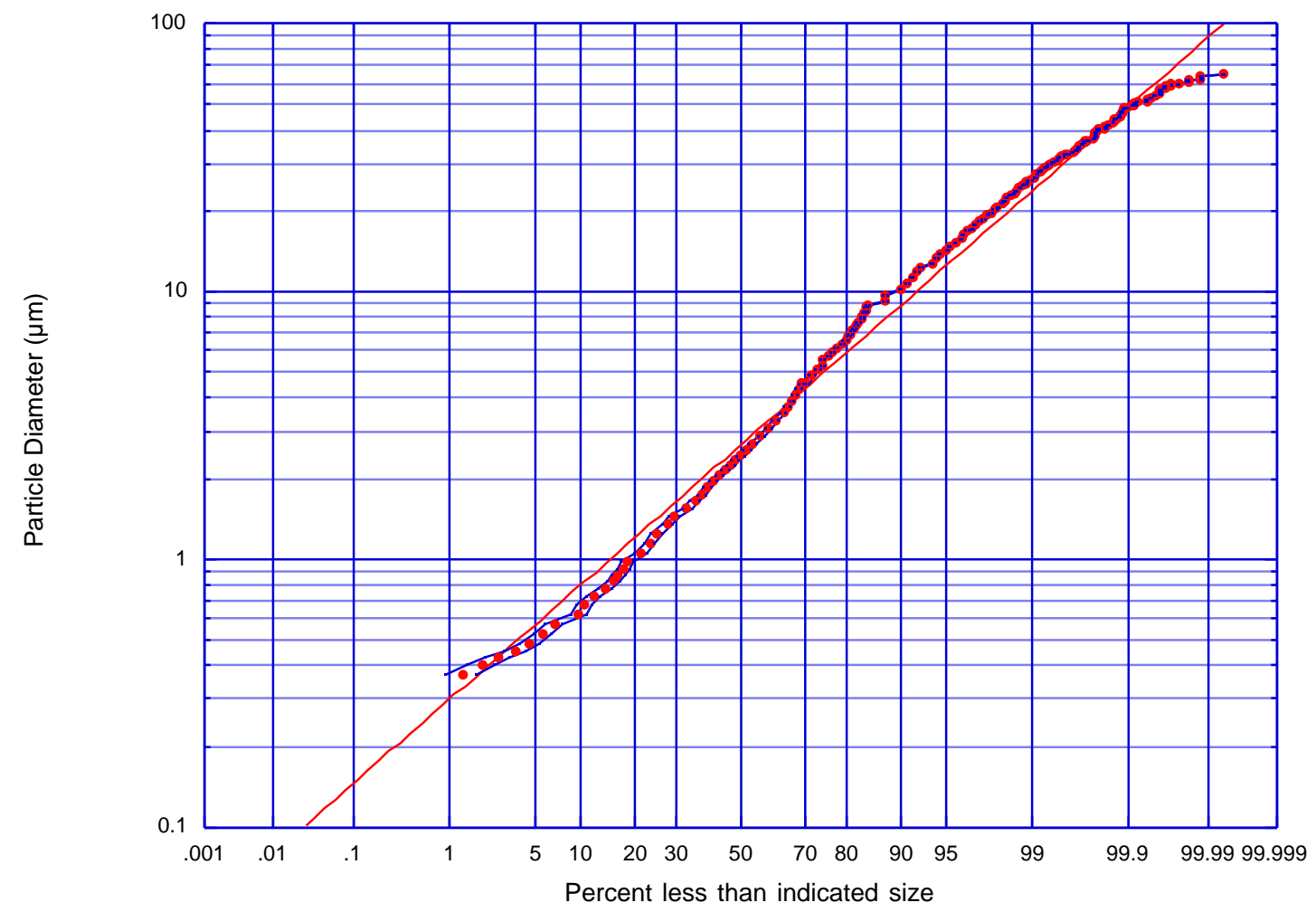

Analysis Summary Table:

\begin{tabular}{|c|c|c|c|c|c|}
\hline \multicolumn{2}{|c|}{$\begin{array}{c}\text { magnification, } \\
\text { number of images }\end{array}$} & \multicolumn{2}{|c|}{ area analyzed $^{1}(\%)$} & counted size range $(\mu \mathrm{m})$ & $\begin{array}{c}\text { total number of } \\
\text { particles (not scaled) }\end{array}$ \\
\hline $50 x$ & 6 & \multicolumn{2}{|r|}{0.894} & $d_{p}>9.04$ & 3325 \\
\hline $100 x$ & 6 & \multicolumn{2}{|r|}{0.224} & $4.76<d_{p}<9.04$ & 2542 \\
\hline $200 x$ & 6 & \multicolumn{2}{|r|}{0.0558} & $2.55<d_{p}<4.76$ & 1820 \\
\hline $500 x$ & 6 & \multicolumn{2}{|r|}{0.00893} & $1.53<\mathrm{d}_{\mathrm{p}}<2.55$ & 1007 \\
\hline 1000x, & 6 & \multicolumn{2}{|r|}{0.00217} & $0.00<\mathrm{d}_{\mathrm{p}}<1.53$ & 380 \\
\hline \multicolumn{2}{|c|}{ Totals: } & \multicolumn{2}{|r|}{$1.18 \%$} & - & 9074 \\
\hline \multicolumn{4}{|c|}{ data moments ${ }^{2}:$} & \multicolumn{2}{|c|}{ log-normal fit moments ${ }^{2}$ : } \\
\hline \multicolumn{3}{|c|}{ GMD $(\mu \mathrm{m}):$} & 2.66 & GMD $(\mu \mathrm{m}):$ & 2.67 \\
\hline \multicolumn{2}{|c|}{ GSD: } & & 2.78 & GSD: & 2.56 \\
\hline \multicolumn{3}{|c|}{ lower $95 \%(\mu \mathrm{m})$ : } & 0.35 & $\mathrm{R}^{2}$ & 0.99168 \\
\hline \multirow{2}{*}{\multicolumn{3}{|c|}{ upper $95 \%(\mu \mathrm{m})$ : }} & 20.55 & lower $95 \%(\mu \mathrm{m})$ : & 0.41 \\
\hline & & & & upper $95 \%(\mu \mathrm{m})$ : & 17.58 \\
\hline
\end{tabular}

1. based on overall filter area of $1.59 \times 10^{9} \mu \mathrm{m}^{2}$

2. $\mathrm{GMD}=$ geometric mean diameter, $\mathrm{GSD}=$ geometric standard deviation, $\mathrm{R}=$ linear correlation coefficient

Figure 3.66 Module VI, Sample 54 (TSFH27-02) count-based particle size distribution. 


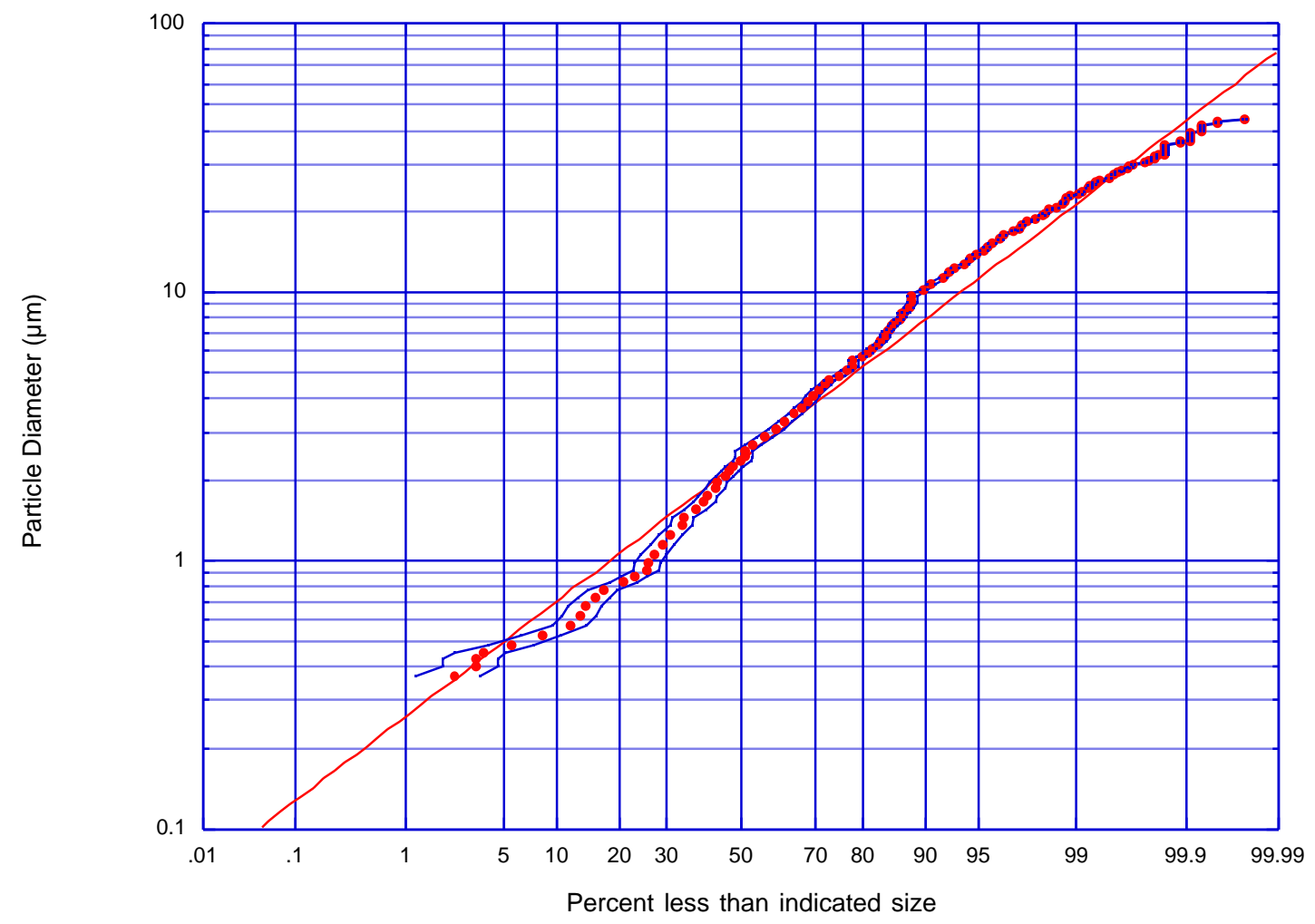

Analysis Summary Table:

\begin{tabular}{|c|c|c|c|c|c|}
\hline \multicolumn{2}{|c|}{$\begin{array}{c}\text { magnification, } \\
\text { number of images }\end{array}$} & \multicolumn{2}{|c|}{ area analyzed $^{1}(\%)$} & counted size range $(\mu \mathrm{m})$ & $\begin{array}{c}\text { total number of } \\
\text { particles (not scaled) }\end{array}$ \\
\hline $50 x$ & 6 & \multicolumn{2}{|r|}{0.894} & $d_{p}>8.85$ & 902 \\
\hline $100 x$ & 6 & \multicolumn{2}{|r|}{0.224} & $4.81<\mathrm{d}_{\mathrm{p}}<8.85$ & 666 \\
\hline $200 x$ & 6 & \multicolumn{2}{|r|}{0.0558} & $2.64<d_{p}<4.81$ & 572 \\
\hline $500 x$ & 6 & \multicolumn{2}{|r|}{0.00893} & $1.51<\mathrm{d}_{\mathrm{p}}<2.64$ & 293 \\
\hline 1000x, & 6 & \multicolumn{2}{|r|}{0.00217} & $0.00<\mathrm{d}_{\mathrm{p}}<1.51$ & 109 \\
\hline \multicolumn{2}{|c|}{ Totals: } & \multicolumn{2}{|r|}{$1.18 \%$} & - & 2542 \\
\hline \multicolumn{4}{|c|}{ data moments ${ }^{2}:$} & \multicolumn{2}{|c|}{ log-normal fit moments ${ }^{2}$ : } \\
\hline \multicolumn{3}{|c|}{ GMD $(\mu \mathrm{m}):$} & 2.39 & GMD $(\mu \mathrm{m}):$ & 2.36 \\
\hline \multicolumn{2}{|c|}{ GSD: } & & 2.85 & GSD: & 2.58 \\
\hline \multicolumn{3}{|c|}{ lower $95 \%(\mu \mathrm{m})$ : } & 0.30 & $\mathrm{R}^{2}$ & 0.98625 \\
\hline \multirow{2}{*}{\multicolumn{3}{|c|}{ upper $95 \%(\mu \mathrm{m})$ : }} & 19.37 & lower $95 \%(\mu \mathrm{m})$ : & 0.36 \\
\hline & & & & upper $95 \%(\mu \mathrm{m})$ : & 15.66 \\
\hline
\end{tabular}

1. based on overall filter area of $1.59 \times 10^{9} \mu \mathrm{m}^{2}$

2. $\mathrm{GMD}=$ geometric mean diameter, $\mathrm{GSD}=$ geometric standard deviation, $\mathrm{R}=$ linear correlation coefficient

Figure 3.67 Module VI, Sample 55 (TSFH26-03) count-based particle size distribution. 


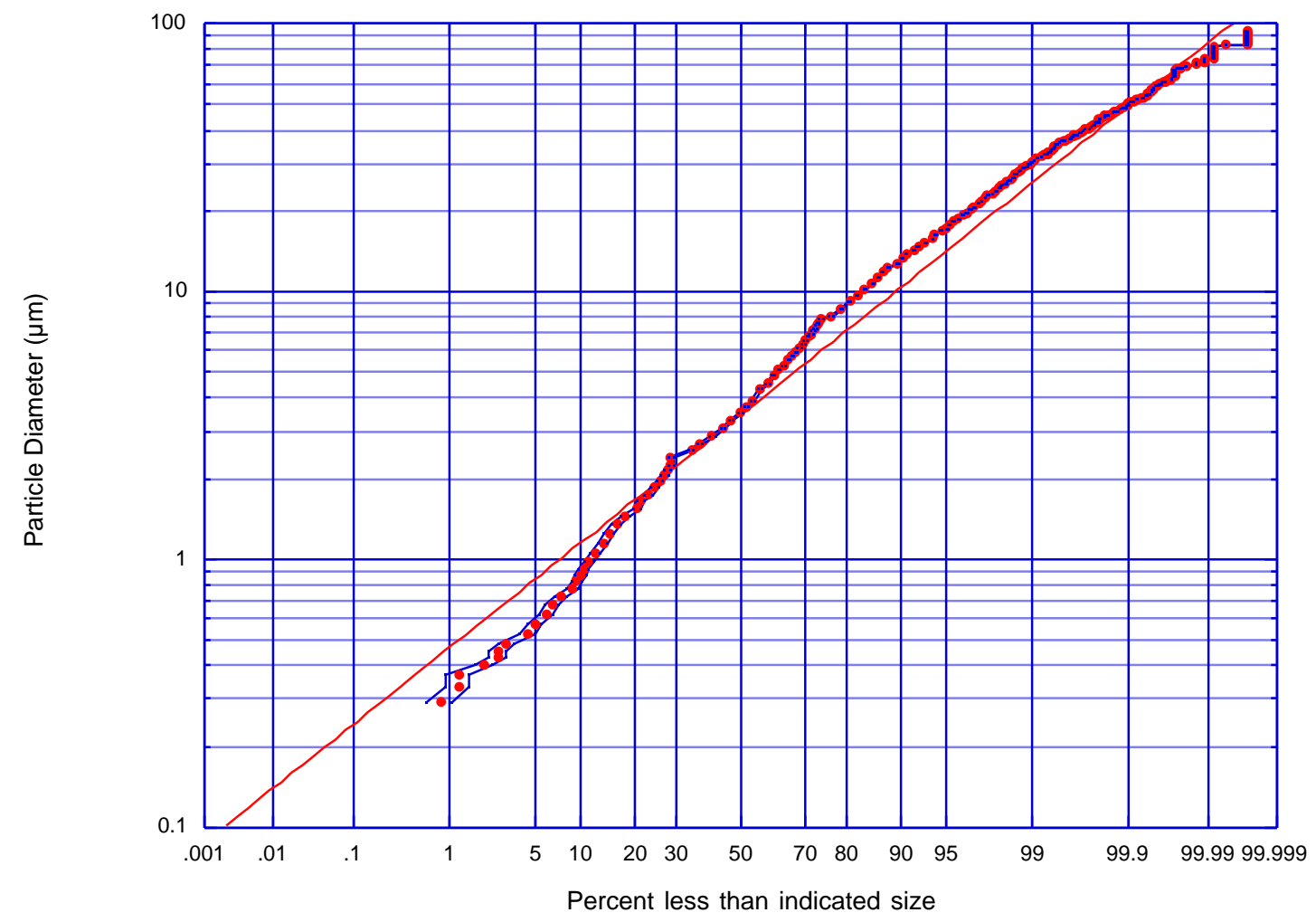

Analysis Summary Table:

\begin{tabular}{|c|c|c|c|c|c|}
\hline \multicolumn{2}{|c|}{$\begin{array}{c}\text { magnification, } \\
\text { number of images }\end{array}$} & \multicolumn{2}{|c|}{ area analyzed $^{1}(\%)$} & counted size range $(\mu \mathrm{m})$ & $\begin{array}{c}\text { total number of } \\
\text { particles (not scaled) }\end{array}$ \\
\hline $50 x$ & 6 & \multicolumn{2}{|r|}{0.894} & $d_{p}>7.91$ & 13622 \\
\hline $100 x$ & 6 & \multicolumn{2}{|r|}{0.224} & $4.25<d_{p}<7.91$ & 8158 \\
\hline $200 x$ & 6 & \multicolumn{2}{|r|}{0.0558} & $2.30<d_{p}<4.25$ & 6069 \\
\hline $500 x$ & 6 & \multicolumn{2}{|r|}{0.00893} & $1.37<\mathrm{d}_{\mathrm{p}}<2.30$ & 1635 \\
\hline 1000x, & 6 & \multicolumn{2}{|r|}{0.00217} & $0.00<\mathrm{d}_{\mathrm{p}}<1.37$ & 448 \\
\hline \multicolumn{2}{|c|}{ Totals: } & \multicolumn{2}{|r|}{$1.18 \%$} & - & 29932 \\
\hline \multicolumn{4}{|c|}{ data moments ${ }^{2}:$} & \multicolumn{2}{|c|}{ log-normal fit moments ${ }^{2}$ : } \\
\hline \multicolumn{3}{|c|}{ GMD $(\mu \mathrm{m}):$} & 3.71 & GMD $(\mu \mathrm{m}):$ & 3.46 \\
\hline \multicolumn{2}{|c|}{ GSD: } & & 2.75 & GSD: & 2.36 \\
\hline \multicolumn{3}{|c|}{ lower $95 \%(\mu \mathrm{m})$ : } & 0.49 & $\mathrm{R}^{2}$ & 0.98707 \\
\hline \multirow{2}{*}{\multicolumn{3}{|c|}{ upper $95 \%(\mu \mathrm{m})$ : }} & 28.01 & lower $95 \%(\mu \mathrm{m})$ : & 0.62 \\
\hline & & & & upper $95 \%(\mu \mathrm{m})$ : & 19.33 \\
\hline
\end{tabular}

1. based on overall filter area of $1.59 \times 10^{9} \mu \mathrm{m}^{2}$

2. $\mathrm{GMD}=$ geometric mean diameter, $\mathrm{GSD}=$ geometric standard deviation, $\mathrm{R}=$ linear correlation coefficient

Figure 3.68 Module VI, Sample 56 (TSFH27-01) count-based particle size distribution. 


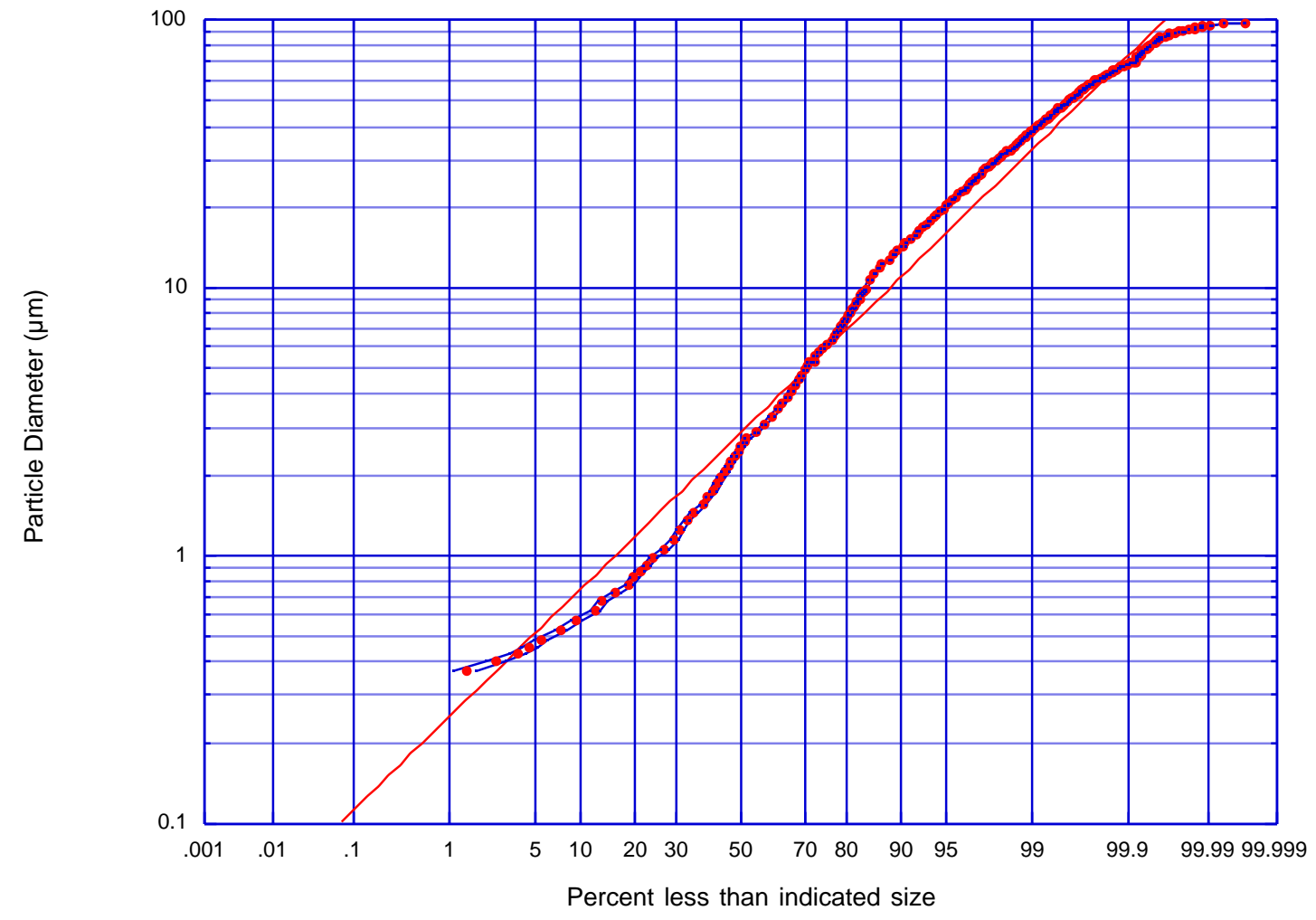

Analysis Summary Table:

\begin{tabular}{|c|c|c|c|c|c|}
\hline \multicolumn{2}{|c|}{$\begin{array}{c}\text { magnification, } \\
\text { number of images }\end{array}$} & \multicolumn{2}{|c|}{ area analyzed $^{1}(\%)$} & counted size range $(\mu \mathrm{m})$ & $\begin{array}{c}\text { total number of } \\
\text { particles (not scaled) }\end{array}$ \\
\hline $50 x$ & 6 & \multicolumn{2}{|r|}{0.894} & $d_{p}>10.50$ & 8590 \\
\hline $100 x$ & 6 & \multicolumn{2}{|r|}{0.224} & $5.44<\mathrm{d}_{\mathrm{p}}<10.50$ & 5468 \\
\hline $200 x$ & 6 & \multicolumn{2}{|r|}{0.0558} & $2.81<\mathrm{d}_{\mathrm{p}}<5.44$ & 4041 \\
\hline $500 x$ & 6 & \multicolumn{2}{|r|}{0.00893} & $1.56<\mathrm{d}_{\mathrm{p}}<2.81$ & 1775 \\
\hline 1000x, & 6 & \multicolumn{2}{|r|}{0.00217} & $0.00<\mathrm{d}_{\mathrm{p}}<1.56$ & 870 \\
\hline \multicolumn{2}{|c|}{ Totals: } & \multicolumn{2}{|r|}{$1.18 \%$} & - & 20744 \\
\hline \multicolumn{4}{|c|}{ data moments ${ }^{2}:$} & \multicolumn{2}{|c|}{ log-normal fit moments ${ }^{2}$ : } \\
\hline \multicolumn{3}{|c|}{ GMD $(\mu \mathrm{m}):$} & 2.69 & GMD $(\mu \mathrm{m}):$ & 2.87 \\
\hline \multicolumn{2}{|c|}{ GSD: } & & 3.23 & GSD: & 2.86 \\
\hline \multicolumn{3}{|c|}{ lower $95 \%(\mu \mathrm{m})$ : } & 0.26 & $\mathrm{R}^{2}$ & 0.98367 \\
\hline \multirow{2}{*}{\multicolumn{3}{|c|}{ upper $95 \%(\mu \mathrm{m})$ : }} & 28.06 & lower $95 \%(\mu \mathrm{m})$ : & 0.35 \\
\hline & & & & upper $95 \%(\mu \mathrm{m})$ : & 23.50 \\
\hline
\end{tabular}

1. based on overall filter area of $1.59 \times 10^{9} \mu \mathrm{m}^{2}$

2. $\mathrm{GMD}=$ geometric mean diameter, $\mathrm{GSD}=$ geometric standard deviation, $\mathrm{R}=$ linear correlation coefficient

Figure 3.69 Module VI, Sample 57 (TSFH14-03) count-based particle size distribution. 


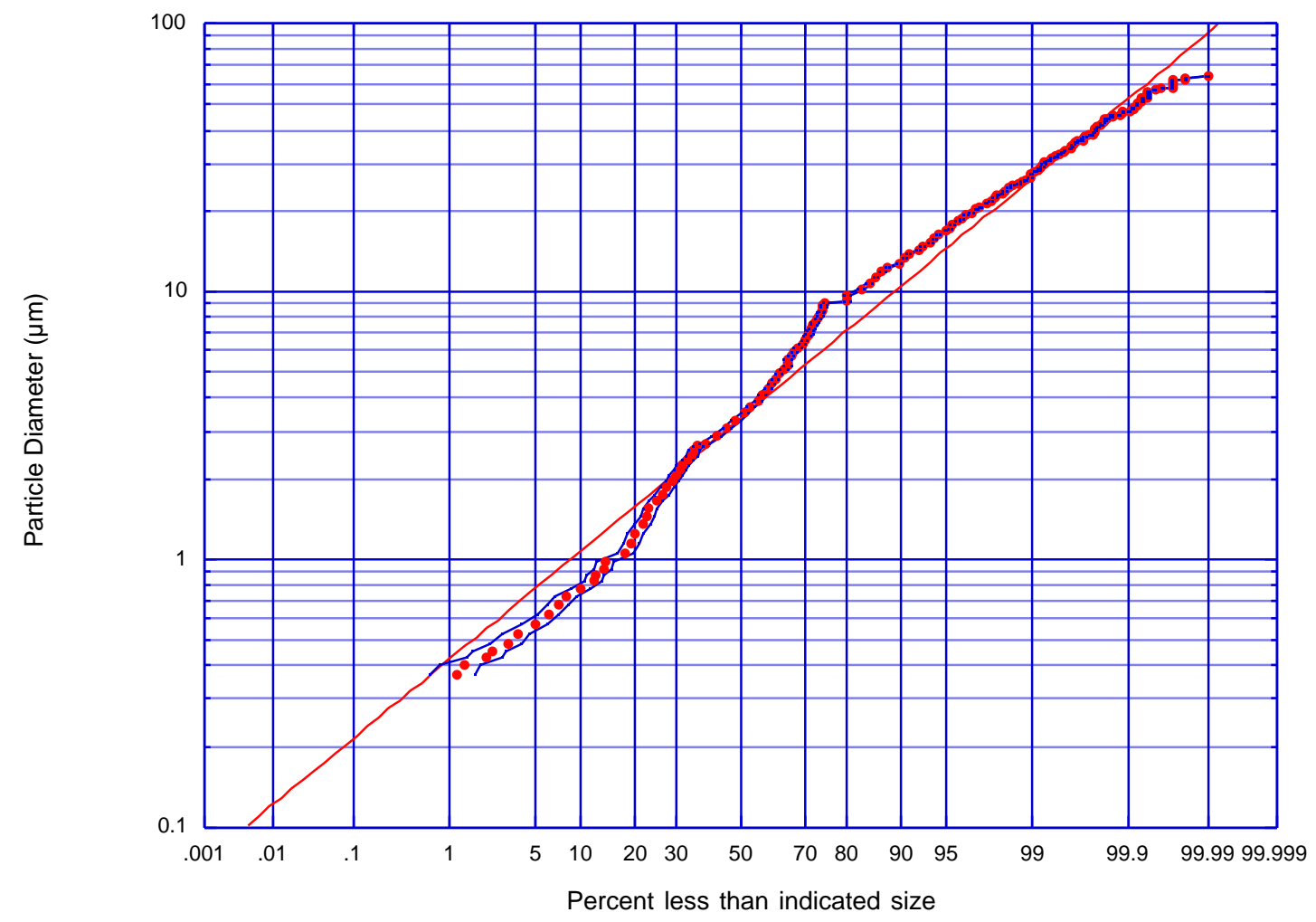

Analysis Summary Table:

\begin{tabular}{|c|c|c|c|c|c|}
\hline \multicolumn{2}{|c|}{$\begin{array}{c}\text { magnification, } \\
\text { number of images }\end{array}$} & \multicolumn{2}{|c|}{ area analyzed $^{1}(\%)$} & counted size range $(\mu \mathrm{m})$ & $\begin{array}{c}\text { total number of } \\
\text { particles (not scaled) }\end{array}$ \\
\hline $50 x$ & 6 & \multicolumn{2}{|r|}{0.894} & $d_{p}>9.19$ & 3220 \\
\hline $100 x$ & 6 & \multicolumn{2}{|r|}{0.224} & $5.07<\mathrm{d}_{\mathrm{p}}<9.19$ & 1563 \\
\hline $200 x$ & 6 & \multicolumn{2}{|r|}{0.0558} & $2.78<d_{p}<5.07$ & 1568 \\
\hline $500 x$ & 6 & \multicolumn{2}{|r|}{0.00893} & $1.69<d_{p}<2.78$ & 554 \\
\hline 1000x, & 6 & \multicolumn{2}{|r|}{0.00217} & $0.00<\mathrm{d}_{\mathrm{p}}<1.69$ & 203 \\
\hline \multicolumn{2}{|c|}{ Totals: } & \multicolumn{2}{|r|}{$1.18 \%$} & - & 7108 \\
\hline \multicolumn{4}{|c|}{ data moments ${ }^{2}:$} & \multicolumn{2}{|c|}{ log-normal fit moments ${ }^{2}$ : } \\
\hline \multicolumn{3}{|c|}{ GMD $(\mu \mathrm{m}):$} & 3.53 & GMD $(\mu \mathrm{m}):$ & 3.36 \\
\hline \multicolumn{2}{|c|}{ GSD: } & & 2.84 & GSD: & 2.44 \\
\hline \multicolumn{3}{|c|}{ lower $95 \%(\mu \mathrm{m})$ : } & 0.44 & $\mathrm{R}^{2}$ & 0.98551 \\
\hline \multirow{2}{*}{\multicolumn{3}{|c|}{ upper $95 \%(\mu \mathrm{m})$ : }} & 28.57 & lower $95 \%(\mu \mathrm{m})$ : & 0.57 \\
\hline & & & & upper $95 \%(\mu \mathrm{m})$ : & 19.94 \\
\hline
\end{tabular}

1. based on overall filter area of $1.59 \times 10^{9} \mu \mathrm{m}^{2}$

2. $\mathrm{GMD}=$ geometric mean diameter, $\mathrm{GSD}=$ geometric standard deviation, $\mathrm{R}=$ linear correlation coefficient

Figure 3.70 Module VI, Sample 58 (TSFH26-02) count-based particle size distribution. 


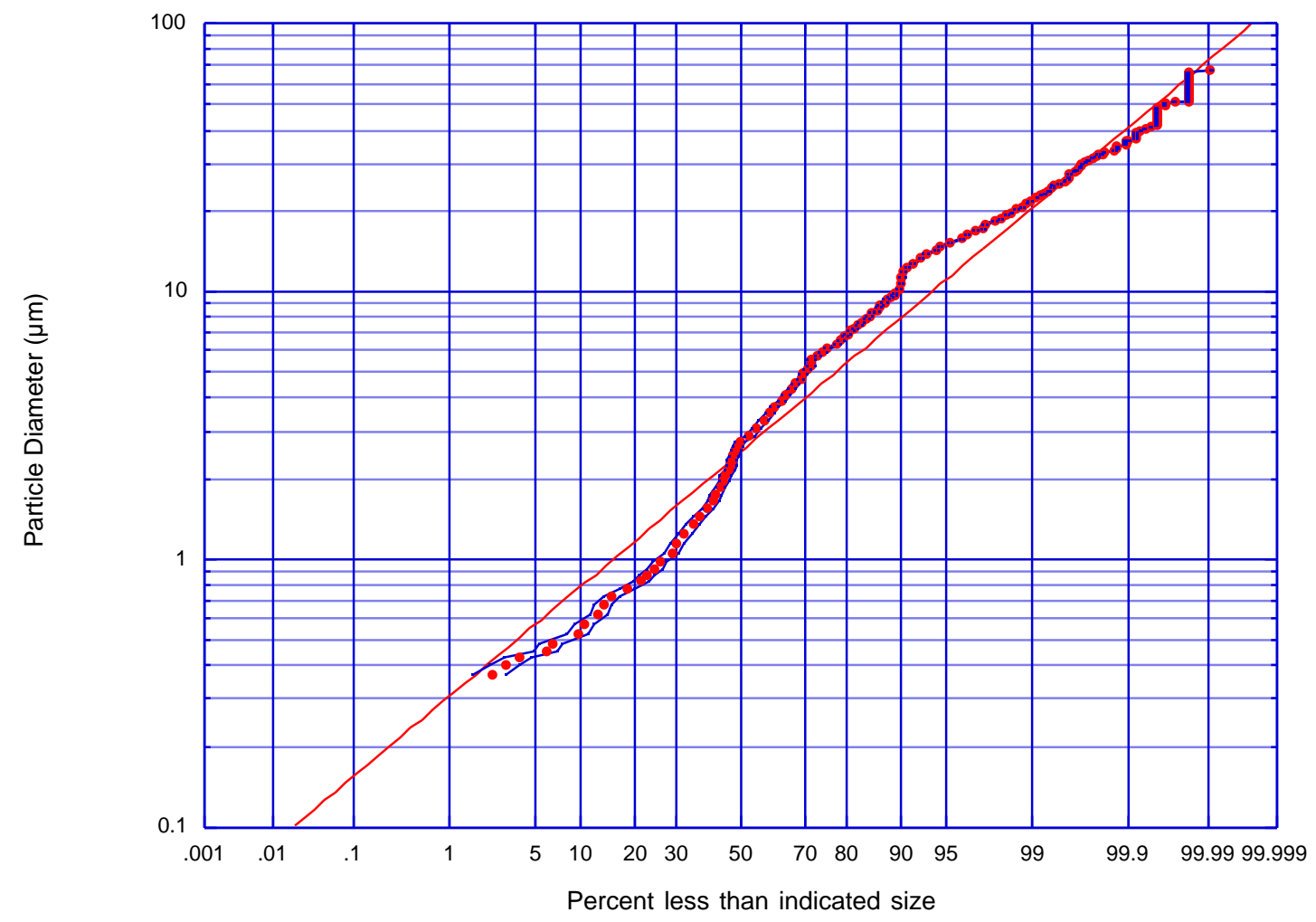

Analysis Summary Table:

\begin{tabular}{|c|c|c|c|c|c|}
\hline \multicolumn{2}{|c|}{$\begin{array}{c}\text { magnification, } \\
\text { number of images }\end{array}$} & \multicolumn{2}{|c|}{ area analyzed $^{1}(\%)$} & counted size range $(\mu \mathrm{m})$ & $\begin{array}{c}\text { total number of } \\
\text { particles (not scaled) }\end{array}$ \\
\hline $50 x$ & 6 & \multicolumn{2}{|r|}{0.894} & $d_{p}>8.60$ & 3959 \\
\hline $100 x$ & 6 & \multicolumn{2}{|r|}{0.224} & $4.97<\mathrm{d}_{\mathrm{p}}<8.60$ & 2263 \\
\hline $200 x$ & 6 & \multicolumn{2}{|r|}{0.0558} & $2.80<\mathrm{d}_{\mathrm{p}}<4.97$ & 1397 \\
\hline $500 x$ & 6 & \multicolumn{2}{|r|}{0.00893} & $1.53<\mathrm{d}_{\mathrm{p}}<2.80$ & 509 \\
\hline 1000x, & 6 & \multicolumn{2}{|r|}{0.00217} & $0.00<\mathrm{d}_{\mathrm{p}}<1.53$ & 289 \\
\hline \multicolumn{2}{|c|}{ Totals: } & \multicolumn{2}{|r|}{$1.18 \%$} & - & 8417 \\
\hline \multicolumn{4}{|c|}{ data moments ${ }^{2}$ : } & \multicolumn{2}{|c|}{ log-normal fit moments ${ }^{2}$ : } \\
\hline \multicolumn{3}{|c|}{ GMD $(\mu \mathrm{m}):$} & 2.52 & GMD $(\mu \mathrm{m}):$ & 2.52 \\
\hline \multicolumn{2}{|c|}{ GSD: } & & 3.00 & GSD: & 2.47 \\
\hline \multicolumn{3}{|c|}{ lower $95 \%(\mu \mathrm{m})$ : } & 0.28 & $\mathrm{R}^{2}$ & 0.98526 \\
\hline \multirow{2}{*}{\multicolumn{3}{|c|}{ upper $95 \%(\mu \mathrm{m})$ : }} & 22.67 & lower $95 \%(\mu \mathrm{m})$ : & 0.41 \\
\hline & & & & upper $95 \%(\mu \mathrm{m})$ : & 15.35 \\
\hline
\end{tabular}

1. based on overall filter area of $1.59 \times 10^{9} \mu \mathrm{m}^{2}$

2. $\mathrm{GMD}=$ geometric mean diameter, $\mathrm{GSD}=$ geometric standard deviation, $\mathrm{R}=$ linear correlation coefficient

Figure 3.71 Module VI, Sample 59 (TSFH19-03) count-based particle size distribution. 


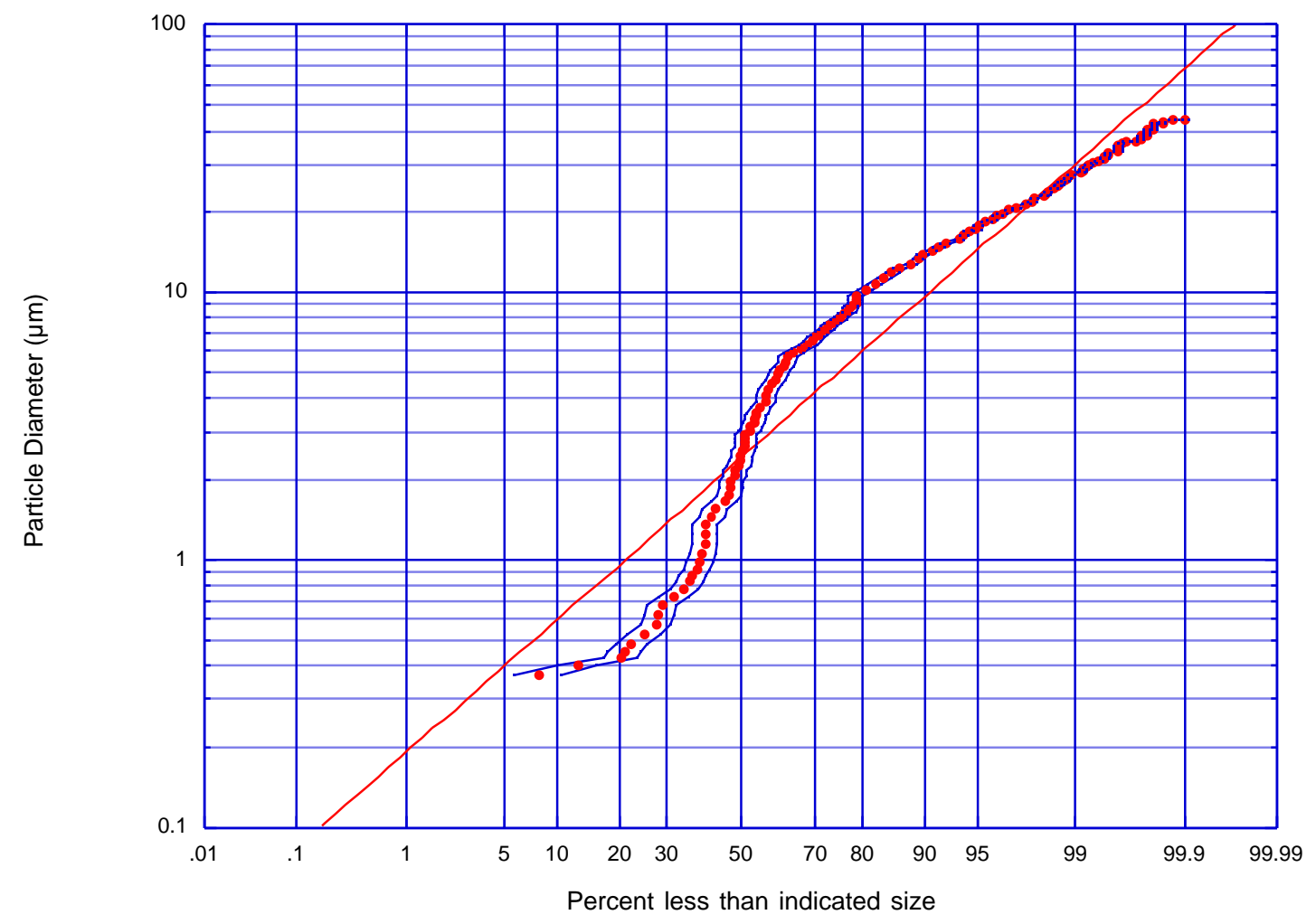

Analysis Summary Table:

\begin{tabular}{|c|c|c|c|c|c|}
\hline \multicolumn{2}{|c|}{$\begin{array}{c}\text { magnification, } \\
\text { number of images }\end{array}$} & \multicolumn{2}{|c|}{ area analyzed $^{1}(\%)$} & counted size range $(\mu \mathrm{m})$ & $\begin{array}{c}\text { total number of } \\
\text { particles (not scaled) }\end{array}$ \\
\hline $50 x$ & 6 & \multicolumn{2}{|r|}{0.894} & $d_{p}>9.41$ & 1082 \\
\hline $100 x$ & 6 & \multicolumn{2}{|r|}{0.224} & $5.70<d_{p}<9.41$ & 621 \\
\hline $200 x$ & 6 & \multicolumn{2}{|r|}{0.0558} & $3.63<d_{p}<5.70$ & 237 \\
\hline $500 x$ & 6 & \multicolumn{2}{|r|}{0.00893} & $1.96<\mathrm{d}_{\mathrm{p}}<3.63$ & 84 \\
\hline 1000x, & 6 & \multicolumn{2}{|r|}{0.00217} & $0.00<\mathrm{d}_{\mathrm{p}}<1.96$ & 94 \\
\hline \multicolumn{2}{|c|}{ Totals: } & \multicolumn{2}{|r|}{$1.18 \%$} & - & 2118 \\
\hline \multicolumn{4}{|c|}{ data moments ${ }^{2}:$} & \multicolumn{2}{|c|}{ log-normal fit moments ${ }^{2}$ : } \\
\hline \multicolumn{3}{|c|}{ GMD $(\mu \mathrm{m}):$} & 2.35 & GMD $(\mu \mathrm{m}):$ & 2.40 \\
\hline \multicolumn{2}{|c|}{ GSD: } & & 4.03 & GSD: & 2.97 \\
\hline \multicolumn{3}{|c|}{ lower $95 \%(\mu \mathrm{m})$ : } & 0.14 & $\mathrm{R}^{2}$ & 0.92636 \\
\hline \multirow{2}{*}{\multicolumn{3}{|c|}{ upper $95 \%(\mu \mathrm{m})$ : }} & 38.19 & lower $95 \%(\mu \mathrm{m})$ : & 0.27 \\
\hline & & & & upper $95 \%(\mu \mathrm{m})$ : & 21.15 \\
\hline
\end{tabular}

1. based on overall filter area of $1.59 \times 10^{9} \mu \mathrm{m}^{2}$

2. $\mathrm{GMD}=$ geometric mean diameter, $\mathrm{GSD}=$ geometric standard deviation, $\mathrm{R}=$ linear correlation coefficient

Figure 3.72 Module VI, Sample 60 (TSFH15-03) count-based particle size distribution. 


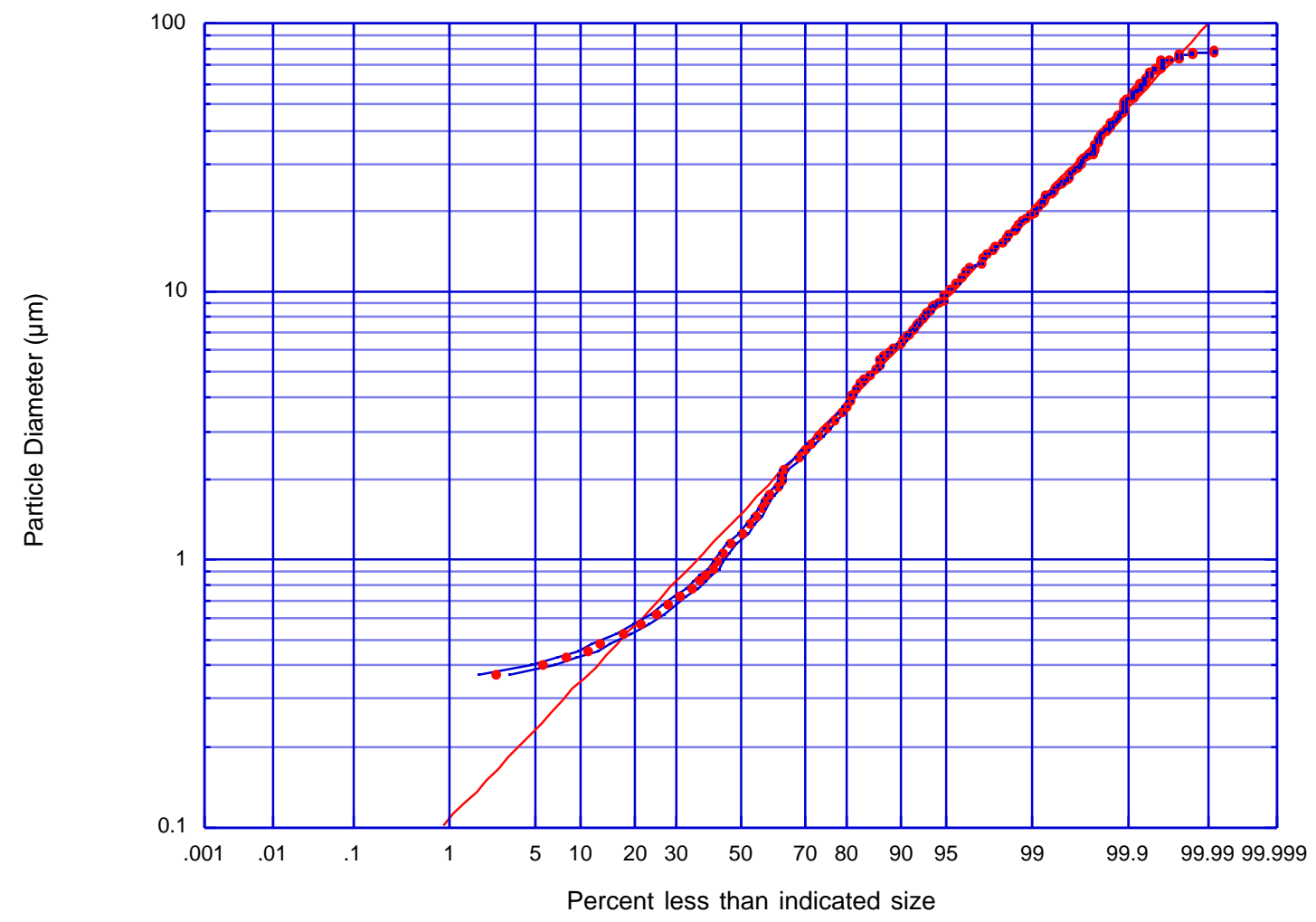

Analysis Summary Table:

\begin{tabular}{|c|c|c|c|c|c|}
\hline \multicolumn{2}{|c|}{$\begin{array}{c}\text { magnification, } \\
\text { number of images }\end{array}$} & \multicolumn{2}{|c|}{ area analyzed $^{1}(\%)$} & counted size range $(\mu \mathrm{m})$ & $\begin{array}{c}\text { total number of } \\
\text { particles (not scaled) }\end{array}$ \\
\hline $50 x$ & 6 & \multicolumn{2}{|r|}{0.894} & $d_{p}>9.13$ & 1177 \\
\hline $100 x$ & 6 & \multicolumn{2}{|r|}{0.224} & $4.84<d_{p}<9.13$ & 1354 \\
\hline $200 x$ & 6 & \multicolumn{2}{|r|}{0.0558} & $2.34<\mathrm{d}_{\mathrm{p}}<4.84$ & 1091 \\
\hline $500 x$ & 6 & \multicolumn{2}{|r|}{0.00893} & $1.15<\mathrm{d}_{\mathrm{p}}<2.34$ & 548 \\
\hline 1000x, & 6 & \multicolumn{2}{|r|}{0.00217} & $0.00<\mathrm{d}_{\mathrm{p}}<1.15$ & 416 \\
\hline \multicolumn{2}{|c|}{ Totals: } & \multicolumn{2}{|r|}{$1.18 \%$} & - & 4586 \\
\hline \multicolumn{4}{|c|}{ data moments ${ }^{2}:$} & \multicolumn{2}{|c|}{ log-normal fit moments ${ }^{2}$ : } \\
\hline \multicolumn{3}{|c|}{ GMD $(\mu \mathrm{m}):$} & 1.54 & GMD $(\mu \mathrm{m}):$ & 1.48 \\
\hline \multicolumn{2}{|c|}{ GSD: } & & 2.79 & GSD: & 3.10 \\
\hline \multicolumn{3}{|c|}{ lower $95 \%(\mu \mathrm{m})$ : } & 0.20 & $\mathrm{R}^{2}$ & 0.99461 \\
\hline \multirow{2}{*}{\multicolumn{3}{|c|}{ upper $95 \%(\mu \mathrm{m})$ : }} & 11.93 & lower $95 \%(\mu \mathrm{m})$ : & 0.15 \\
\hline & & & & upper $95 \%(\mu \mathrm{m})$ : & 14.23 \\
\hline
\end{tabular}

1. based on overall filter area of $1.59 \times 10^{9} \mu \mathrm{m}^{2}$

2. $\mathrm{GMD}=$ geometric mean diameter, $\mathrm{GSD}=$ geometric standard deviation, $\mathrm{R}=$ linear correlation coefficient

Figure 3.73 Module VI, Sample 60A (TSFH16-03) count-based particle size distribution. 


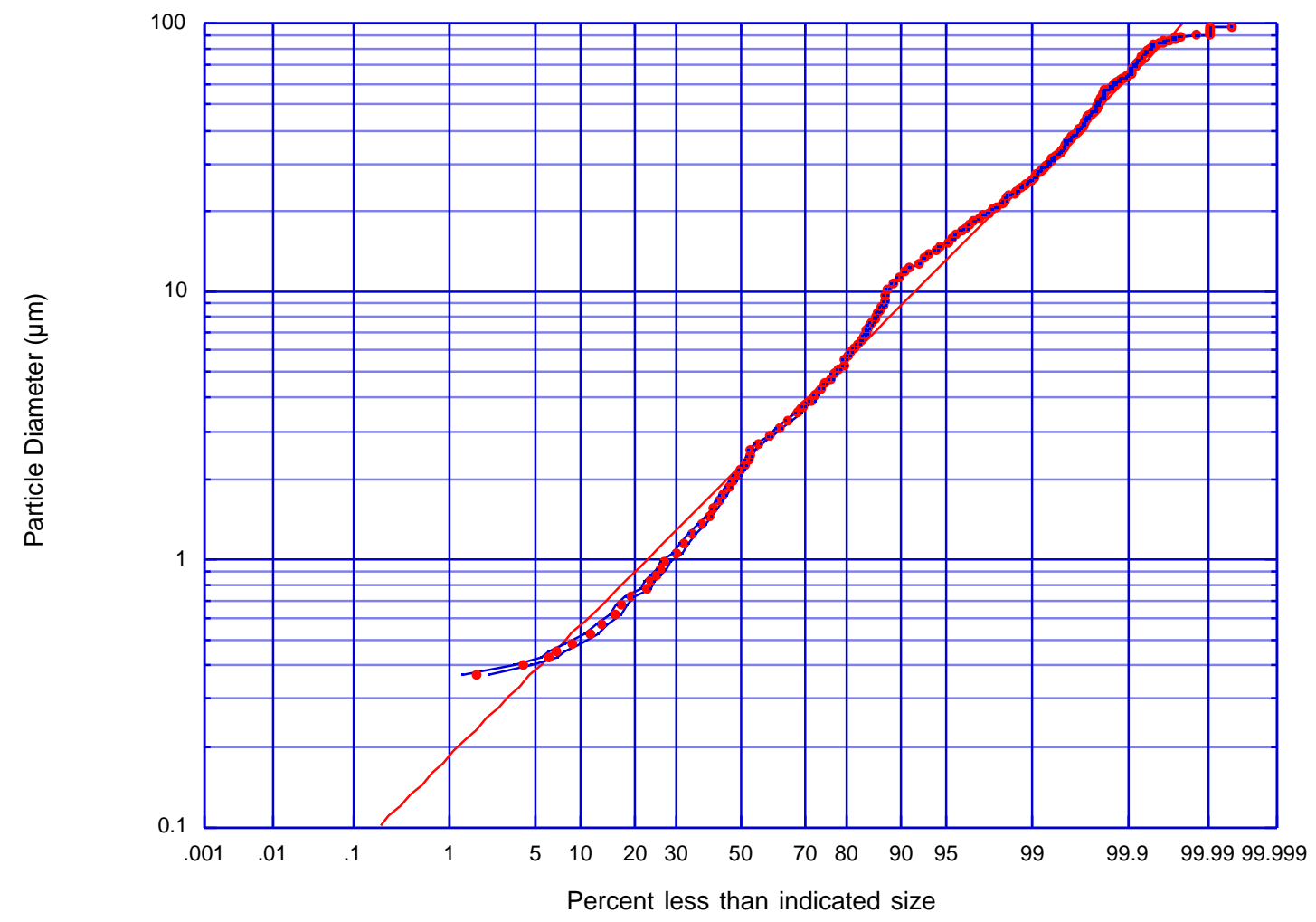

Analysis Summary Table:

\begin{tabular}{|c|c|c|c|c|c|}
\hline \multicolumn{2}{|c|}{$\begin{array}{c}\text { magnification, } \\
\text { number of images }\end{array}$} & \multicolumn{2}{|c|}{ area analyzed $^{1}(\%)$} & counted size range $(\mu \mathrm{m})$ & $\begin{array}{c}\text { total number of } \\
\text { particles (not scaled) }\end{array}$ \\
\hline $50 x$ & 6 & \multicolumn{2}{|r|}{0.894} & $d_{p}>9.43$ & 4826 \\
\hline $100 x$ & 6 & \multicolumn{2}{|r|}{0.224} & $5.18<d_{p}<9.43$ & 2675 \\
\hline $200 x$ & 6 & \multicolumn{2}{|r|}{0.0558} & $2.64<d_{p}<5.18$ & 3119 \\
\hline $500 x$ & 6 & \multicolumn{2}{|r|}{0.00893} & $1.40<\mathrm{d}_{\mathrm{p}}<2.64$ & 1053 \\
\hline 1000x, & 6 & \multicolumn{2}{|r|}{0.00217} & $0.00<\mathrm{d}_{\mathrm{p}}<1.40$ & 599 \\
\hline \multicolumn{2}{|c|}{ Totals: } & \multicolumn{2}{|r|}{$1.18 \%$} & - & 12272 \\
\hline \multicolumn{4}{|c|}{ data moments ${ }^{2}:$} & \multicolumn{2}{|c|}{ log-normal fit moments ${ }^{2}$ : } \\
\hline \multicolumn{3}{|c|}{ GMD $(\mu \mathrm{m}):$} & 2.25 & GMD $(\mu \mathrm{m}):$ & 2.25 \\
\hline \multicolumn{2}{|c|}{ GSD: } & & 3.02 & GSD: & 2.94 \\
\hline \multicolumn{3}{|c|}{ lower $95 \%(\mu \mathrm{m})$ : } & 0.25 & $\mathrm{R}^{2}$ & 0.99347 \\
\hline \multirow{2}{*}{\multicolumn{3}{|c|}{ upper $95 \%(\mu \mathrm{m})$ : }} & 20.52 & lower $95 \%(\mu \mathrm{m})$ : & 0.26 \\
\hline & & & & upper $95 \%(\mu \mathrm{m})$ : & 19.35 \\
\hline
\end{tabular}

1. based on overall filter area of $1.59 \times 10^{9} \mu \mathrm{m}^{2}$

2. $\mathrm{GMD}=$ geometric mean diameter, $\mathrm{GSD}=$ geometric standard deviation, $\mathrm{R}=$ linear correlation coefficient

Figure 3.74 Module VI, Sample 60B (TSFH18-03) count-based particle size distribution. 


\subsubsection{Other Locations Results}

Figure 3.75 through Figure 3.81 display details of particle size distributions for corresponding collection special locations. Table 3.7 gives a summary of the results for special locations.

Table 3.7 Summary of dust collected from specific singular locations.

\begin{tabular}{|c|c|c|c|c|c|c|}
\hline \multirow{2}{*}{$\begin{array}{l}\text { Reference } \\
\text { Location }\end{array}$} & \multirow{2}{*}{ Sample } & \multirow{2}{*}{ Filter ID } & \multirow{2}{*}{$\begin{array}{l}\text { Sampled } \\
\text { Area }\left(\mathrm{cm}^{2}\right)\end{array}$} & \multirow{2}{*}{$\begin{array}{l}\text { Collected } \\
\text { Mass (mg) }\end{array}$} & \multicolumn{2}{|c|}{$\begin{array}{c}\text { Count-based Size } \\
\text { Distribution Parameters }\end{array}$} \\
\hline & & & & & GMD $(\mu \mathrm{m})$ & GSD \\
\hline 71 & 71 & TSFH20-02 & 2,688 & 1.5 & 3.00 & 3.18 \\
\hline 72 & 72 & TSFH20-01 & 2,640 & 2.6 & 3.16 & 3.14 \\
\hline 73 & 73 & TSFH26-01 & 5,520 & 1.1 & 3.68 & 2.89 \\
\hline 74 & 74 & TSFH04-02 & 1,500 & 5.8 & 4.23 & 2.88 \\
\hline 75 & 75 & TSFH01-02 & 1,500 & 6.6 & 3.21 & 2.81 \\
\hline 76 & 76 & TSFH02-02 & 200.0 & $-0.3^{*}$ & 0.98 & 1.93 \\
\hline 77 & 77 & TSFH03-02 & 150.0 & 1.9 & 4.33 & 2.86 \\
\hline
\end{tabular}

*- filter partially broken 


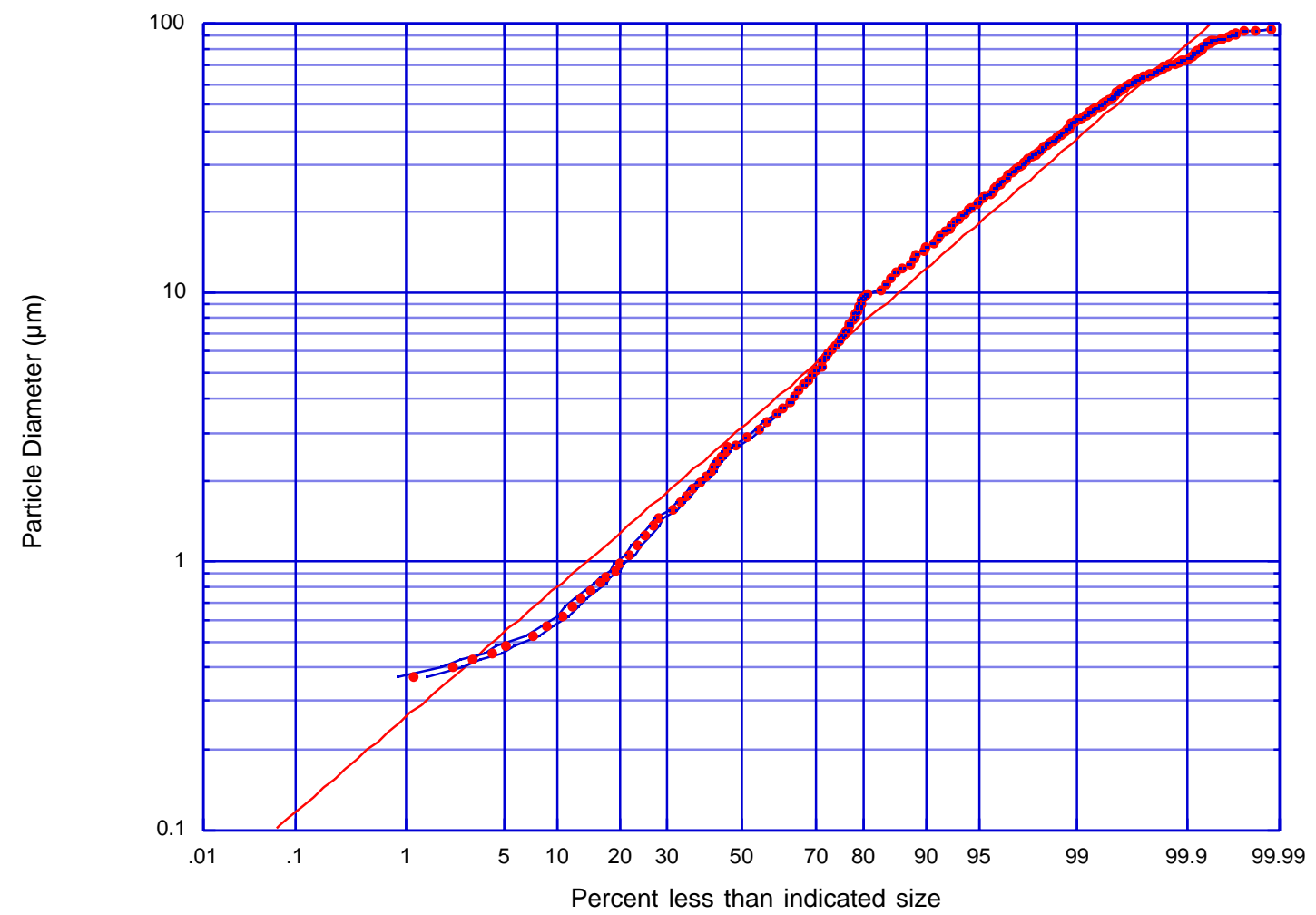

Analysis Summary Table:

\begin{tabular}{|c|c|c|c|c|c|}
\hline \multicolumn{2}{|c|}{$\begin{array}{c}\text { magnification, } \\
\text { number of images }\end{array}$} & \multicolumn{2}{|c|}{ area analyzed $^{1}(\%)$} & counted size range $(\mu \mathrm{m})$ & $\begin{array}{c}\text { total number of } \\
\text { particles (not scaled) }\end{array}$ \\
\hline $50 x$ & 6 & \multicolumn{2}{|r|}{0.894} & $d_{p}>10.40$ & 8830 \\
\hline $100 x$ & 6 & \multicolumn{2}{|r|}{0.224} & $5.22<\mathrm{d}_{\mathrm{p}}<10.40$ & 4362 \\
\hline $200 x$ & 6 & \multicolumn{2}{|r|}{0.0558} & $2.73<\mathrm{d}_{\mathrm{p}}<5.22$ & 4241 \\
\hline $500 x$ & 6 & \multicolumn{2}{|r|}{0.00893} & $1.56<\mathrm{d}_{\mathrm{p}}<2.73$ & 1842 \\
\hline 1000x, & 6 & \multicolumn{2}{|r|}{0.00217} & $0.00<\mathrm{d}_{\mathrm{p}}<1.56$ & 748 \\
\hline \multicolumn{2}{|c|}{ Totals: } & \multicolumn{2}{|r|}{$1.18 \%$} & - & 20023 \\
\hline \multicolumn{4}{|c|}{ data moments ${ }^{2}:$} & \multicolumn{2}{|c|}{ log-normal fit moments ${ }^{2}$ : } \\
\hline \multicolumn{3}{|c|}{ GMD $(\mu \mathrm{m}):$} & 3.00 & GMD $(\mu \mathrm{m}):$ & 3.13 \\
\hline \multicolumn{2}{|c|}{ GSD: } & & 3.18 & GSD: & 2.90 \\
\hline \multicolumn{3}{|c|}{ lower $95 \%(\mu \mathrm{m})$ : } & 0.28 & $\mathrm{R}^{2}$ & 0.98688 \\
\hline \multirow{2}{*}{\multicolumn{3}{|c|}{ upper $95 \%(\mu \mathrm{m})$ : }} & 30.28 & lower $95 \%(\mu \mathrm{m})$ : & 0.37 \\
\hline & & & & upper $95 \%(\mu \mathrm{m})$ : & 26.43 \\
\hline
\end{tabular}

1. based on overall filter area of $1.59 \times 10^{9} \mu \mathrm{m}^{2}$

2. $\mathrm{GMD}=$ geometric mean diameter, $\mathrm{GSD}=$ geometric standard deviation, $\mathrm{R}=$ linear correlation coefficient

Figure 3.75 Sample 71 (TSFH20-02) count-based particle size distribution. 


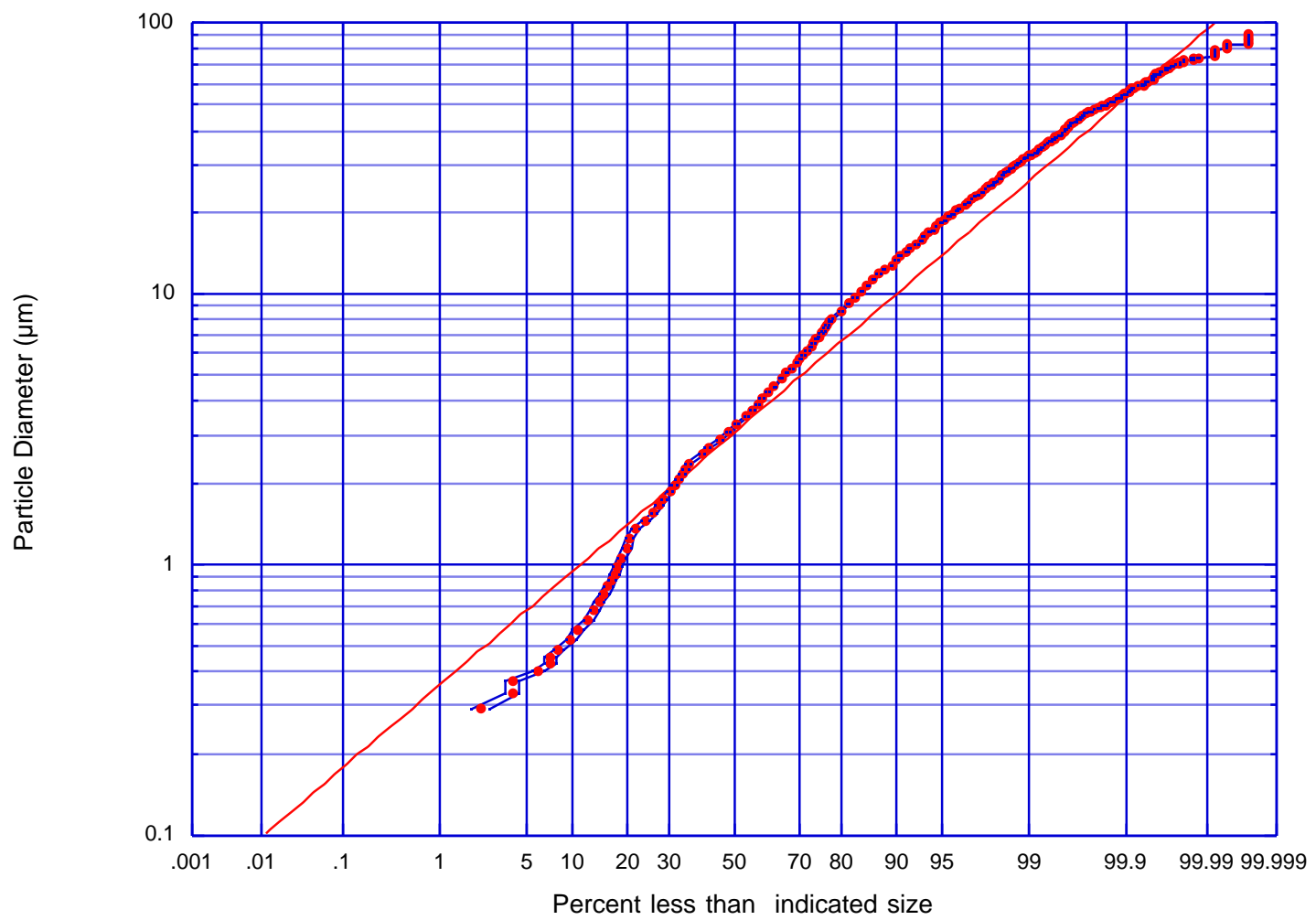

Analysis Summary Table:

\begin{tabular}{|c|c|c|c|c|c|}
\hline \multicolumn{2}{|c|}{$\begin{array}{c}\text { magnification, } \\
\text { number of images }\end{array}$} & \multicolumn{2}{|c|}{ area analyzed $^{1}(\%)$} & counted size range $(\mu \mathrm{m})$ & $\begin{array}{c}\text { total number of } \\
\text { particles (not scaled) }\end{array}$ \\
\hline $50 x$ & 6 & \multicolumn{2}{|r|}{0.894} & $d_{p}>8.25$ & 13377 \\
\hline $100 x$ & 6 & \multicolumn{2}{|r|}{0.224} & $4.39<\mathrm{d}_{\mathrm{p}}<8.25$ & 8853 \\
\hline $200 x$ & 6 & \multicolumn{2}{|r|}{0.0558} & $2.45<\mathrm{d}_{\mathrm{p}}<4.39$ & 5682 \\
\hline $500 x$ & 6 & \multicolumn{2}{|r|}{0.00893} & $1.37<\mathrm{d}_{\mathrm{p}}<2.45$ & 1843 \\
\hline 1000x, & 6 & \multicolumn{2}{|r|}{0.00217} & $0.00<\mathrm{d}_{\mathrm{p}}<1.37$ & 622 \\
\hline \multicolumn{2}{|c|}{ Totals: } & \multicolumn{2}{|r|}{$1.18 \%$} & - & 30377 \\
\hline \multicolumn{4}{|c|}{ data moments ${ }^{2}:$} & \multicolumn{2}{|c|}{ log-normal fit moments ${ }^{2}$ : } \\
\hline \multicolumn{3}{|c|}{ GMD $(\mu \mathrm{m}):$} & 3.16 & GMD $(\mu \mathrm{m}):$ & 3.07 \\
\hline \multicolumn{2}{|c|}{ GSD: } & & 3.14 & GSD: & 2.52 \\
\hline \multicolumn{3}{|c|}{ lower $95 \%(\mu \mathrm{m})$ : } & 0.32 & $\mathrm{R}^{2}$ & 0.97403 \\
\hline \multirow{2}{*}{\multicolumn{3}{|c|}{ upper $95 \%(\mu \mathrm{m})$ : }} & 31.10 & lower $95 \%(\mu \mathrm{m})$ : & 0.48 \\
\hline & & & & upper $95 \%(\mu \mathrm{m})$ : & 19.42 \\
\hline
\end{tabular}

1. based on overall filter area of $1.59 \times 10^{9} \mu \mathrm{m}^{2}$

2. $\mathrm{GMD}=$ geometric mean diameter, $\mathrm{GSD}=$ geometric standard deviation, $\mathrm{R}=$ linear correlation coefficient

Figure 3.76 Sample 72 (TSFH20-01) count-based particle size distribution. 


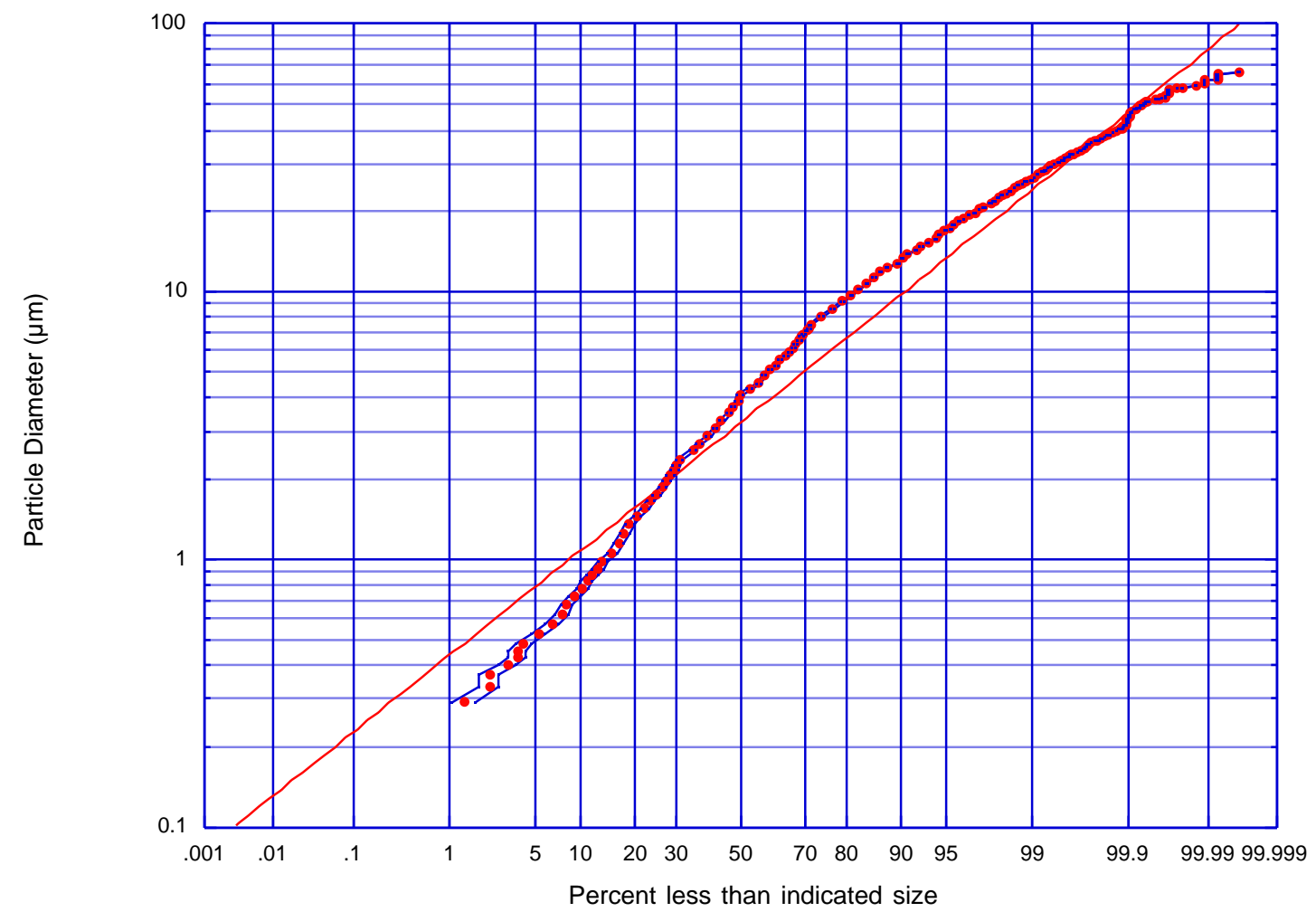

Analysis Summary Table:

\begin{tabular}{|c|c|c|c|c|c|}
\hline \multicolumn{2}{|c|}{$\begin{array}{c}\text { magnification, } \\
\text { number of images }\end{array}$} & \multicolumn{2}{|c|}{ area analyzed $^{1}(\%)$} & counted size range $(\mu \mathrm{m})$ & $\begin{array}{c}\text { total number of } \\
\text { particles (not scaled) }\end{array}$ \\
\hline $50 x$ & 6 & \multicolumn{2}{|r|}{0.894} & $d_{p}>7.75$ & 11424 \\
\hline $100 x$ & 6 & \multicolumn{2}{|r|}{0.224} & $4.25<d_{p}<7.75$ & 6880 \\
\hline $200 x$ & 6 & \multicolumn{2}{|r|}{0.0558} & $2.43<d_{p}<4.25$ & 3450 \\
\hline $500 x$ & 6 & \multicolumn{2}{|r|}{0.00893} & $1.46<\mathrm{d}_{\mathrm{p}}<2.43$ & 1168 \\
\hline 1000x, & 6 & \multicolumn{2}{|r|}{0.00217} & $0.00<\mathrm{d}_{\mathrm{p}}<1.46$ & 413 \\
\hline \multicolumn{2}{|c|}{ Totals: } & \multicolumn{2}{|r|}{$1.18 \%$} & - & 23335 \\
\hline \multicolumn{4}{|c|}{ data moments ${ }^{2}:$} & \multicolumn{2}{|c|}{ log-normal fit moments ${ }^{2}$ : } \\
\hline \multicolumn{3}{|c|}{ GMD $(\mu \mathrm{m}):$} & 3.68 & GMD $(\mu \mathrm{m}):$ & 3.24 \\
\hline \multicolumn{2}{|c|}{ GSD: } & & 2.89 & GSD: & 2.37 \\
\hline \multicolumn{3}{|c|}{ lower $95 \%(\mu \mathrm{m})$ : } & 0.44 & $\mathrm{R}^{2}$ & 0.978 \\
\hline \multirow{2}{*}{\multicolumn{3}{|c|}{ upper $95 \%(\mu \mathrm{m})$ : }} & 30.67 & lower $95 \%(\mu \mathrm{m})$ : & 0.58 \\
\hline & & & & upper $95 \%(\mu \mathrm{m})$ : & 18.18 \\
\hline
\end{tabular}

1. based on overall filter area of $1.59 \times 10^{9} \mu \mathrm{m}^{2}$

2. $\mathrm{GMD}=$ geometric mean diameter, $\mathrm{GSD}=$ geometric standard deviation, $\mathrm{R}=$ linear correlation coefficient

Figure 3.77 Sample 73 (TSFH26-01) count-based particle size distribution. 


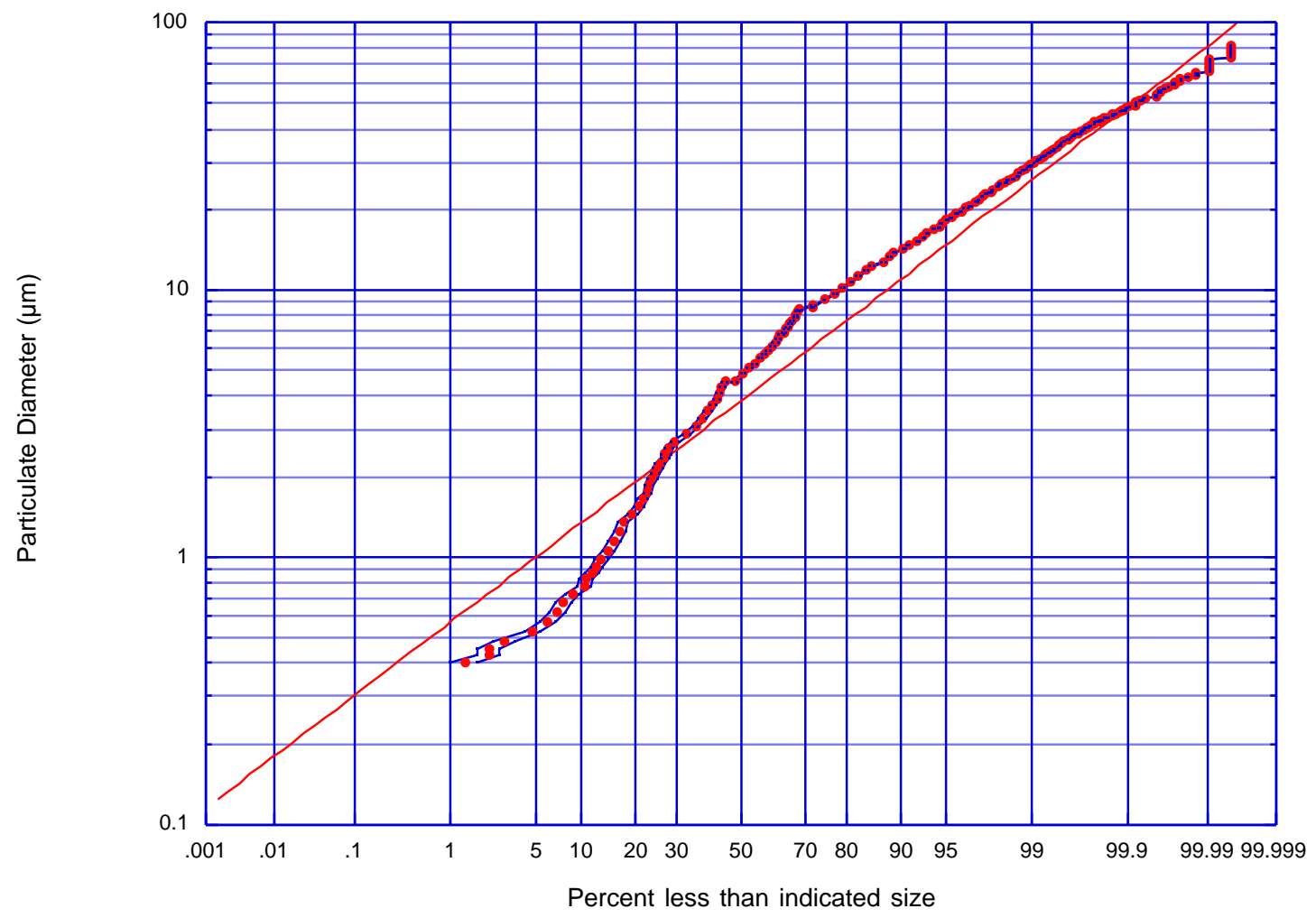

Analysis Summary Table:

\begin{tabular}{|c|c|c|c|c|c|}
\hline \multicolumn{2}{|c|}{$\begin{array}{c}\text { magnification, } \\
\text { number of images }\end{array}$} & \multicolumn{2}{|c|}{ area analyzed $^{1}(\%)$} & counted size range $(\mu \mathrm{m})$ & $\begin{array}{c}\text { total number of } \\
\text { particles (not scaled) }\end{array}$ \\
\hline $50 x$ & 6 & \multicolumn{2}{|r|}{0.894} & $d_{p}>8.83$ & 8579 \\
\hline $100 x$ & 6 & \multicolumn{2}{|r|}{0.224} & $4.74<\mathrm{d}_{\mathrm{p}}<8.83$ & 4567 \\
\hline $200 x$ & 6 & \multicolumn{2}{|r|}{0.0558} & $2.62<d_{p}<4.74$ & 2256 \\
\hline $500 x$ & 6 & \multicolumn{2}{|r|}{0.00893} & $1.42<\mathrm{d}_{\mathrm{p}}<2.62$ & 701 \\
\hline 1000x, & 6 & \multicolumn{2}{|r|}{0.00217} & $0.00<\mathrm{d}_{\mathrm{p}}<1.42$ & 263 \\
\hline \multicolumn{2}{|c|}{ Totals: } & \multicolumn{2}{|r|}{$1.18 \%$} & - & 16366 \\
\hline \multicolumn{4}{|c|}{ data moments ${ }^{2}:$} & \multicolumn{2}{|c|}{ log-normal fit moments ${ }^{2}$ : } \\
\hline \multicolumn{3}{|c|}{ GMD $(\mu \mathrm{m}):$} & 4.23 & GMD $(\mu \mathrm{m}):$ & 3.84 \\
\hline \multicolumn{2}{|c|}{ GSD: } & & 2.88 & GSD: & 2.27 \\
\hline \multicolumn{3}{|c|}{ lower $95 \%(\mu \mathrm{m})$ : } & 0.51 & $\mathrm{R}^{2}$ & 0.97446 \\
\hline \multirow{2}{*}{\multicolumn{3}{|c|}{ upper $95 \%(\mu \mathrm{m})$ : }} & 35.09 & lower $95 \%(\mu \mathrm{m})$ : & 0.74 \\
\hline & & & & upper $95 \%(\mu \mathrm{m})$ : & 19.85 \\
\hline
\end{tabular}

1. based on overall filter area of $1.59 \times 10^{9} \mu \mathrm{m}^{2}$

2. $\mathrm{GMD}=$ geometric mean diameter, $\mathrm{GSD}=$ geometric standard deviation, $\mathrm{R}=$ linear correlation coefficient

Figure 3.78 Sample 74 (TSFH04-02) count-based particle size distribution. 


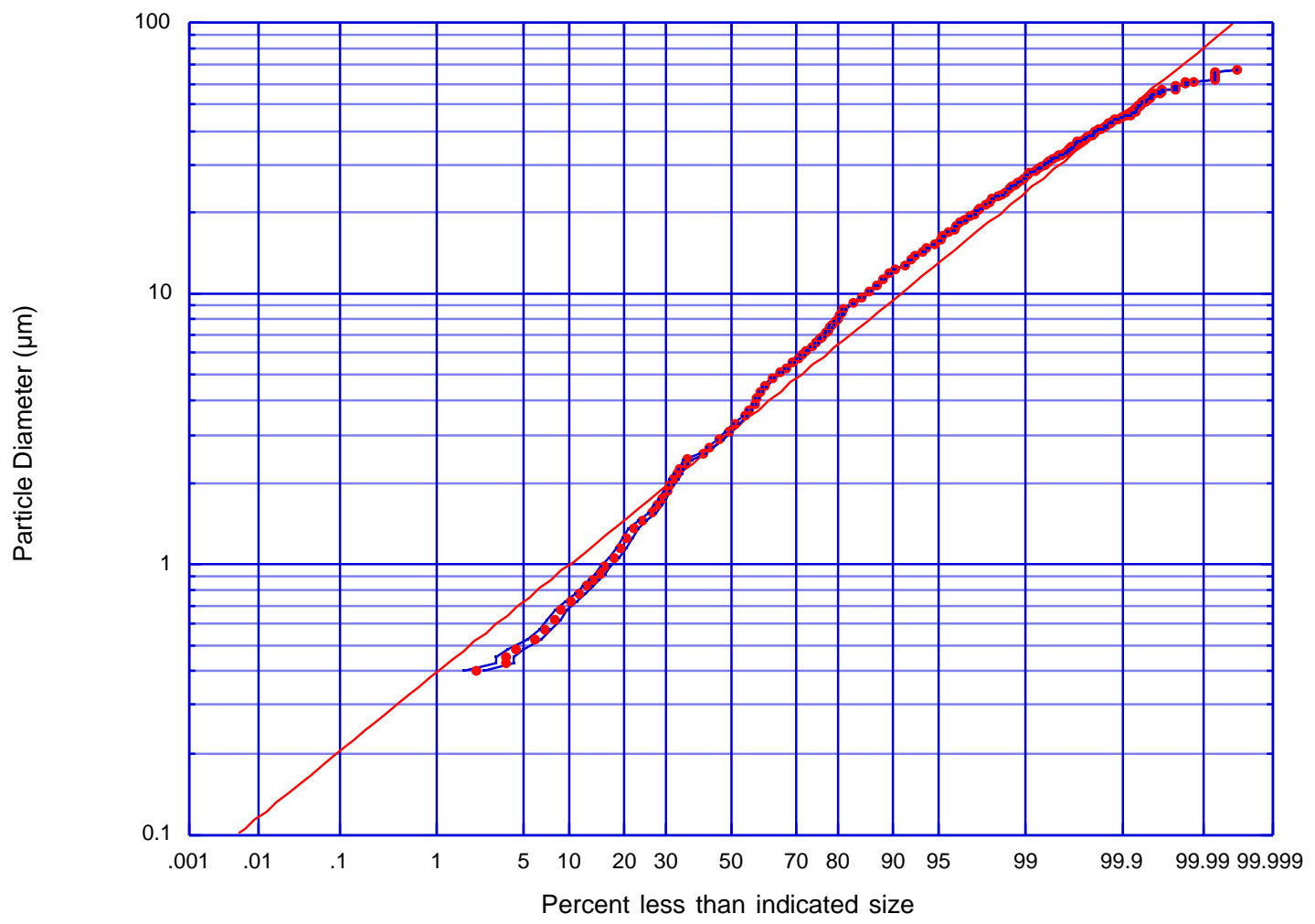

Analysis Summary Table:

\begin{tabular}{|c|c|c|c|c|c|}
\hline \multicolumn{2}{|c|}{$\begin{array}{c}\text { magnification, } \\
\text { number of images }\end{array}$} & \multicolumn{2}{|c|}{ area analyzed $^{1}(\%)$} & counted size range $(\mu \mathrm{m})$ & $\begin{array}{c}\text { total number of } \\
\text { particles (not scaled) }\end{array}$ \\
\hline $50 x$ & 6 & \multicolumn{2}{|r|}{0.894} & $d_{p}>8.83$ & 8146 \\
\hline $100 x$ & 6 & \multicolumn{2}{|r|}{0.224} & $4.70<\mathrm{d}_{\mathrm{p}}<8.83$ & 6488 \\
\hline $200 x$ & 6 & \multicolumn{2}{|r|}{0.0558} & $2.52<d_{p}<4.70$ & 3624 \\
\hline $500 x$ & 6 & \multicolumn{2}{|r|}{0.00893} & $1.40<\mathrm{d}_{\mathrm{p}}<2.52$ & 1189 \\
\hline 1000x, & 6 & \multicolumn{2}{|r|}{0.00217} & $0.00<\mathrm{d}_{\mathrm{p}}<1.40$ & 460 \\
\hline \multicolumn{2}{|c|}{ Totals: } & \multicolumn{2}{|r|}{$1.18 \%$} & - & 19907 \\
\hline \multicolumn{4}{|c|}{ data moments ${ }^{2}:$} & \multicolumn{2}{|c|}{ log-normal fit moments ${ }^{2}$ : } \\
\hline \multicolumn{3}{|c|}{ GMD $(\mu \mathrm{m}):$} & 3.21 & GMD $(\mu \mathrm{m}):$ & 3.07 \\
\hline \multicolumn{2}{|c|}{ GSD: } & & 2.81 & GSD: & 2.41 \\
\hline \multicolumn{3}{|c|}{ lower $95 \%(\mu \mathrm{m})$ : } & 0.41 & $\mathrm{R}^{2}$ & 0.98351 \\
\hline \multirow{2}{*}{\multicolumn{3}{|c|}{ upper $95 \%(\mu \mathrm{m})$ : }} & 25.41 & lower $95 \%(\mu \mathrm{m})$ : & 0.53 \\
\hline & & & & upper $95 \%(\mu \mathrm{m})$ : & 17.86 \\
\hline
\end{tabular}

1. based on overall filter area of $1.59 \times 10^{9} \mu \mathrm{m}^{2}$

2. $\mathrm{GMD}=$ geometric mean diameter, $\mathrm{GSD}=$ geometric standard deviation, $\mathrm{R}=$ linear correlation coefficient

Figure 3.79 Sample 75 (TSFH01-02) count-based particle size distribution. 


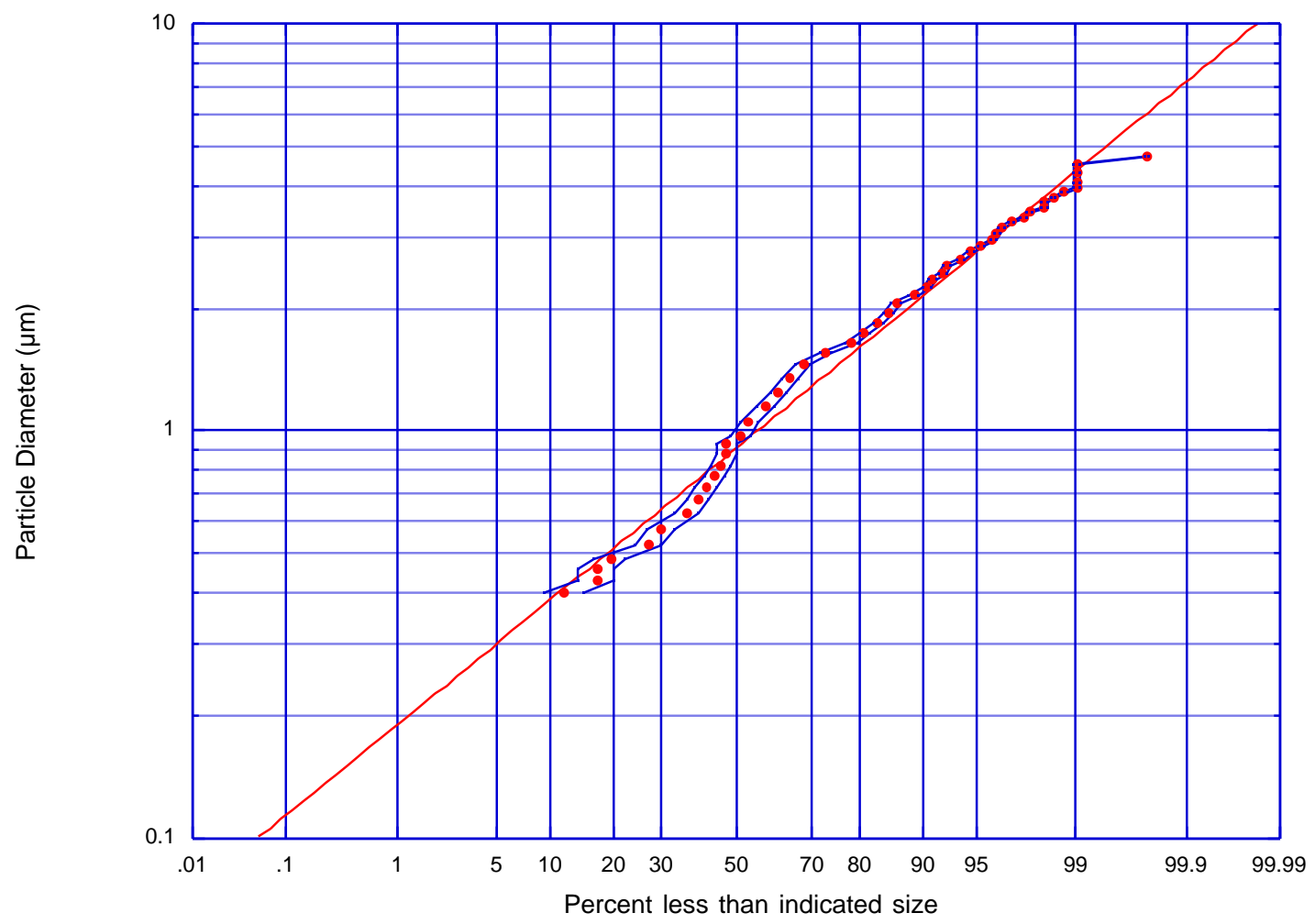

Analysis Summary Table:

\begin{tabular}{|c|c|c|c|c|c|}
\hline \multicolumn{2}{|c|}{$\begin{array}{c}\text { magnification, } \\
\text { number of images }\end{array}$} & \multicolumn{2}{|c|}{ area analyzed $^{1}(\%)$} & counted size range $(\mu \mathrm{m})$ & $\begin{array}{c}\text { total number of } \\
\text { particles (not scaled) }\end{array}$ \\
\hline $50 x$ & 6 & \multicolumn{2}{|r|}{0.0} & NA & NA \\
\hline $100 x$ & 6 & \multicolumn{2}{|r|}{0.0} & NA & NA \\
\hline $200 x$ & 6 & \multicolumn{2}{|r|}{0.0} & NA & NA \\
\hline $500 x$ & 6 & \multicolumn{2}{|r|}{0.00893} & $\mathrm{~d}_{\mathrm{p}}>1.16$ & 173 \\
\hline 1000x, & 6 & \multicolumn{2}{|r|}{0.00217} & $0.00<\mathrm{d}_{\mathrm{p}}<1.16$ & 268 \\
\hline \multicolumn{2}{|c|}{ Totals: } & \multicolumn{2}{|r|}{$0.01 \%$} & - & 441 \\
\hline \multicolumn{4}{|c|}{ data moments ${ }^{2}:$} & \multicolumn{2}{|c|}{ log-normal fit moments ${ }^{2}$ : } \\
\hline \multicolumn{3}{|c|}{ GMD $(\mu \mathrm{m}):$} & 0.98 & GMD $(\mu \mathrm{m}):$ & 0.91 \\
\hline \multicolumn{2}{|c|}{ GSD: } & & 1.93 & GSD: & 1.96 \\
\hline \multicolumn{3}{|c|}{ lower $95 \%(\mu \mathrm{m})$ : } & 0.26 & $\mathrm{R}^{2}:$ & 0.98738 \\
\hline \multicolumn{3}{|c|}{ upper $95 \%(\mu \mathrm{m})$ : } & 3.63 & lower $95 \%(\mu \mathrm{m})$ : & 0.24 \\
\hline & & & & upper $95 \%(\mu \mathrm{m})$ : & 3.49 \\
\hline
\end{tabular}

1. based on overall filter area of $1.59 \times 10^{9} \mu \mathrm{m}^{2}$

2. $\mathrm{GMD}=$ geometric mean diameter, $\mathrm{GSD}=$ geometric standard deviation, $\mathrm{R}=$ linear correlation coefficient

Figure 3.80 Sample 76 (TSFH02-02) count-based particle size distribution. 


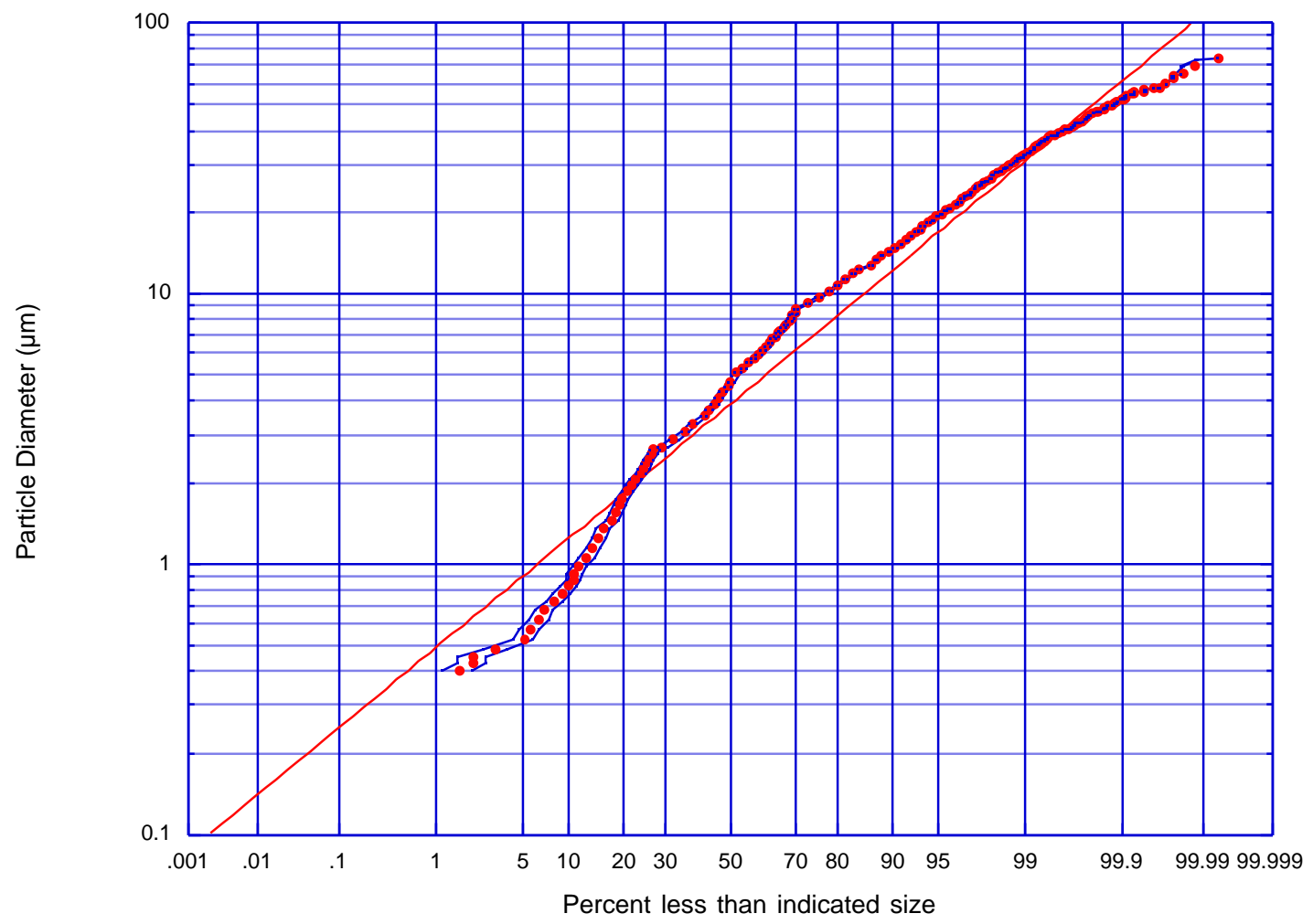

Analysis Summary Table:

\begin{tabular}{|c|c|c|c|c|c|}
\hline \multicolumn{2}{|c|}{$\begin{array}{c}\text { magnification, } \\
\text { number of images }\end{array}$} & \multicolumn{2}{|c|}{ area analyzed $^{1}(\%)$} & counted size range $(\mu \mathrm{m})$ & $\begin{array}{c}\text { total number of } \\
\text { particles (not scaled) }\end{array}$ \\
\hline $50 x$ & 6 & \multicolumn{2}{|r|}{0.894} & $d_{p}>8.98$ & 7054 \\
\hline $100 x$ & 6 & \multicolumn{2}{|r|}{0.224} & $4.95<\mathrm{d}_{\mathrm{p}}<8.98$ & 3837 \\
\hline $200 x$ & 6 & \multicolumn{2}{|r|}{0.0558} & $2.74<\mathrm{d}_{\mathrm{p}}<4.95$ & 2099 \\
\hline $500 x$ & 6 & \multicolumn{2}{|r|}{0.00893} & $1.62<d_{p}<2.74$ & 600 \\
\hline 1000x, & 6 & \multicolumn{2}{|r|}{0.00217} & $0.00<\mathrm{d}_{\mathrm{p}}<1.62$ & 244 \\
\hline \multicolumn{2}{|c|}{ Totals: } & \multicolumn{2}{|r|}{$1.18 \%$} & - & 13834 \\
\hline \multicolumn{4}{|c|}{ data moments ${ }^{2}:$} & \multicolumn{2}{|c|}{ log-normal fit moments ${ }^{2}$ : } \\
\hline \multicolumn{3}{|c|}{ GMD $(\mu \mathrm{m}):$} & 4.33 & GMD $(\mu \mathrm{m}):$ & 3.90 \\
\hline \multicolumn{2}{|c|}{ GSD: } & & 2.86 & GSD: & 2.44 \\
\hline \multicolumn{3}{|c|}{ lower $95 \%(\mu \mathrm{m})$ : } & 0.53 & $\mathrm{R}^{2}$ & 0.9783 \\
\hline \multirow{2}{*}{\multicolumn{3}{|c|}{ upper $95 \%(\mu \mathrm{m})$ : }} & 35.35 & lower $95 \%(\mu \mathrm{m})$ : & 0.66 \\
\hline & & & & upper $95 \%(\mu \mathrm{m})$ : & 23.23 \\
\hline
\end{tabular}

1. based on overall filter area of $1.59 \times 10^{9} \mu \mathrm{m}^{2}$

2. $\mathrm{GMD}=$ geometric mean diameter, $\mathrm{GSD}=$ geometric standard deviation, $\mathrm{R}=$ linear correlation coefficient

Figure 3.81 Sample 77 (TSFH03-02) count-based particle size distribution. 


\subsection{Size Analysis with the Coulter LS130 Particle Analysis System}

\subsubsection{Technique}

The Coulter LS130 Particle Size Analysis System is an instrument that uses the diffraction of laser light by particles suspended in a transparent liquid medium. Mie scattering theory is used to deconvolve a time-averaged measurement (obtained via photodiode detectors displaced in angle about the beam centerline) of the diffraction pattern generated by the particulate population exposed to the collimated laser beam. Resulting from the measurement and deconvolution algorithm is the volume-based size distribution of the particulate in the measurement cell. Such a measurement is useful because it provides information regarding the mass distribution of the particulate if the sample is composed of particles with equal-density. When compared to the count-based distribution (obtained by the method described in Section 3.2) of the same particulate, the measured volume-based distribution may be used to indicate shape of the average particle, even when all particles are not equally dense. The Coulter LS130 has a manufacturerspecified size measurement range of $0.4-1000 \mu \mathrm{m}$.

Size analysis with this instrument requires the particulate sample (often milligrams in quantity) to be added to the diluent as a dry powder. Only samples collected in the lower regions of the Tore Supra vessel provided sufficient quantity for analysis. An additional requirement involves efficient removal of the smallest particulate $(>\sim 5 \mu \mathrm{m})$ from the filter substrate. Difficulties were encountered in fulfilling this requirement because the small particles strongly adhere to the filter substrate. Repeated washing of the filters with pure water and/or ethanol did remove some of the smaller particulate, as indicated upon microscopy inspection following the wash. Because of these difficulties, only one dust sample from Tore Supra was successfully analyzed with the Coulter LS130. It is recommended that in the future a significant effort is made to collect vastly greater quantities of dust (provided they exist) with an alternative collection technique, such as mechanically removing the particulate, i.e. sweeping with a fine brush into a collection bin. Note however that all collection techniques display size-range bias in collection efficiency.

\subsubsection{Results}

Particulate collected from the Tore Supra vessel bottom in Module V has been successfully analyzed with the Coulter LS130 particle size analysis system. Shown in Figure 3.83 is the resulting volume-based size distribution of particulate removed from the filter substrate (TSFH23-02) via repeated washing in pure water followed by ethanol. The analysis medium was also ethanol. Results from two rinsing trials are shown on the figure. Trial 1 was performed and two independent measurements were taken with the LS130. Samples for each measurement were obtained from the same particle-ethanol mixture from Trial 1. Effluent from the next rinsing trial (Trial 2) was then added to that of Trial 1, and three separate measurements were obtained with the LS130. As indicated by the larger differential volume (y-axis in the figure) values at lower particle sizes $(\sim 0.5-2 \mu \mathrm{m})$ from Trial 2 and nearly equivalent values at larger sizes $(\sim 10-100 \mu \mathrm{m})$ from both trials, several additional small particles were removed during the second rinse cycle. Hence the distributions measured from the Trial 2 measurements are believed to most likely represent the volume-based distribution of this Tore Supra dust sample. 


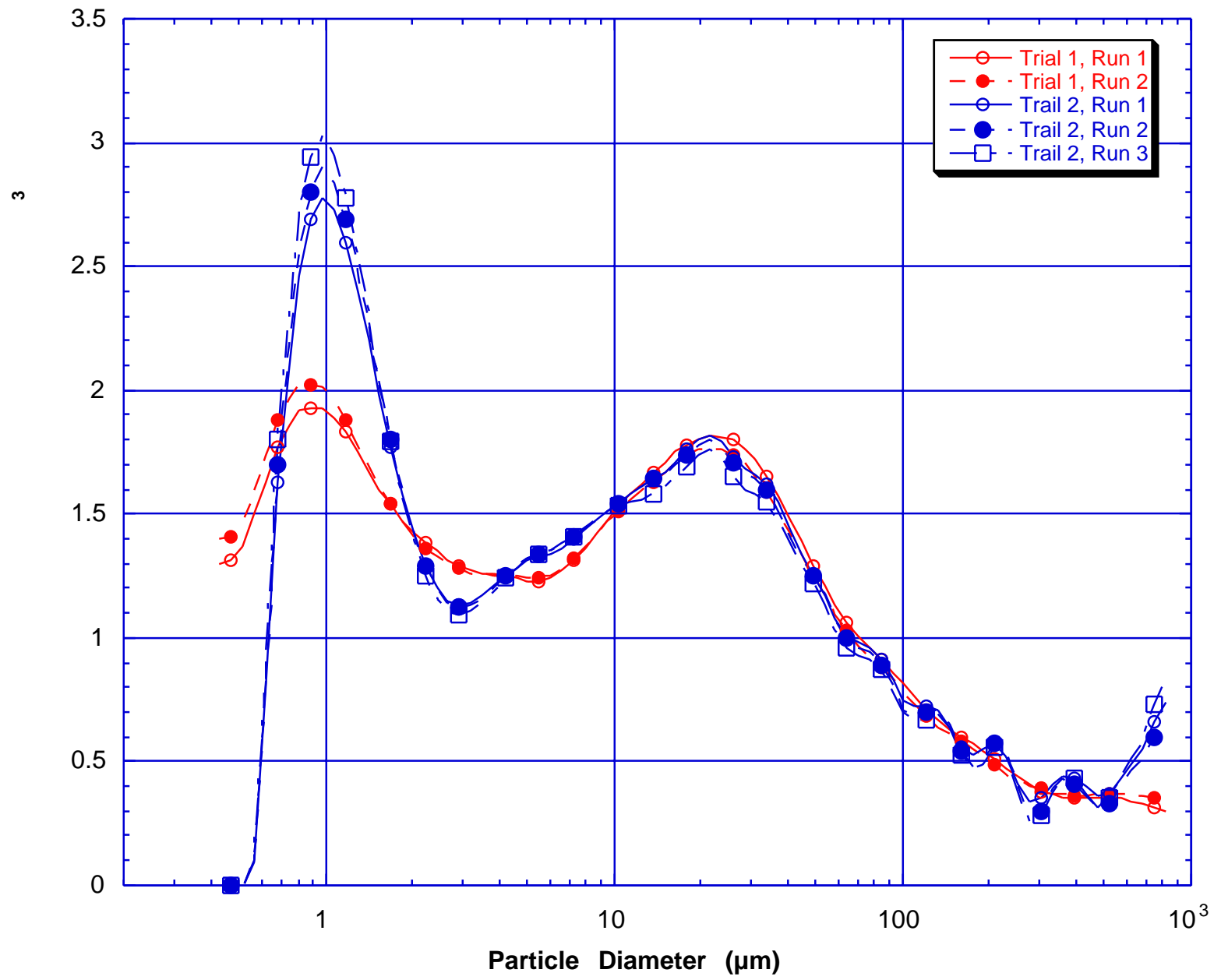

Figure 3.82 Volume-based size distribution of Module V, Sample 50A (TSFH22-03).

An inference may be drawn from the volume-based size distribution shown in Figure 3.82. Two distinct maxima are observed, one centered about $1 \mu \mathrm{m}$ and the other centered about $23 \mu \mathrm{m}$. A large population of particulate exists in the 1-2 $\mu \mathrm{m}$ range, and they are roughly spherical in shape since this measurement agrees with the count-based measurement (GMD of $1.85 \mu \mathrm{m}$ ) given in Figure 3.61 of Section 3.2. Another population with a much larger variance appears in this dust sample. This subset does not correspond to spherical particulate and agrees with the observed bi-modality found in Figure 3.61. The inference regarding the existence of varying particle in this dust sample is not unexpected since many particle shapes were found, as discussed in Section 3.1. 


\section{Specific Surface Area Analysis}

The total effective surface area of dust in a fusion device is an important factor in understanding the consequences of postulated accidents considered in safety analyses. For example, reactive dust may yield explosive concentrations of $\mathrm{H}_{2}$ when exposed to steam or moist air in loss of vacuum ingress accidents. Measurements of the specific surface area $\left(\mathrm{m}^{2}\right.$ per gram) of dust from operating fusion experiments can provide fundamental data for such safety analyses.

\subsection{Technique}

Measurement of specific surface area of dust collected from Tore Supra was performed with the Micromeritics Model ASAP 2010 accelerated surface area and porosity system. The system is designed to use a static volumetric method for determining the volume of gas adsorbed on a sample at constant temperature $(77 \mathrm{~K})$. Measurements of high accuracy are obtained for the adsorptive gas pressure and required volume that fills a control manifold, the sample container, and that adsorbed on the sample surface. Pressures used for this measurement are varied from 0 (vacuum) to approximately $50 \%$ of the vapor saturation pressure at the analysis temperature. The molar quantity of adsorbate is computed from the measured pressure $P$ and adsorbed volume $V$ (normalized to sample mass) by utilizing the BET multilayer physisorption isotherm ${ }^{6}$ :

$$
\frac{1}{V\left(P_{o} / P-1\right)}=\frac{(c-1) P}{c V_{m} P_{o}}+\frac{1}{c V_{m}}
$$

where $P_{o}$ is the vapor pressure at the analysis temperature, $V_{m}$ is the monlayer volume of adsorbate per unit sample mass, and $c$ is a constant. As $V, P$, and $P_{o}$ are known, a linear fit of this equation in $P / P_{o}$ will yield values of $c$ and $V_{m}$. The sample's specific surface area (SSA, $\left.\mathrm{m}^{2} / \mathrm{g}\right)$ at STP $(273 \mathrm{~K}$ and $1.0 \mathrm{~atm})$ is then found by:

$$
S S A=\frac{V_{m} m_{s} N_{A} \sigma_{o}}{0.0224}
$$

where $m_{s}$ is the sample mass in grams, $N_{A}$ is Avagadro's number, and $\sigma_{o}$ is the molecular diameter $\left(\mathrm{m}^{2}\right)$ of the adsorbate (e.g. $0.21 \mathrm{E}-19 \mathrm{~m}^{2}$ for $\mathrm{Kr}$ ). The constant in the denominator of Equation 2 is the volume in $\mathrm{m}^{3}$ of 1 mole of an ideal gas at STP $(\mathrm{T}=300 \mathrm{~K}, \mathrm{P}=101325 \mathrm{~Pa})$. Due to the small quantities of sample mass available for testing, special procedures have been developed $^{7}$ to enhance the sensitivity of the ASAP 2010. These procedures include the use of $\mathrm{Kr}$ as the analysis gas and a novel background volume subtraction technique.

\subsection{Results}

The specific surface area measurements were performed on dust samples taken from the following locations:

i. bottom Location 12 of Module III (TSFH21-02, Sample 30B)

ii. $\quad$ outboard Location 2 of Module IV (TSFH16-02, Sample 32) 
iii. bottom Location 11 of Module IV (TSFH15-02, Sample 40B)

iv. bottom Location 12 of Module IV (TSFH19-02, Sample 40A)

These locations were selected because the total sample mass collected from each position was sufficiently large to provide sufficient surface area for measurement. Particulate composition of the samples from these locations included carbon and metal with unknown weight proportions, thereby rendering unknown the components' relative contribution to surface area.

Figure 4.1 through Figure 4.4 show the measured values of (a) adsorbed volume and (b) adsorption isotherms for each sample. Two runs in the ASAP2100 were made for each sample, with a bakeout period between runs. A linear fit of the entire data range on some of the isotherm plots (e.g. Figure 4.2(b) and Figure 4.4(b)) yielded poor linear correlation coefficients $\left(R^{2}\right)$, such that another fit was performed over a reduced range with an improved linear trend. Fits that gives $R^{2}>0.99$ where used to determine averaged sample $S S A$ results. Table 4.1 summarizes the measurements and the resulting $S S A$ values for the dust samples.

Table 4.1 Summary of results from BET SSA measurements.

\begin{tabular}{|l|l|c|c|}
\hline \multicolumn{3}{|c|}{ Sample 1. } \\
\hline \multicolumn{3}{|c|}{ Location 12 of Module III (TSFH21-02, Sample 30B) } \\
\hline \multicolumn{2}{|c|}{ Fitted Range } & SSA $\mathrm{m}^{2} / \mathrm{g}$ & $R^{2}$ \\
\hline \hline \multirow{2}{*}{ Run 1: } & Full & $1.2009 \pm 0.0215$ & 0.99633 \\
\cline { 2 - 4 } & Partial: & $1.1307 \pm 0.0104$ & 0.99950 \\
\hline \multirow{2}{*}{ Run 2: } & Full: & $1.3075 \pm 0.0134$ & 0.99881 \\
\cline { 2 - 4 } & Partial: & $1.2806 \pm 0.0073$ & 0.99980 \\
\hline \multicolumn{3}{|c|}{ Sample average SSA: $1.230 \pm 0.013 \mathrm{~m}^{2} / \mathrm{g}$} \\
\hline
\end{tabular}

\begin{tabular}{|l|l|c|c|}
\hline \multicolumn{4}{|c|}{ Sample 2. } \\
\hline \multicolumn{3}{|c|}{ Location 2 of Module IV (TSFH16-02, Sample 32) } \\
\hline \multicolumn{2}{|c|}{ Fitted Range } & \multicolumn{1}{|c|}{$S S A, \mathrm{~m}^{2} / \mathrm{g}$} & $R^{2}$ \\
\hline \hline \multirow{2}{*}{ Run 1: } & Full & $2.2780 \pm 0.9969$ & 0.29159 \\
\cline { 2 - 4 } & Partial: & $1.2109 \pm 0.1313$ & 0.99731 \\
\hline \multirow{2}{*}{ Run 2: } & Full: & $2.0203 \pm 0.6310$ & 0.46248 \\
\cline { 2 - 4 } & Partial: & $1.3025 \pm 0.0337$ & 0.99777 \\
\hline \multirow{3}{|l|}{ Sample average $S S A: 1.257 \pm 0.083 \mathrm{~m}^{2} / \mathrm{g}$} \\
\hline
\end{tabular}

\begin{tabular}{|l|l|c|c|}
\hline \multicolumn{3}{|c|}{ Sample 3. } \\
\hline \multicolumn{2}{|c|}{ Location 11 of Module IV (TSFH15-02, Sample 40B) } \\
\hline \multirow{2}{*}{ Fitted Range } & SSA $\mathrm{m}^{2} / \mathrm{g}$ & $R^{2}$ \\
\hline \hline \multirow{2}{*}{ Run 1: } & Full & NA & NA \\
\cline { 2 - 4 } & Partial: & $1.1990 \pm 0.0081$ & 0.99976 \\
\hline \multirow{2}{*}{ Run 2: } & Full: & $1.5945 \pm 0.0680$ & 0.99980 \\
\cline { 2 - 4 } & Partial: & $1.5987 \pm 0.0038$ & 0.99996 \\
\hline \multicolumn{3}{|l|}{ Sample average SSA: $1.464 \pm 0.026 \mathrm{~m}^{2} / \mathrm{g}$} \\
\hline
\end{tabular}

\begin{tabular}{|l|l|c|c|}
\hline \multicolumn{3}{|c|}{ Sample 4. } \\
\hline \multicolumn{3}{|c|}{ Location 12 of Module IV (TSFH19-02, Sample 40A) } \\
\hline \multicolumn{2}{|c|}{ Fitted Range } & \multicolumn{1}{|c|}{$S S A, \mathrm{~m}^{2} / \mathrm{g}$} & $R^{2}$ \\
\hline \hline \multirow{2}{*}{ Run 1: } & Full & $0.4473 \pm 0.0315$ & 0.94629 \\
\cline { 2 - 4 } & Partial: & $0.3923 \pm 0.0950$ & 0.99781 \\
\hline \multirow{2}{*}{ Run 2: } & Full: & $0.2683 \pm 0.0221$ & 0.93495 \\
\cline { 2 - 4 } & Partial: & $0.2400 \pm 0.0084$ & 0.99720 \\
\hline \multicolumn{3}{|l|}{ Sample average SSA: $0.316 \pm 0.052 \mathrm{~m}^{2} / \mathrm{g}$} \\
\hline
\end{tabular}

The value obtained for the outboard sample (ii) was comparable to the values obtained for vessel bottom samples. The value for the bottom sample (iv), however, is lower than the measurements from other bottom locations, possibly due to a greater contribution of metals. Continued investigation is underway to determine the degree of influence of dust composition on specific surface area. The specific surface area measurements for dust in Tore Supra are consistent in magnitude with the average values obtained from other fusion experiments ${ }^{7}: 2.44$ $\mathrm{m}^{2} / \mathrm{g}$ in DIII-D, $0.77 \mathrm{~m}^{2} / \mathrm{g}$ in Alcator C-Mod, and $0.82 \mathrm{~m}^{2} / \mathrm{g}$ in TFTR (vacuum filter analysis with $\mathrm{Kr}$ gas). 


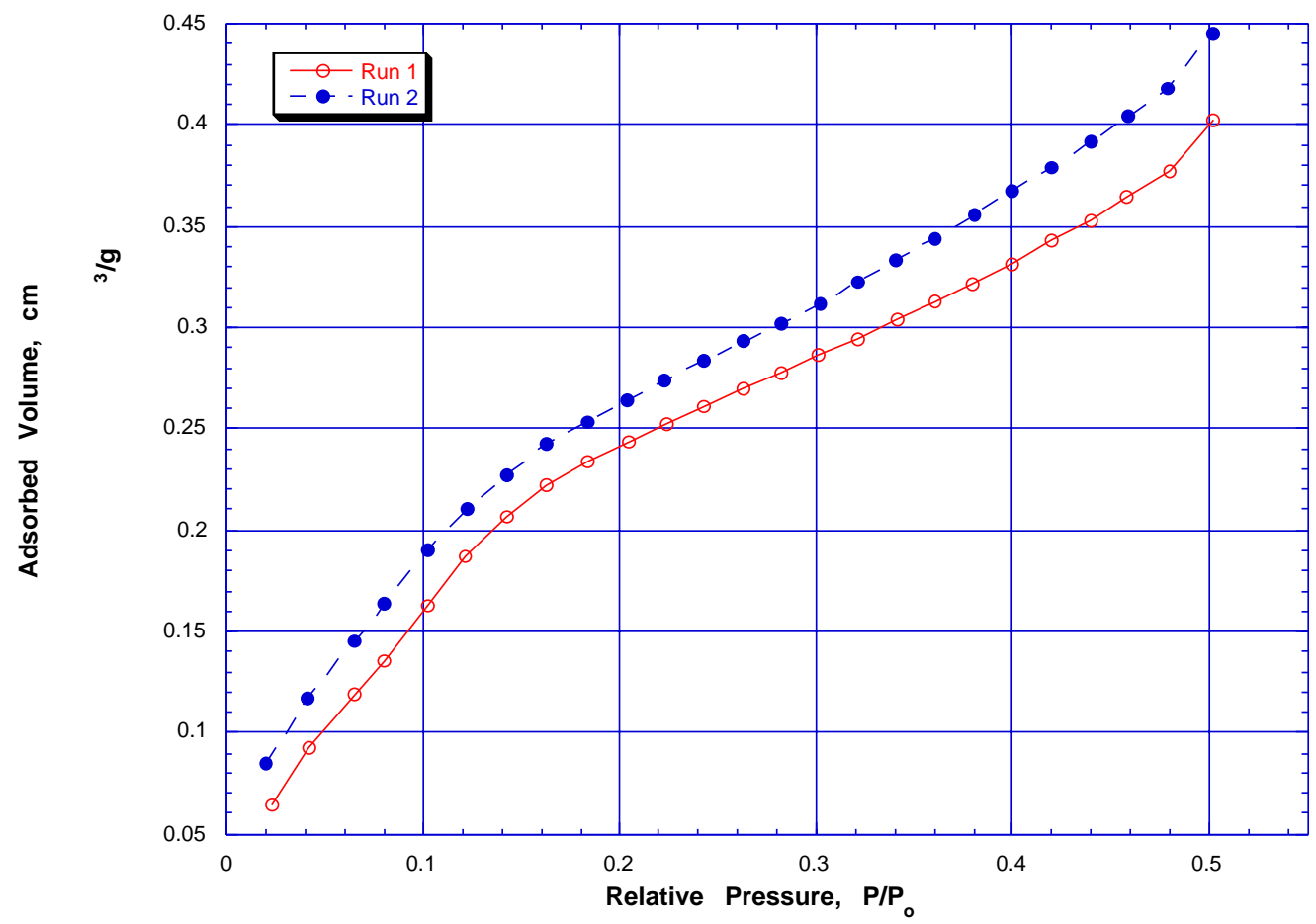

(a) Adsorbed Volume

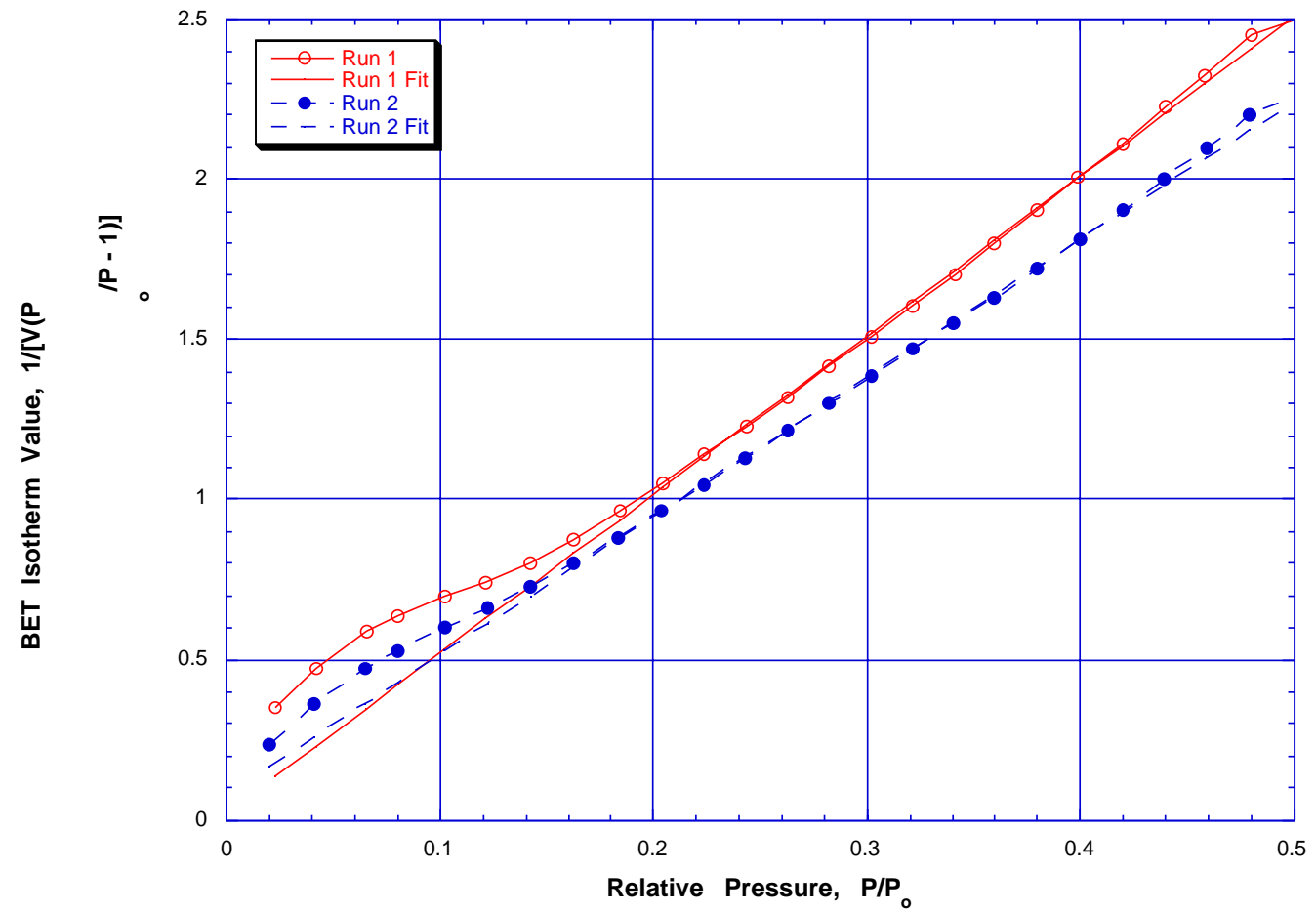

(b) Isotherm

Figure 4.1 Adsorbed Volume (a) and adsorption isotherm (b) for (iv) TSFH21-02, Sample 30B. 


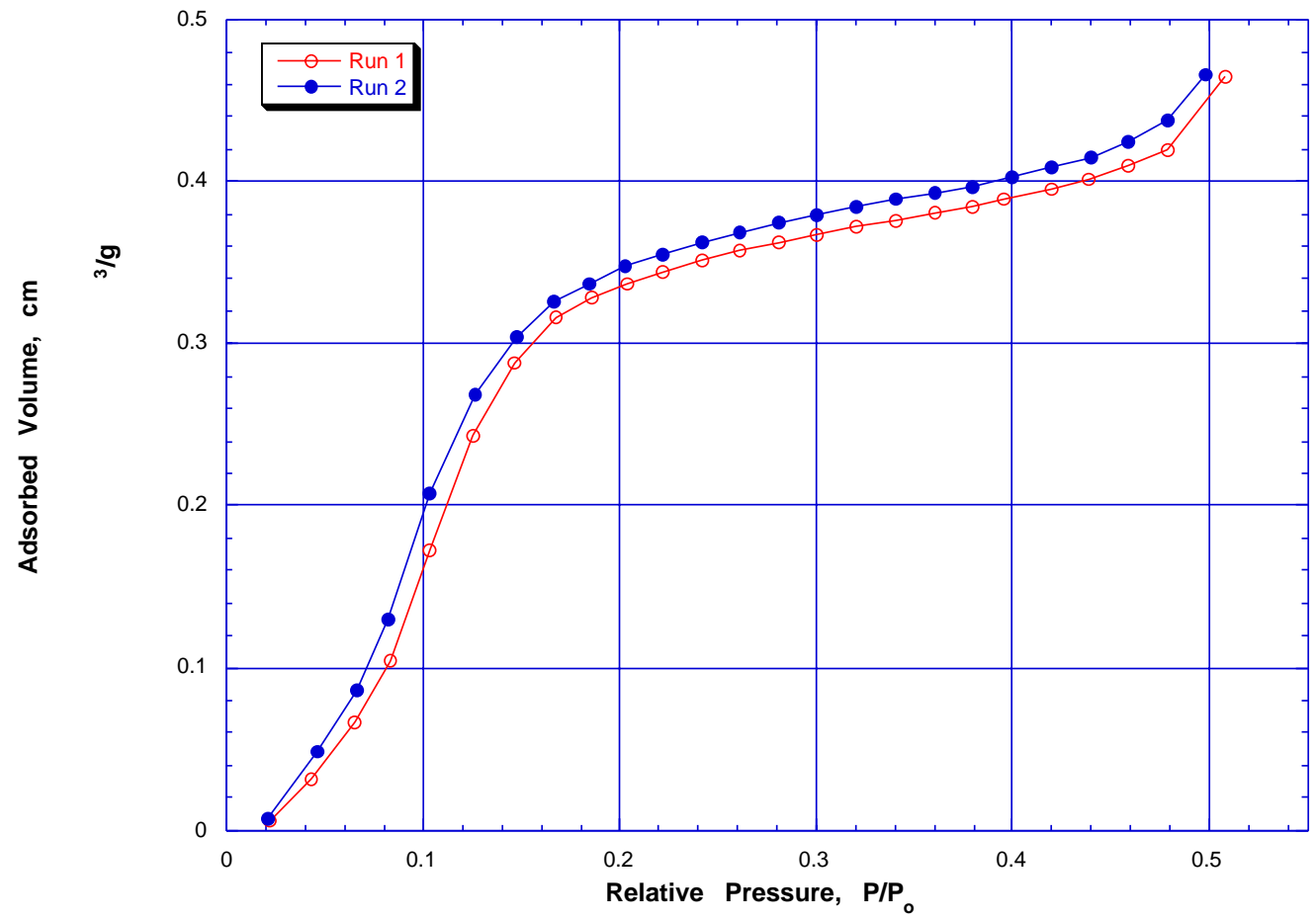

(a) Adsorbed Volume

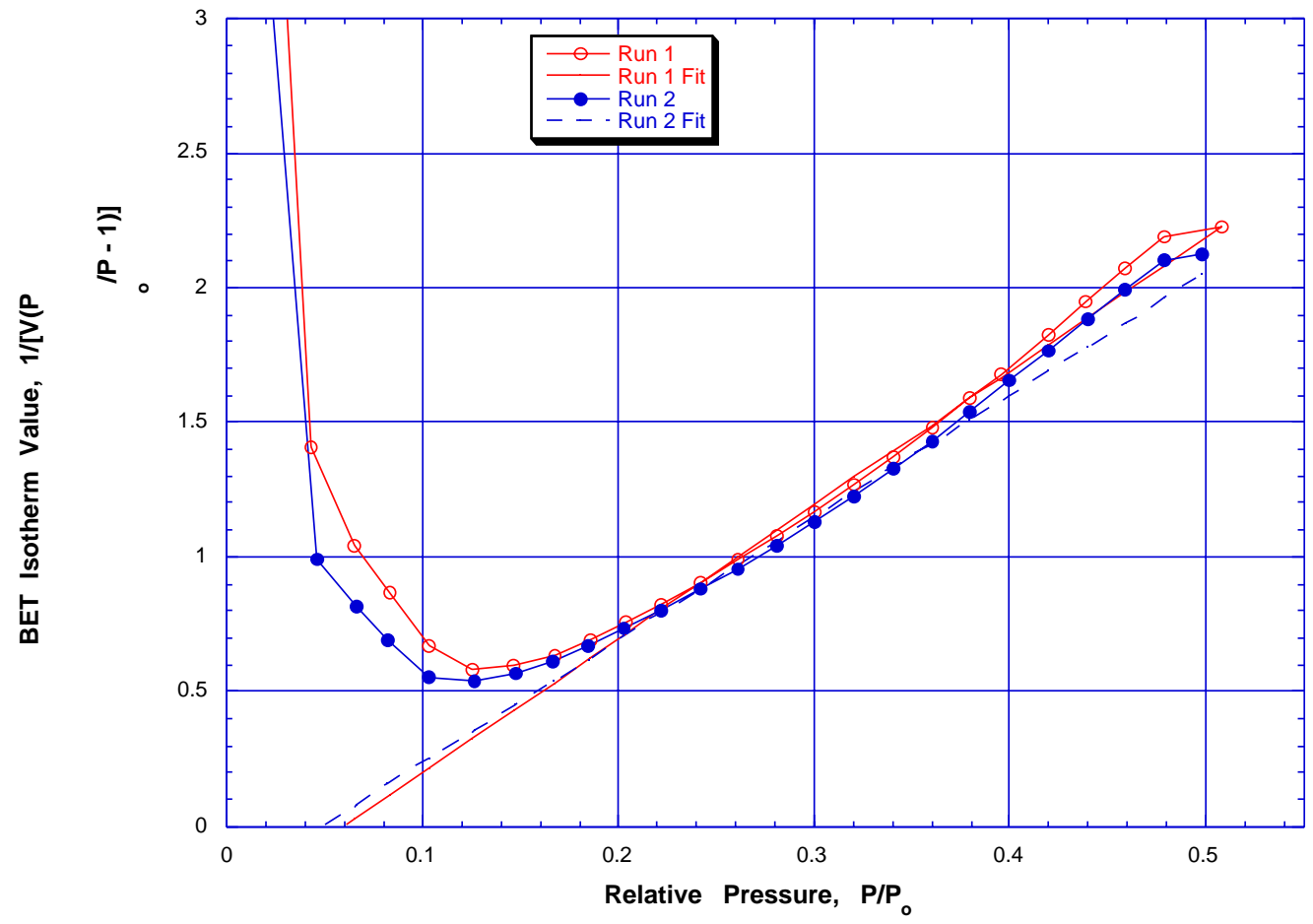

(b) Isotherm

Figure 4.2 Adsorbed Volume (a) and adsorption isotherm (b) for (iii) TSFH16-02, Sample 32. 


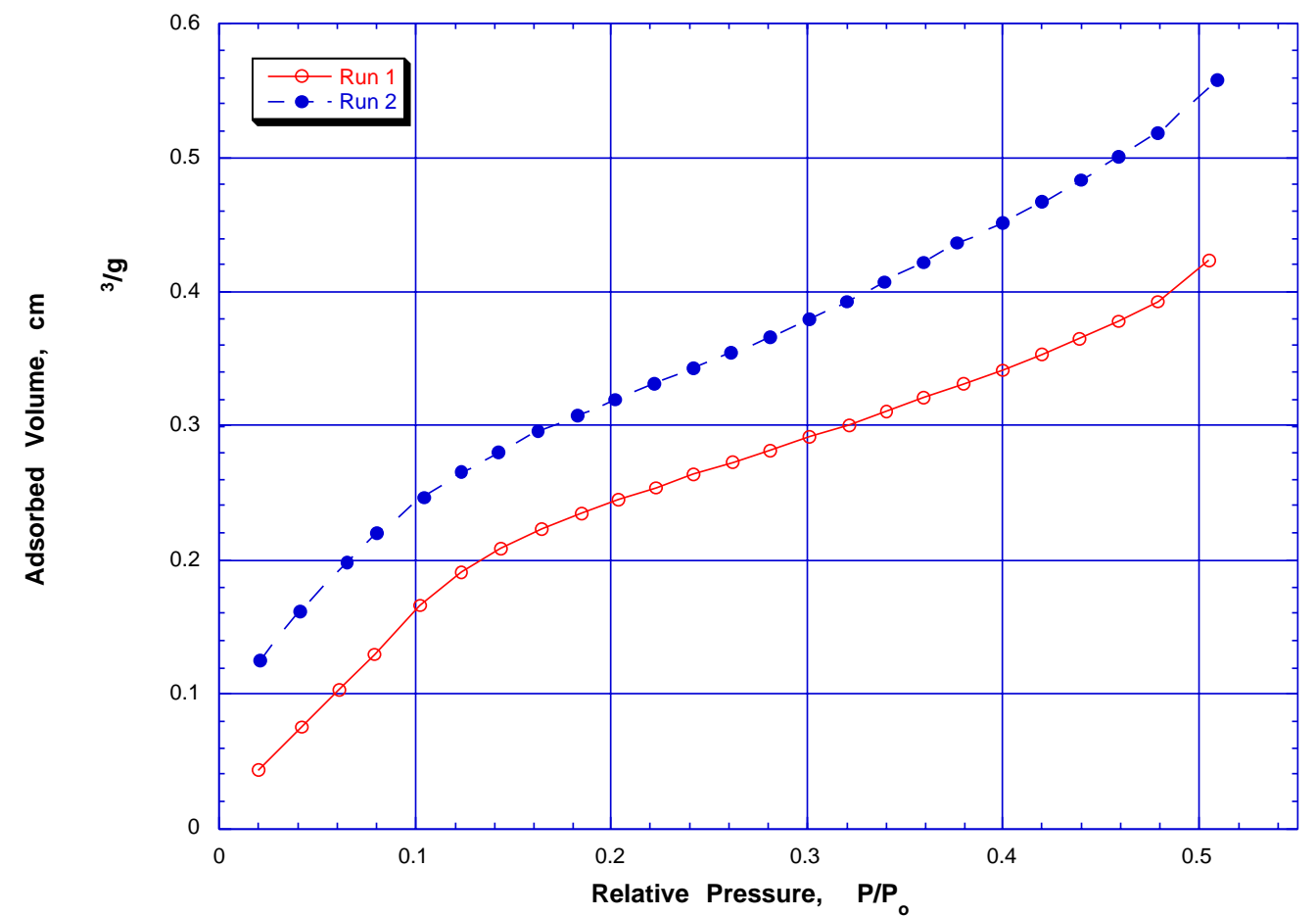

(a) Adsorbed Volume

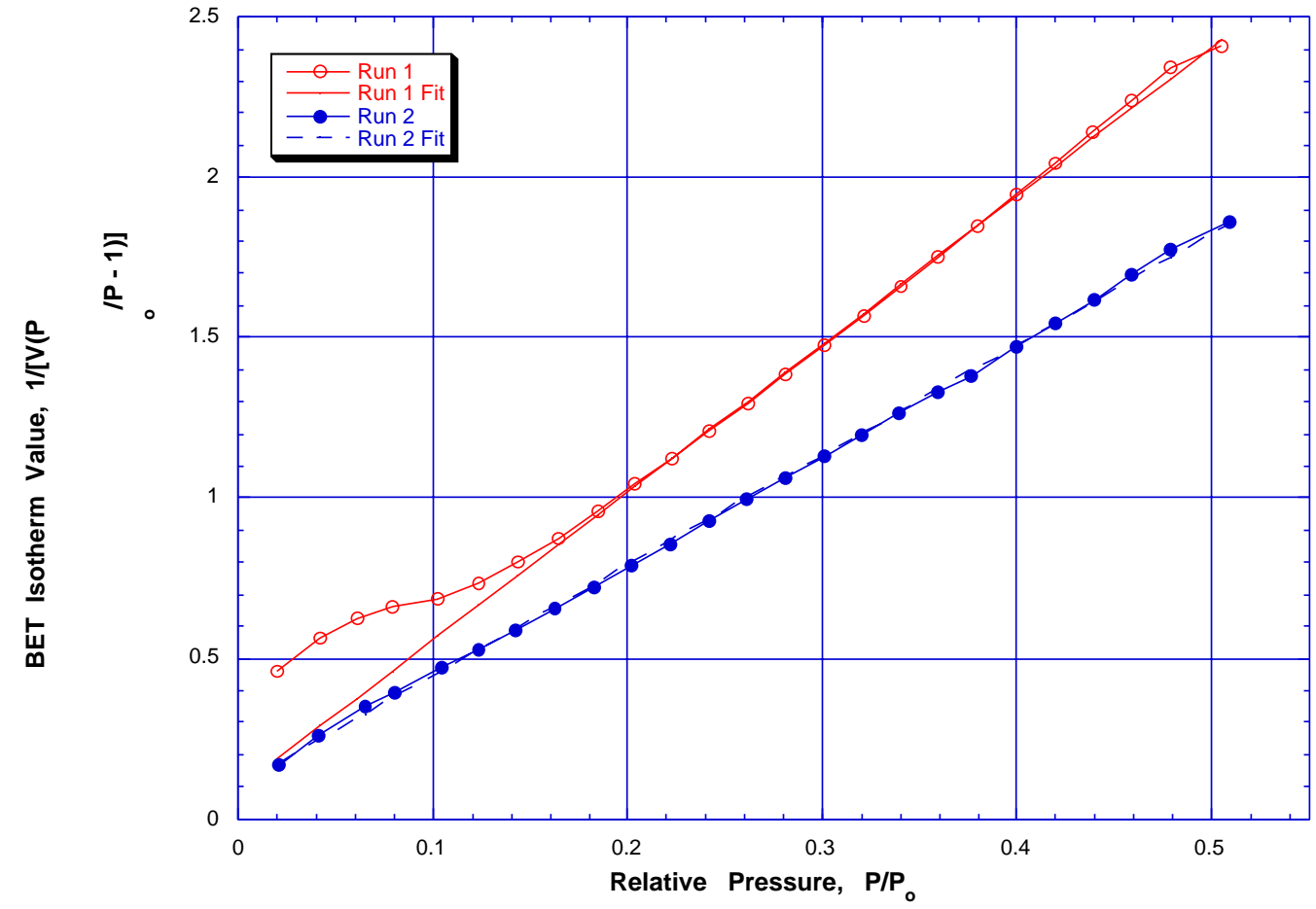

(b) Isotherm

Figure 4.3 Adsorbed Volume (a) and adsorption isotherm (b) for (i) TSFH15-02, Sample 40B. 


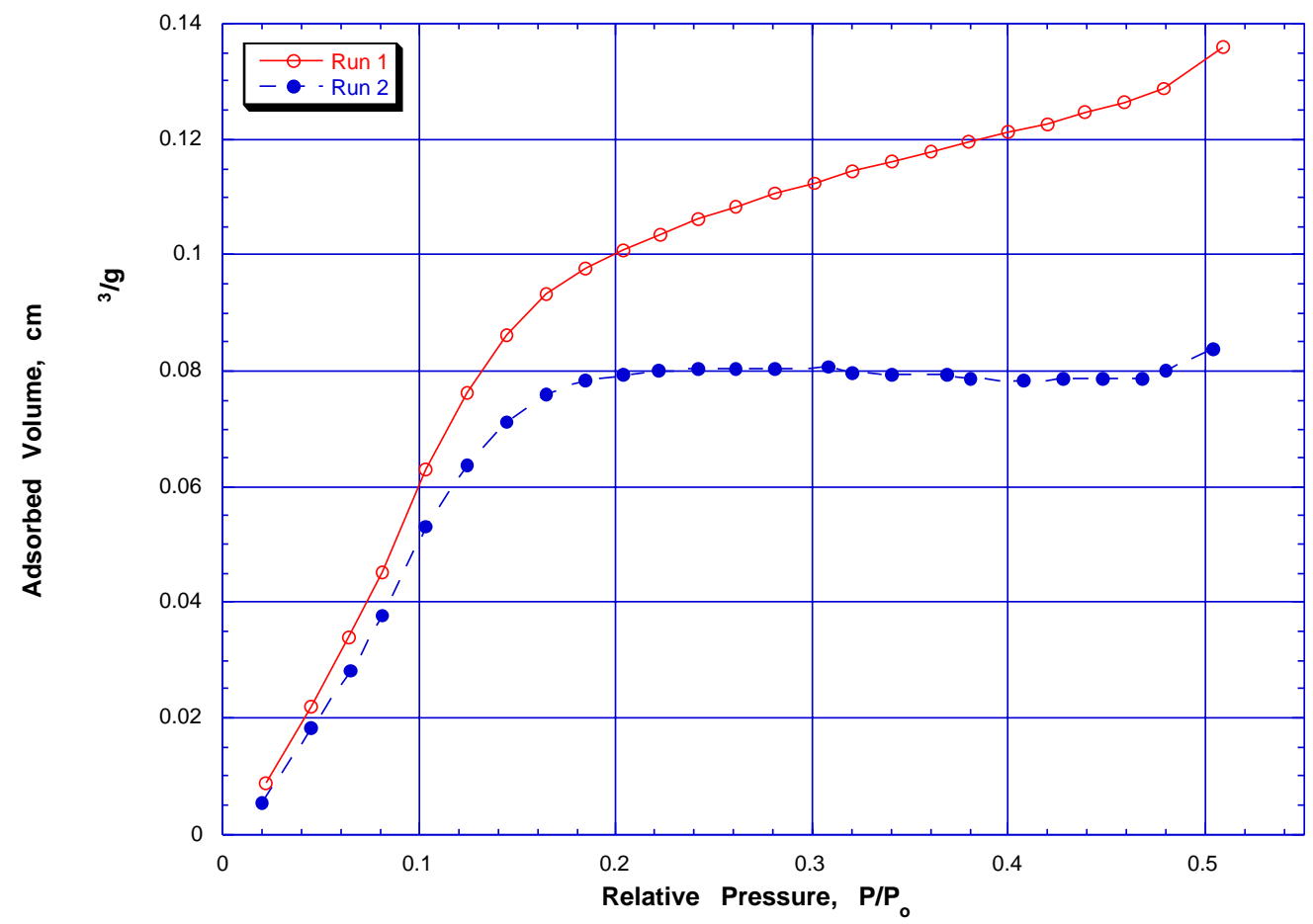

(a) Adsorbed Volume

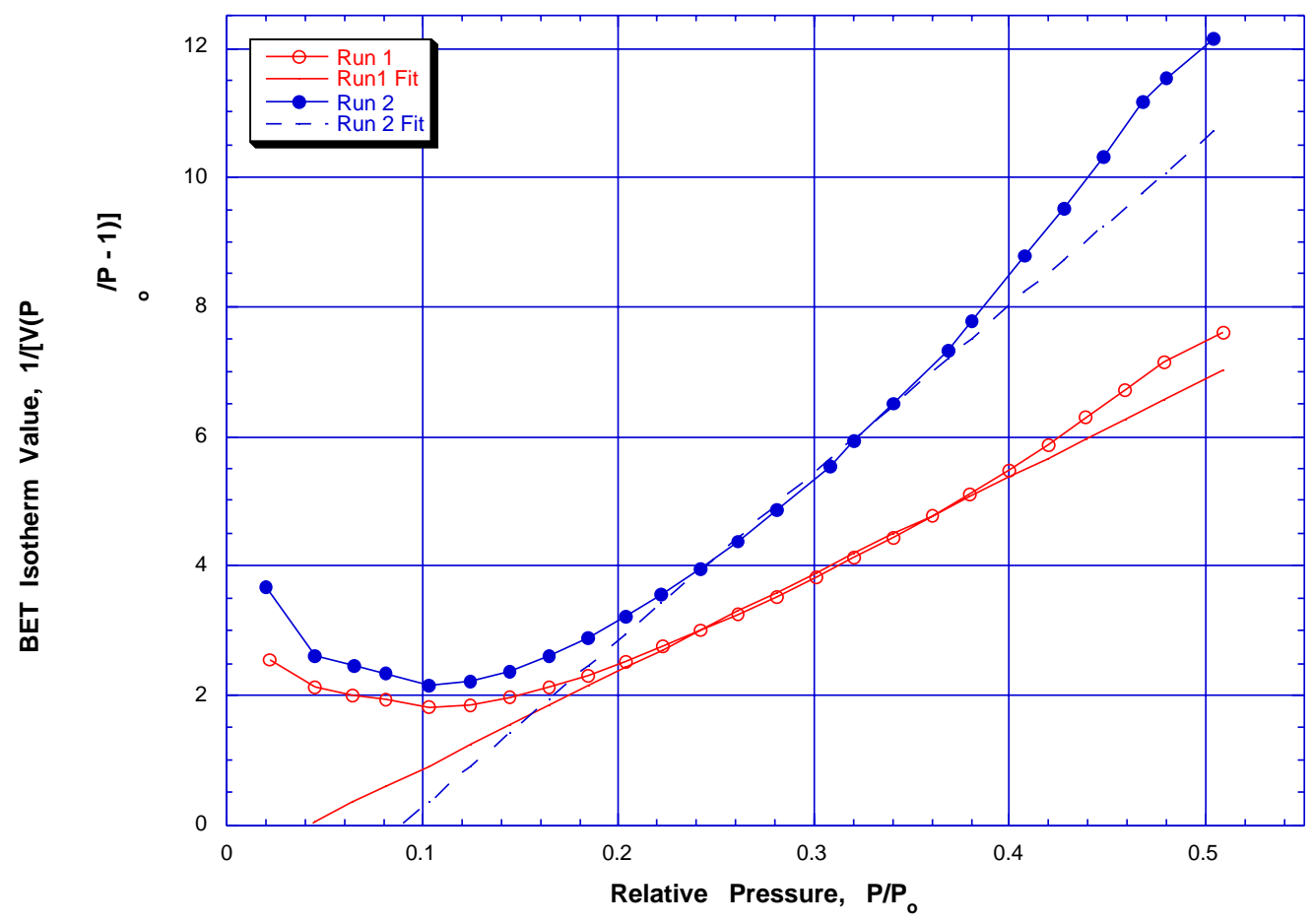

(b) Isotherm

Figure 4.4 Adsorbed Volume (a) and adsorption isotherm (b) for (ii) TSFH19-02, Sample 40A. 


\section{Particulate Composition Analysis by SEM/EDX Analysis}

Qualitative elemental composition analysis of dust collected from Tore Supra was obtained via energy dispersive X-ray analysis. Figure 5.1 shows (a) smaller particulate collected from the upper region of the vessel, and (b) larger particulate collected from the bottom. As indicated in part (a) of the figure, X-ray spot analysis indicated the presence of particles composed of pure carbon, as well as particles containing various quantities of $\mathrm{Fe}, \mathrm{Cr}, \mathrm{Al}, \mathrm{Si}$, and $\mathrm{O}$. The locations identified in part (b) of the figure contain the following:

(1) $\mathrm{Fe}(67.8$ at.\%), $\mathrm{Cr}(18.7$ at.\%), and $\mathrm{Ni}(13.5$ at.\%)

(2) $\mathrm{C}$ (65.0 at.\%), $\mathrm{O}(17.0$ at.\%), $\mathrm{Ca}(9.00$ at\%), and $\mathrm{Cl}(9.00$ at.\%)

(3) $\mathrm{Fe}$ (44.3 at.\%), C (20.0 at.\%), $\mathrm{Cr}$ (14.3 at.\%), Ni (10.0 at.\%), and $\mathrm{O}$ (10.0 at.\%)

(4) $\mathrm{C}$ (72.5 at.\%), $\mathrm{Cl}(14.7$ at.\%), and $\mathrm{O}$ (12.8 at.\%)

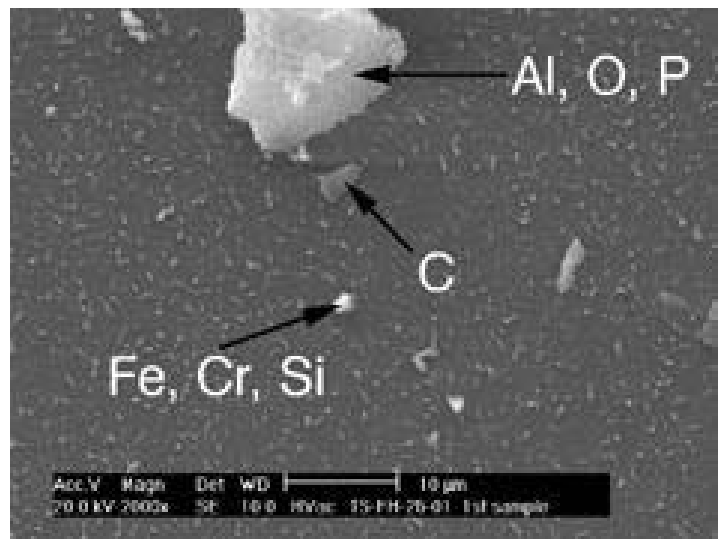

(a) Location 6, Module 1

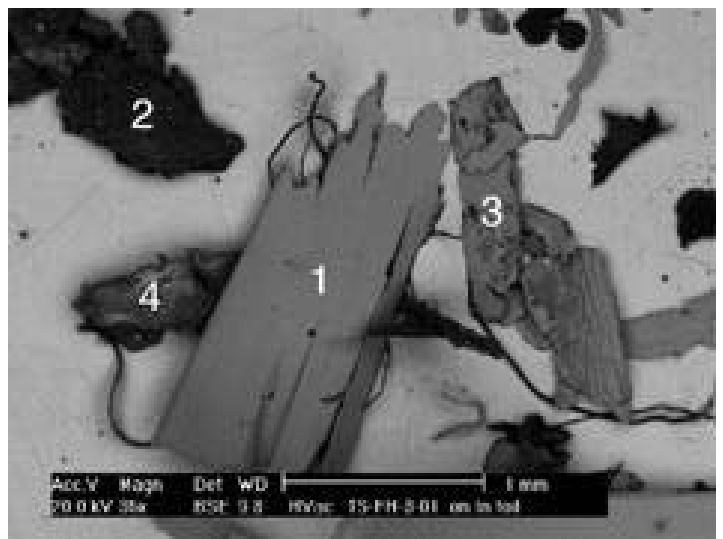

(b) Location 12, Module1

Figure 5.1 SEM images of (a) smaller dust collected from Location 6 and (b) larger debris from Location 12 of Module I.

Quantities of elements such as $\mathrm{Al}, \mathrm{Zn}, \mathrm{Cr}$, and Fe were also found in particles collected from nearly all locations. Figure 5.2 displays the composition of particles with intermediate sizes (5 $10 \mu \mathrm{m})$ collected from Location 6, of Module I (Sample 5).
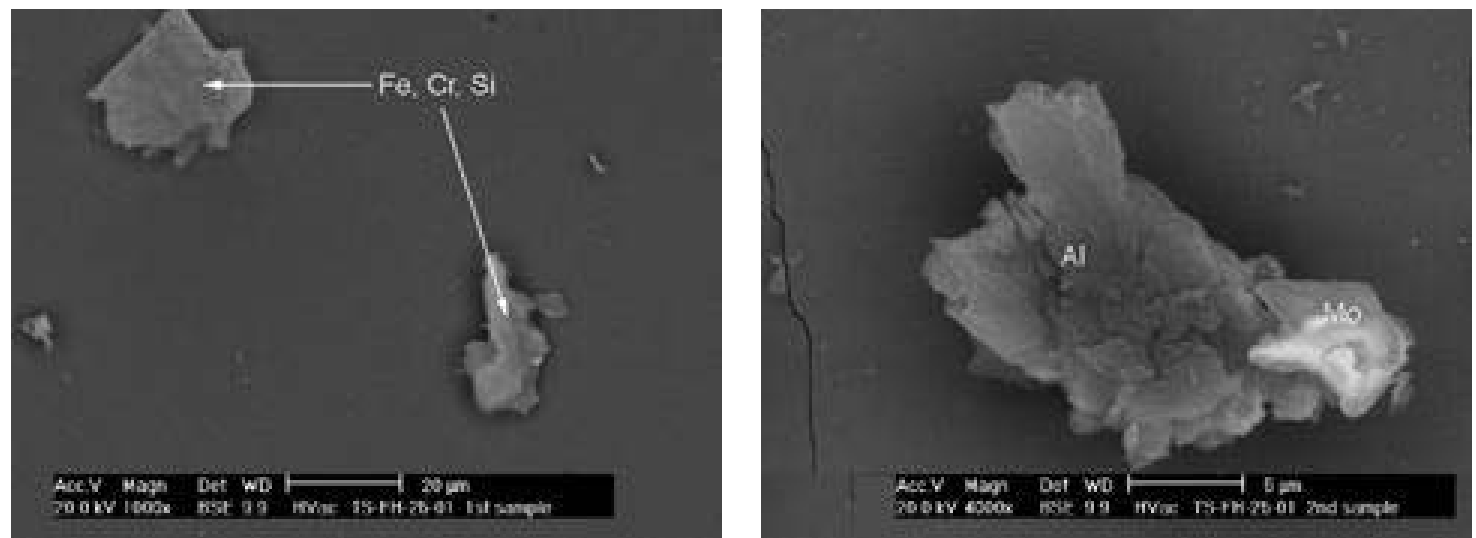

Figure 5.2 Particles from Location 6, of Module I (Sample 5, TSFH25-01). 

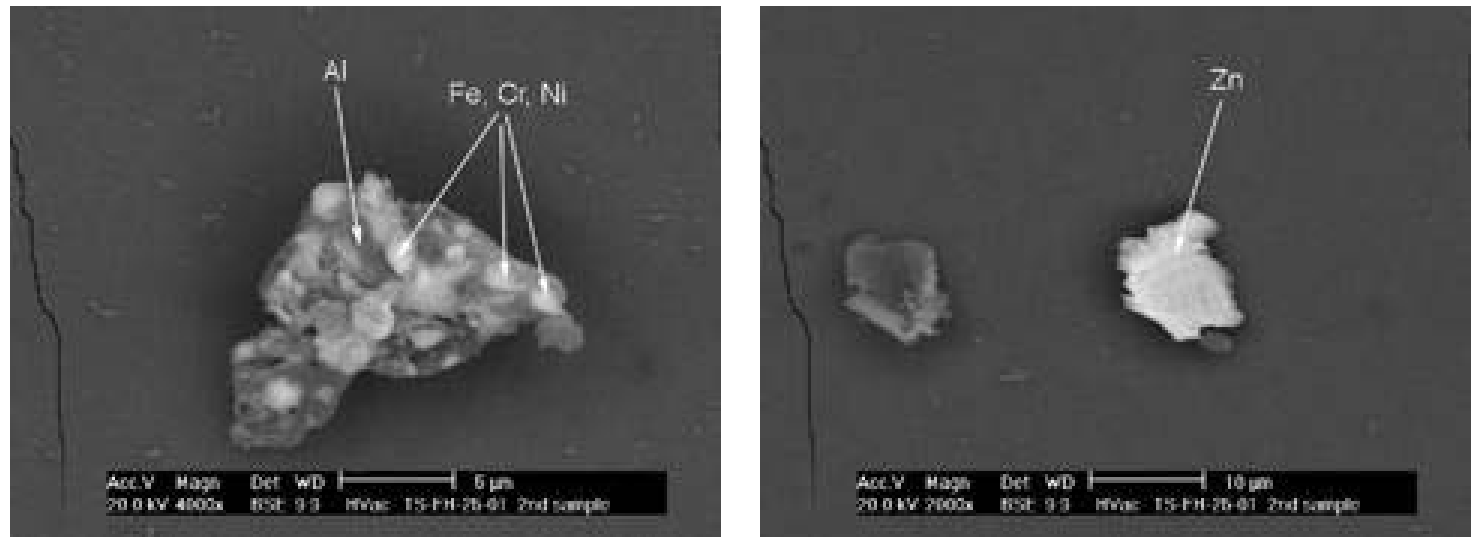

Figure 5.2 continued

\section{Discussion}

\subsection{Distribution of Dust Surface Mass Density}

The total mass of dust collected by each filter is divided by the sampled area to give the surface mass density at each sampling location. Figure 6.1 shows the distribution of surface mass density for all poloidal locations in each toroidal module. With the exception of the sample taken from location 1 in module 1, outboard collection locations have an average surface mass density of $20 \mathrm{mg} / \mathrm{m}^{2}$. The value for location 1 in module 1 is abnormally large $\left(350 \mathrm{mg} / \mathrm{m}^{2}\right)$ because of the presence and collection of melted copper from a lower hybrid heating antenna in the neighboring port. Particulate collected from inboard locations has an average surface mass density of $35 \mathrm{mg} / \mathrm{m}^{2}$, slightly greater than that of the outboard locations. Greater deposition on the inboard first wall tiles is expected because of the local plasma contact during inboard limiter operation. Mass surface density of dust collected from upper surfaces has the lowest value relative to all other poloidal positions, having an average of $5.0 \mathrm{mg} / \mathrm{m}^{2}$. Dust collected at locations 9 and 10 have average surface mass densities of $10.8 \mathrm{mg} / \mathrm{m}^{2}$ and $6.4 \mathrm{mg} / \mathrm{m}^{2}$, respectively. The somewhat larger value that is observed on the stainless steel wing is a result of the proximity of the wing to carbon protective tiles on the lower section of the inner first wall. Each of these surface mass density values contrasts greatly with the values obtained from sampling the vessel floor at locations 11 and 12 . The average value for location 11 is $1800 \mathrm{mg} / \mathrm{m}^{2}$, and for location 12 the value is $560 \mathrm{mg} / \mathrm{m}^{2}$. A significant quantity of the dust's mass inventory therefore resides on the vessel bottom. Of the total dust mass collected in this campaign (440 mg), 76\% (335 mg) was obtained from the lower reaches of the vessel.

An estimation of the total dust mass inventory has been shown ${ }^{4}$ to be $31 \mathrm{~g}$. This estimate was derived by assuming a surface mass distribution of $2000 \mathrm{mg} / \mathrm{m}^{2}$ on $15 \mathrm{~m}^{2}$ of the vessel bottom, and an average of $15 \mathrm{mg} / \mathrm{m}^{2}$ on the remaining $85 \mathrm{~m}^{2}$ of the vessel surface. A different estimation has been performed based on sputtering of plasma facing components. ${ }^{4}$ An integration of all representative plasma discharges for a full experiment campaign (986 discharges) gives $27 \mathrm{~g}$ of eroded carbon. This estimation compares remarkably well with the estimated amount total dust mass in the vessel. 


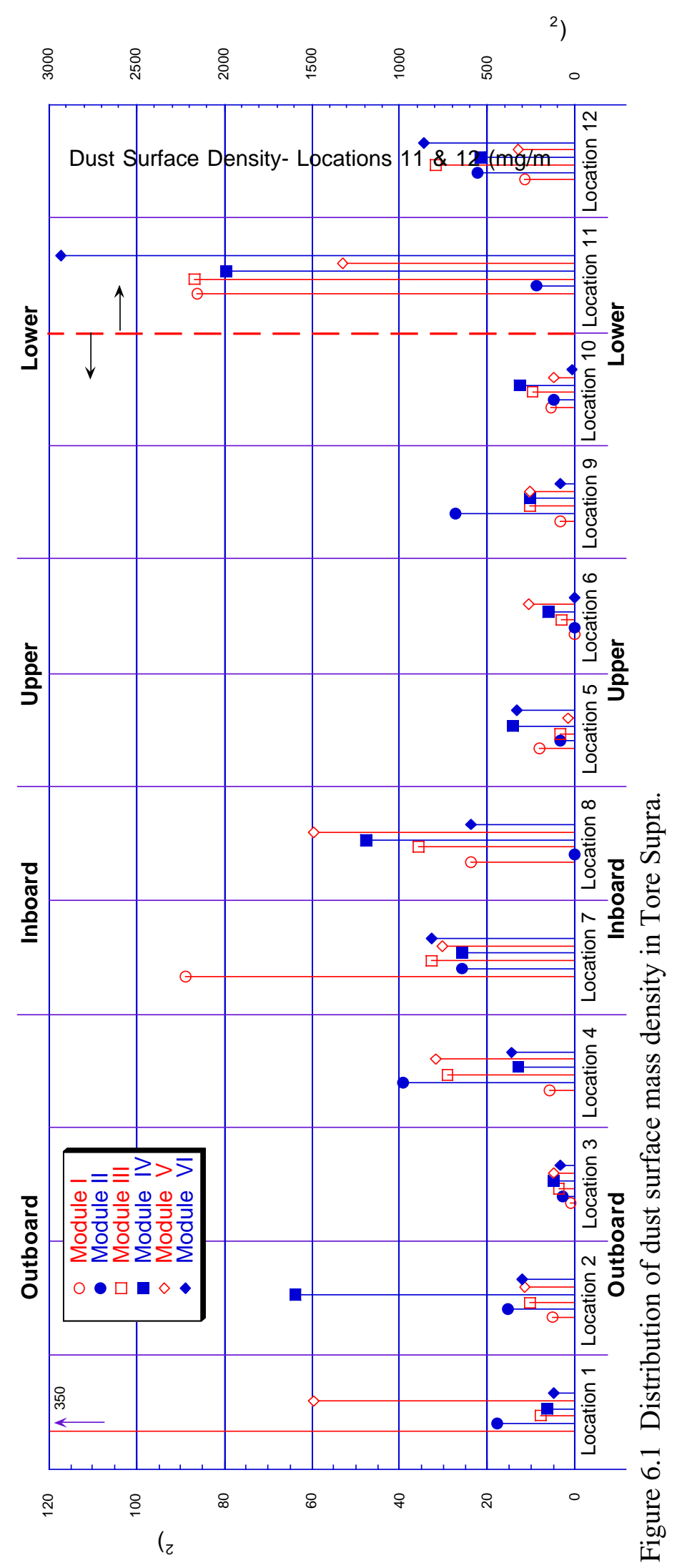

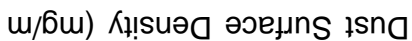




\subsection{Distribution of Dust Size}

Variations of GMD and GSD among all sampled locations are shown in Figure 6.2. Measured GMDs ranged from $0.98 \mu \mathrm{m}$ to $5.3 \mu \mathrm{m}$ with an average value of $3.0 \mu \mathrm{m}$, and the measured GSDs ranged from 1.93 to 4.03. There are no distinguishable position-dependent trends of the data moments at locations other than vessel bottom Locations 11 and 12. These two sample locations were found to have lower than average GMDs and GSDs, even though most of the collected mass came from these positions. Nearly all the mass from these samples is held in the larger particles of the distribution. Smaller particles, however, significantly outnumber the larger ones, and thereby dominate the count-based distribution. This observation has important safety implications because of the difficulty to efficiently control and remove the smaller particulate. 


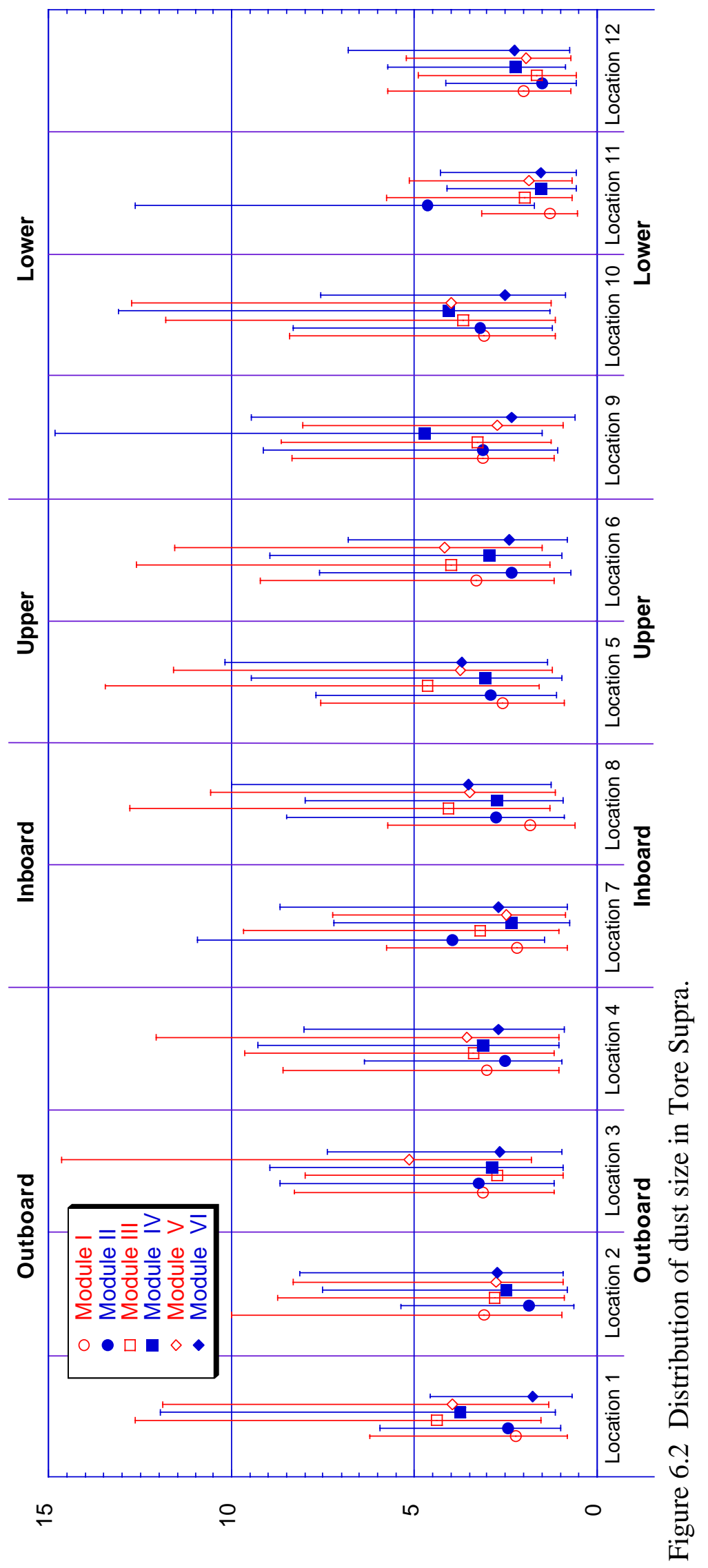

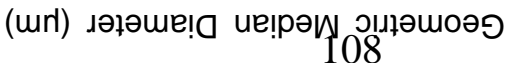




\section{Conclusion}

Tokamak dust collected from various locations within the Tore Supra vessel has been characterized in terms of surface mass density distribution, specific surface area, count-based particle size distribution, and particulate composition. Of the $440 \mathrm{mg}$ of dust collected in this campaign, $76 \%$ was found at locations on the bottom of the vessel. An average of $1100 \mathrm{mg} / \mathrm{m}^{2}$ was collected at these locations, whereas an average of $15 \mathrm{mg} / \mathrm{m}^{2}$ was collected at all other locations. Specific surface area of dust collected from vessel bottom and outboard locations was found to be $1.32 \mathrm{~m}^{2} / \mathrm{g}$. Average particle size from all locations was $3.0 \mu \mathrm{m}$, and the particles were composed of several elements that constitute various components within the vessel. 


\section{References}

1. D. A. Petti, K. A. McCarthy, "Progress in U.S. Fusion Safety and Environmental Activities Over the Last Decade," Fusion Technology, 37, 1-23 (2000).

2. J. Winter, "Dust in Fusion Devices- experimental evidence, possible sources and consequences," Plasma Physics and Controlled Fusion, 40, 1201-1210 (1998).

3. W. J. Carmack, M. E. Engelhardt, P. B. Hembree, K. A. McCarthy, D. A. Petti, "DIII-D Particulate Characterization," INEEL External Report INEEL/EXT-97-00702, November 1997.

4. Ph. Chappuis, J.P. Sharpe, E. Tsitrone, H. Linke, H. Bolt, D.A. Petti, "Dust Characterization and analysis in Tore Supra," to be published in Journal of Nuclear Materials (March 2001).

5. Information on the application of NIHImage are available from the web site: rsb.info.nih.gov/nih-image

6. D.A. McQuarrie, J.D. Simon, Physical Chemistry: A Molecular Approach, University Science Books, Sausalito, CA, 1997.

7. $\quad$ R.A. Anderl, et al., "BET Surface Area Measurements of Materials for Fusion Safety Studies,” ITER Engineering Design File, ITER/US/97/TE/SA-20, January, 1998. 